\title{
Contributions to Automated Realtime Underwater Navigation
}

\author{
by \\ Michael Jordan Stanway \\ S.B., Massachusetts Institute of Technology (2006) \\ S.M., Massachusetts Institute of Technology (2008) \\ Submitted to the Joint Program in Applied Ocean Science and Engineering \\ in partial fulfillment of the requirements for the degree of \\ Doctor of Philosophy \\ at the
MASSACHUSETTS INSTITUTE OF TECHNOLOGY
and the \\ WOODS HOLE OCEANOGRAPHIC INSTITUTION \\ February 2012 \\ (c) Michael Jordan Stanway, MMXII. All rights reserved. \\ The author hereby grants to MIT and WHOI permission to reproduce and publicly \\ distribute paper and electronic copies of this thesis document in whole or in part.
}

Author

Joint Program in Applied Ocean Science and Engineering

December 12, 2011

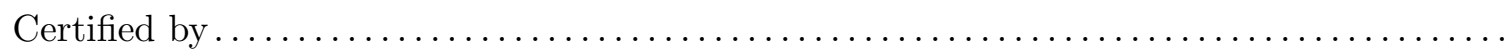

Dana R. Yoerger

Senior Scientist, Woods Hole Oceanographic Institution

Thesis Supervisor

Accepted by

David E. Hardt

Chairman, Department of Mechanical Engineering Committee on Graduate Theses

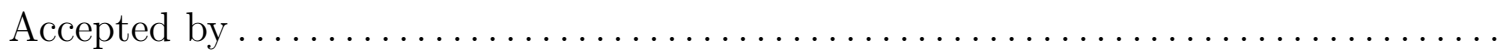

James C. Preisig

Chairman, Joint Committee for Applied Ocean Science and Engineering 


\title{
Contributions to Automated Realtime Underwater Navigation
}

by

\author{
Michael Jordan Stanway
}

\begin{abstract}
Submitted to the Joint Program in Applied Ocean Science and Engineering on December 12, 2011, in partial fulfillment of the requirements for the degree of

Doctor of Philosophy
\end{abstract}

\begin{abstract}
This dissertation presents three separate-but related-contributions to the art of underwater navigation. These methods may be used in postprocessing with a human in the loop, but the overarching goal is to enhance vehicle autonomy, so the emphasis is on automated approaches that can be used in realtime. The three research threads are: i) in situ navigation sensor alignment, ii) dead reckoning through the water column, and iii) model-driven delayed measurement fusion. Contributions to each of these areas have been demonstrated in simulation, with laboratory data, or in the field-some have been demonstrated in all three arenas.

The solution to the in situ navigation sensor alignment problem is an asymptotically stable adaptive identifier formulated using rotors in Geometric Algebra. This identifier is applied to precisely estimate the unknown alignment between a gyrocompass and Doppler velocity log, with the goal of improving realtime dead reckoning navigation. Laboratory and field results show the identifier performs comparably to previously reported methods using rotation matrices, providing an alignment estimate that reduces the position residuals between dead reckoning and an external acoustic positioning system. The Geometric Algebra formulation also encourages a straightforward interpretation of the identifier as a proportional feedback regulator on the observable output error. Future applications of the identifier may include alignment between inertial, visual, and acoustic sensors.

The ability to link the Global Positioning System at the surface to precision dead reckoning near the seafloor might enable new kinds of missions for autonomous underwater vehicles. This research introduces a method for dead reckoning through the water column using water current profile data collected by an onboard acoustic Doppler current profiler. Overlapping relative current profiles provide information to simultaneously estimate the vehicle velocity and local ocean current-the vehicle velocity is then integrated to estimate position. The method is applied to field data using online bin average, weighted least squares, and recursive least squares implementations. This demonstrates an autonomous navigation link between the surface and the seafloor without any dependence on a ship or external acoustic tracking systems.
\end{abstract}


Finally, in many state estimation applications, delayed measurements present an interesting challenge. Underwater navigation is a particularly compelling case because of the relatively long delays inherent in all available position measurements. This research develops a flexible, model-driven approach to delayed measurement fusion in realtime Kalman filters. Using a priori estimates of delayed measurements as augmented states minimizes the computational cost of the delay treatment. Managing the augmented states with time-varying conditional process and measurement models ensures the approach works within the proven Kalman filter framework-without altering the filter structure or requiring any ad-hoc adjustments. The end result is a mathematically principled treatment of the delay that leads to more consistent estimates with lower error and uncertainty. Field results from dead reckoning aided by acoustic positioning systems demonstrate the applicability of this approach to real-world problems in underwater navigation.

Thesis Supervisor: Dana R. Yoerger

Title: Senior Scientist, Woods Hole Oceanographic Institution 


\section{Acknowledgements}

Lots of people have helped me get to this point-earning my Doctorate-I want to thank all of you.

First and foremost, I want to thank Lauren Cooney for keeping me sane... ... more or less.

Dr. Dana Yoerger, my advisor, taught me what it means to be an engineer supporting and driving science in the field, which is exactly what I came to learn from him. My committee, Prof. Alex Techet, Prof. Franz Hover, and Dr. James Kinsey asked thoughtful questions, provided helpful suggestions, and supported me through the entire process.

I'd like to thank the ABE/Sentry group for taking me to sea and letting me work on such interesting things with such cool vehicles. Dana \& James showed me how the brains work, and let me run the prelaunch. Al Bradley always had a long (and instructive, and valuable) answer to a short question. Rod Catanach \& Andy Billings taught me how to handle things on deck and kept the vehicle running smoothly. $\mathrm{Al}$ Duester rebuilt the power system at sea, and let me help by scrubbing circuits-Scotty McCue was always there to help, and demonstrate proper circuit scrubbing technique. Carl Kaiser has been great at organizing us to get things done. In the past few years, I think I got just about the best introduction to field robotics I could have asked for.

I'd also like to thank the robots-ABE and Sentry. I know they aren't sentient, but it's hard not to get attached when you work with them.

I'd like to thank my draft readers and presentation watchers for their patience and helpful comments: Lauren Cooney, Judy Fenwick, Al Bradley, Jon Howland, Mike Jakuba, Giancarlo Troni, Stefano Suman, Peter Kimball, Gregor Cadman, Alex Kessinger, Brooks Reed, John Leonard, Chris Murphy, Clay Kunz, Heather Beem, Wu-Jung Lee, and of course, my committee again.

My friends and fellow JP students have provided advice on code or prose, sounding boards for crazy ideas, or necesssary distractions. I thank you all. Gregor \& Elaine Cadman provide me with a home away from home when I need to be in Cambridge or 
Boston-Alex Donaldson and An Vu do the same. The echolocators-Lauren, Bridget, Heather, Thaddeus (and Mike), Tim, and David-are always there for me. Heather Hornick \& Bella give us a place to stay and friends to visit in Monterey. Annie, Mark, Matt \& Heidi, and Chris help me blow off steam on the Cape. The Starks were great landlords and remain good friends. JP students \& WHOI postdocs make life interesting around here-particularly (but in no particular order): Chris Murphy, Clay Kunz, Jeff Kaeli, Mark van Middlesworth, Peter Kimball, Wu-Jung Lee, Heather Beem, Derya Akkaynak Yellin, Kalina Gospodinova, Vera Pavel \& Jimmy Elsenbeck, Kim Popendorf, Erin Banning, and Skylar Bayer.

Melissa Keane and Judy Fenwick have made my life at DSL easier by handling all sorts of administrative things, and by helping me keep track of seagoing advisors. I'd also like to recognize Julia Westwater, Tricia Morin Gebbie, Michelle McCafferty, Christine Charette, and Valerie Caron at the WHOI Academic Programs Office. Leslie Regan and Joan Kravit handled things wonderfully on the MIT and Mechanical Engineering side.

During my time working on this research, I have been financially supported by: the National Defense Science and Engineering Graduate (NDSEG) Fellowship administered by the American Society for Engineering Education, the Edwin A. Link Foundation Ocean Engineering and Instrumentation Fellowship, and WHOI Academic Programs office.

Finally, I would like to thank everyone in my family for always being supportive helping where they can. My siblings and their families: Aaryn and Mike Curl; Lauren, Ryan, Carson, and Archer Rutland; Wesley Stanway. My parents, Rose and Mark, read several drafts of this dissertation and the papers that led up to it. They also let me spread it out all over their pool table when I was trying to pull it together toward the end.

I'm not quite sure where I am going, but I know I'll keep learning from all of you. 


\section{Contents}

$\begin{array}{ll}\text { Contents } & 7\end{array}$

$\begin{array}{ll}\text { List of Figures } & 11\end{array}$

$\begin{array}{ll}\text { List of Tables } & 15\end{array}$

$\begin{array}{lll}0 & \text { Introduction } & 17\end{array}$

0.1 Motivation . . . . . . . . . . . . . . . . . . . . 18

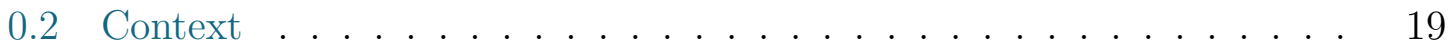

0.2 .1 Acoustic positioning systems . . . . . . . . . . . 20

0.2 .2 Dead Reckoning . . . . . . . . . . . . . . . . . 23

0.2 .3 Inertial Navigation . . . . . . . . . . . . . . . . 27

0.2 .4 Terrain-Relative Navigation $\ldots \ldots \ldots \ldots$

0.2 .5 NDSF AUV Sentry . . . . . . . . . . . . . . . . . . 29

0.3 Thesis Statement . . . . . . . . . . . . . . . . . . 30

0.3 .1 Objectives . . . . . . . . . . . . . . . 31

0.4 Dissertation Structure . . . . . . . . . . . . . . . . . . . 32

1 Navigation Sensor Alignment Using Rotors 35

1.1 Introduction . . . . . . . . . . . . . . . . 35

1.1.1 Motivation. ................... 36

1.1.2 Problem: to identify a rotation from a pair of vectors . . . . 36

1.1.3 Related Work . . . . . . . . . . . . . . . . . . . . . . 38

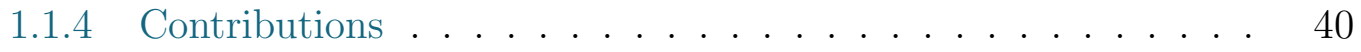


1.2 Geometric Algebra Primer . . . . . . . . . . . . . . . . . . 42

1.2.1 Multiplying Vectors . . . . . . . . . . . . . . . . . . 42

1.2 .2 Rotating vectors . . . . . . . . . . . . . . 45

1.3 Asymptotically Stable Rotor Identifier _ . . . . . . . . . . . . 48

1.3.1 Plant Definition . . . . . . . . . . . . . . . . . . . . 49

1.3 .2 Error Metrics . . . . . . . . . . . . . . . . . . . . . . 49

1.3.3 Proportional Gain Identifier . . . . . . . . . . . . . . 52

1.3.4 Asymptotic Stability . . . . . . . . . . . . . . 55

1.4 Application to Dead Reckoning for Underwater Navigation . . . . . 59

1.4.1 Dead Reckoning and the Identification Plant . . . . . . . . . 61

1.4.2 Renavigation and performance metrics . . . . . . . . . 64

1.5 Laboratory Experiments . . . . . . . . . . . . . . . . . . . . . 69

1.5.1 JHU Hydrodynamics Test Facility and JHUROV . . . . . . 70

1.5.2 Alignment identification and renavigated trajectory . . . . . 71

1.5.3 Cross-validation . . . . . . . . . . . . . . . . . . . 74

1.5.4 A note on transient response . . . . . . . . . . . . . 77

1.6 Field Experiments . . . . . . . . . . . . . . . . . . . . . 80

1.6.1 Sentry field deployments on the Håkon Mosby mud volcano . . 81

1.6.2 Alignment identification and renavigated trajectory . . . . . 82

1.6.3 Cross-validation . . . . . . . . . . . . . . . . . 85

1.7 Discussion and Conclusions . . . . . . . . . . . . . . 87

2 Dead Reckoning Through the Water Column 91

2.1 Introduction . . . . . . . . . . . . . . . . 91

2.1.1 To navigate from the surface to the seafloor and back . . . . 93

2.1.2 Related Work . . . . . . . . . . . . . . . . . . . . 94

2.1 .3 Contributions . . . . . . . . . . . . . . 95

2.2 Concept of Operations . . . . . . . . . . . . . . . 96

2.3 Implementation . . . . . . . . . . . . . . 96

2.3 .1 Online bin-average . . . . . . . . . . . . . . . . 98 
2.3.2 Batch weighted least squares . . . . . . . . . . . . . . . 101

2.3.3 Recursive least squares . . . . . . . . . . . . . . . . . 102

2.4 Field Experiences . . . . . . . . . . . . . . . . . . . . . . . . . . . 104

2.4.1 The AUV Sentry . . . . . . . . . . . . . . . . . 105

2.4.2 Sentry 059: Galápagos Ridge . . . . . . . . . . . . 106

2.4.3 Sentry069: Juan de Fuca . . . . . . . . . . . . . . . . . 110

2.4.4 Sentry085: Kermadec Arch . . . . . . . . . . . . . . . 112

2.5 Discussion and Conclusions . . . . . . . . . . . . . . . . 114

3 Model-Driven Delayed Measurement Fusion $\quad 117$

3.1 Introduction . . . . . . . . . . . . . . . . . . 117

3.1.1 Delayed measurements in underwater positioning . . . . . 118

3.1 .2 Related Work . . . . . . . . . . . . . . . . . . . . 120

3.1 .3 Contributions . . . . . . . . . . . . . . . . . . 124

3.2 Types of measurement delay . . . . . . . . . . . . . . . . 126

3.3 Model-driven delayed measurement fusion . . . . . . . . . . . 127

3.3.1 Timeline and nomenclature for delayed measurements . . . . . 127

3.3.2 The Kalman filter framework _. . . . . . . . . . . . . . . 129

3.3.3 State augmentation . . . . . . . . . . . . . . . . . 132

3.3.4 Time-variant conditional models . . . . . . . . . . . . . . . 133

3.4 A canonical example: the damped harmonic oscillator . . . . . . . . 135

3.4 .1 System Dynamics . . . . . . . . . . . . . . . . . 135

3.4.2 Discrete State Space Model . . . . . . . . . . . . . . . 136

3.4.3 Full measurements . . . . . . . . . . . . . . . . . 136

3.4.4 Fusing measurements with different sampling periods . . . . 137

3.4.5 Fusing delayed measurements without compensation . . . . . . 140

3.4.6 Fusing delayed measurements with compensation . . . . . . . 141

3.4.7 Some comments on alternate approaches . . . . . . . . . 145

3.5 USBL-aided Dead Reckoning in Two Dimensions . . . . . . . . . . 147

3.5 .1 Mission Scenario . . . . . . . . . . . . . . . . . 147 
3.5.2 Delay Compensation . . . . . . . . . . . . . . . . . . . 151

3.5.3 Sentry059: Galápagos Ridge . . . . . . . . . . . . . . 153

3.6 LBL-aided Dead Reckoning in Three Dimensions . . . . . . . . . . 157

3.6 .1 Mission Scenario . . . . . . . . . . . . . . . . . . . . 157

3.6.2 Delay Compensation . . . . . . . . . . . . . . . . . . 162

3.6.3 Sentry073: Håkon Mosby Mud Volcano . . . . . . . . . . . . 166

3.7 Discussion and Conclusions . . . . . . . . . . . . . . . 170

4 Discussion and Conclusions $\quad 173$

$\begin{array}{ll}\text { Bibliography } & 177\end{array}$

$\begin{array}{ll}\text { A Sigma Point Kalman Filters } & 191\end{array}$

A.1 The Unscented Transform . . . . . . . . . . . . . . . . . . . . . 191

A.2 The Unscented Kalman Filter . . . . . . . . . . . . . . . . . . 192 


\section{List of Figures}

0-1 NDSF AUV Sentry and HOV Alvin . . . . . . . . . . . . 19

$0-2$ The NDSF AUV Sentry . . . . . . . . . . . . . . 30

1-1 Rotation between two vectors in 2D and in 3D . . . . . . 37

1-2 Vector products in Geometric Algebra . . . . . . . . . . . . . 43

1-3 Rotor composition methods . . . . . . . . . . . . . . 46

1-4 The components of the estimated rotor $S$ converge to the unknown

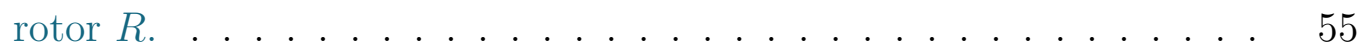

1-5 Evolution of the scalar parameter error when the input/output vector pairs are corrupted by additive white noise. . . . . . . . . 56

1-6 Time evolution of the Lyapunov function $V$ and its derivative $\dot{V}$. . 60

1-7 Phase-space trajectory of the Lyapunov function $V$ for twenty tests with random data. . . . . . . . . . . . . . . . 60

1-8 Evolution of the scalar parameter error, normalized by its initial value. 61

1-9 Components of the position residual from laboratory experiment $070 . \quad 65$

1-10 Distributions of the position residual components from laboratory ex-

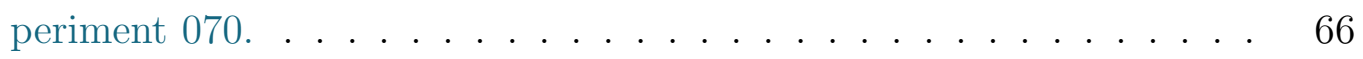

1-11 Distribution of the position residual magnitude from laboratory exper-

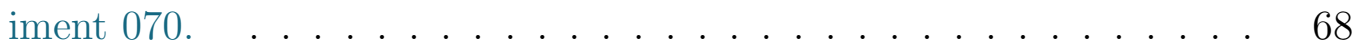

1-12 Cumulative distribution of the position residual magnitude from laboratory experiment $070 . \ldots \ldots \ldots \ldots \ldots \ldots$

1-13 The Johns Hopkins University remotely operated vehicle (ROV) . . 70 
1-14 Residual magnitude distribution or JHUROV laboratory dataset 070, renavigated using different DVL/FOG alignment estimates. . . . .

1-15 Cumulative residual magnitude distribution for JHUROV laboratory dataset 070, renavigated using different DVL/FOG alignment estimates. ....................... . . . . 73

1-16 Self-validation of alignment estimates for each laboratory dataset. . . 76

1-17 Cross-validation of alignment estimates in laboratory experiments. . 78

1-18 Cross-validation statistics of alignment estimates over all laboratory

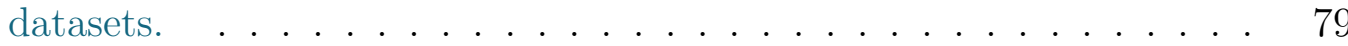

1-19 Scalar residual with respect to alignment estimated by LS-SO(3) method.

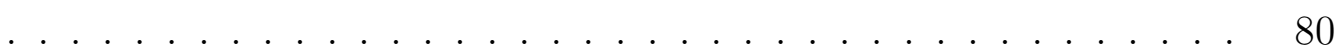

1-20 NDSF AUV Sentry . . . . . . . . . . . . . . . . . . 82

1-21 Distributions of residual components for Sentry075, renavigated using different DVL/FOG alignment estimates. . . . . . . . . . . . 83

1-22 Residual magnitude distribution for Sentry075, renavigated using different DVL/FOG alignment estimates. . . . . . . . . . . . . . . . 84

1-23 Cumulative residual magnitude distribution for Sentry075, renavigated using different DVL/FOG alignment estimates. . . . . . . . . . . . . 84

1-24 Self-validation of alignment estimates for each field dataset. . . . . . 87

1-25 Cross-validation of alignment estimates in field experiments. . . . . . 88

1-26 Cross-validation statistics of alignment estimates over all field datasets. 89

2-1 Surface-to-seafloor navigation using an acoustic Doppler current profiler (ADCP) to measure the current profile. . . . . . . . . . . . . . . . 97

2-2 Current profile and vehicle state estimated by bin-average algorithm

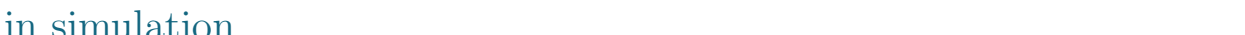

2-3 Error residuals in online bin average simulation results. . . . . . . . 100

2-4 Influence matrix A for a simple system for DR through the water

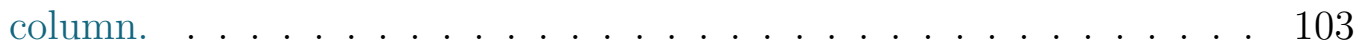


2-5 Echo intensity from water profile measurements during ascent from

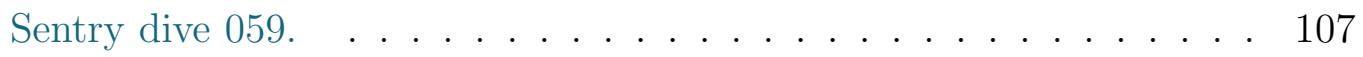

2-6 Correlation magnitude from water profile measurements during ascent from Sentry dive 059. . . . . . . . . . . . . . . . 108

2-7 Error velocity from water profile measurements during ascent from

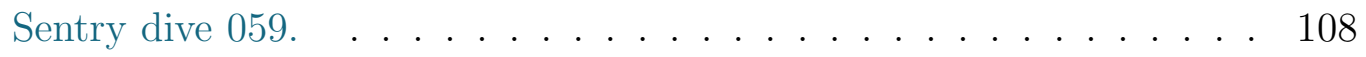

2-8 Estimated ocean current profile and vehicle velocity during ascent from Sentry dive 059. . . . . . . . . . . . . . . . . . . . 109

2-9 Estimated vehicle trajectory during ascent from Sentry dive 059. . . . 110

2-10 Estimated ocean current profile and instrument velocity during ascent from Sentry dive 069. . . . . . . . . . . . . . . 111

2-11 Estimated vehicle trajectory during ascent from Sentry dive 069. . . 112

2-12 Estimated ocean current profile and vehicle velocity during ascent from

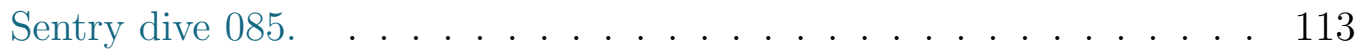

2-13 Estimated vehicle trajectory during ascent from Sentry dive 085. . . . 114

2-14 North and East components of estimated vehicle trajectory during ascent from Sentry dive 085. . . . . . . . . . . . . . . . . 115

3-1 Timeline and nomenclature for delayed measurements. . . . . . . . . 128

3-2 Hidden Markov model network representation of a Kalman filter (KF). 131

3-3 Damped harmonic oscillator simulation with a KF estimating position and velocity and no measurement delay. . . . . . . . . . . . . . 140

3-4 Damped harmonic oscillator simulation with a KF estimating position and velocity and untreated measurement delay. . . . . . . . . . . . . 141

3-5 Damped harmonic oscillator simulation with a KF estimating position and velocity and treated measurement delay. . . . . . . . . . . 145

3-6 Measurement and communication cycle for USBL-aided DR. . . . . 148

3-7 Graph of USBL-aided dead reckoning. . . . . . . . . . . . . . . 151

3-8 Map view of the first survey block on Sentry059. . . . . . . . . . . . 154

3-9 Differences between position estimates on Sentry059. . . . . . . . . 156 
3-10 LBL acoustic positioning using two-way travel times from multiple underwater transponders. . . . . . . . . . . . . . . . . 160

3-11 Measurement cycle for asynchronous, delay-compensated LBL-aided DR with one beacon. . . . . . . . . . . . . . . . 163

3-12 Graph of asynchronous LBL-aided dead reckoning. . . . . . . . . . . . 165

3-13 Map view of vehicle trajectory from Sentry073. . . . . . . . . . . . 167

3-14 Components of vehicle position during Sentry073. . . . . . . . . 168

3-15 Horizontal position differences between asynchronous LBL filters on Sentry073. . . . . . . . . . . . . . . . . . 169

3-16 Horizontal position confidence intervals of asynchronous LBL filters on

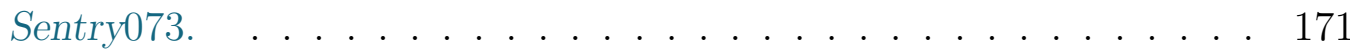




\section{List of Tables}

1.1 Comparison of rotor identifier to related work. . . . . . . . . . . . 41

1.2 Navigation sensors on JHUROV used in laboratory experiments. . . . 71

1.3 Laboratory experiment renavigation residuals . . . . . . . . . . 75

1.4 Navigation sensors on Sentry used in field experiments. . . . . . . . . 81

1.5 Field experiment renavigation residuals . . . . . . . . . . . . 86

2.1 Comparison of DR through the water column to related work. . . . . 95

2.2 Online bin-average water profile navigation error metrics. . . . . . . . 100

3.1 Comparison of model-driven delayed measurement fusion to related work 125

3.2 DHO simulation parameters . . . . . . . . . . . . . . . 139

3.3 Timing, process noise, and measurement noise parameters used in re-

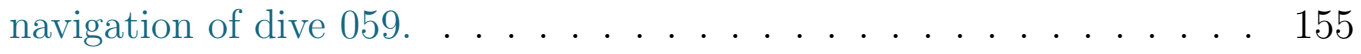




\section{Chapter 0}

\section{Introduction}

66 Billy Cruiser says that the best navigators

are not always certain of where they are,

but they are always aware of their uncertainty.

Frank Bama in Where is Joe Merchant? [1] ,9

This dissertation presents three separate-but related-contributions to the art of underwater navigation. These methods may be used in postprocessing with a human in the loop, but the overarching goal is to enhance vehicle autonomy, therefore the emphasis is on automated approaches that can be used in realtime.

Despite many years of study, underwater navigation remains a rich and unsolved problem. The research presented here draws from fields such as estimation and filtering theory that have strong historical ties to navigation. It also applies techniques from less conventional fields, like Geometric Algebra. The end result is:

- a simple solution to an essential in situ navigation sensor alignment problem,

- a promising approach to dead reckoning through the water column, extending the autonomous navigation capabilities of many underwater vehicles, and

- a straightforward, efficient, and mathematically principled technique for handling measurements that are delayed in time. 
The methods presented here are developed for the challenging task of underwater navigation, but much of this research can and should be applied to problems in other fields.

This chapter first motivates the need for automated realtime underwater navigation, then briefly reviews the background of the problem to provide context. The thesis statement follows, including specific objectives and contributions of this research. The chapter ends with a brief outline of the dissertation structure to provide a roadmap for the reader.

\subsection{Motivation}

Seventy percent of the earth's surface is covered by water. The surface of the ocean is studied by ships, and some of its properties can be measured or estimated using remote sensing techniques. However, the rapid attenuation of electromagnetic waves in water effectively limits most remote sensing to a thin surface layer-there is so much more beneath.

The seafloor is mapped in low resolution using sonars and other sensors from surface ships. This data reveals a system of mid-ocean ridges that circle the globe like the stitching on a baseball. These observations helped the shape the theories of plate tectonics and continental drift. Nowadays, ocean research on the surface, at the seafloor, and in the water column is important for understanding the effects of global warming and pollution.

Underwater vehicles-both manned and unmanned-are critical in our efforts to explore and study the depths of the ocean. Most underwater sensors possess a relatively small field of view; this means they must be carried over large distances to achieve high spatial coverage and resolution. Dynamic changes in the ocean environment also drive a desire to achieve high temporal resolution. Oceanographic submersibles

like Alvin and Sentry (Figure 0-1) collect vast amounts of physical, chemical, and biological data during their missions below the surface. This data is much more valuable when it can be precisely referenced in space and time. This gives rise to the 


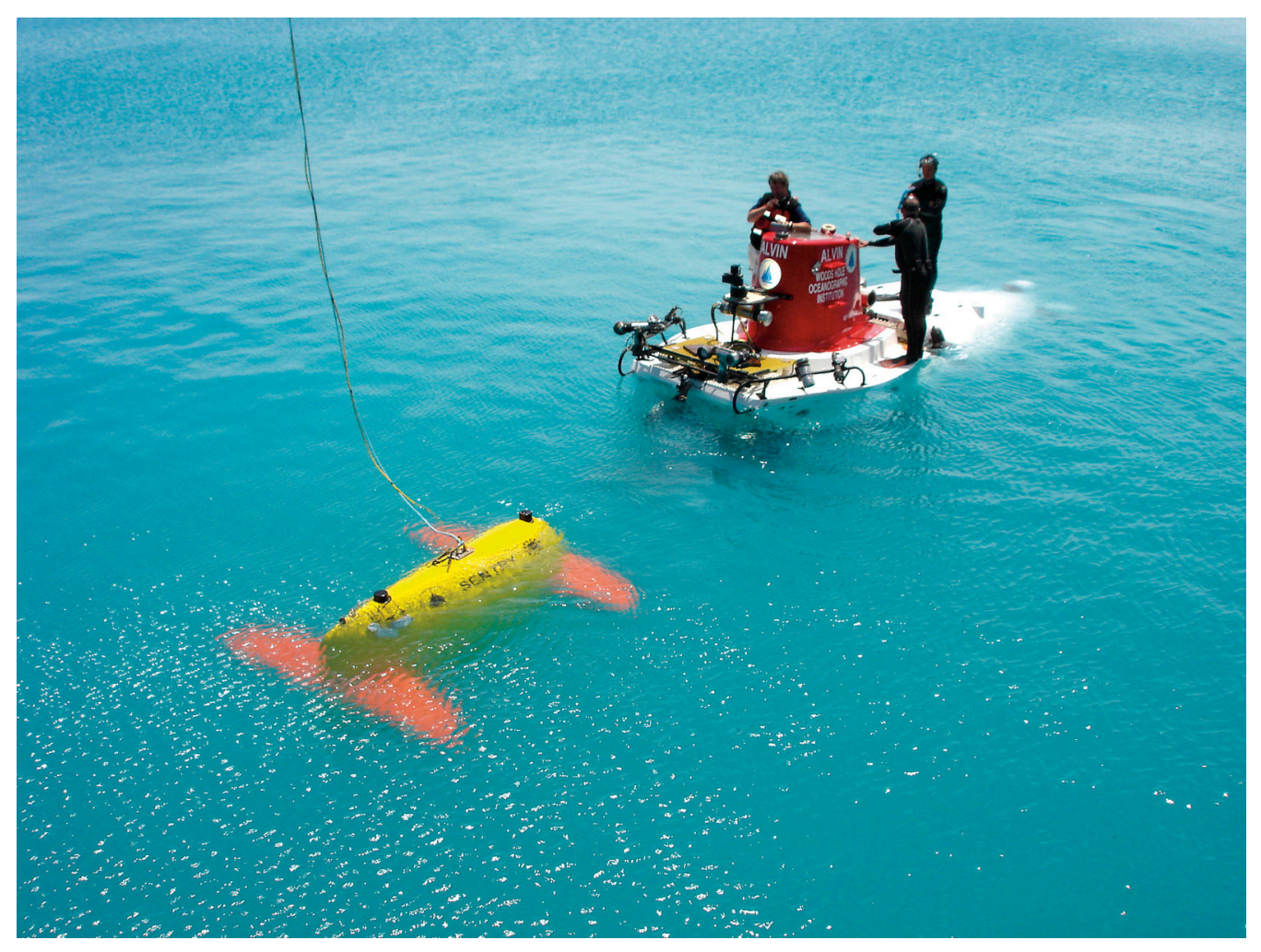

Figure 0-1: The AUV Sentry (left) and the HOV Alvin (right) are oceanographic submersibles operated by the National Deep Submergence Facility (NDSF) at the Woods Hole Oceanographic Institution (WHOI). photo credit: C. German, WHOI

maxim: The data is only as good as the navigation. The key to good navigation is understanding the strengths and limitations of the sensors and algorithms that solve the navigation problem. As we send these vehicles on longer, more difficult missions, we rely on increasing autonomy. This means that each vehicle must navigate its environment in realtime and without intervention from humans or relying on external systems and infrastructure. We need tools and techniques for automated realtime underwater navigation.

\subsection{Context}

This section briefly reviews the background of the underwater navigation problem. It highlights some of the challenges a navigator faces in the underwater environment, and the resources the navigator can rely upon. 
Localization-simply knowing where you are-remains a challenging problem for underwater vehicles. Current state-of-the-art underwater navigation systems fuse measurements from acoustic beacons, a pressure sensor, a Doppler sonar, a Northseeking gyrocompass, and an inertial measurement unit (IMU). Auxiliary sensors like a magnetic compass or an inclinometer are often included as backup. The inertial measurement unit provides acceleration data at a fast update rate, but is subject to inaccuracies from integration error and drift bias over longer timescales. The compass (or gyrocompass) and doppler sonar provide heading and velocity information for dead reckoning. The pressure sensor constrains the estimate of vehicle depth. Long baseline (LBL) or ultra-short baseline (USBL) acoustic tracking systems provide position measurements. These sensors are each subject to different weaknesses in terms of noise, drift, update rate, and range. The key task of the navigator is to intelligently combine information from all available sensors.

\subsubsection{Acoustic positioning systems}

Although the Global Positioning System (GPS) has greatly simplified localization in the air and on the surface of the planet, its electromagnetic signals do not penetrate the oceans. Instead, underwater navigators must rely on other means to determine position. Depth is determined from measurements of pressure, temperature, and conductivity [2]. Long baseline (long baseline (LBL)) acoustic positioning uses travel times to and from multiple transponders to estimate the position of a vehicle relative to those beacons. Ultra-short baseline (USBL) acoustic tracking sytems use travel time and phase from a ship-mounted transducer to compute range, bearing, and azimuth to a submerged transponder. With the growing use of acoustic modems, new techniques using one-way travel times and precision synchronous clocks allow multiple vehicles to serve as navigational beacons for each other.

\section{Long Baseline Acoustic Positioning}


LBL positioning uses ranges from two or more fixed underwater acoustic transponders to localize another transponder, usually on a submerged vehicle or instrument package [3]. The globally referenced transponder positions are surveyed by a ship when the LBL net is deployed. In spherical LBL positioning ${ }^{1}$, the vehicle interrogates the net with an acoustic signal, and each transponder replies in a unique way. Given an estimate of the sound speed through the water, the measured two-way travel times produce estimates of the slant range between the vehicle and each transponder.

Each range imposes a spherical constraint-the vehicle must lie on the sphere with radius equal to the range to that transponder. Two spheres intersect on a circle, three spheres intersect at two points, and four spheres intersect at a single point. In practice, the depths of the transponder and the vehicle are well-known, so the spherical constraints can be collapsed to circular constraints and only three ranges are required. The problem can now be solved by trilateration. The problem can also be solved using a single baseline (two transponders) assuming you know what side of the baseline the vehicle is on. If more ranges are recieved than required, the standard approach is nonlinear least squares.

The standard LBL system for field deployments in the deep ocean has a nominal operating frequency of $12 \mathrm{kHz}^{2}[3,4]$, with range up to $10 \mathrm{~km}$ and precision ${ }^{3}$ of $0.1-10$ $\mathrm{m}[6,7]$. The update rate and measurement latency in this system depend on the range and local sound velocity profile, typically varying from 1 to 20 seconds. Chapter 3 in this dissertation proposes a model-driven approach to deal with this measurement latency in an asynchronous unscented Kalman filter (UKF).

LBL works like an acoustic version of GPS underwater-you just have to provide your own transponders (as opposed to using satellites). The cost is in the ship time spent deploying and surveying the beacons ${ }^{4}$-once that is done, the ship is free to

\footnotetext{
${ }^{1}$ Hyperbolic LBL positioning, on the other hand, uses a passive receiver and synchronized beacons. The receiver position is calculated using the difference in ranges to the two beacons $[4,5]$. We will focus on spherical LBL for the remainder of this section.

${ }^{2}$ As previously mentioned, the vehicle interrogates the net at one frequency, and each transponder replies at a unique frequency-the signals in this system range from $7 \mathrm{kHz}$ to $15 \mathrm{kHz}$, but the nominal operating frequency is $12 \mathrm{kHz}$.

${ }^{3}$ range and geometry-dependent

${ }^{4}$ and recovering them afterward
} 
leave the area for other tasks. An AUV can continue to operate untended without position updates from the ship and without its navigation error growing unbounded. This flexibility makes LBL a good solution when operations will be in the same area for several days.

To avoid the time and cost of deploying and surveying multiple beacons during extended mapping operations, several single-beacon methods have been proposed. These approaches are enabled by improved dead reckoning (DR) capabilities of underwater vehicles. They trade the spatial separation of ranges to multiple transponders at one time in traditional LBL for temporal separation of multiple ranges to a single transponder. In synthetic long baseline (SLBL), an error-state extended Kalman filter $(\mathrm{EKF})$ provides corrections to the DR navigation system based on ranges to a single transponder measured from different positions at different times [8]. By plotting multiple ranges from one beacon along the dead reckoned vehicle trajectory, virtual long baseline (VLBL) enables the computation of a 'running fix' of the vehicle position [9]. With tight integration to an inertial navigation system (INS), the underwater transponder positioning (UTP) principle $[10,11]$ also leverages the changing pose of the vehicle relative to the transponder to estimate vehicle position. In each of these methods, the geographic position of the transponder must still be surveyed.

\section{Ultra-Short Baseline Acoustic Tracking}

When a surface ship is in the area, it can track an underwater vehicle using an ultrashort baseline (USBL) system (e.g., [10,12]). A ship-mounted transducer acoustically interrogates a transponder mounted on the vehicle; the multi-element transducer measures the travel time and the phase of the reply, enabling the system to calculate range, bearing, and azimuth to the transponder. This relative position is then combined with ship position from GPS and orientation from a gyrocompass to compute a geographic position fix for the submerged transponder. If the vehicle requires realtime position measurements, the computed geographic fix can be downlinked via tether (in the case of remotely operated vehicles (ROVs)) or acoustic modem (for manned submersibles or AUVs). 
This mode of operations is attractive because it does not require time to deploy and recover a transponder net-the entire infrastructure consists of a single hull-mounted transducer that moves with the ship. However, precision navigation requires careful calibration of alignment, lateral offsets, and timing between the GPS, gyrocompass, and USBL transducer. Since some ships and systems will move the transducer between operations ${ }^{5}$, this calibration may need to be done at each new site.

One potential drawback of USBL is that the ship must remain in the operating area to track the vehicle. This is not a concern for typical ROV operations, where the vehicle is tethered to the ship anyway, or in many HOV operations, where the ship remains in the area for safety reasons. It is, however, a fundamental limitation for operations with AUVs-once the vehicle is on its mission, the ship could be free to perform other tasks if it did not have to stay in the area to tend to the AUV.

Again, the finite speed of sound in water ensures that any USBL measurements will come with a finite delay. Depending on the water depth, sound velocity profile, and logistical constraints, it will be on the order of $1-10$ seconds between the time a position fix is valid and the time it is available to the vehicle. USBL-aided DR is used as an example for the model-driven delayed measurement fusion approach proposed in Chapter 3 of this dissertation.

\subsubsection{Dead Reckoning}

In its most basic form, dead reckoning (DR) computes a new position estimate by integrating vehicle course and speed over the elapsed time, and adding that change in position to the previous position estimate. This is essentially integrating the firstorder kinematic equations in time. In order to perform DR, the navigator needs measurements or estimates of the vehicle's:

- previous position,

- orientation (just heading in two dimensions), and

\footnotetext{
${ }^{5}$ This is especially true when the USBL transducer is mounted on a retractable mast under the keel or off the side of the ship. This is often done in practice to achieve better acoustic performance below the sea surface and further away from ship noise.
} 
- velocity (vector in two or three dimensions).

The navigator may use the scalar speed and vector course made good in an equivalent formulation. The orientation measurements typically come from a gyrocompass, an attitude and heading reference system (AHRS), or a magnetic compass (e.g., fluxgate). The velocity is often measured using a Doppler velocity log (DVL), but may also be measured by alternate means (such as a pitot tube), or estimated using a dynamic model and measurements of propeller torque and/or shaft speed. The DVL is preferred for velocity measurement because it measures the three components of velocity relative to the seafloor (assumed to be stationary) rather than the speed relative to the water (which may be moving).

DR navigation systems can run at high rates (updating at $1 \mathrm{~Hz}$ or faster) so they enable closed-loop control. The required quantities can all be measured by sensors internal to the vehicle, so DR does not require any external infrastructure. This is advantageous for autonomous and robust operations. However, there are some disadvantages. All forms of DR suffer from integration drift-the errors and uncertainties in velocity and orientation accumulate in the position estimate. This means that the errors and uncertainties in the position estimate grow unbounded in time. For precise navigation, DR systems must be periodically reset or aided with position fixes.

\section{Compass}

The purpose of a compass is to tell the navigator which direction is North-this defines the vehicle attitude relative to a known reference. A magnetic compass orients itself relative to the Earth's magnetic field; a North-seeking gyrocompass aligns itself with the Earth's rotation. Both sensors have different strengths and weaknesses.

Digital magnetic compasses and AHRS are small, rugged, inexpensive $(<\$ 100-$ $\$ 2000)$, and have low power consumption $(<1 \mathrm{~W})$. These sensors use fluxgate or magneto-inductive magnetometers to provide moderately accurate measurements of heading $\left(0.3^{\circ}-3^{\circ}\right)$, pitch, and roll $\left(0.2^{\circ}-2^{\circ}\right)$. Although these sensors are common on underwater vehicles, many factors can hurt their accuracy [6, 7]: 
- magnetic signature of the vehicle structure and systems; this can be partially mitigated by hard- and soft-iron corrections in calibrating the compass, but such calibration does not account for dynamic effects, such as turning on and off motors and other subsystems,

- local magnetic anomalies (e.g., buried cables and pipelines or mineral deposits along mid-ocean ridges or near hydrothermal venting sites),

- alignment with respect to the vehicle reference frame; this must be well-calibrated either in the laboratory or using an in-situ technique such as those presented in $[13,14]$ or $[15]$ and chapter 1 of this dissertation.

Despite these shortcomings, magnetic compasses remain standard equipment on most oceanographic systems due to their small size, low power consumption, and relatively inexpensive cost. Some underwater vehicles (e.g., Jason, Sentry, Nereus) use a gyrocompass as the primary reference, but retain a magnetic compass as backup.

North-seeking gyrocompasses use the earth's rotation to find true North-they do not need to correct for magnetic declination. They are also unaffected by magnetic fields caused by the vehicle and surrounding environment. These factors, along with the superior dynamic heading accuracy $\left(0.1^{\circ}\right.$ or better $)$ make them ideal for highprecision applications [7]. Although mechanical gyrocompasses have been used on surface vessels since the early 1900s, their size, power requirements, and cost prevent their use on small underwater vehicles. Optical gyroscopes measure the interference pattern of two laser beams traveling in opposite directions around a ring. This interference pattern changes based on the rotation rate and the properties of the ring according to the Sagnac effect. Three or more of these optical gyros can be mounted together to observe the three-dimensional rotation rate-this forms an optical gyrocompass. Both ring-laser gyroscope (RLG) and fiber-optic gyroscope (FOG) operate under these principles with slight differences in implementation. They offer increased performance over magnetic compasses, but at the expense of increased size, cost $(>\$ 10,000)$, and power consumption $(\sim 10 \mathrm{~W})$. These systems still need to be carefully aligned with other sensors (especially the DVL), either in laboratory condi- 
tions for integrated units (e.g., [16]) or using in-situ methods in applications where the sensors are independently housed and mounted some distance from each other (e.g., [13-15] and chapter 1 of this dissertation).

\section{Doppler Velocity Log}

A Doppler velocity log (DVL) provides accurate measurements of three-component vehicle velocity over ground within bottom lock range. This range varies with DVL frequency; it is about 250 meters for a $300 \mathrm{kHz}$ DVL [17].

Bottom-lock DVLs have become standard equipment on most oceanographic underwater vehicles. They are used in DR $[18,19]$, in combined LBL/DR navigation systems [20,21], or as an aiding input to an INS [10,22]. In some cases, upward-looking DVLs have been used to measure vehicle velocity relative to overhead ice [23].

The instrument velocity is calculated from the frequency shift in an acoustic signal sent from the DVL to the seafloor, and reflected back to the DVL. A DVL has multiple transducers oriented at different angles so that it can measure the frequency shift along different acoustic beams, then combine the measurements to provide the three-component velocity measurement.

For precision navigation, the alignment of the DVL beams within the instrument is critical. Brokloff describes a matrix algorithm for Doppler navigation in [18]. This algorithm is an improvement on the standard Janus equations which were previously used in marine vehicle navigation. It provides a rigorous mathematical treatment of the different reference frames used in Doppler navigation, and also allows for corrections in transducer-instrument alignment. This algorithm reduced the mean position error in a navigated mission from 0.51 to 0.33 percent distance traveled, and reduced the standard deviation from 0.26 to 0.23 . These errors may seem small, but they are important in precision underwater navigation, as error accumulates over the course of a several kilometer mission.

The hardware and operating principles for a DVL and an acoustic Doppler current profiler (ADCP) are the same-comparing the Doppler shift of an acoustic signal along four different beams gives the velocity of an acoustic scatterer relative to the 
instrument (or vice versa). For a DVL in bottom-lock, the scatterer is the seafloor; for an ADCP, the scatterers are particles suspended in the water column. The same instrument can measure the relative velocity of the bottom and the ocean current.

This has led to some adaptations of DVL-based DR when the vehicle is out of bottom lock range. An accurate measurement of through-the-water vehicle velocity can be made by averaging ADCP measurements over several depth cells. When an ADCP makes concurrent water-profile and bottom-track measurements, the effect of local currents can be estimated. Then, when bottom lock is lost, high-quality velocity estimates can be maintained for a short term [24]. This is often termed 'water track' and the water velocity is measured and averaged over a 'water reference layer' [17]. More recently, researchers have begun to use data from relative water current profiles (i.e., velocity measurements in many discrete range bins) to simultaneously estimate vehicle velocity and ocean current. This can be considered an extension of the Lowered Acoustic Doppler Current Profiler (LADCP) concept used in physical oceanography [25-27]. The problem has been studied using an online bin-average approach [28, 29], an IMU-coupled information filter (IF) implementation [30, 31], weighted least squares (WLS), and recursive least squares (RLS) techniques [29]. This is also the topic of chapter 2 in this dissertation.

\subsubsection{Inertial Navigation}

An IMU contains sensors that measure acceleration and rotation rates in a strapdown package. These sensors are combined with a computer in an INS, so that the measurements can be integrated to provide an inertial pose (position and orientation) estimate from an entirely internal system.

An INS is essentially a second-order DR navigation system-it is subject to the same integration drift and unbounded growth in error and uncertainty as any other DR navigation system. For this reason, an INS in an underwater vehicle is usually aided by measurements from a gyrocompass or AHRS, a DVL, and some combination of acoustic positioning, terrain relative navigation, or visually aided navigation. These external measurements are fused with the inertial estimate to limit the growth of error 
and uncertainty in the estimate.

\subsubsection{Terrain-Relative Navigation}

Given a map of the surrounding environment, a vehicle can localize itself by matching observations from its sensors to areas on the map. Terrain relative navigation (TRN) was originally developed for cruise missile navigation [32,33] but has also been applied to underwater navigation problems. One advantage of terrain relative navigation $(\mathrm{TRN})$ is that there is no infrastructure requirement-it does not rely on systems external to the vehicle. However, it does require a map.

In some situations, an a priori map is available, (e.g., [34,35]). It is more common that an a priori map is not available-either the mission is to explore a new area, or to measure and characterize changes in the terrain. In this case, the vehicle might generate incremental maps from sensor data on the fly (e.g., $[10,36])$ or use simultaneous localization and mapping (SLAM) techniques (e.g., [37-39]).

The terrain may be characterized by sonar range measurements to the seafloor $[10$, $32,33,36,38-41]$ or photographs of the seafloor [42-45]. For some missions, AUVs have navigated relative to sonar maps of moving targets like icebergs [46,47]. The reference for TRN may not even be a conventional map-one of the earlier underwater applications was for submarine navigation relative to the gravitational field of the Earth [35]. Recent work has begun to characterize the performance of TRN using less costly sensors with lower quality measurements-this has the potential to be an enabling technology for inexpensive AUVs that still require long-term autonomous navigation capabilities [32,33].

All TRN techniques are limited by the range of the sensors that measure the terrain. This is typically $\mathcal{O}(10-100 \mathrm{~m})$ for sonar and $<10 \mathrm{~m}$ for photography ${ }^{6}$. In cases without an a priori map, TRN will produce a self-consistent vehicle trajectory and map for the mission, but there will still be uncertainty in the geographic location unless there is another external position measurement ${ }^{7}$.

\footnotetext{
${ }^{6}$ Primarily limited by attenuation of light in water.

${ }^{7}$ e.g., LBL, USBL, or GPS at the surface while the seafloor is within range of the terrain sensor
} 


\subsubsection{NDSF AUV Sentry}

Most of the data used in this dissertation was collected by the AUV Sentry during oceanographic field deployments. The navigational data in Chapter 1 and Chapter 3 is standard and collected during every dive the vehicle makes. The ADCP was reconfigured during several ascents at the end of science missions to collect the relative water current profiles for Chapter 2. This section gives an introduction to Sentry and its systems-sections within each chapter will review the details on the sensors and systems specific to the problem studied in that chapter.

Sentry is a deep submergence AUV designed by the WHOI Deep Submergence Laboratory (DSL) and operated as part of the NDSF. It is the successor to the autonomous benthic explorer (ABE) $[48,49]$, with many design improvements to address the needs of the oceanographic community. Two pairs of control fins with thrusters enable close bottom-following and control at zero speed. The large separation between the center of buoyancy and center of gravity makes Sentry very stable in pitch and roll-this is desirable for high resolution mapping with multibeam or camera. The outer hull is streamlined in the forward direction for efficiency during long surveys, and streamlined in the vertical direction for fast descent and ascent at the beginning and end of dives up to $6500 \mathrm{~m}$ in depth. Science missions often include high-resolution bathymetry, seafloor photography, geomagnetics, and chemical sensing, or some combination. When equipped with a redox potential (eH) probe [50], Sentry is an effective tool for hydrothermal prospecting. More recently, Sentry has used an in-situ mass spectrometer [51] to track and characterize hydrocarbon plumes [52], including the submerged plume from the blowout of the Deepwater Horizon's Macondo well [53].

Sentry's navigation sensor suite includes: an INS with fiber-optic gyroscope (IXSEA PHINS), a $300 \mathrm{kHz}$ DVL/ADCP (Teledyne RD Instruments Workhorse Navigator), a pressure depth transducer (Paroscientific Digiquartz), an LBL positioning system (WHOI), a USBL tracking system with an integrated acoustic modem (Sonardyne), and a backup magnetic compass (PNI). Since the FOG and DVL provide highaccuracy inputs, the standard realtime navigation is dead reckoning without external 


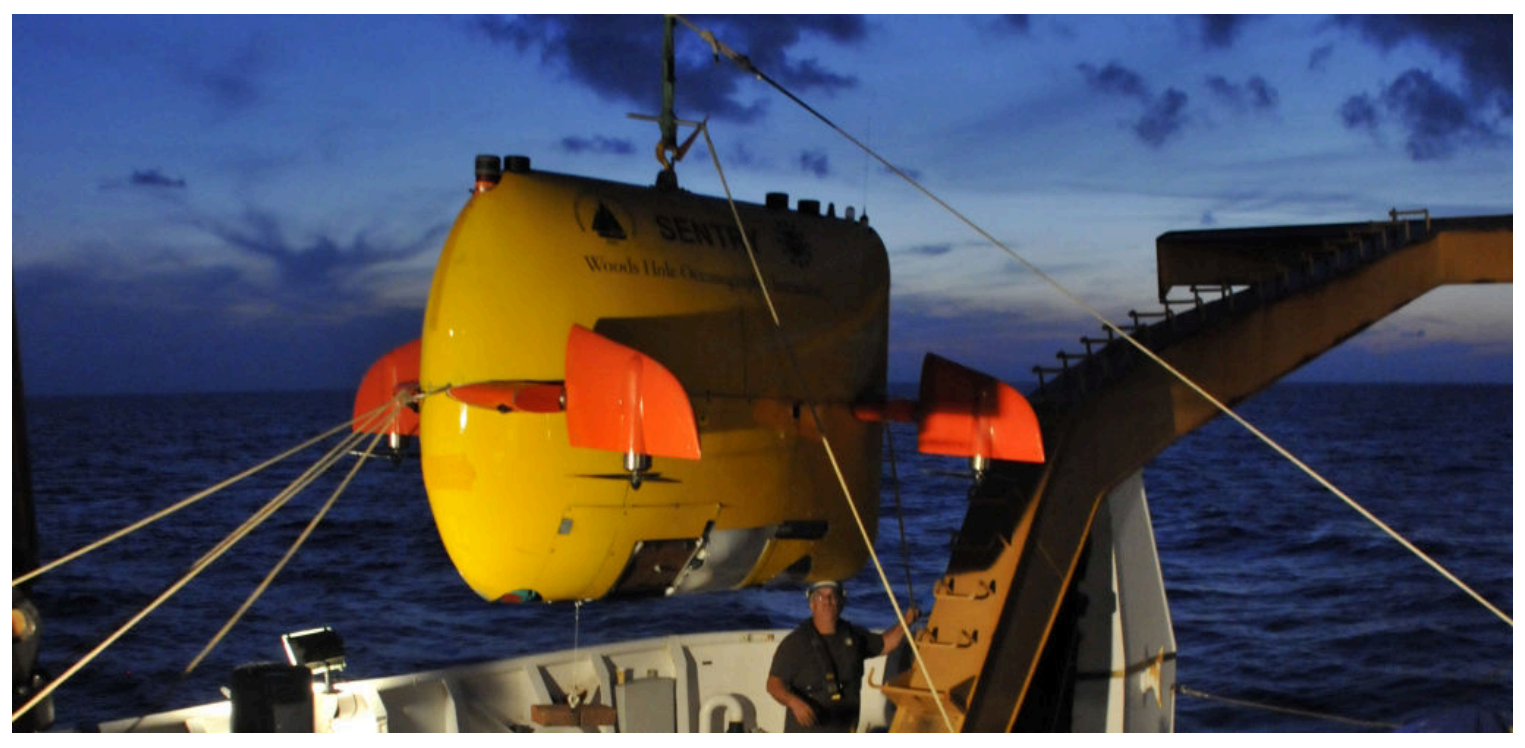

Figure 0-2: NDSF AUV Sentry being raised for launch. Two pairs of control fins with thrusters enable close bottom-following. The large separation between the center of buoyancy and center of gravity makes Sentry very stable in pitch and roll-this is desirable for high resolution mapping with multibeam or camera.

photo credit: Dana Yoerger, WHOI ABE/Sentry group

aiding. Navigation data is postprocessed after a dive to fuse external position inputs before mapping timeseries data to geographic locations.

This high-quality navigation suite makes Sentry an ideal platform for evaluating alternate navigation methods and state estimators before implementing them on vehicles with lower quality sensors.

The standard science sensor suite includes: a conductivity/temperature probe, precision magnetometers, a $400 \mathrm{kHz}$ multibeam sonar, and a 12-bit color camera. Sentry serves the oceanographic community, with limited extra payload for systems provided by scientists on each cruise. The vehicle has also carried redox potential (eH) probes, dissolved oxygen sensors, an in-situ mass spectrometer, a sub-bottom profiler, a sidescan sonar, and a stereo camera system.

\subsection{Thesis Statement}

This research explores three related hypotheses with the goal of improving the realtime performance and increasing the level of automation in underwater navigation: 
1. Rotors in Geometric Algebra can be used to formulate an elegant, efficient, and stable realtime alignment estimator suitable for in-situ use on underwater vehicles.

2. Relative water current profile data collected by a vehicle-mounted acoustic Doppler current profiler can provide estimates of the global vehicle velocity and position during descent and ascent phases of a mission.

3. Mathematically rigorous treatment of measurement delays in multi-sensor fusion Kalman filters can be achieved through state augmentation and conditional time-varying models without altering the underlying framework.

\subsubsection{Objectives}

This research has several objectives related to each hypothesis above.

\section{Rotor identification}

- formulate an adaptive identifier using rotors in Geometric Algebra (GA) for sensor alignment

- prove asymptotic stability

- demonstrate rotation identification in simulation

- demonstrate DVL/FOG alignment identification

- using archival data from a laboratory ROV

- using data from an AUV deployed in the field

\section{Independent navigation during descent and ascent}

- formulate online bin average, least squares, and recursive least squares approaches

- collect data from AUV ascents 
- demonstrate dead reckoned ascent using data from an AUV deployed in the field

\section{Fusing delayed measurements}

- formalize an efficient model-driven approach that works within the Kalman filter (KF) framework

- characterize performance of delayed measurement fusion using a simulation of a canonical system

- demonstrate model-driven fusion of delayed acoustic positioning measurements using data from an AUV deployed in the field performing

- USBL-aided DR

- asynchronous LBL-aided DR

\subsection{Dissertation Structure}

This chapter has provided a high-level introduction to underwater navigation and an explicit statement of the thesis. The following chapters present each individual contribution in detail:

Chapter 1 Navigation sensor alignment

This is a basic problem in any multi-sensor system-any uncertainties here propogate through the navigation system to the final result. Here I propose a stable online method for in-situ alignment identification, and demonstrate it on data from underwater vehicles in the laboratory and in the field. (Some of this work is previously reported in [15].)

Chapter 2 Dead reckoning through the water column

The ability to link GPS at the surface to precision DR near the seafloor might enable new kinds of missions for AUVs. This chapter introduces a method to DR through the water column using water current profile data collected by 
an onboard sensor, and provides an autonomous navigation link between the surface and the seafloor without any dependence on a ship or external acoustic tracking beacons. (Some of this work is previously reported in [28, 29].)

Chapter 3 Model-driven delayed measurement fusion

In many state estimation applications, delayed measurements present an interesting challenge. Underwater navigation is a particularly compelling case because of the relatively long delays inherent in all available position measurements. Here I propose an entirely model-driven approach to delayed measurement fusion based on state augmentation with a mathematically rigorous treatment of the delay. (Some of this work is previously reported in [54].)

The last chapter of this dissertation briefly recapitulates the conclusions of each chapter, and highlights some promising areas for future work. 


\section{Chapter 1}

\section{Navigation Sensor Alignment Using Rotors}

\section{Hypothesis}

Rotors in Geometric Algebra can be used to formulate an elegant, efficient, and stable realtime alignment estimator suitable for in-situ use on underwater vehicles.

\subsection{Introduction}

Rotations are ubiquitous in robotics and engineering. Two examples are: industrial robot arms move by rotating many different single degree-of-freedom joints, and the misalignment between different sensors is the rotation between their internal reference frames. We use Geometric Algebra (GA) to formulate a novel adaptive identifier for unknown rotations, and apply it to an important problem in underwater navigation.

We develop this identifier using rotors, which provide an elegant and efficient treatment of rotations (in multiple dimensions). More importantly, the GA formulation expose the geometric nature of the problem, and encourage an intuitive solution.

The remainder of this introduction motivates and explicitly defines the general problem of identifying an unknown three-dimensional rotation, then reviews existing approaches to rotation identification and other related work. To keep this paper 
self-contained, section 1.2 provides a brief primer on the basics of GA, which may be unfamiliar to many readers. This can be considered a section on the mathematical preliminaries for our approach-we only use GA basics in the formulation and stability analysis of the identifier. Section 1.3 details the rotor identifier, including the formulation, geometric intuition, a proof of asymptotic stability, and simulation results. In section 1.4, we apply the new identifier to estimate the fine misalignment between two important sensors in underwater navigation. We translate in-situ measurement data into a form usable by the identifier using integration by parts. We then demonstrate and characterize the performance of the identifier using data from a remotely operated vehicle (ROV) in controlled laboratory conditions (Section 1.5), and using field data from an autonomous underwater vehicle (AUV) operating in the deep ocean (Section 1.6).

\subsubsection{Motivation}

This study is motivated by a common practical problem-identifying the unknown alignment of two sensors using only the measurements from those sensors. This problem is of particular importance in robotic vehicle navigation, where data from many separate sensors must be fused in a common frame of reference. In dead reckoning, for example, a small alignment error leads to a large systematic position error in time. Accurate identification of sensor alignment is therefore of utmost importance, especially in applications like underwater navigation, where position updates may be severely limited.

\subsubsection{Problem: to identify a rotation from a pair of vectors}

Consider two misaligned sensors that provide vector measurements. If the measurement from the first sensor is $u$ and the measurement of the second sensor is $y$, then the relationship between the measurements is written:

$$
y=\underline{\mathrm{R}}(u),
$$




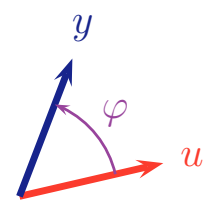

(a)

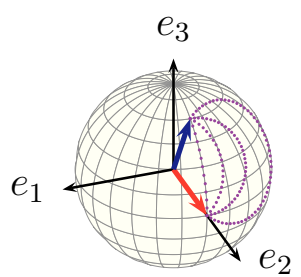

(b)

Figure 1-1: The rotation between two vectors in two dimensions (a) is described by a single parameter, the angle $\varphi$. The rotation is inherently constrained to the two-dimenstional plane. Identifying this rotation becomes more difficult in three dimensions-the plane of rotation is no longer constrained by the space (b). The input vector (red) could rotate along an infinite number of paths (purple) to reach the output vector (blue).

where $\underline{\mathrm{R}}(\cdot)$ is a linear operator that describes the unknown rotation between the coordinate frames of the sensors. The goal in aligning the measurements is to identify the unknown $\underline{\mathrm{R}}(\cdot)$. This problem is simple in two dimensions-the rotation is fully described by a single parameter (Figure 1-1a). But it is important to note the problem is underconstrained in higher dimensions-two vectors in 3-space do not uniquely identify a rotation (Figure 1-1b).

Given enough measurements, this problem can be solved in Linear Algebra (LA) using a conventional ordinary least squares (LS) approach to estimate the matrix that best transforms the inputs $u$ into the outputs $y$ :

$$
y=\mathbf{M} u
$$

but the result may not technically describe a rotation. Ordinary LS makes no guarantee that $\mathbf{M}$ will be a rotation matrix.

If the input/output relationship is restricted to rigid body rotations, the solution must be part of the special orthogonal group.

$$
y=\mathbf{R} u: \quad \mathbf{R} \in S O(3) .
$$

In three dimensions, this is a subgroup of $3 \times 3$ matrices subject to two constraints:

orthogonality columns of the matrix are orthogonal (i.e., independent) 
normality scale and chirality ${ }^{1}$ are preserved

These constraints define the group of rotation matrices:

$$
S O(3) \equiv\left\{\mathbf{R}: \mathbf{R} \in \mathbb{R}^{3 \times 3}, \mathbf{R}^{T} \mathbf{R}=\mathbf{I}_{3 \times 3}, \operatorname{det}(\mathbf{R})=1\right\}
$$

The goal of the alignment problem in LA is to identify the matrix that best transforms $u$ into $y$, subject to the constraints of the special orthogonal group.

We propose a novel alternative approach using GA and encoding the rigid body transformation in a rotor. The rotor acts on any element of GA by a double-sided application of the geometric product:

$$
y=R u \tilde{R}, \quad u=\tilde{R} y R, \quad R \in \operatorname{Spin}(3) .
$$

The algebraic structure of rotors inherently constrains all estimates to the group of rigid-body rotations. The approach presented here also provides a clear interpretation of the identifier using the first-order kinematics of the rotor estimate.

\subsubsection{Related Work}

This work is motivated by the practical problem of in-situ estimation of navigation sensor alignment for dead reckoning (DR) for underwater vehicles. The problem of Doppler velocity log (DVL)/fiber-optic gyroscope (FOG) alignment was first addressed by Kinsey \& Whitcomb in $[13,55]$. They use measurements from an external positioning system to observe the output of the DR plant. Using integration by parts, they are able to write the plant in the identifier form (1.3), and then apply standard and novel techniques to estimate the alignment.

This problem is a specific example of the more general problem of estimating rigid body rotations from a collection of uncertain data. Approaches to this problem can be loosely divided into batch methods and online methods. Much of the previous

\footnotetext{
${ }^{1}$ i.e., right-handed or left-handed. This mathematical property is also sometimes called orientation, but we will reserve that term to describe rotational position since that is more in line with its common use in geometry and navigation.
} 
literature has studied the problem using LA, but there are also some examples of batch methods in GA. This section summarizes the related work in the literature.

\section{Linear algebra, batch methods}

Standard approaches to identify the matrix transforming input data to output data include ordinary least squares and recursive least squares methods [56]. These methods are well-studied and their behavior is well characterized, but the solution is not guaranteed to describe a rigid body rotation. That is, these methods may not actually give a rotation matrix.

To enforce the orthogonality constraint on the LS solution, Arun et. al. introduce an application of singular value decomposition (SVD) in [57].

Umeyama adapted the LS-SVD approach in [58] to also enforce the normality constraint. The LS solution is now fully constrained to $S O(3)$. This constrained least squares (CLS) method is the present standard batch method for rotation identification.

In $[13,55]$, Kinsey \& Whitcomb apply the LS-SO(3) batch method to estimate the alignment between DVL and FOG in underwater navigation. The DR trajectories calculated using the estimated alignment agreed with long baseline (LBL) observations much better than those calculated using a rough visual alignment.

\section{Linear algebra, online methods}

The rotation identification problem is closely related to the attitude control problem. Bullo \& Murray study control on $S O(3)$ and $S E(3)$ using Lie groups ${ }^{2}$ in [60]. They recognize the geodesic length as a natural error metric on $S O(3)$. Assuming full state feedback, they use Lyapunov theory to prove the stability of a family of proportional and proportional-derivative control laws.

In $[14,61]$ Kinsey \& Whitcomb report an adaptive identifier on $S O(3)$, and prove its asymptotic stability using Lyapunov theory. They compare its performance to the

\footnotetext{
${ }^{2}$ Lie groups are actually closely related to rotors, and any Lie algebra can be expressed as a bivector algebra [59].
} 
LS methods in $[13,55]$ with favorable results.

More recently, Troni \& Whitcomb have applied the LS methods and adaptive identifier to identify the alignment between DVL and inertial navigation system (INS) [62,63]. This method no longer requires position measurements, so it has no dependencies external to the vehicle, making it a strapdown solution. Instead, it makes the mild assumption that the accelerometers and gyrocompass in the INS are internally aligned and calibrated. This extra assumption is almost trivial since it is done in manufacturing and does not need to be done in situ.

\section{Geometric algebra, batch methods}

Batch methods for rotor identification in GA have also been developed recently. Doran studied the estimation of an unknown rotor from noisy data in the context of camera localization in [64]. Buchholz and Sommer investigate the related problem of averaging in Clifford groups [65].

\subsubsection{Contributions}

The online GA method detailed in this chapter is an asymptotically stable adaptive identifier and was originally proposed in [15]. This method works recursively on input/output vector pairs and it uses a more compact and efficient encoding of rotations than the LA methods. Furthermore, since rotors are inherently constrained to represent rigid-body motions, no extra steps are required to constrain the solution. The rotor identifier is similar to the $S O(3)$ adaptive identifer developed in [14], but the GA formulation provides more meaningful and intuitive error measures (Section 1.3.2) than LA approaches. This encourages a geometric understanding of the problem and leads to a straightforward kinematic identification algorithm.

Table 2.1 compares this research to the related literature. The main contribution of this work is the formulation of an online adaptive identifier using rotors. One of the main advantages of using GA is the clarity it brings to the mathematical formulation of the identifier and the proof of asymptotic stability ${ }^{3}$. The GA formulation

\footnotetext{
${ }^{3} \mathrm{~A}$ proof of exponential stability would require a complete observation of the rotation for each
} 


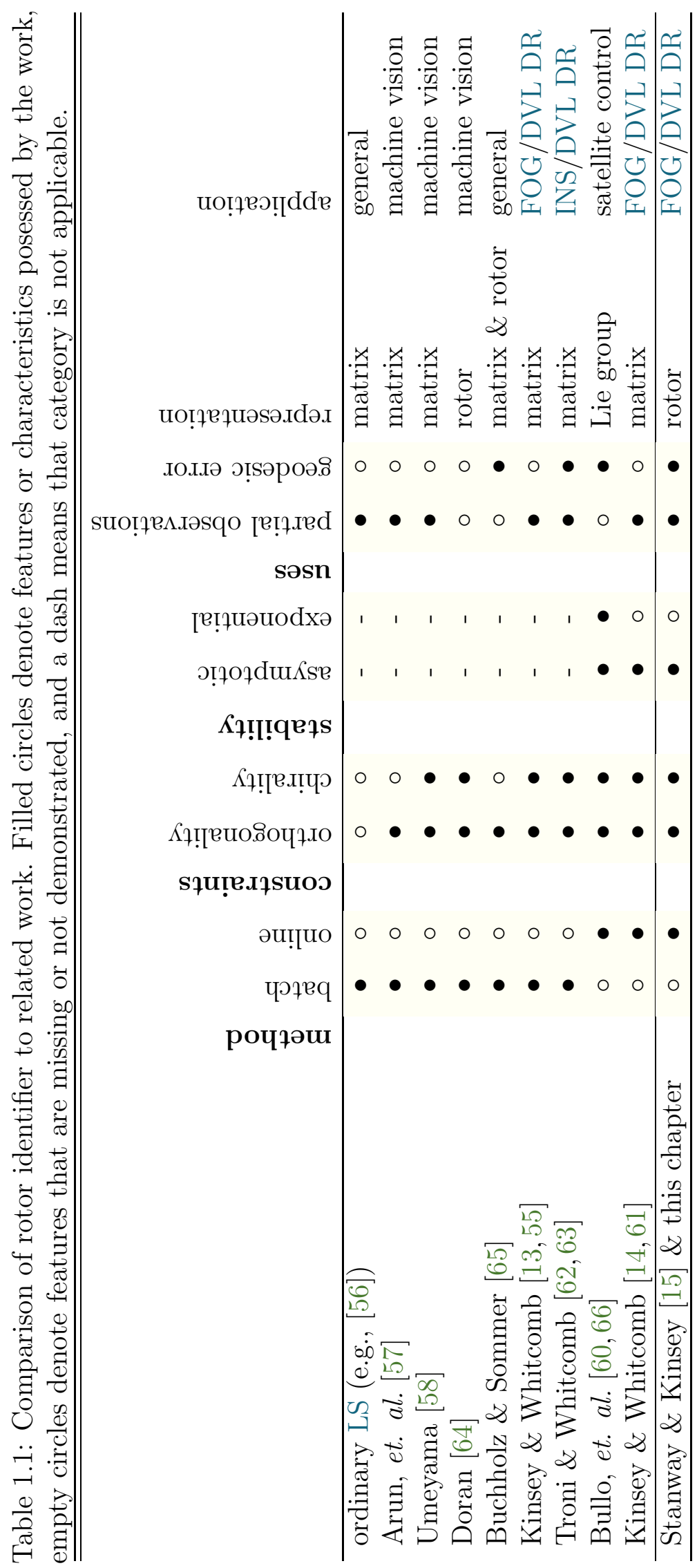


makes it easier to see how a simple proportional feedback regulator on the observable output error can provide asymptotically stable identification. This clarity encourages a more geometric understanding of the problem, and may simplify enhancements and extensions of the identifier in the future.

\subsection{Geometric Algebra Primer}

This section provides an introduction to the GA tools used in this dissertation. It is included because many readers may not be familiar with GA. It can be viewed as a section on the mathematical preliminaries necessary to formulate the rotation identifier and prove its stability. We only need the very basics of GA to do this.

For a more complete introduction to GA, the reader should refer to the introductory texts by Hestenes [67], Doran \& Lasenby [68], or Dorst, Fontijne, \& Mann [69]. Gull, et al., also provide an excellent short introduction in [70], and Bayro-Corrochano discusses several engineering applications in [71-74]. Here, we will only cover the very basics necessary for multiplying vectors and for representing rotations.

\subsubsection{Multiplying Vectors}

Every vector has two intrinsic properties: magnitude and direction. The products defined in GA describe relationships between these properties. We will define the inner product, the outer product, and the geometric product. These products then expand the language of GA to include elements of lower and higher grade (i.e., dimension), and even elements of mixed grade.

update of the estimate. Bullo assumes this in [60], but we cannot assume this in the FOG/DVL alignment problem that we apply the rotor identifier to in this research, because the observation of the rotation comes from pairs of input/output vectors (see section 1.1.2). 


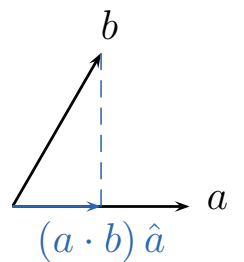

(a)

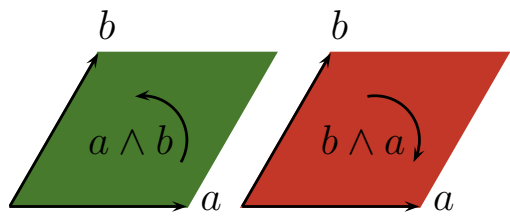

(b)

Figure 1-2: The geometric product is the sum of symmetric and antisymmetric parts: the inner product of two vectors (a) is symmetric and produces a scalar, the outer product of two vectors (b) is antisymmetric and produces a bivector.

\section{Inner Product}

We will start with a familiar concept-the inner product of two vectors is commutative, and defines a scalar $\alpha$ (grade 0$)$ :

$$
a \cdot b=b \cdot a=\alpha=|a||b| \cos \varphi
$$

where $\varphi$ is the angle between $a$ and $b$. The inner product vanishes if the vectors are perpendicular, but if they are parallel, it becomes the product of the magnitudes. This provides one way to define the magnitude of a vector:

$$
|a|=\sqrt{a \cdot a}
$$

This inner product should be familiar to the reader since it is essentially the same as in Linear Algebra.

\section{Outer Product}

The outer product of two vectors is anticommutative and defines a new entity called a bivector ${ }^{4} B$ (grade 2$)$ :

$$
a \wedge b=-b \wedge a=B=|a||b| \sin \varphi \hat{B}
$$

\footnotetext{
${ }^{4}$ Bivectors in GA are analogous in many ways to skew-symmetric matrices in LA, and both are related to Lie algebras.
} 
This bivector can be thought of as a directed plane segment in much the same way as a vector is a directed line segment. Unit bivectors, like $\hat{B}$, define a direction and have a magnitude of one. New bivectors can be generated by addition or subtraction.

The outer product is associative and can be used to build up even higher-grade elements, like trivectors $T$ (grade 3 ):

$$
(a \wedge b) \wedge c=a \wedge(b \wedge c)=T=\beta I .
$$

Trivectors are the highest grade element in $\mathbb{G}_{3}$, the three-dimensional Euclidean model of GA. Every trivector can be written as a scalar (e.g. $\beta$ ) times the unit trivector, so the unit trivector is called the pseudoscalar and denoted by $I$. Since no set of four or more vectors in $\mathbb{G}_{3}$ is independent ${ }^{5}$, the outer product of four or more vectors is always zero.

\section{Geometric Product}

The inner and outer products complement each other: one lowers the grade while the other raises it, one is commutative while the other is anticommutative. They are combined in the geometric product:

$$
a b=a \cdot b+a \wedge b=b \cdot a-b \wedge a=\alpha+B=M,
$$

which produces a multivector $M$ that contains both scalar and bivector parts.

The geometric product is actually the most basic product in GA. The inner and outer products can be derived axiomatically as the symmetric and antisymmetric components of the geometric product $[67,68]$.

$$
\begin{aligned}
a \cdot b & =\frac{1}{2}(a b+b a) \\
a \wedge b & =\frac{1}{2}(a b-b a)
\end{aligned}
$$

\footnotetext{
${ }^{5}$ This is true for any three-dimensional linear space.
} 
The geometric product itself is generally neither commutative nor anticommutative. But reversing the order of factors is still an important operation, known as reversion:

$$
\operatorname{reverse}(a b)=b a=(a b)^{\sim}=\tilde{M} \text {. }
$$

Sometimes it is useful to separate a multivector into parts of just one grade (i.e., select only the grade 2 part, the bivector). This operation is called grade selection, and denoted by angle brackets with the grade as a subscript:

$$
\langle M\rangle_{0}=\alpha, \quad\langle M\rangle_{2}=B
$$

In general, a multivector can be a sum of elements of all grades: $A=\alpha+a+B+\beta I$. It may seem odd at first to add together elements of different grades, but it is really no different than adding together the real and imaginary parts of a complex number [70]. In fact, the constructs of GA are closely related to complex numbers and quaternions, which brings us to the topic of rotations.

\subsubsection{Rotating vectors}

Rotations in GA are encoded by special elements called rotors. These describe a rotation in a plane, rather than around an axis-this extends more readily to spaces that are not 3D Euclidean (i.e., 2D plane, 4D projective, or 5D conformal). Any multivector can be transformed using rotors: simply left-multiply by the rotor, and right-multiply by its reverse:

$$
M^{\prime}=R M \tilde{R}
$$

This double-sided application of the geometric product is sometimes called the versor or sandwich product. This transformation works equally well in two dimensions, three dimensions, or even more ${ }^{6}$.

\footnotetext{
${ }^{6}$ In fact, rotors in Conformal Geometric Algebra describe general rigid body motions, encoding both rotation and translation in a single entity $[68,75]$.
} 


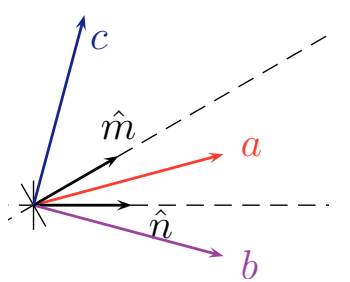

(a)

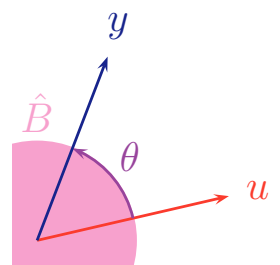

(b)

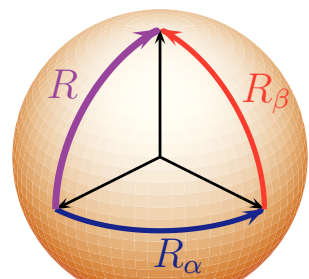

(c)

Figure 1-3: Three main methods of composing rotors: (a) from two vectors using the concept of repeated reflections, (b) from a bivector using the exponential map, or (c) from two or more other rotors as a chain.

\section{Definition}

The set of rotors, or proper unitary spinors, is given by the normalized elements of the even subalgebra $\mathbb{G}^{+}$. In a three-dimensional Euclidean space, they form the group:

$$
\operatorname{Spin}(3) \equiv\left\{R: R \in \mathbb{G}_{3}^{+}, R \tilde{R}=\tilde{R} R=1\right\}
$$

which is isomorphic to the quaternions $\mathbb{H}$, but with emphasis on geometric interpretation and easily extended to higher dimensional spaces. Spin(3) provides a double-cover over $S O(3)$.

Three-dimensional rotors have four coefficients: one scalar and three bivectors. The orthogonality constraint is implicit in the structure. The unit norm constraint is simple to enforce, but often unneccesary because of good numerical stability properties [76-78]. In contrast, rotation matrices have nine coefficients, and orthonormality is more difficult to ensure.

\section{Composition}

There are three main methods to compose a rotor (Figure 1-3): (i) using the concept of repeated reflections, (ii) as the geometric exponential of a bivector, or (iii) chaining together a sequence of multiple rotors. We use all of them in the formulation and stability proof of the identifier. 


\section{Repeated reflections}

The Cartan-Dieudonné theorem states that any Euclidean transformation can be expressed by a combination of reflections [79]. This suggests the first method of rotor composition: repeated reflections.

The sandwich product using a unit vector on each side:

$$
z=\hat{n} u \hat{n} .
$$

produces a reflection. Any combination with an even number of reflections will produce a proper ${ }^{7}$ rotation. So rotors are the geometric product of an even number of unit vectors:

$$
y=R u \tilde{R}=\hat{m} \hat{n} u \hat{n} \hat{m} .
$$

An even number of repeated reflections (as opposed to a single reflection) are required to ensure right-handed coordinates remain right-handed.

This method of composing a rotor is particularly useful when you need to calculate the 'shortest' rotor between two vectors. Choose the first unit vector in the direction of the first vector. Then add the two vectors and normalize the sum to get the second unit vector. This one points in the direction that bisects the angle between the original vectors, as shown in Figure 1-3a.

\section{Bivector exponential}

In the second rotor composition method, a bivector encodes the plane and angle (i.e., the direction and magnitude) of the rotation. The rotor that generates this rotation is the exponential ${ }^{8}$ of the bivector:

$$
R=\exp \left(-\frac{1}{2} \theta \hat{B}\right)=\mathrm{e}^{-\frac{1}{2} B}
$$

\footnotetext{
${ }^{7}$ Conversely, an odd number of reflections produces an improper rotation, or rotoreflection.

${ }^{8}$ The concept of an exponential function is easily generalized to operate on multivectors, see [67] or $[68]$.
} 
where the factor of $1 / 2$ is because we multiply the rotor twice (once on each side) and the negative sign is a convention so that positive angles produce a counterclockwise (i.e., right-handed) rotation.

It is also useful to define a multivector logarithm so that the bivector $B$ can be determined from the rotor $R$. This actually has some subtle points, but is well-defined for rotors. We adopt the definition given in [67] and denote the rotor logarithm:

$$
B=-2 \ln R
$$

\section{Rotor chains}

The rotor group is closed under multiplication with the geometric product. This leads to a third way to compose rotors-as a chain of two or more other rotors:

$$
R_{a}\left(R_{b} u \tilde{R}_{b}\right) \tilde{R}_{a}=\left(R_{a} R_{b}\right) u\left(\tilde{R}_{b} \tilde{R}_{a}\right)=R_{a b} u \tilde{R}_{a b}
$$

The geometric product of two rotors will always produce another rotor. This property is very useful for discrete-time kinematics.

GA is a much richer topic-the interested reader should refer to texts referenced at the beginning of this section. Having covered the very basics, we now have all the tools we need to build our identifier and study its properties.

\subsection{Asymptotically Stable Rotor Identifier}

Here we propose an adaptive identifier based on first-order rotor kinematics and a geometric interpretation of identification error. We will begin by defining the rotation plant in terms of input and output vectors. Then we discuss several possible error metrics, with emphasis on the geometric meaning of each of them. We use firstorder rotor kinematics and a proportional gain error-state feedback regulator (ESFR) to formulate a rotor identifier, then prove its asymptotic stability using Lyapunov theory. This section concludes with a brief discussion of gain selection. 


\subsubsection{Plant Definition}

Given known input $u$ and output $y$ signals of the same grade ${ }^{9}$, the plant:

$$
y=R u \tilde{R}
$$

describes the rotation from input to output. The unknown rotor $R \in \operatorname{Spin}(3)$ is a stationary element of the even subalgebra, and encodes a rigid body rotation. The goal of the method proposed here is to identify this unknown rotor, $R$.

We will define the time-varying estimate of the rotor as $S(t) \in \operatorname{Spin}(3)$. The expected plant output is then:

$$
v=S u \tilde{S}
$$

We will call (1.22) the identification plant.

The goal of the identifier is to drive the estimated rotor $S$ toward the actual rotor $R$. Since each timestep provides an incomplete observation of the actual rotor, we achieve this using feedback on the output error. The action of the identifier drives the expected output $v$ toward the observed output $y$.

\subsubsection{Error Metrics}

This problem has two types of error: the parameter error describes the difference between the estimated and actual values; the output error describes the difference between the estimated and observed outputs. These errors can be parameterized in several ways-GA encourages a geometric interpretation of each parameterization and of the relationships between them. Defining an error rotor is natural because the goal is to identify a rotor in the first place. A bivector error can be directly applied to drive the first order kinematics as a rotational velocity. Finally, a scalar error is most convenient for summarizing performance and in Lyapunov analysis. Here we

\footnotetext{
${ }^{9}$ We focus on vector signals $\left(u, y \in\left\langle\mathbb{G}_{3}\right\rangle_{1}\right)$ for the remainder of this paper. However, the methods presented here should apply equally well to bivectors or to higher-grade multivectors, so long as the grade of the output is the same as the grade of the input-otherwise (1.21) and (1.22) would not describe rotations.
} 
define these three error metrics for both types of error. We discuss their geometric interpretation and the relationships between them.

\section{Parameter Error}

Define the parameter error rotor:

$$
Q=R \tilde{S}: Q \in \operatorname{Spin}(3)
$$

This definition gives the 'difference' between the estimated rotor and the actual rotor. Applying the parameter error rotor directly to the estimated output will rotate it into the actual output:

$$
\begin{aligned}
Q v \tilde{Q} & =(R \tilde{S})(S u \tilde{S})(S \tilde{R}) \\
& =R u \tilde{R}=y
\end{aligned}
$$

The error rotor is the geometric operator that resolves the difference between the identification plant and the actual plant.

The parameter error bivector is derived from the natural logarithm of the parameter error rotor:

$$
X=-2 \ln (Q): Q=\mathrm{e}^{-\frac{1}{2} X}
$$

This metric encodes both the magnitude and direction of the error, which can be interpreted as the angle and the rotation plane.

The magnitude of the parameter error is a scalar:

$$
\chi=\|X\|^{1 / 2}=\sqrt{X \tilde{X}}
$$

This is the natural distance measure on $\mathbb{S}^{3}$, and concisely summarizes the error state. It is distilled down to the angle between the estimate and the truth, and is analogous to the distance measure defined in [60]. As a scalar, $\chi$ is convenient for visualization and for stability analysis, but the lack of directional information makes it less useful 
for feedback.

\section{Output Error}

Since a pair of input/output vectors does not uniquely define a rotation, the parameter error is only partially observed at each timestep. We define output errors to characterize the observable part of the misalignment.

The output error rotor is defined:

$$
P=\hat{m} \hat{n}=\frac{y+v}{|y+v|} \frac{v}{|v|}: P \in \operatorname{Spin}(3)
$$

so that it rotates the estimated output $v$ directly into the measured output $y$ along the shortest path on the sphere ${ }^{10}$. Note that (1.26) uses the concept of repeated reflections (1.17) to compose $P$.

The output error bivector is defined analogously to its parameter error counter$\operatorname{part}^{11}$ :

$$
Y=-2 \ln (P)
$$

This bivector form of the output error encodes information on both magnitude and direction-we will show in section 1.3.3 how it can be used as a rotational velocity to drive the identification plant kinematics.

Similar to (1.25), the output error angle is:

$$
\psi=\|Y\|^{1 / 2}=\sqrt{Y \tilde{Y}}
$$

Again, this scalar provides a useful summary of the parameter error, but the lack of directional information makes it unsuitable for feedback.

Each of these error measures is valid, and each has a different use and interpre-

\footnotetext{
${ }^{10}$ In [69], Dorst gives an alternate form for the 'shortest' rotor between two vectors: $R=(1+b a) / \sqrt{2(1+a \cdot b)}$ This is equivalent to (1.26) for $a=v$ and $b=y$.

${ }^{11}$ There are, of course, alternate ways to define the output error bivector. We have chosen to define $Y$ this way for consistency with $X$ in (1.24) and so that its magnitude leads directly to the arc length of the error. Moreover, (1.26) normalizes the vectors $y+v$ and $v$ to emphasize the difference in direction and not magnitude. This is one difference from the approach in [14].
} 
tation. Note that the error rotors tend toward unity as $S \rightarrow R$, while the bivectors and angles tend toward zero.

\section{Partial observation and error bivectors}

The output error bivector is only part of the parameter error bivector-there may also be an unobserved component, $Z$, orthogonal to the observed output error. This is because a rotation defined only by two vectors is not unique, as noted earlier in section 1.1.2. The parameter error bivector is the sum of these two parts:

$$
X=Y+Z, \quad \text { where: } \quad Z \perp Y .
$$

Note that the orthogonality condition is a weak constraint on the unobserved component$Z$ can have any magnitude ${ }^{12}$ or direction as long as it is not parallel to $Y$.

Despite this limitation, a straighforward proportional gain feedback regulator on the observed output error is an asymptotically stable adaptive identifier.

\subsubsection{Proportional Gain Identifier}

Now that we have a clear understanding of the underlying geometry of this problem, we can formulate a simple identifier based on proportional feedback into the first order kinematics of the system.

\section{First-Order Kinematics}

We are interested in the time derivative of the rotor estimate $S(t) \in \operatorname{Spin}(3)$. Recall the exponential parameterization of the rotor:

$$
S(t)=\mathrm{e}^{-\frac{1}{2} B(t)}
$$

\footnotetext{
${ }^{12}$ Strictly speaking, the bivector errors may have any magnitude. In practice, this is limited to the interval $[0,2 \pi)$.
} 
Since $B$ describes the rotation angle, $d B / d t$ describes the rotational velocity. We will adopt the notation:

$$
\dot{B}=\Omega=I \omega
$$

where $\Omega$ is the natural bivector form of the rotational velocity. The dual form, with rotational velocity encoded by a vector $\omega$ multiplied by the pseudoscalar $I$, may be more familiar to some readers.

Direct application of the chain rule gives the first order rotor differential equa$\operatorname{tion}^{13}$ :

$$
\begin{aligned}
\frac{d}{d t}(S(t)) & =\frac{d B}{d t} \frac{d S}{d B} \\
\dot{S} & =-\frac{1}{2} \Omega S .
\end{aligned}
$$

We can now use the output error bivector $Y$ defined in (1.27) as a rotational velocitiy to drive the kinematics of the identification plant (1.22).

\section{Identifier update law}

We formulate the rotor identifier as an output error feedback regulator with proportional gain:

$$
\Omega(t)=\kappa_{p}(t) Y(t): \kappa_{p}(t)>0 \forall t
$$

The continuous-time update law becomes:

$$
\dot{S}=-\frac{\kappa_{p}}{2} Y S, \text { and } \dot{\tilde{S}}=\frac{\kappa_{p}}{2} \tilde{S} Y
$$

Error due to finite precision numerical integration is of little concern-the algebraic structure of the rotor ensures that it remains orthogonal, and the unit norm constraint can be enforced by periodic renormalization if necessary. This chapter also provides an exact discrete-time update in a later section.

\footnotetext{
${ }^{13}$ Hestenes gives an indirect calculation in [67], initially solving for the rotational velocity expressed in a different reference frame.
} 


\section{Parameter Error Evolution}

The evolution of the estimate is characterized by the time derivative of the parameter error rotor:

$$
\dot{Q}=\dot{R} \tilde{S}+R \dot{\tilde{S}}=R \dot{\tilde{S}}
$$

Substituting the update law (1.35) gives the continuous-time system:

$$
\dot{Q}=R\left(\frac{\kappa_{p}}{2} \tilde{S} Y\right)=\frac{\kappa_{p}}{2} Q Y
$$

This looks very similar to the first-order rotor differential equation (1.33). In fact, (1.37) simply states that the rotational velocity of the parameter error is proportional and opposite to the rotational velocity of the estimate.

$$
\begin{aligned}
\dot{Q} & =-\frac{1}{2} \dot{X} Q \\
-\dot{X} & =2 \dot{Q} \tilde{Q}=\kappa_{p} Q Y \tilde{Q}=\Omega^{\prime},
\end{aligned}
$$

where $\Omega^{\prime}$ is the rotational velocity expressed in the reference frame of $R$.

\section{Discrete-time implementation}

Most applications will provide measurements of the input $u$ and the output $y$ in discrete time, so it makes sense to develop a discrete-time version of the proportional update law (1.35).

Define the discrete update of the rotor estimate:

$$
\delta S=S_{k} \tilde{S}_{k-1}: S_{k}=(\delta S)\left(S_{k-1}\right)
$$

Assuming the gain and rotational velocity of the rotor estimate are constant over the timestep, we use Euler's method to integrate (1.35) directly:

$$
\delta S=\int_{0}^{\delta t}\left(-\frac{\kappa_{p}}{2} Y S\right) d \tau=\mathrm{e}^{-\frac{1}{2}\left(\kappa_{p} \delta t\right) Y}
$$




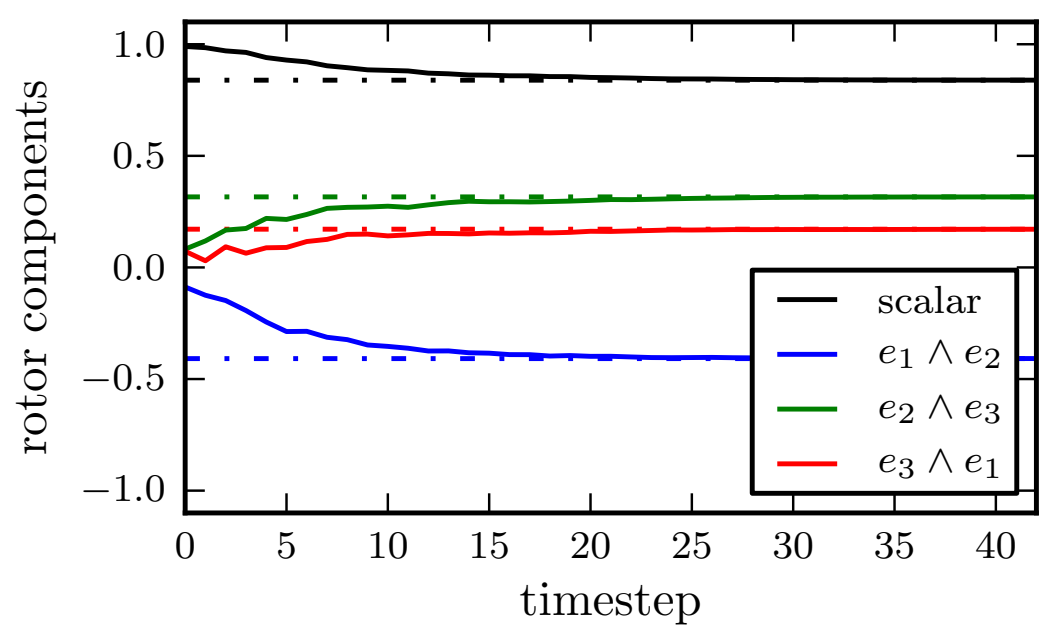

Figure 1-4: The components of the estimated rotor $S$ computed by the proportional gain rotor identifier converge to the components of the unknown rotor $R$ (marked by dash-dot lines).

This discrete-time form of the rotor identifier was implemented in $\mathrm{C}$ using a base library generated by Gaigen2.5 [80], the latest version of the GA implementation generator $[81,82]$.

Figure 1-4 shows the four components of the estimated rotor $S$ converging toward the true rotor $R$ in a simulation using the discrete-time rotor identifier. In other simulations, several levels of random Gaussian noise were added to the output $y$. Figure 1-5 shows how the scalar parameter error $\chi$ decreases in time until it reaches the level of the noise.

\subsubsection{Asymptotic Stability}

We employ Lyapunov theory $[83,84]$ to prove the stability of $(1.35)$. This involves identifying an energy-like scalar function of the error, then showing that the time derivative of this function does not increase. 


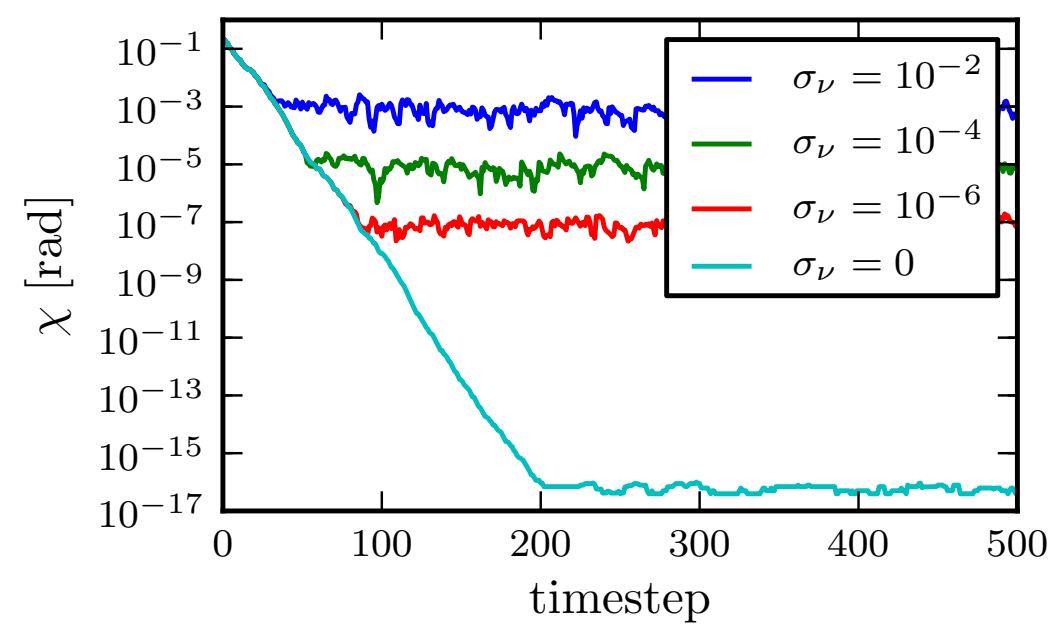

Figure 1-5: Evolution of the scalar parameter error when the input/output vector pairs are corrupted by additive white noise. The estimated rotor converges to the actual rotor until $\chi$ approaches the noise floor. The estimate then oscillates around the truth with magnitude controlled by the gain and the noise level.

\section{Lyapunov Candidate Function}

We choose a Lyapunov candidate using the natural distance measure on $\mathbb{S}^{3}$, which is the parameter error angle $\chi$ :

$$
V=\frac{1}{2} \chi^{2} .
$$

This function is analagous to the Lyapunov functions employed in [14,60]. By expanding the parameter error angle $\chi$ the Lyapunov candidate function can also be written directly in terms of the parameter error rotor:

$$
V=2 \ln Q \ln \tilde{Q},
$$

or the parameter error bivector:

$$
V=\frac{1}{2}\|X\|^{2}=\frac{1}{2} X \tilde{X} .
$$


This last bivector form will be most useful for studying the Lyapunov derivative, as detailed in the next section.

All forms of the Lyapunov candidate function (1.42) are equivalent and positive (semi-)definite ${ }^{14}$, and therefore satisfy the first requirement of a Lyapunov candidate. In this particular problem:

$$
\begin{aligned}
& V=0 \Longleftrightarrow Q=1, S=R, \text { and } \\
& V>0 \forall Q \neq 1, S \neq R .
\end{aligned}
$$

Note also that $\ln (\cdot)$ is defined over the entire sphere $\mathbb{S}^{3}$, even at $\pm n \pi$, so that this Lyapunov candidate is continuous and singularity-free.

\section{Lyapunov Derivative}

To prove asymptotic stability, we must show that the Lyapunov function decreases monotonically. We consider its time derivative:

$$
\begin{aligned}
\dot{V} & =\frac{d}{d t}\left(\frac{1}{2} X \tilde{X}\right) \\
& =\frac{1}{2}(\dot{X} \tilde{X}+X \dot{\tilde{X}})
\end{aligned}
$$

Recalling that the reverse of a bivector is its negative ${ }^{15}$, we obtain

$$
\dot{V}=-\frac{1}{2}(\dot{X} X+X \dot{X})
$$

This is the Lyapunov derivative.

$X$ and $\dot{X}$ do not commute in the geometric product, but since they are both bivectors (i.e., elements of the same grade), they can be decomposed into parallel and perpendicular parts:

$$
\dot{X}=\dot{X}_{\| X}+\dot{X}_{\perp X}
$$

\footnotetext{
14 Note that $\chi \geq 0$ already, so it is not strictly necessary to square it-we do so to encourage the intuition that this is like a potential energy.

${ }^{15}$ In $\mathbb{G}_{3}$, the three dimensional Euclidean Geometric Algebra. (This is not necessarily true in GAs with other signatures.)
} 
Since orthogonal (independent) bivectors anticommute:

$$
X \dot{X}_{\perp X}=-\dot{X}_{\perp X} X
$$

and parallel (dependent) bivectors commute:

$$
X \dot{X}_{\| X}=\dot{X}_{\| X} X
$$

the Lyapunov derivative can be rewritten:

$$
\begin{aligned}
\dot{V} & =-\frac{1}{2}\left(\left(\dot{X}_{\| X}+\dot{X}_{\perp X}\right) X+X\left(\dot{X}_{\| X}+\dot{X}_{\perp X}\right)\right) \\
& =-\dot{X}_{\| X} X=-\dot{X} \cdot X=-\langle\dot{X} X\rangle_{0},
\end{aligned}
$$

which reduces to a scalar, as expected. Substituting (1.39) gives the Lyapunov stability criterion:

$$
\dot{V}=\kappa_{p} Y_{\| X} X=\kappa_{p} Y \cdot X=\kappa_{p}\langle Y X\rangle_{0} \leq 0
$$

Note that the equality happens only when the output error bivector (and hence, the rotational velocity of the estimate) is orthogonal to the parameter error bivector.

This stability criterion is related to an extension of Gauss' Lemma given in [66]. It simply states that the component of rotational velocity which is not orthogonal to the error bivector must be parallel (and not antiparallel) to it. This condition is satisfied by the rotational velocity defined in (1.34) as a positive proportional gain applied to the output error bivector defined in (1.27). If the output error had been defined in the opposite sense, the stability criterion (1.52) would simply require that the proportional gain be negative instead of positive.

An alternate form of this stability criterion follows after recalling that the output error bivector is a partial observation of the parameter error bivector. Substitut- 
ing (1.29) into (1.46) gives:

$$
\dot{V}=-\frac{1}{2}(\dot{X}(Y+Z)+(Y+Z) \dot{X}) \text {. }
$$

Substituting the time derivative of the parameter error bivector (1.39) gives:

$$
\begin{aligned}
\dot{V} & =-\frac{1}{2} \kappa_{p}(Y(Y+Z)+(Y+Z) Y) \\
& =\frac{1}{2} \kappa_{p}(2 Y Y+Y Z+Z Y) .
\end{aligned}
$$

The last two terms cancel since the observed output error is orthogonal to the unobserved portion of the parameter error and orthogonal bivectors anticommute:

$$
\begin{aligned}
Z \perp Y & \Longrightarrow Z Y=-Y Z \\
& \Longrightarrow Z Y+Y Z=0
\end{aligned}
$$

So the Lyapunov derivative reduces to a function of the scalar output error:

$$
\begin{aligned}
\dot{V} & =\kappa_{p} Y Y=-\kappa_{p} Y \tilde{Y}=-\kappa_{p}\|Y\|^{2} \\
& =-\kappa_{p} \psi^{2} \leq 0 .
\end{aligned}
$$

This form of the stability criterion is equivalent to (1.52).

\subsection{Application to Dead Reckoning for Underwater Navigation}

The previous sections have motivated and developed an asymptotically stable rotor identifier with the intent of using it to estimate sensor alignment in-situ. This section identifies DR for underwater vehicle navigation as an example application, and details laboratory and field experiments to identify the rotation between reference frames of two critical sensors. We demonstrate improved navigation accuracy, characterized by 


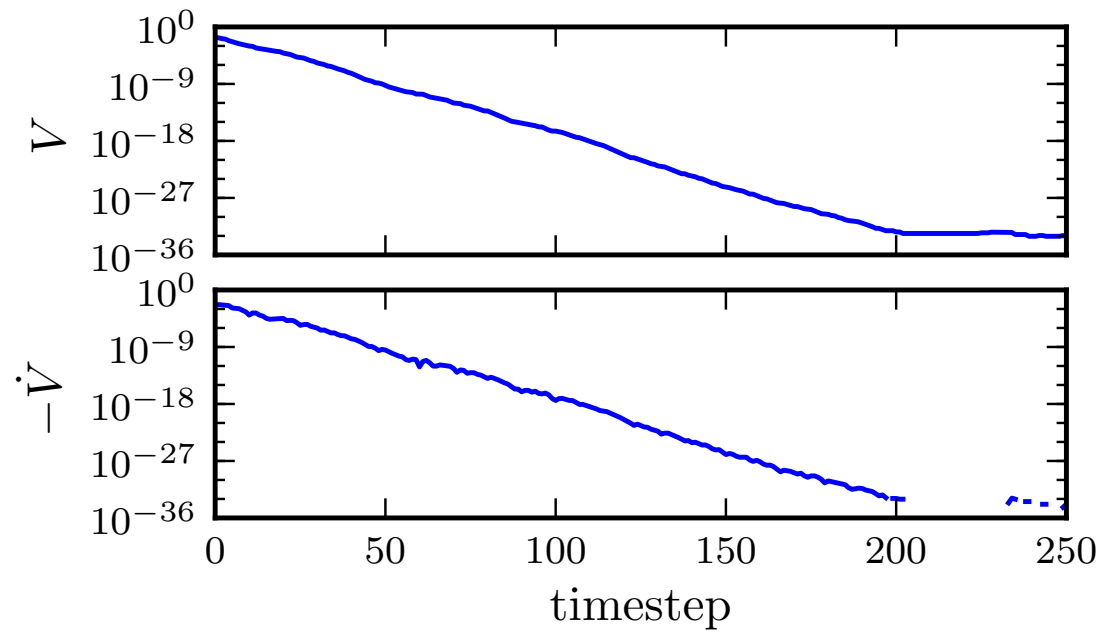

Figure 1-6: Time evolution of the Lyapunov function $V$ and its derivative $\dot{V}$. The Lyapunov function decreases monotonically as the rotor estimate $S$ approaches the actual rotor $R$, until the scalar error reaches the noise level of the measurements.

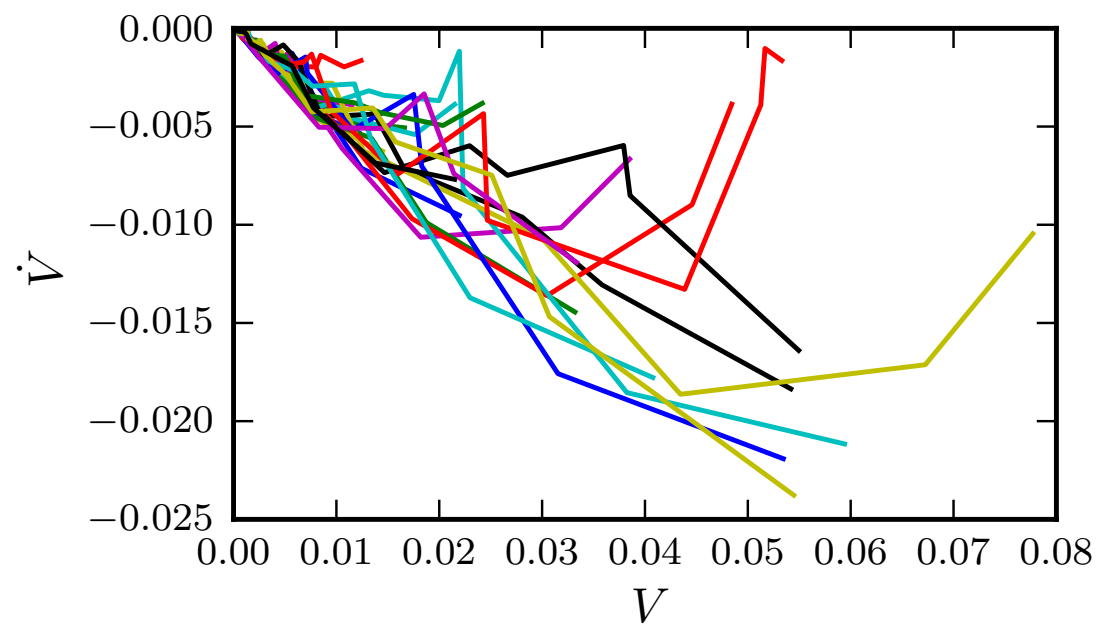

Figure 1-7: Phase-space trajectory of the Lyapunov function $V$ for twenty tests with random data. As the rotor estimate $S$ evolves in time, the system approaches equilibrium at the origin.

lower residuals in observations on the $\mathrm{DR}$ vehicle position. 


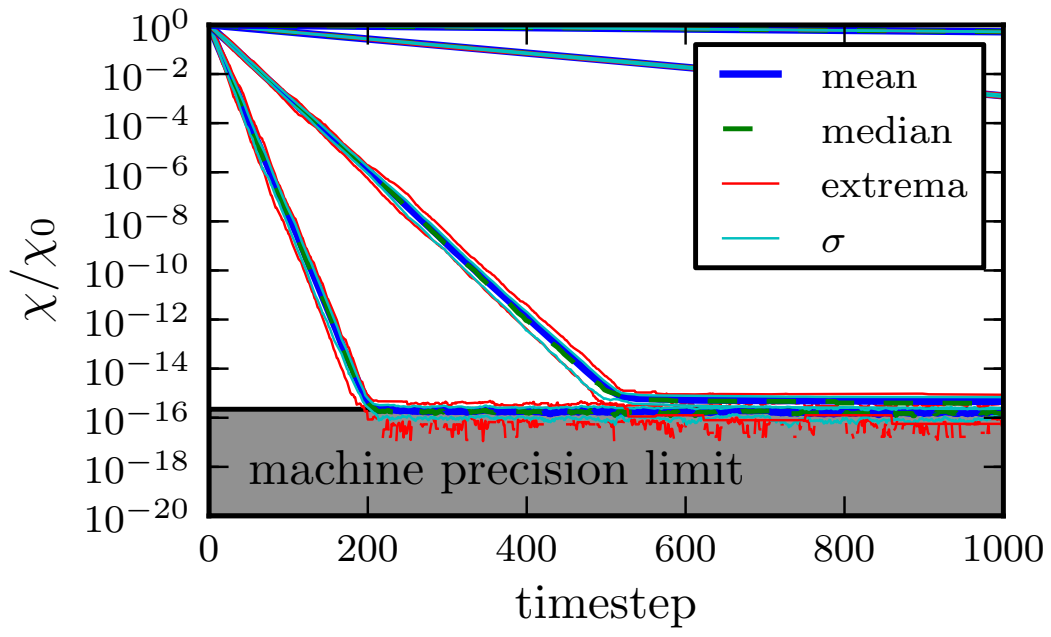

Figure 1-8: Evolution of the scalar parameter error, normalized by its initial value. Results are ensemble-averaged over twenty randomly generated datasets and compared for four proportional gains: $\kappa_{p} \in\{0.001,0.01,0.1,0.25\}$.

\subsubsection{Dead Reckoning and the Identification Plant}

\section{Position-velocity form using Linear Algebra}

The first-order kinematic equation for a vehicle moving in the navigation frame (denoted by a superscript ${ }^{n}$ ) is:

$$
\mathbf{p}^{\mathrm{n}}(t)=\mathbf{p}^{\mathrm{n}}(0)+\int_{0}^{t} \mathbf{u}^{\mathrm{n}}(\tau) d \tau
$$

where $\mathbf{p}$ is the position vector and $\mathbf{u}$ is the velocity vector.

Rotating the velocities into the vehicle frame (denoted by a superscript ${ }^{\mathrm{v}}{ }^{ }$) gives the first-order DR equation:

$$
\mathbf{p}^{\mathrm{n}}(t)=\mathbf{p}^{\mathrm{n}}(0)+\int_{0}^{t} \mathbf{R}_{\mathrm{n}}^{\mathrm{v}}(\tau) \mathbf{u}^{\mathrm{v}}(\tau) d \tau
$$


The DVL reports velocities in the instrument frame, so (1.61) is rewritten:

$$
\mathbf{p}^{\mathrm{n}}(t)=\mathbf{p}^{\mathrm{n}}(0)+\int_{0}^{t} \mathbf{R}_{\mathrm{n}}^{\mathrm{v}}(\tau) \mathbf{R}_{\mathrm{v}}^{\mathrm{i}} \mathbf{u}^{\mathrm{i}}(\tau) d \tau
$$

where $\mathbf{R}_{\mathrm{i}}^{\mathrm{v}} \in S O(3)$ is the matrix describing the time invariant rotation from instrument coordinates to vehicle coordinates. This is the rotation we are trying to identify.

The position of the vehicle in the navigation frame, $\mathbf{p}^{\mathbf{n}}$, is measured by LBL or ultra-short baseline (USBL). The rotation from the vehicle frame to the navigation frame is computed from the attitude measured by the North-seeking gyrocompass ${ }^{16}$, and can be expressed by the rotation matrix $\mathbf{R}_{\mathrm{n}}^{\mathrm{v}}(\tau)$. The DVL measures the vehicle velocity in the instrument frame, $\mathbf{u}^{\mathrm{i}}(\tau)$. We are left with the unknown sensor alignment, expressed by the rotation matrix $\mathbf{R}_{\mathrm{i}}^{\mathrm{v}}$.

The task now is to transform (1.62) with the available inputs into a form compatible with the identifier plant. Here we review the approach taken in $[13,14]^{17}$.

Differentiating (1.62) in time gives:

$$
\dot{\mathbf{p}}^{\mathrm{n}}(t)=\mathbf{R}_{\mathrm{n}}^{\mathrm{v}}(t) \mathbf{R}_{\mathrm{v}}^{\mathrm{i}} \mathbf{u}^{\mathrm{i}}(t)
$$

Rotating both sides of the equation into the vehicle frame:

$$
\mathbf{R}_{\mathrm{v}}^{\mathrm{n}}(t) \dot{\mathbf{p}}^{\mathrm{n}}(t)=\mathbf{R}_{\mathrm{v}}^{\mathrm{i}} \mathbf{u}^{\mathrm{i}}(t)
$$

brings us to the form of the identification plant.

However, the signal $\dot{\mathbf{p}}^{\mathrm{n}}(t)$ is not generally available in situ in the field. Numerical differentiation of LBL position measurements in the field is impractical due to delays and relatively high noise level. However, integrating the left hand side of (1.64) by

\footnotetext{
${ }^{16}$ This can be applied with any attitude/orientation reference (e.g. magnetic compass, attitude and heading reference system (AHRS), gyrocompass, FOG, ring-laser gyroscope (RLG), inertial measurement unit (IMU), or INS). The application in this research is to identify the misalignment between a FOG-based North-seeking gyrocompass and a DVL, so we will use the acronym FOG for brevity.

${ }^{17} \mathrm{~A}$ formulation of the DR or INS equations in GA may be an interesting topic for future research.
} 
parts gives:

$$
\int \mathbf{R}_{\mathrm{v}}^{\mathrm{n}}(\tau) \dot{\mathbf{p}}^{\mathrm{n}}(\tau) d \tau=\mathbf{R}_{\mathrm{v}}^{\mathrm{n}}(t) \mathbf{p}^{\mathrm{n}}(t)-\int \dot{\mathbf{R}}_{\mathrm{v}}^{\mathrm{n}}(\tau) \mathbf{p}^{\mathrm{n}}(\tau) d \tau+\text { const }
$$

This removes the unavailable signal $\dot{\mathbf{p}}^{\mathrm{n}}(\tau)$, but introduces the new parameter $\dot{\mathbf{R}}_{\mathrm{v}}^{\mathrm{n}}(\tau)$. The new signal may be measured directly by the FOG, or it may be numerically differentiated (since the update rate is higher, delay is negligible, and noise is lower for the FOG than with LBL).

Since $\mathbf{R}_{\mathrm{i}}^{\mathrm{v}}$ is stationary the integral of the right hand side of (1.64) is:

$$
\int \mathbf{R}_{\mathrm{v}}^{\mathrm{i}} \mathbf{u}^{\mathrm{i}}(\tau) d \tau=\mathbf{R}_{\mathrm{v}}^{\mathrm{i}} \int \mathbf{u}^{\mathrm{i}}(\tau) d \tau
$$

And now we have an equation in the form of the identifier plant using measurements that are available in situ:

$$
\mathbf{R}_{\mathrm{v}}^{\mathrm{n}}(t) \mathbf{p}^{\mathrm{n}}(t)-\int \dot{\mathbf{R}}_{\mathrm{v}}^{\mathrm{n}}(\tau) \mathbf{p}^{\mathrm{n}}(\tau) d \tau+\text { const. }=\mathbf{R}_{\mathrm{v}}^{\mathrm{i}} \int \mathbf{u}^{\mathrm{i}}(\tau) d \tau
$$

This maps to the identifier (1.3):

$$
y=\mathbf{R} u
$$

with the input:

$$
u=\int \mathbf{u}^{\mathrm{i}}(\tau) d \tau
$$

the output:

$$
y=\mathbf{R}_{\mathrm{v}}^{\mathrm{n}}(t) \mathbf{p}^{\mathrm{n}}(t)-\int \dot{\mathbf{R}}_{\mathrm{v}}^{\mathrm{n}}(\tau) \mathbf{p}^{\mathrm{n}}(\tau) d \tau+\text { const } .
$$


In all laboratory and field experiments, the means of the input $u$ and output $y$ are shifted to zero so that only the rotation remains. This is the form Kinsey \& Whitcomb used in $[13,14]$ to identify the alignment between a FOG and DVL.

\subsubsection{Renavigation and performance metrics}

The goal of the DVL/FOG alignment problem is to improve DR navigation. To experimentally validate the rotor identifier, we compare DR trajectories calculated with the rotor alignment estimate to LBL observations. We will characterize the improvement in DR performance gained by using the rotor alignment estimate versus a visual alignment estimate. We will also compare to the alignment methods in $[13,14]$. All of this analysis is done in post-processing by renavigating the same raw sensor data using each alignment estimate.

\section{Position residual}

First, we calculate the difference between the DR estimate and the LBL measurement of vehicle position-this is the position residual ${ }^{18}$ :

$$
\begin{gathered}
\tilde{\mathbf{p}}=\mathbf{p}_{\mathrm{DR}}^{\mathrm{n}}-\mathbf{p}_{\mathrm{LBL}}^{\mathrm{n}} \\
{\left[\begin{array}{c}
\tilde{x} \\
\tilde{y} \\
\tilde{z}
\end{array}\right]=\left[\begin{array}{l}
x_{\mathrm{DR}}^{\mathrm{n}}-x_{\mathrm{LBL}}^{\mathrm{n}} \\
y_{\mathrm{DR}}^{\mathrm{n}}-x_{\mathrm{LBL}}^{\mathrm{n}} \\
z_{\mathrm{DR}}^{\mathrm{n}}-z_{\mathrm{LBL}}^{\mathrm{n}}
\end{array}\right]}
\end{gathered}
$$

As an example, (Figure 1-9) shows the components of the position residual in laboratory experiment 070 . We will use several aspects of this position residual as performance metrics.

\section{Residual component distributions}

The components of the position residual (1.71b) are sorted into bins, and the distribution is plotted for the residual along each direction. The horizontal axis will show

\footnotetext{
${ }^{18}$ The tilde decoration here on $\tilde{\mathbf{p}}$ denotes the residual, not the reversion operation in GA.
} 

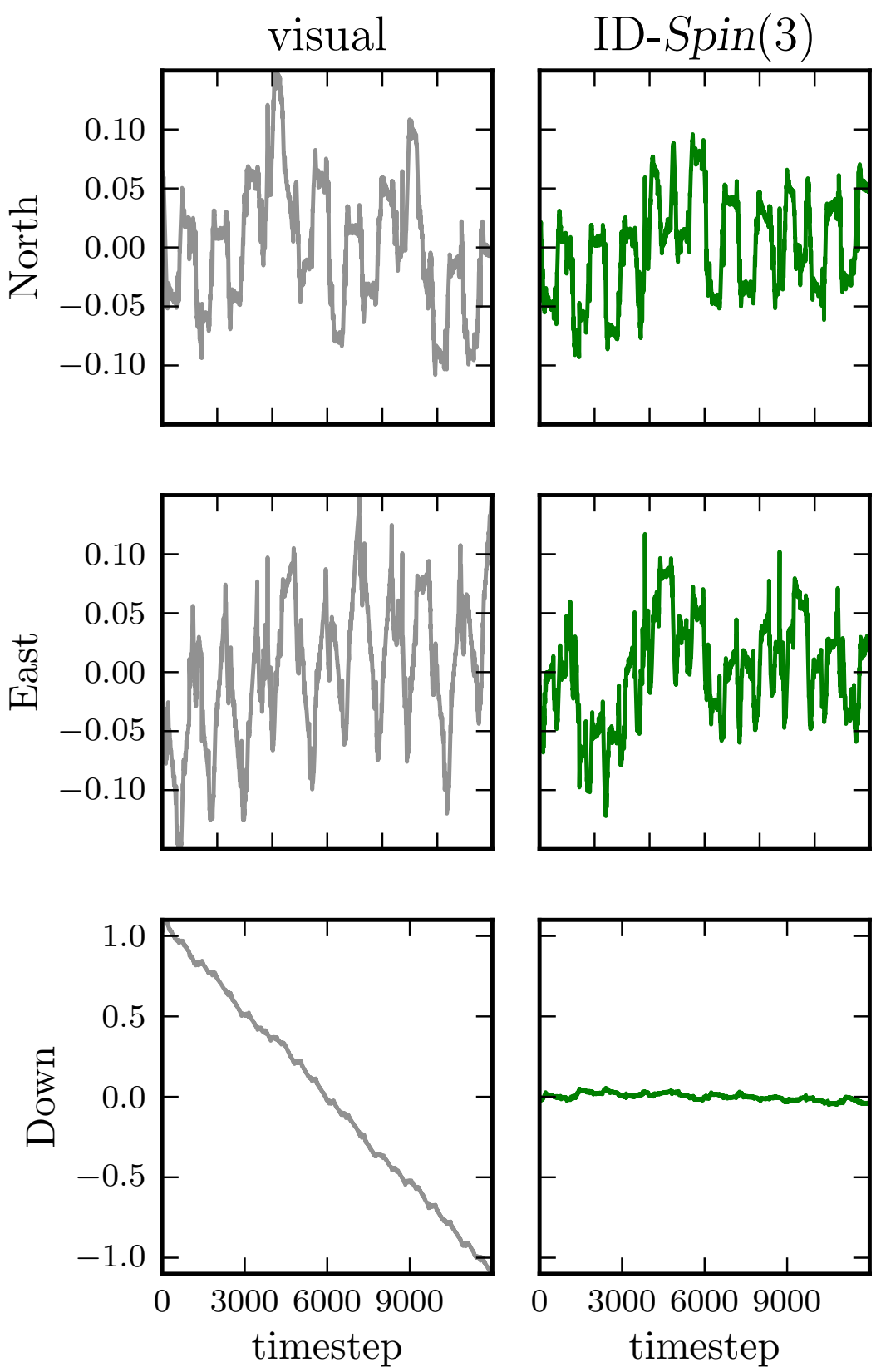

Figure 1-9: Components of the position residual from laboratory experiment 070 . In the left column, residuals from DR with the visual alignment estimate are plotted in gray. In the right column, residuals from DR with the ID-Spin(3) alignment estimate are plotted in green. Note that the vertical component of the residual from the visual alignment is much larger than the others, and that the ID-Spin(3) alignment reduces it considerably. 

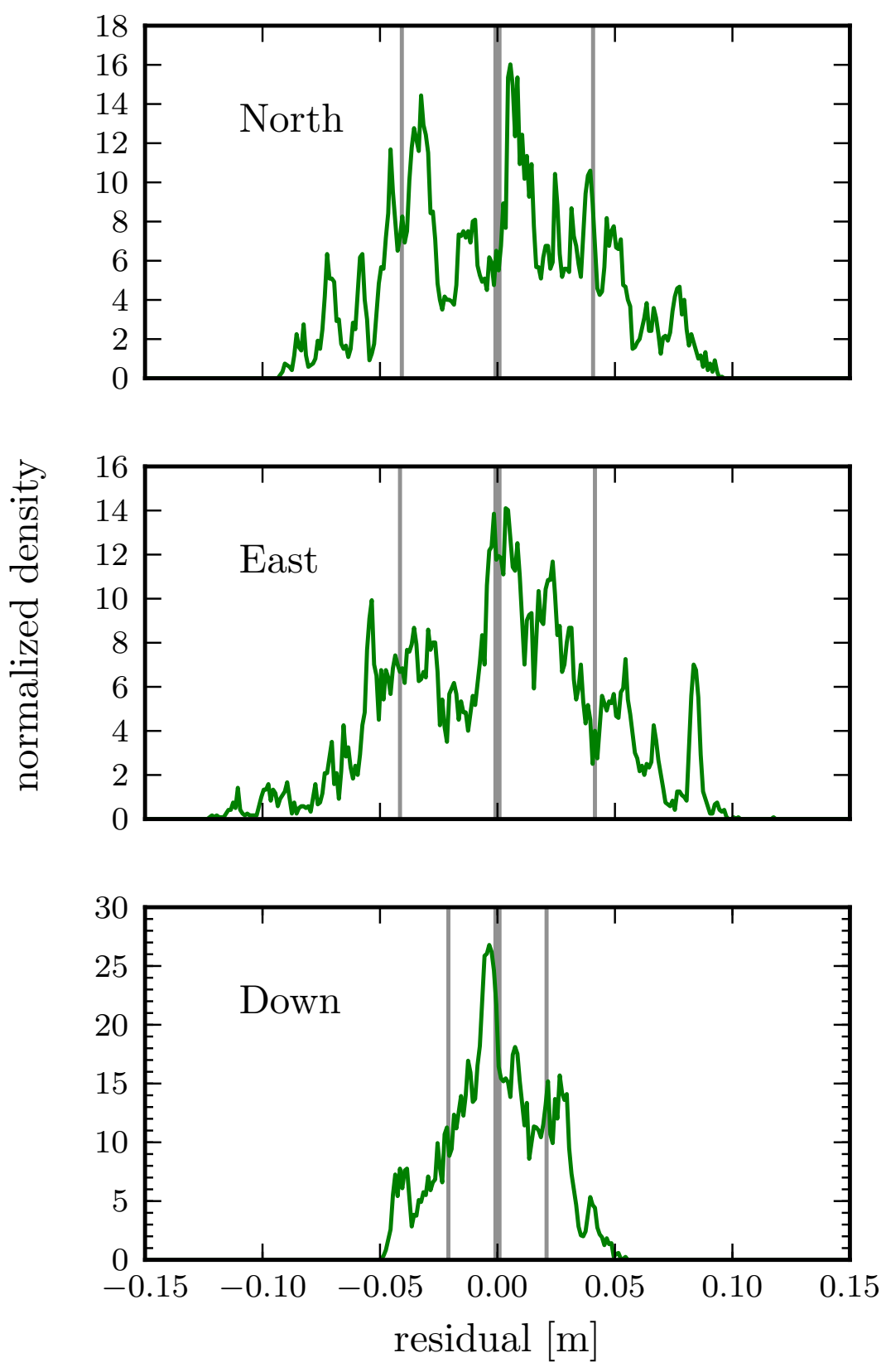

Figure 1-10: Distributions of the position residual components from laboratory experiment 070.

the residual, and the vertical axis will show the frequency-this is normalized so that it integrates to one. (Figure 1-10) 


\section{Residual component standard deviations}

To summarize the residual component distributions, we compute standard deviation of the residual in each direction. These are collected into the residual component standard deviation vector ${ }^{19}$ :

$$
\boldsymbol{\sigma}=\left[\begin{array}{c}
\sigma_{x} \\
\sigma_{y} \\
\sigma_{z}
\end{array}\right]=\left[\begin{array}{l}
\operatorname{std}\left(x_{\mathrm{DR}}^{\mathrm{n}}-x_{\mathrm{LBL}}^{\mathrm{n}}\right) \\
\operatorname{std}\left(y_{\mathrm{DR}}^{\mathrm{n}}-y_{\mathrm{LBL}}^{\mathrm{n}}\right) \\
\operatorname{std}\left(z_{\mathrm{DR}}^{\mathrm{n}}-z_{\mathrm{LBL}}^{\mathrm{n}}\right)
\end{array}\right]
$$

The Euclidean norm of this vector:

$$
\|\boldsymbol{\sigma}\|_{2}=\sqrt{\boldsymbol{\sigma} \boldsymbol{\sigma}^{T}}
$$

is termed the standard deviation vector norm ${ }^{20}$. This provides a convenient scalar performance metric that is suitable for computing ensemble statistics for cross-validation of each alignment estimate across the other datasets.

\section{Residual magnitude distribution}

Alternatively, we may consider the Euclidean norm of the position residual at each point in time:

$$
\|\tilde{\mathbf{p}}\|_{2}=\sqrt{\tilde{\mathbf{p}} \tilde{\mathbf{p}}^{T}}
$$

Since the magnitude of $\tilde{\mathbf{p}}$ is always positive, this produces a one-sided distribution (Figure 1-11). Again, the frequency axis is normalized so that the distribution integrates to one.

We will also study the integral of this distribution-the cumulative residual magnitude distribution. This provides another perspective on the residual-it shows the fraction of the residuals that fall below a certain magnitude. For example, Figure 1-12

\footnotetext{
${ }^{19}$ This is called the error vector in [13, 14,85$]$.

${ }^{20}$ This is called the error vector norm in $[13,14,85]$.
} 


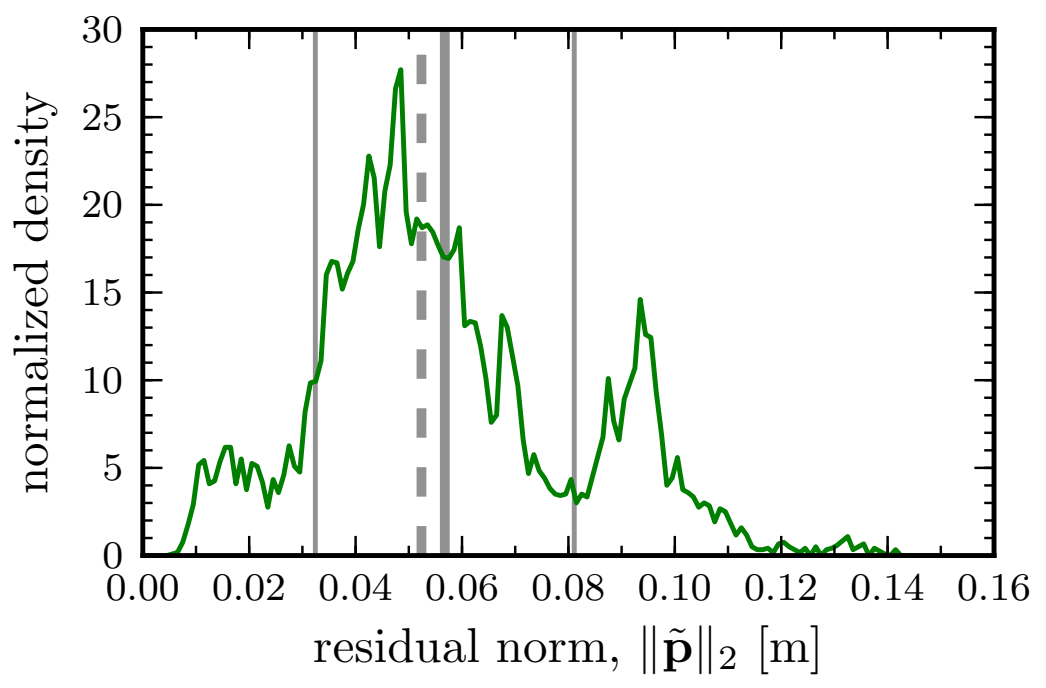

Figure 1-11: Distribution of the position residual magnitude from laboratory experiment 070 .

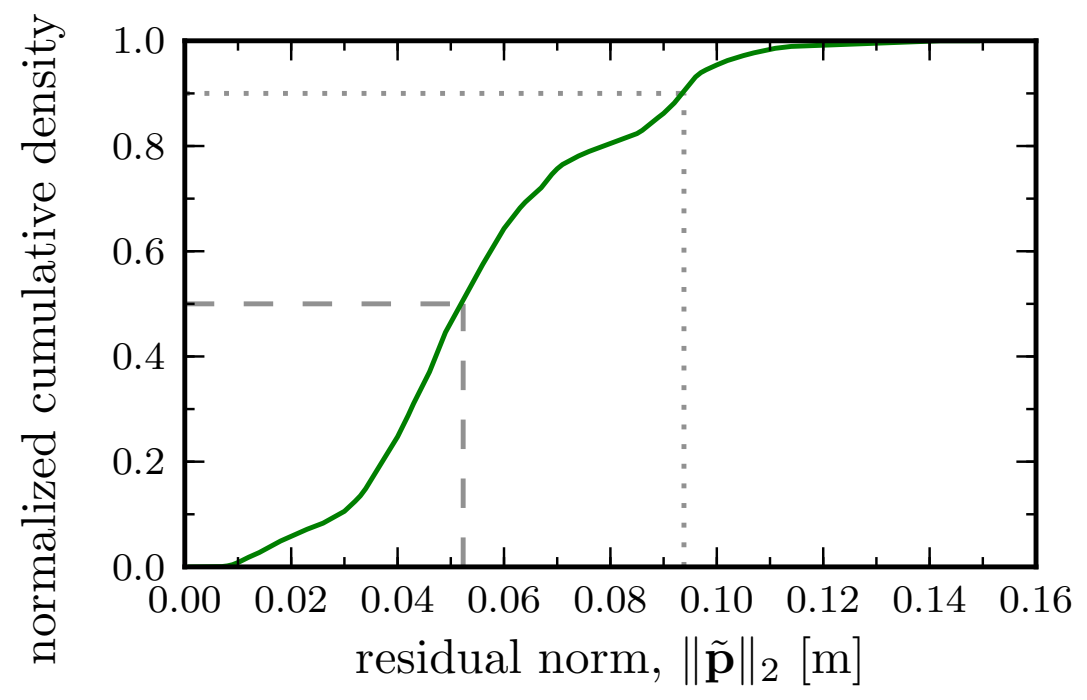

Figure 1-12: Cumulative distribution of the position residual magnitude from laboratory experiment 070 .

shows that half of the position residual vectors are less than 5.3 centimeters long, and $90 \%$ of them are less than 9.4 centimeters long.

\section{Mean and median residual magnitude}

Since the residual magnitude distribution is one-sided, we will use its mean and median to summarize it. For self-validation tests (renavigating the same dataset that 
was used to estimate the rotation) we will show standard deviations as well.

\section{Different alignment methods}

We will compare DR trajectories using four alignment methods:

visual This is the initial alignment estimate with beam 3 of the DVL pointing down and toward the forward starboard quarter. The alignment mark on the DVL housing points straight ahead-the DVL coordinate frame is misaligned from the FOG coordinate frame by $45^{\circ}(\pi / 2 \mathrm{rad})$ in heading and $0^{\circ}$ in roll and pitch.

LS-SO(3) The batch method from [58] to estimate the rotation matrix using LS constrained to $S O(3)$ as applied to DVL/FOG alignment in $[13,55]$.

ID-SO(3) The adaptive identifier on $S O(3)$ from [14,61]. This has been slighly modified by normalizing the input $u$ and output $y$ fed to the identifier. This change preserves directional information in the measurements, but changes the behavior of the identifier with respect to gains. It was necessary for the identifier to work on field datasets from Sentry.

ID-Spin(3) The adaptive identifier on $\operatorname{Spin}(3)$, the group of rotors in $\mathbb{G}_{3}$. This is the method detailed in [15] and earlier in this chapter.

\subsection{Laboratory Experiments}

This section evaluates the performance of the rotor identifier on the FOG/DVL alignment problem using data from controlled experiments with a laboratory ROV. This data is from the same series of six experiments used in $[13,14,85]$, and results from the rotor identifier are compared to those from the CLS and $S O(3)$ alignment methods reported there.

Section 1.5.1 briefly reviews the experimental setup and conditions ${ }^{21}$ given in $[13$, 14,85]. The rotor identification results and renavigated trajectories from each exper-

\footnotetext{
${ }^{21}$ By necessity, much of this is paraphrased from $[13,14,85]$; it is reviewed here for completeness.
} 


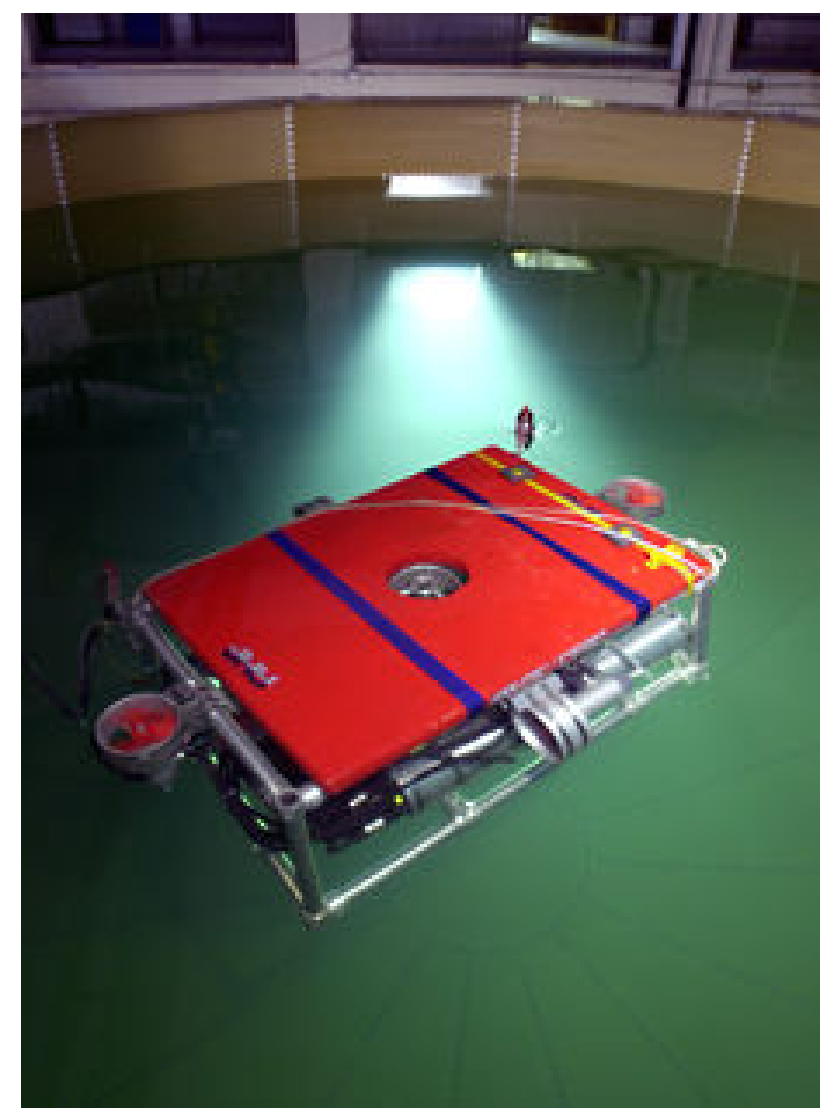

Figure 1-13: The Johns Hopkins University ROV is a laboratory system outfitted with sensors similar to those typically found on oceanographic research vehicles. It is a testbed vehicle used for for dynamics, control, and navigation studies for deep submergence vehicles. (photo credit: JHU DSCL)

iment are given in section 1.5.2, and alignment cross-validation between the different experiments is discussed in section 1.5.3.

\subsubsection{JHU Hydrodynamics Test Facility and JHUROV}

Data in these experiments was collected in the Hydrodynamics Test Facility at the Johns Hopkins University (JHU). JHUROV (Figure 1-13) is a testbed vehicle for dynamics, control, and navigation studies for deep submergence vehicles. It is instrumented with sensors similar to those found on vehicles in the field, except that some of the laboratory systems have shorter range and higher precision. The sensors relevant to this study are listed in Table 1.2.

The pressure depth transducer (Paroscientific Digiquartz) is precise to 10 parts 
Table 1.2: Navigation sensors on JHUROV used in laboratory experiments. (adapted from $[13,14,85])$

\begin{tabular}{|c|c|c|c|c|}
\hline quantity & sensor & sample rate & precision & range \\
\hline$(x, y)$ position & $\begin{array}{c}300 \mathrm{kHz} \text { LBL } \\
\text { (Marquest) SHARPS }\end{array}$ & $5 \mathrm{~Hz}$ & $5 \mathrm{e}^{-3} \mathrm{~m}$ & $100 \mathrm{~m}$ \\
\hline depth & $\begin{array}{c}\text { pressure transducer } \\
\text { (Paroscientific Digiquartz) }\end{array}$ & $7 \mathrm{~Hz}$ & $\mathrm{~m}$ & $10 \mathrm{~m}$ \\
\hline orientation & $\begin{array}{l}\text { fiber-optic gyroscope (FOG) } \\
\text { (IXSEA PHINS) }\end{array}$ & $10 \mathrm{~Hz}$ & $\begin{array}{c}0.01^{\circ} \\
1.7 \mathrm{e}^{-4} \mathrm{rad}\end{array}$ & $\begin{array}{l}360^{\circ} \\
2 \pi \mathrm{rad}\end{array}$ \\
\hline velocity & $\begin{array}{l}1200 \mathrm{kHz} \text { DVL } \\
\text { (TRDI WHN) }\end{array}$ & $10 \mathrm{~Hz}$ & $0.3 \%$ & $\sim 30 \mathrm{~m}$ \\
\hline
\end{tabular}

per million over a depth of 10 meters at $7 \mathrm{~Hz}$. A two-beacon $300 \mathrm{kHz}$ LBL system (Marquest) provides $(x, y)$ position measurements at rates up to $18 \mathrm{~Hz}$. Combined with the depth constraint, the position is accurate to within $7 \mathrm{~mm}$ at one standard deviation [85]. Velocity over ground is measured by a $1200 \mathrm{kHz}$ DVL (Teledyne RD Instruments Workhorse Navigator) with precision ${ }^{22}$ better than $0.3 \%$ at $5-10 \mathrm{~Hz}$. Vehicle heading is measured within $0.05^{\circ}$ and roll \& pitch within $0.01^{\circ}$ at a rate of 10 $\mathrm{Hz}$ by a FOG (Ixsea PHINS). In these experiments, the FOG did not record angular rates, so they are obtained by numerical differentiation [85].

More details on the Hydrodynamics Test Facility and JHUROV can be found in $[85,86]$.

\subsubsection{Alignment identification and renavigated trajectory}

The rotor identifier was used to estimate DVL/FOG alignment on JHUROV on each of six laboratory experiments. The closed-loop trajectories were each 40 minutes long to collect enough data for the LS estimator while avoiding potential problems from unmodeled DR integration drift [85]. Data from expt070 was used to introduce the renavigation error metrics in section 1.4.2.

Figure 1-14 shows the normalized distribution of the residual magnitude. The DR track using the visial alignment estimate disagrees with the LBL observations by more than one meter in some places. The alignment estimated by the rotor identifier brings

\footnotetext{
${ }^{22}$ standard deviation of error in velocity component measured along a single acoustic beam
} 

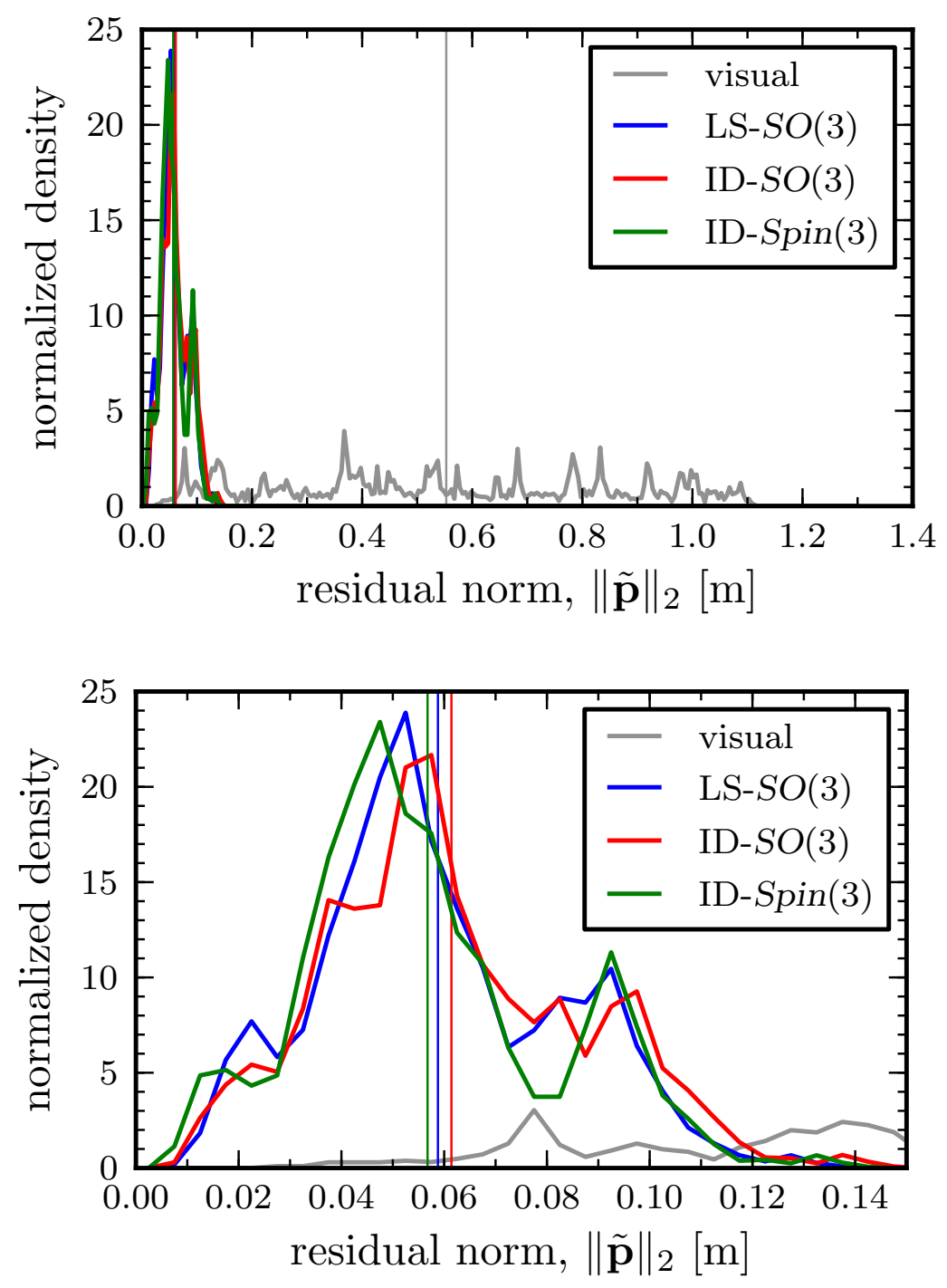

Figure 1-14: Residual magnitude distribution or JHUROV laboratory dataset 070, renavigated using different DVL/FOG alignment estimates. The lower figure is a zoom into the left side of the upper figure, to see the small differences between the alignment methods. Vertical lines indicate the mean of the residual magnitude. Note that the rotor identifier presented here performs comparably to the LS-SO(3) batch method in [13] and the ID-SO(3) method with normalized inputs, adapted from [14]. All three methods perform significantly better than the visual alignment estimate. 

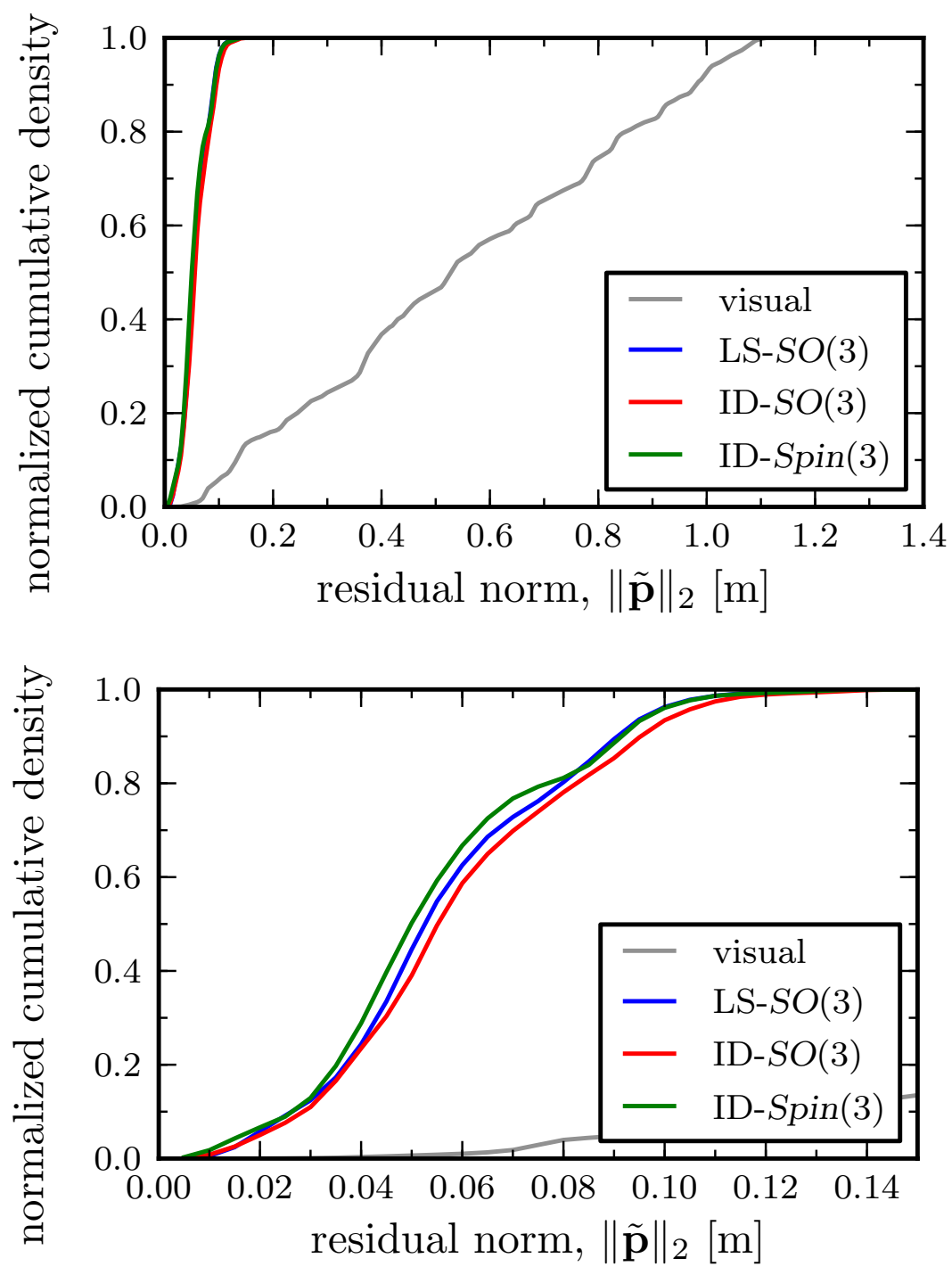

Figure 1-15: Cumulative residual magnitude distribution for JHUROV laboratory dataset 070, renavigated using different DVL/FOG alignment estimates. The lower figure is a zoom into the left side of the upper figure, to see the small differences between the alignment methods. Note that the rotor identifier presented here performs comparably to the LS-SO(3) batch method in [13] and the ID-SO(3) method with normalized inputs, adapted from [14]. All three methods perform significantly better than the visual alignment estimate.

all residuals within fourteen centimeters, and the rotor identifier performs comparably to LS-SO(3) and ID-SO(3) methods.

Figure 1-15 shows the normalized cumulative distribution of the residual magnitude. This plot makes it easy to determine what fraction of the residuals fall below a particular level. For example, $50 \%$ of the residuals in expt070 fall below $5.3 \mathrm{~cm}$ when 
DR uses the alignment estimate from the rotor identifier, but that same fraction of residuals extends as high as $53 \mathrm{~cm}$ magnitude using the visual alignment estimate.

The other laboratory datasets show similar results. When using the alignment from the rotor identifier:

- The residual component distributions are narrower, closer to zero from both sides.

- The one-sided residual magnitude distribution is weighted to the left, in the range of centimeters rather than tens of centimeters.

- The residual magnitude cumulative distribution becomes much steeper and moves toward the upper left corner, showing again that the residuals are much smaller.

The effect of using the ID- $S O(3)$ or ID- $S O(3)$ alignment estimate is similar-the rotor identifier performs comparably to these previous results.

Table 1.3 shows the summarized statistics of the renavigation residuals for all six laboratory experiments. In this data, the residuals in depth are the largest starting with the visual alignment estimate, and using the alignment estimated by the rotor identifier reduces them considerably. Using the alignment estimated by the rotor identifier reduces the standard deviation vector norm to $10-15 \%$ of its original value with the visual alignment estimate. The mean and median of the magnitude of the residuals are similarly reduced. Figure 1-16 shows these quantities graphically. Again, the rotor identifier performs comparably to the previously reported rotation matrix methods, and all of these perform better than the original visual alignment method. Of course, the performance improvement will always depend on how close the visual alignment estimate is in the first place.

\subsubsection{Cross-validation}

It is reasonable to ask how the alignment estimated using one dataset performs on another. If the sensors are not moved between experiments, the estimated alignment 


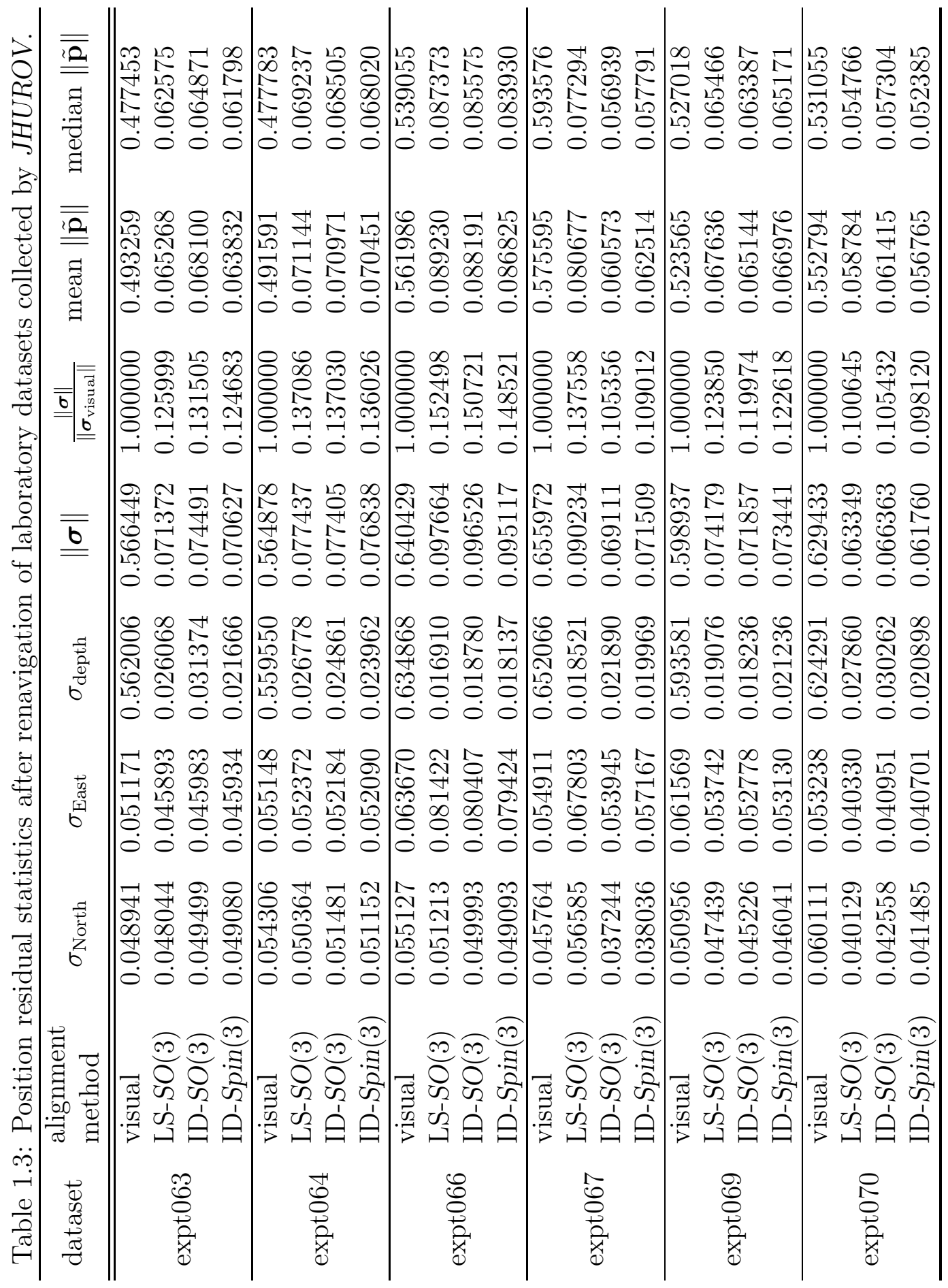



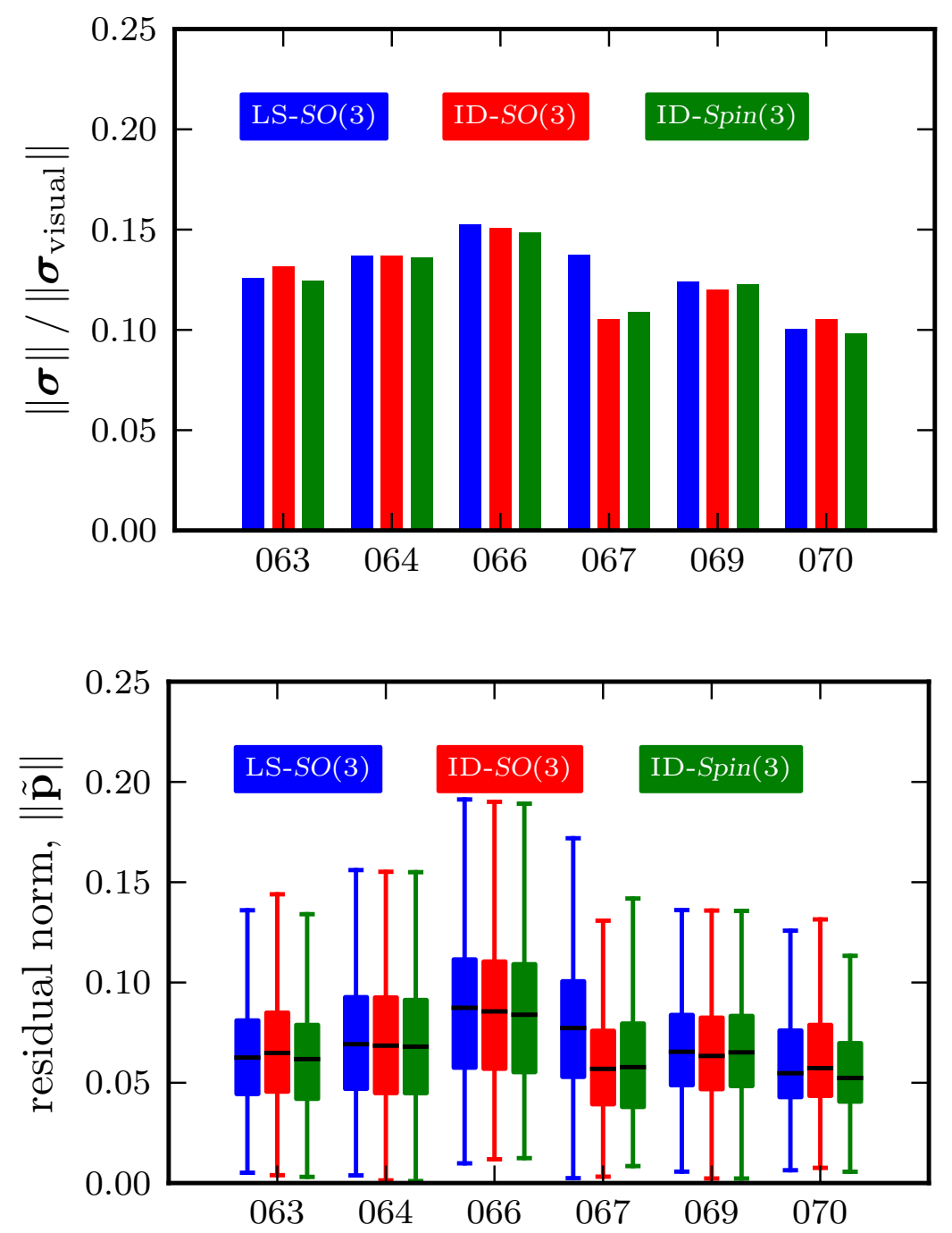

Figure 1-16: Self-validation of alignment estimates for each laboratory dataset. Barplot shows the normalized standard deviation vector norm as defined in (1.73) and represent the improvement in navigation over the original (visual) alignment estimate. Boxplot shows the residual norm for each experiment. 
should perform comparably on other trajectories. Figure 1-17 shows the normalized standard deviation vector magnitude using the alignment estimated from each laboratory dataset to renavigate the trajectory of each other dataset for each of the three different alignment methods. The plots show fairly uniform levels with some lower magnitudes along the diagonal. This should be expected, and indicates that the best renavigation performance is on the same dataset used for the estimate. Figure 1-18 collects this data into ensembles and displays the statistics for each alignment estimate renavigating every other dataset. The normalized standard deviation vector magnitude is comparable across alignment estimates-this indicates good cross-validation of the estimates. Again, the rotor identifier performs comparably to the previously reported rotation matrix methods.

\subsubsection{A note on transient response}

Unlike batch methods for estimating a rotation, the adaptive identifiers are dynamic systems. It can be important to consider the transient response of such systems, especially when they will be subject to unknown excitation (e.g. measurement noise). Figure 1-19 compares the time history of the ID-SO(3) and ID-Spin(3) identifiers on expt070, using a scalar (geodesic) residual with the estimate from the batch LS-SO(3) method as a reference. It is important to note that this is experimental data without a ground truth, so the LS-SO(3) may not be more correct than either of the others.

Both adaptive identifiers initially converge toward the same rotation estimate as the LS-SO(3) method, within about $10^{-3}$ radians. The interesting event to note is that the identifier estimates fluctuate between input/output vector pairs 4000 and 8000. The magnitude of the input $u$ and output $y$ is initially large, and both pass through zero near vector pair 6000, before increasing in magnitude again. This is because the means of $u$ and $y$ were set to zero when mapping the DR measurements into the form of the identifier (Section 1.4.1). As $u$ and $y$ pass through zero, any noise in the measurements becomes proportionally larger, and the identifiers will react to that noise.

This problem can be handled pragmatically in this application by averaging the 
LS- $S O(3)$

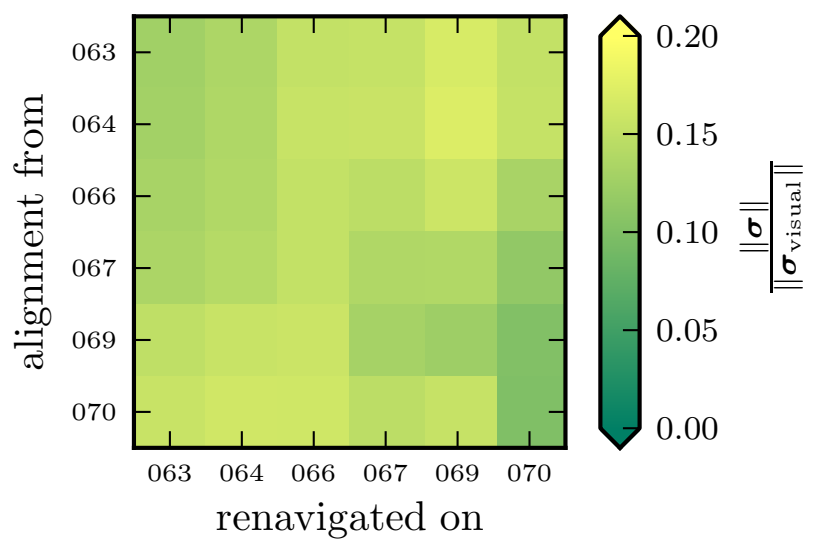

ID- $S O(3)$
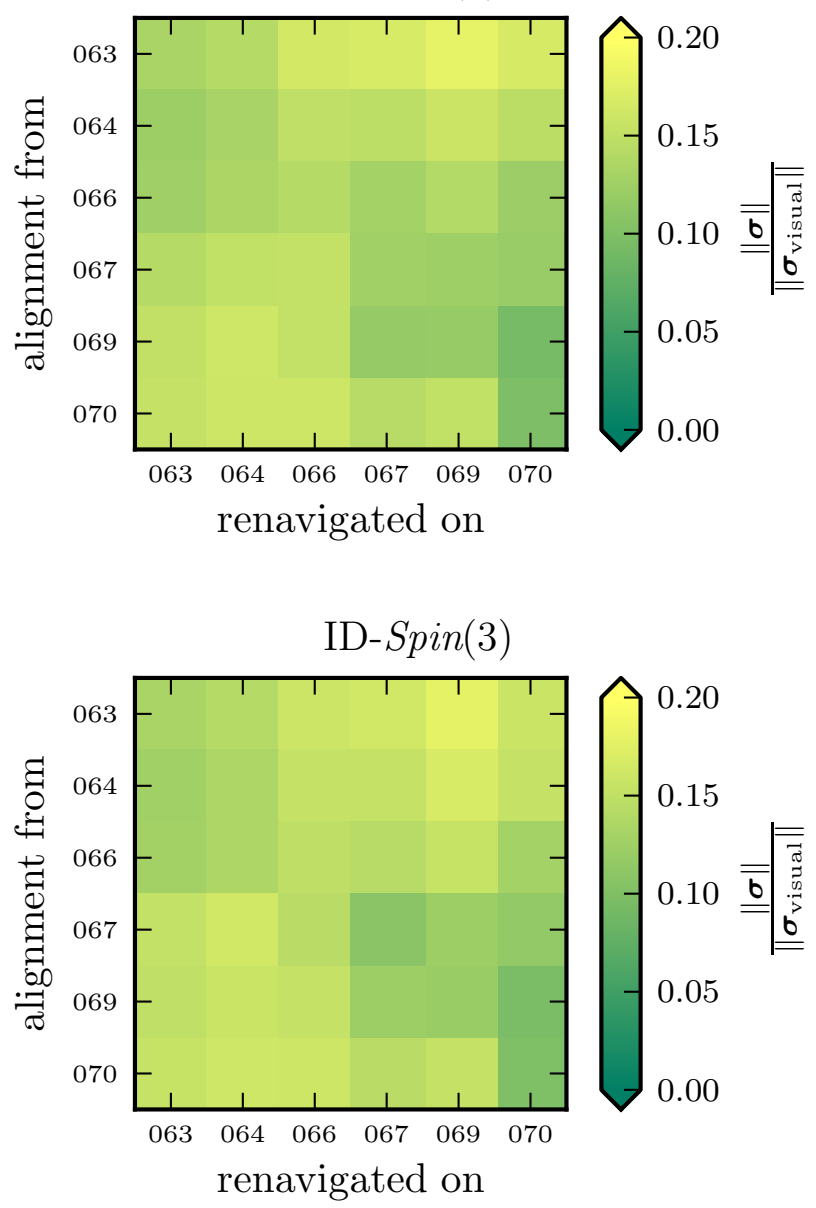

Figure 1-17: Cross-validation of alignment estimates on each other laboratory dataset. Colors indicate the magnitude of the normalized standard deviation vector. 

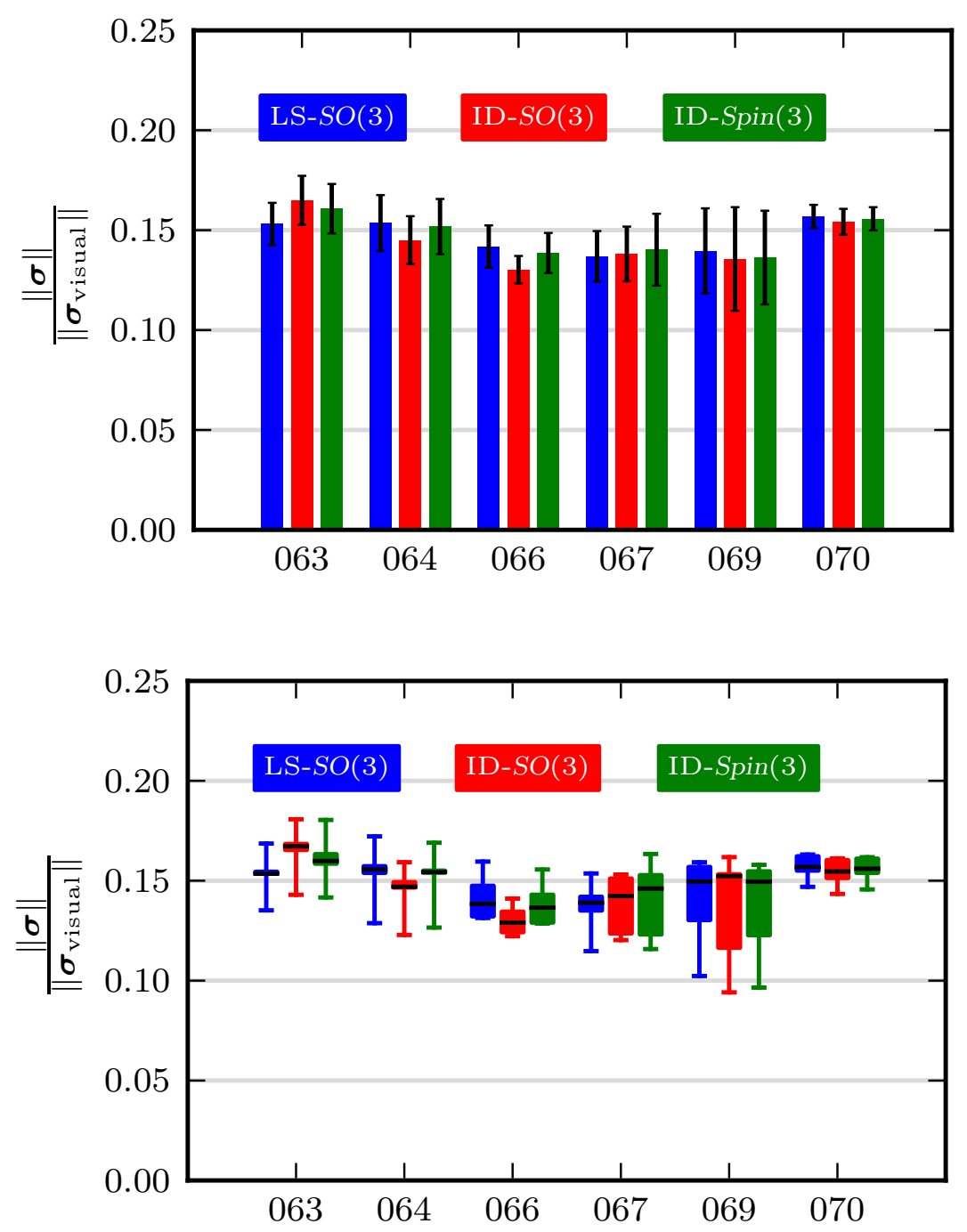

Figure 1-18: Cross-validation statistics of alignment estimates over all laboratory datasets. The alignment estimate from one dataset was used to renavigate the data from all other datasets. Bars show the mean normalized magnitude of the standard deviation vector, and whiskers indicate the standard deviation of this magnitude across the other datasets. Boxplots show the median, first quartile, and total range of the normalized magnitude of the standard deviation vector. 


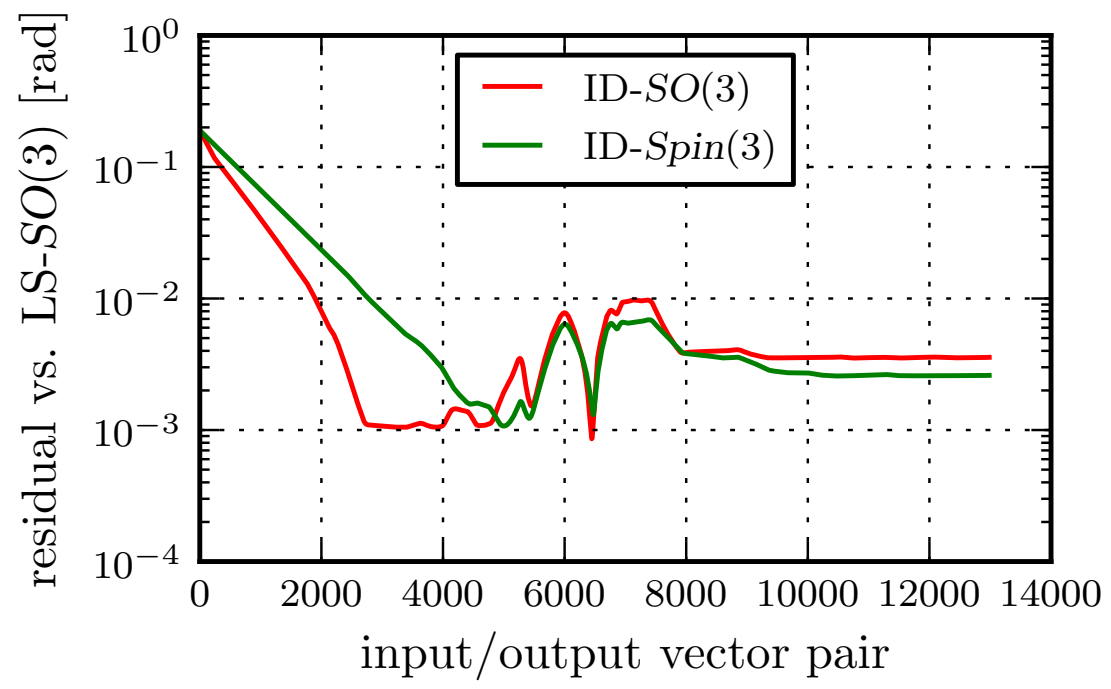

Figure 1-19: Scalar residual with respect to alignment estimated by LS-SO(3) method.

estimated rotor $^{23}$ over several timesteps. This particular behavior may not be important in other applications of rotation identification-it depends on how the inputs and outputs vary. Nevertheless, it raises an interesting question: what modifications might enable the rotor identifier to reject noise better? One approach might involve adding some damping to the system-using both proportional and derivative gains in the output error feedback regulator. This seems like an obvious next step, but keep in mind that is due mostly to the clarity offered by the GA formulation-this behavior of the transient response was effectively illustrated with a scalar residual, and the simple interpretation of the identifier as a regulator was enabled by working in rotors and bivectors.

\subsection{Field Experiments}

This section evaluates the performance of the rotor identifier on the FOG/DVL alignment problem using data from field deployments of the oceanographic AUV Sentry. Results from the rotor identifier are compared to those from the CLS and $S O(3)$ alignment methods reported in $[13,14]$.

\footnotetext{
${ }^{23}$ This is another area where the GA formulation is useful-it is much easier to average rotors than rotation matrices (see, e.g. [64,65]).
} 
Table 1.4: Navigation sensors on Sentry used in field experiments.

\begin{tabular}{|c|c|c|c|c|}
\hline quantity & sensor & sample rate & precision & range \\
\hline$(x, y)$ position & $\begin{array}{c}12 \mathrm{kHz} \text { LBL } \\
\text { (WHOI) }\end{array}$ & $0.1 \mathrm{~Hz}$ & $\sim 0.25 \mathrm{~m}$ & $>10000 \mathrm{~m}$ \\
\hline depth & $\begin{array}{c}\text { pressure transducer } \\
\text { (Paroscientific Digiquartz) }\end{array}$ & $7 \mathrm{~Hz}$ & $0.02 \mathrm{~m}$ & $6500 \mathrm{~m}$ \\
\hline orientation & $\begin{array}{c}\text { fiber-optic gyroscope (FOG) } \\
\text { (IXSEA PHINS) }\end{array}$ & $10 \mathrm{~Hz}$ & $\begin{array}{c}0.01^{\circ} \\
1.7 \mathrm{e}^{-4} \mathrm{rad}\end{array}$ & $\begin{array}{c}360^{\circ} \\
2 \pi \mathrm{rad}\end{array}$ \\
\hline velocity & $\begin{array}{l}300 \mathrm{kHz} \text { DVL } \\
(\mathrm{TRDI} \text { WHN) }\end{array}$ & $2 \mathrm{~Hz}$ & $0.3 \%$ & $\sim 200 \mathrm{~m}$ \\
\hline
\end{tabular}

Section 1.6.1 briefly reviews Sentry and its navigation sensors. The rotor identification results and renavigated trajectories from each experiment are given in section 1.6.2, and alignment cross-validation between the different experiments is discussed in section 1.6.3.

\subsubsection{Sentry field deployments on the Håkon Mosby mud volcano}

The rotor identifier was applied to navigational data from Sentry field deployments on the Håkon Mosby mud volcano in the Barents Sea. These dives were part of a research cruise led by Dr. Antje Boetius with the goal of characterizing the bathymetry, subbottom composition, and chemistry surrounding the mud volcano. The Sentry team deployed and surveyed a LBL net of three transponders for $\operatorname{six}^{24}$ dives on the same site.

A brief overview of the AUV Sentry (Figure 1-20) is given in section 0.2.5. Specific to the problem in this section, we will be using navigational data from the sensors listed in Table 1.4, comparing DR vehicle trajectories to LBL observations. 


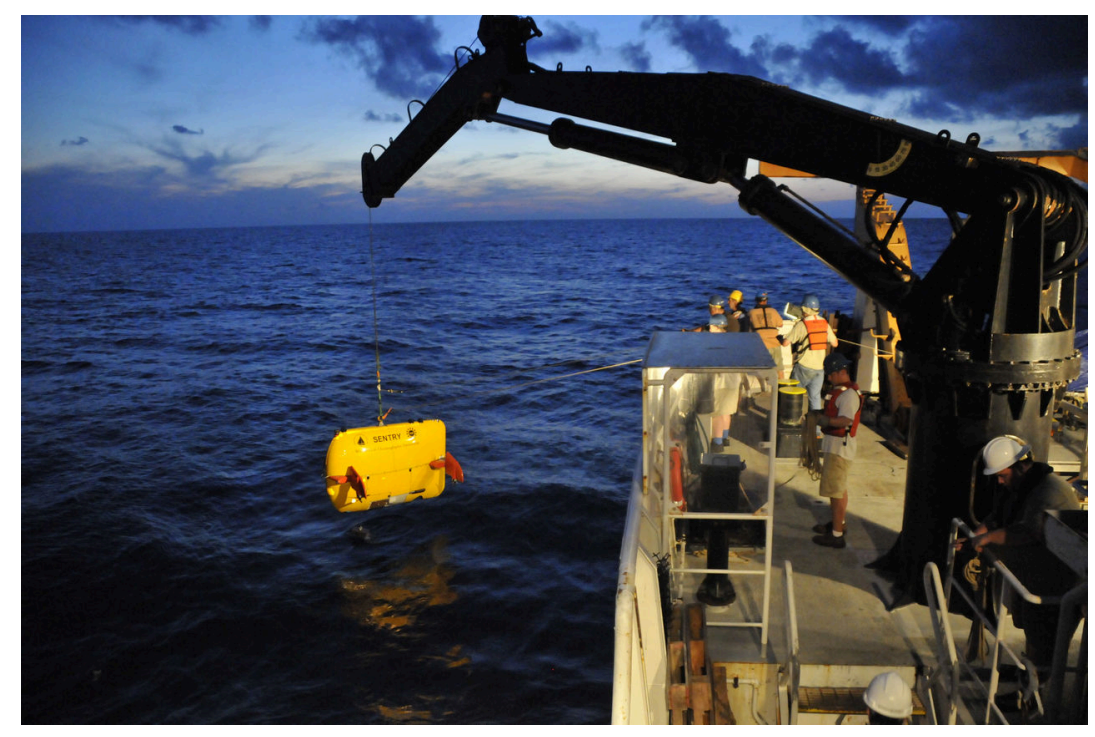

Figure 1-20: The NDSF AUV Sentry is an unmanned oceanographic research vehicle that dives as deep as 6500 meters to perform various scientific missions. Sentry navigates primarily by DR with a FOG and DVL. The FOG is located in the main pressure housing, roughly amidships and just below the name label. The DVL is on the keel just aft from the bow. The large separation between these sensors on a vehicle that may deform under extreme pressure (even slightly), motivates our study of stable, in-situ sensor alignment methods. (photo credit: D Yoerger, ABE/Sentry Group, WHOI)

\subsubsection{Alignment identification and renavigated trajectory}

Figure 1-21 shows the normalized distribution of each component of the residual, and

Figure 1-22 shows the normalized distribution of the residual magnitude for Sentry075. The magnitude distribution of the residuals is shifted slightly left (i.e., smaller) when using the alignment from the rotor identifier vs. the visual estimate. This is also reflected in the mean of the distribution. Again, the rotor identifier performs comparably to the previously reported rotation matrix methods.

Figure 1-23 shows the normalized cumulative distribution of the residual magnitude. This plot makes it easy to determine what fraction of the residuals fall below a particular level. For example, 50\% of the residuals in Sentry075 fall below $3.3 \mathrm{~m}$ when DR uses the alignment estimate from the rotor identifier, but that same fraction

\footnotetext{
${ }^{24}$ The last dive of the cruise, Sentry078, was terminated early due to an actuator failure and imminent weather. Data from that dive is not used in this section.
} 

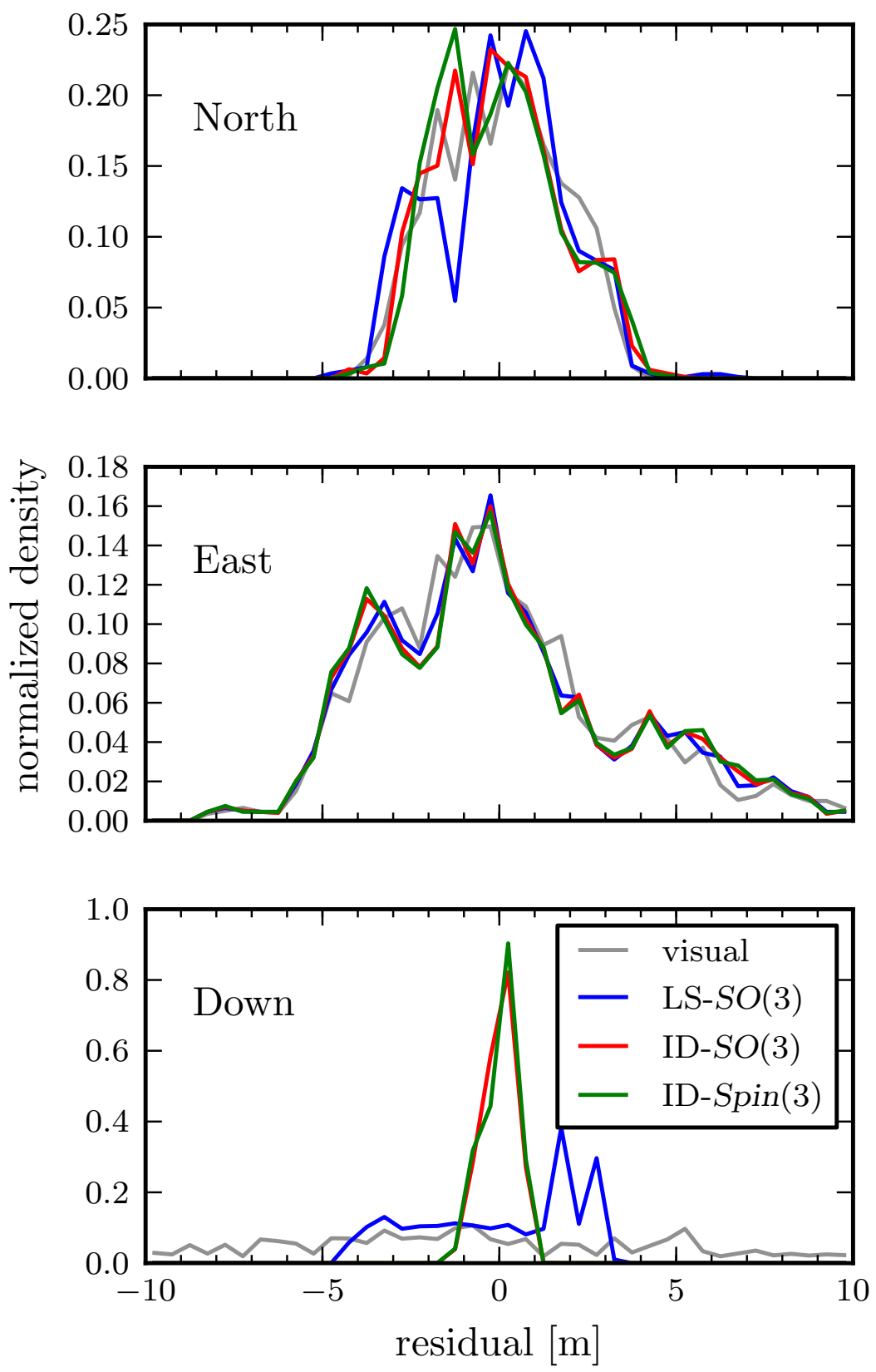

Figure 1-21: Distributions of residual components for Sentry075, renavigated using different DVL/FOG alignment estimates. Note that the rotor identifier presented here performs comparably to the LS-SO(3) batch method in [13] and the ID- $S O(3)$ method with normalized inputs, adapted from [14]. 


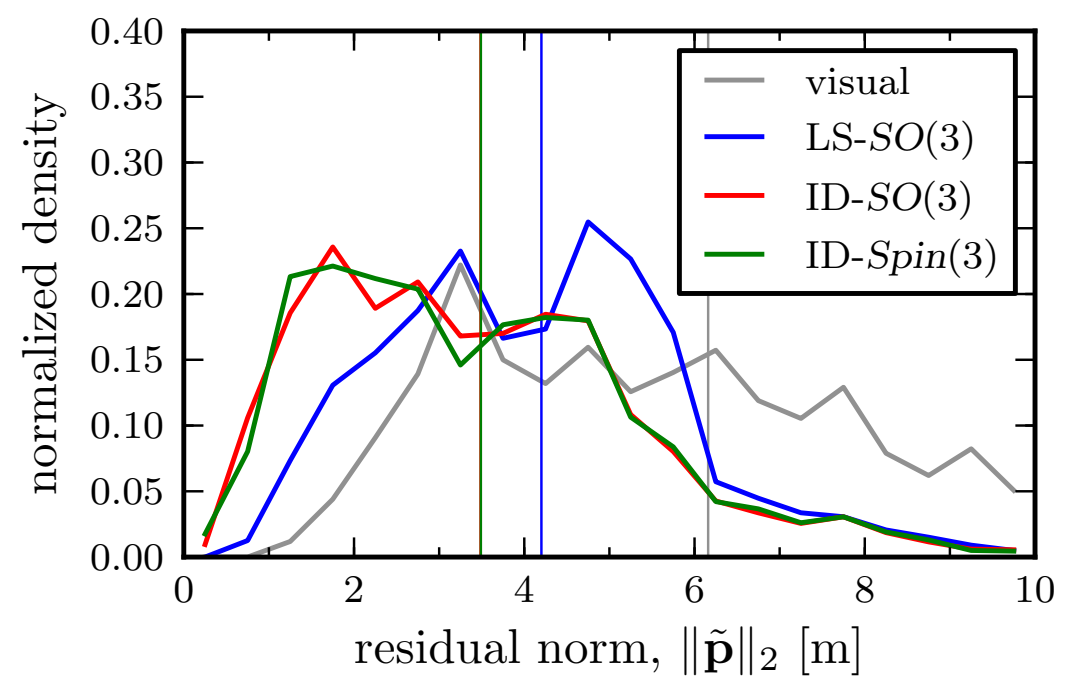

Figure 1-22: Residual magnitude distribution for Sentry075, renavigated using different DVL/FOG alignment estimates. Vertical lines indicate the mean of the residual magnitude. Note that the rotor identifier presented here performs comparably to the LS- $S O(3)$ batch method in [13] and the ID- $S O(3)$ method with normalized inputs, adapted from [14].

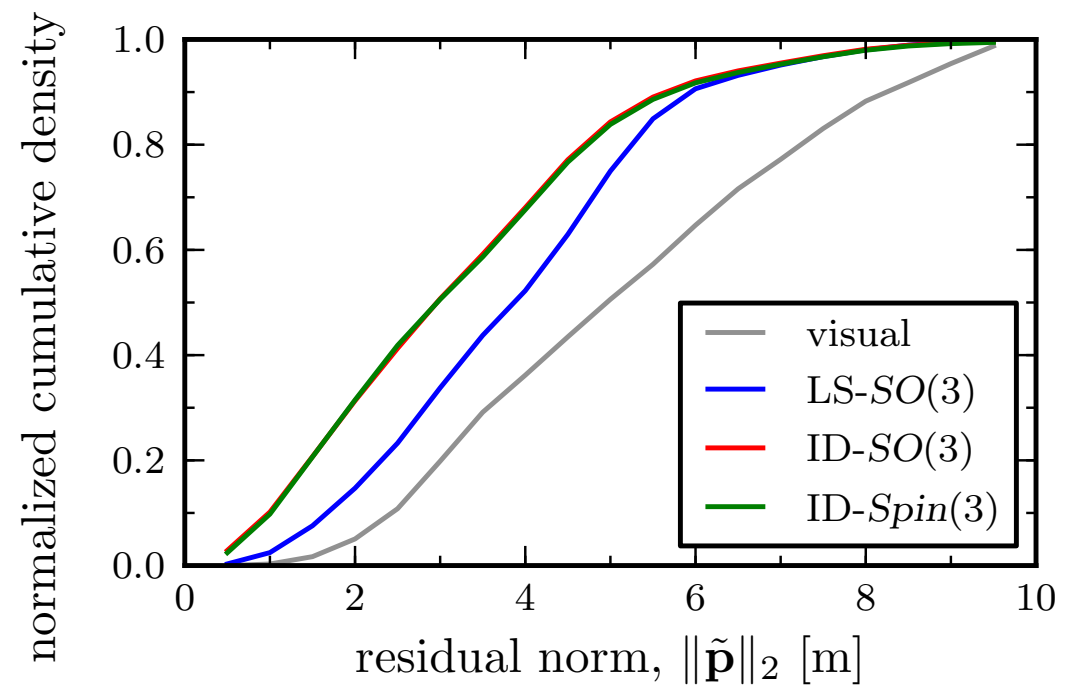

Figure 1-23: Cumulative residual magnitude distribution for Sentry075, renavigated using different DVL/FOG alignment estimates. Note that the rotor identifier presented here performs comparably to the LS-SO(3) batch method in [13] and the ID- $S O(3)$ method with normalized inputs, adapted from [14]. 
of residuals extends to $5.7 \mathrm{~m}$ magnitude using the visual alignment estimate. This represents a modest improvement, and indicates that the initial visual estimate was very close.

Table 1.5 shows the summarized statistics of the renavigation residuals for all five field experiments ${ }^{25}$. Figure 1-24 shows these quantities graphically. Again, the rotor identifier performs comparably to the previously reported rotation matrix methods. For some dives, these perform only slightly better than the original visual alignment method. This indicates that the visual alignment estimate was very close to begin with, and finer alignment is difficult to observe in these trajectories.

\subsubsection{Cross-validation}

Again, it is reasonable to ask how the alignment estimated using one dataset performs on another. Figure 1-17 shows the normalized standard deviation vector magnitude using the alignment estimated from each laboratory dataset to renavigate the trajectory of each other dataset for each of the three different alignment methods. The alignment estimated on dive 073 produces decent navigation across all other dives except for 075. This may be an artifact of the specific trajectories, but warrants future attention. The alignment estimates from Sentry075 perform well across all other dives. The estimates from dive 076 , on the other hand, do not do well in cross validation. Sentry came in contact with the seafloor several times during low altitude operations in dive 076 . The DR data from that dive is suspect, but still included here for completeness.

Figure 1-26 collects this data into ensembles and displays the statistics for each alignment estimate renavigating every other dataset. The normalized standard deviation vector magnitude varies across alignment estimates-staying close to one for the most part. The alignment estimates from dive 076 still perform poorly, probably due to low altitude and contact with the bottom. The statistics from dive 073 also show that the identified estimates tend to do worse than the visual estimate across

\footnotetext{
${ }^{25}$ Sentry came in contact with the seafloor several times during low altitude operations in dive 076. The DR data from that dive is suspect, but still included here for completeness.
} 


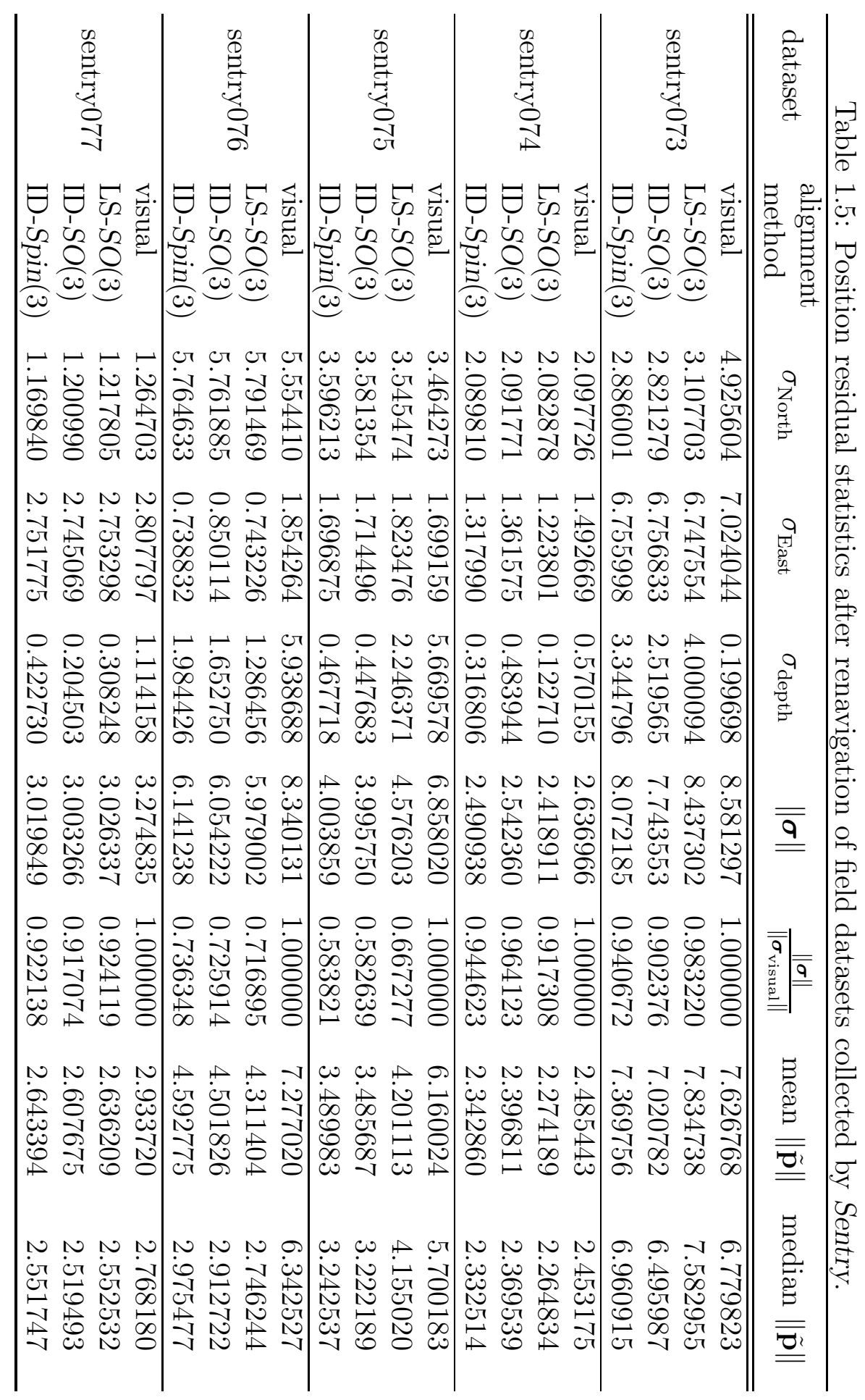



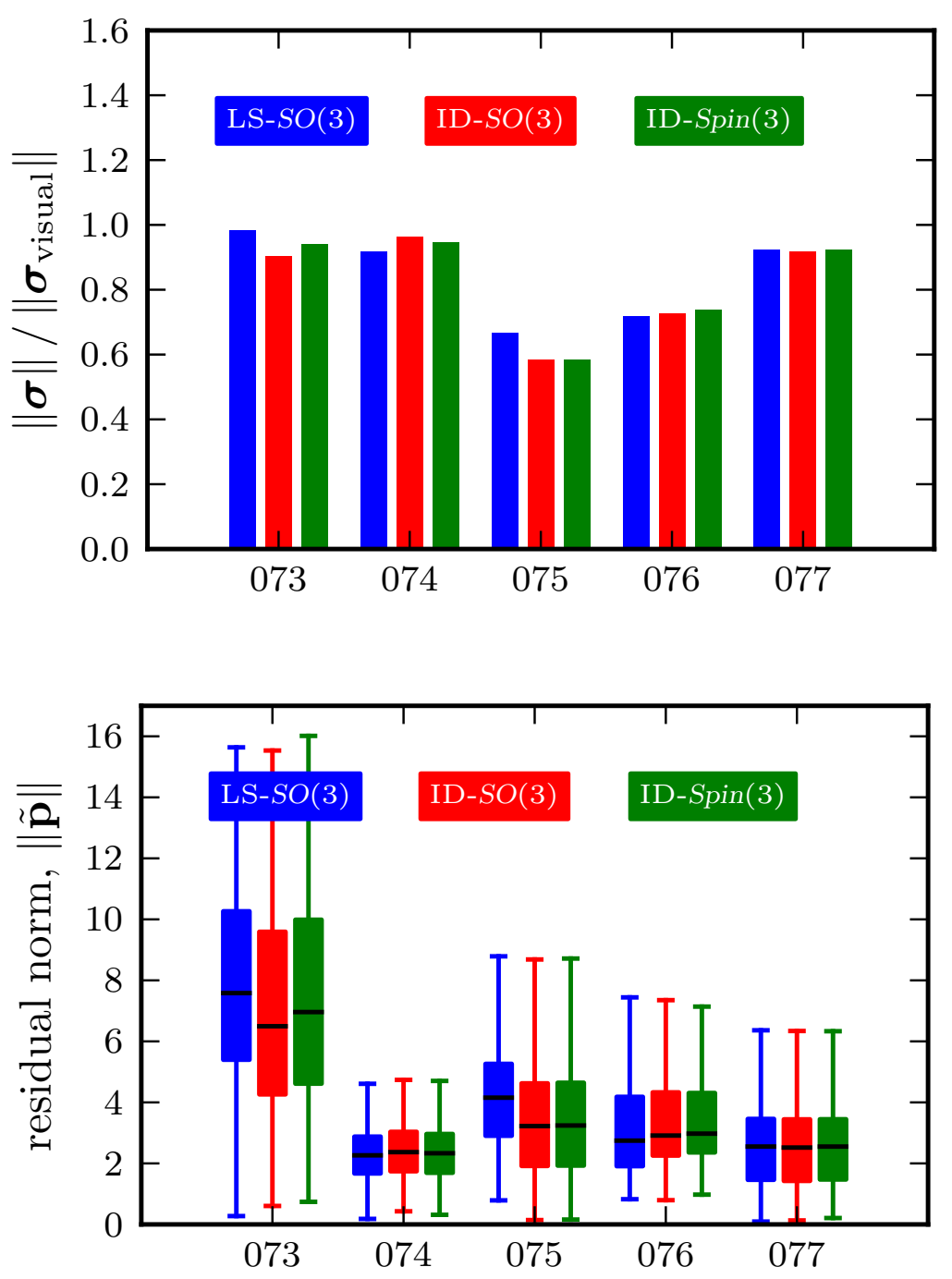

Figure 1-24: Self-validation of alignment estimates for each field dataset.

the other datasets. Sentry 073 covered more distance at higher altitude, with little change in depth-a trajectory that may not have provided enough information for any of the identifiers. Again, the rotor identifier performs comparably to the previously reported rotation matrix methods.

\subsection{Discussion and Conclusions}

This chapter has presented a novel alternative approach to the sensor alignment problem. Rotations are encoded using rotors in Geometric Algebra, so that algebraic 
LS- $S O(3)$
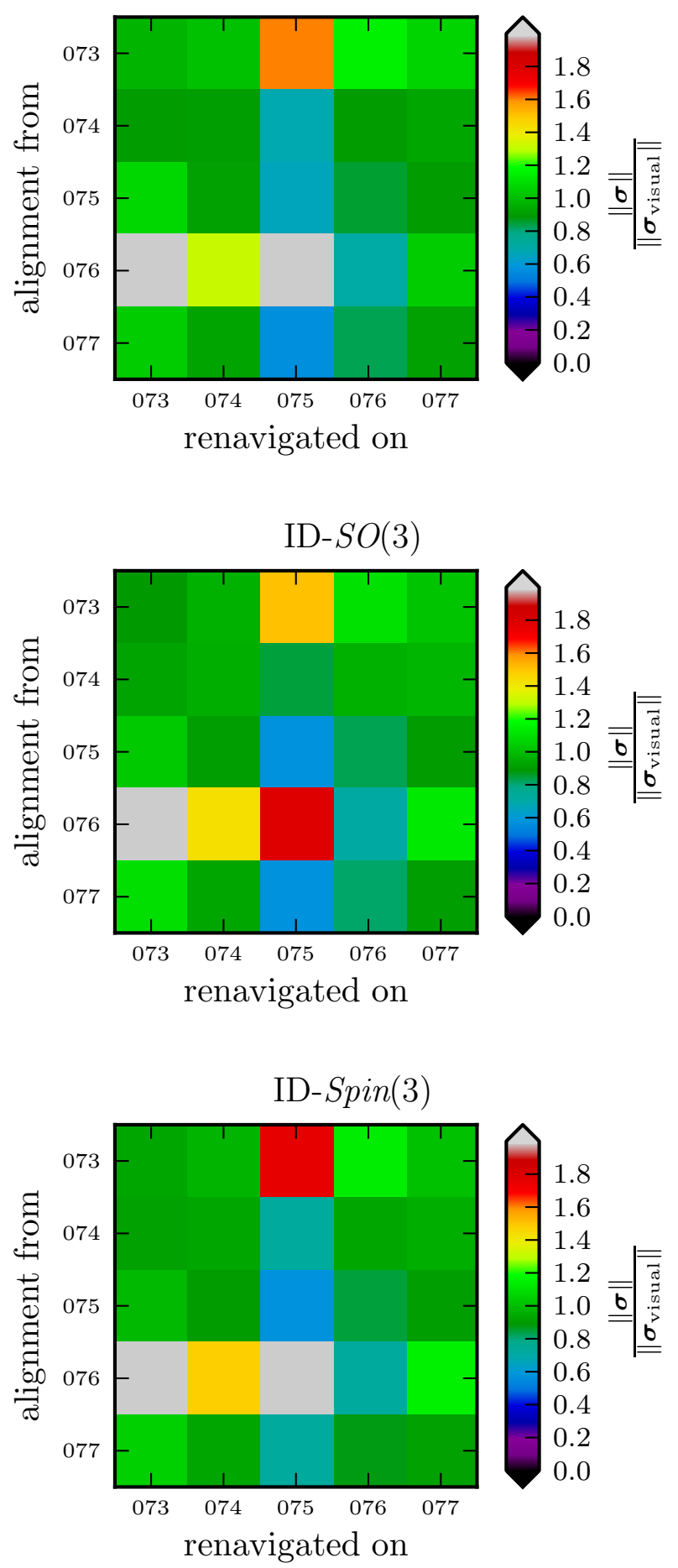

Figure 1-25: Cross-validation of alignment estimates on each other field dataset. Colors indicate the magnitude of the normalized standard deviation vector. 

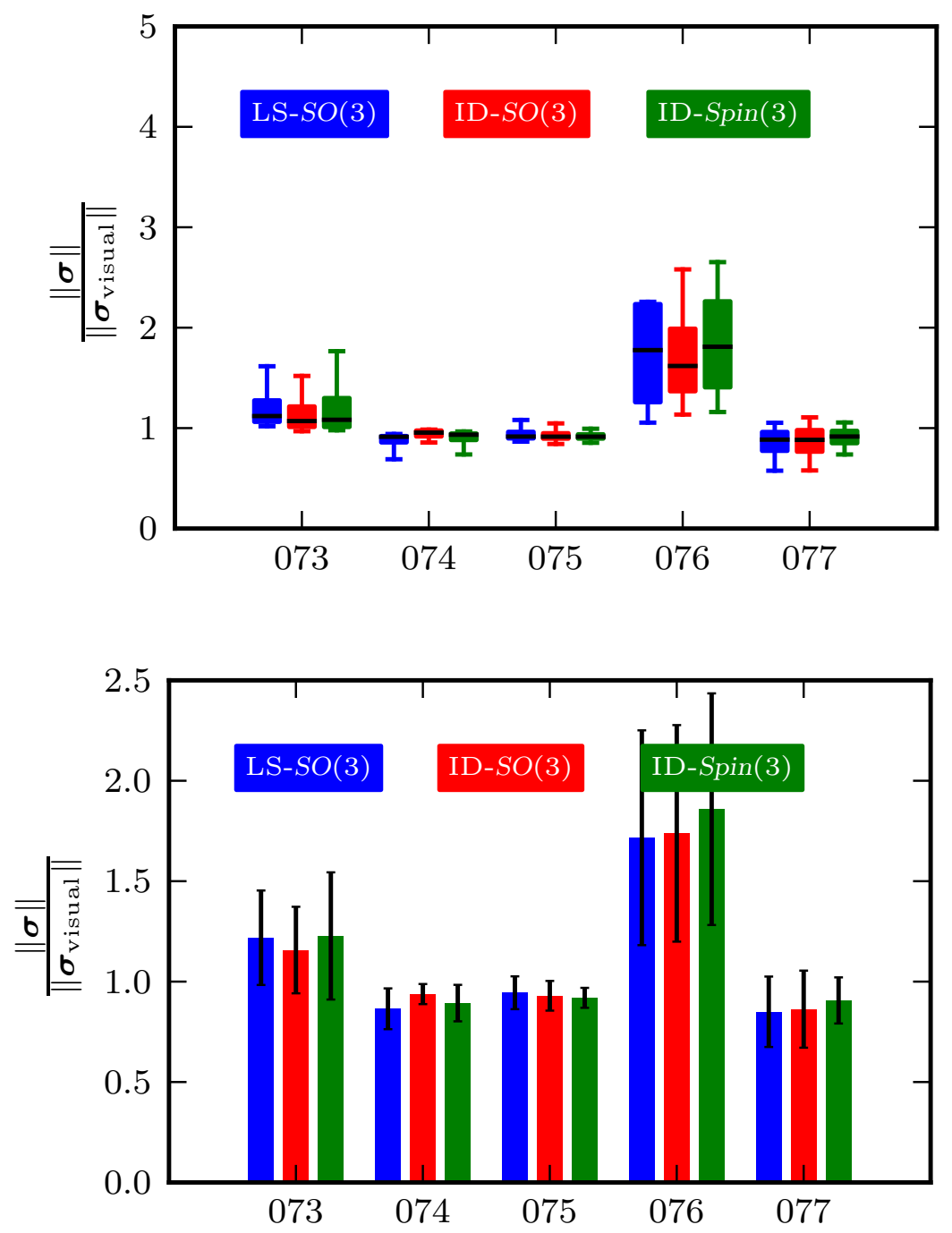

Figure 1-26: Cross-validation statistics of alignment estimates over all field datasets. 
structure implicitly constrains parameter estimates to describe rigid body rotations. We proposed a stable adaptive identifier in section 1.3, and proved its stability using Lyapunov theory on the continuous-time system. We then provided an equivalent discrete-time implementation of the identifier. Numerical simulations illustrated the behavior of the identifier under persistent excitation. These simulations showed that the identifier provides an accurate estimate of the true rotor, up to the level of noise in the input signals. Section 1.4.2 provided the necessary information to apply the rotor identifier to the DVL/FOG alignment problem for DR in underwater navigation, and defined several performance metrics. Section 1.5 applied the rotor identifier to identify DVL/FOG alignment on an ROV operating in controlled laboratory conditions, and section 1.6 demonstrated the rotor identifier using field data from the AUV Sentry. In the end, the rotor identifier improved DR performance comparably to previously reported rotation matrix methods. The Geometric Algebra formulation provides a clear kinematic interpretation of the proposed rotor identifier, which provides accurate, stable alignment estimates. It may offer a clearer path forward in improving online alignment identifiers, including, for example, enhanced noise rejection using a proportional-derivative feedback formulation. It may also prove useful in formulating an identifier for full rigid body motions-including rotation and translation-which can be encoded using rotors in higher dimensions. 


\section{Chapter 2}

\section{Dead Reckoning Through the Water Column}

\section{Hypothesis}

Relative water current profile data collected by a vehicle-mounted acoustic Doppler current profiler can provide estimates of the global vehicle velocity and position during descent and ascent phases of a mission.

\section{$2.1 \quad$ Introduction}

This chapter presents methods for dead reckoning through the water column, providing a navigation link between Global Positioning System (GPS) at the surface and dead reckoning (DR) at the seafloor, without relying on external systems. The methods use overlapping water profile measurements from an acoustic Doppler current profiler (ADCP) to simultaneously estimate the vehicle velocity and the ocean current profile.

Despite recent advances in technology and processing, underwater navigation remains a challenging problem. Signals from the GPS are only available at the beginning and the end of a mission, when the vehicle is on the surface. When it is near the seafloor, a vehicle can estimate its position by dead reckoning with a compass and a 
Doppler velocity log (DVL) in bottom-lock. In some cases, the vehicle can further improve its position estimate using information from the terrain (e.g., bathymetry or photography). Inertial navigation can provide good navigation in the short-term, but quality sensors are expensive and the solution is subject to drift in the long term.. There is a significant portion of each mission-during the descent and the ascent-where GPS and bottom-lock DVL measurements are unavailable, and the vehicle will drift in the ocean current.

Underwater vehicles currently rely on acoustic positioning methods for localization in the water column. Popular approaches include long baseline (LBL) transponder nets, or ship-mounted ultra-short baseline (USBL) tracking systems with an acoustic modem to downlink position fixes. However, these systems both require valuable ship time. This external dependence also limits the autonomy of the underwater vehicle. This chapter proposes a method to enhance the independent navigation capability of underwater vehicles, and discusses the challenges encountered in making this method work in the field.

The remainder of this section introduces the basic idea of DR using water profile data from an ADCP, then briefly reviews related work in the literature. Section 2.2 outlines the concept of operations. Section 2.3 gives details on the setup and implementation for three different approaches to solve the problem. Section 2.4 illustrates real-world performance and challenges using field data collected by the autonomous underwater vehicle (AUV) Sentry from Woods Hole Oceanographic Institution (WHOI). Finally, Section 2.5 summarizes the results of the field experiences and discusses the feasibility of an underwater navigation system operating entirely independent of external acoustic tracking. 


\subsubsection{To navigate from the surface to the seafloor and back}

Many underwater vehicles use a DVL for DR when they are near the seafloor ${ }^{1}$. The hardware and operating principles for a DVL and an ADCP are the same-comparing the Doppler shift of an acoustic signal along four different beams gives the velocity of an acoustic scatterer relative to the instrument. For a DVL in bottom-lock, the scatterer is the seafloor; for an ADCP, the scatterers are particles suspended in the water column. The same instrument can measure the relative velocity of the bottom and the ocean current.

Overlapping water current profiles measured by a vehicle-mounted ADCP can provide simultaneous estimates of the local ocean current and the vehicle velocity. This velocity can then be used for dead reckoning. It could also be used as an aiding input to an inertial navigation system (INS), but the method proposed here does not rely on an INS-it only requires an ADCP, depth sensor, and attitude reference. The goal is to develop a method that is applicable to many types of underwater vehicles with different grades of sensors, but flexible enough to be fused with other navigation methods where resources permit.

The approach presented here relies on three basic assumptions:

1. The vehicle starts or ends its transit with a global velocity estimate (e.g., from GPS or DVL bottom lock).

2. The vehicle can measure overlapping partial ocean current profiles relative to its own motion.

3. The ocean current may vary with depth, but the current at each depth is slowly varying in time. (i.e., The current is constant over the timescale of the ascent, on the order of minutes.)

If these conditions are satisfied, the vehicle can maintain an estimate of its global velocity as well as the ocean current as it transits through the water column.

\footnotetext{
${ }^{1}$ i.e., within the frequency dependent bottom-track range of the DVL: $\sim 246$ meters for a 300 $\mathrm{kHz}$ DVL, $\sim 94$ meters for a $600 \mathrm{kHz}$ DVL, or $\sim 25$ meters for a $1200 \mathrm{kHz}$ DVL (assuming $50 \mathrm{VDC}$ supply) [17].
} 


\subsubsection{Related Work}

The physical oceanography community has used ship-mounted ADCPs for many years to measure current profiles in the open ocean. In deep water the limited range ${ }^{2}$ of the ADCP prevents a ship-mounted instrument from obtaining a full current profile. Researchers address this shortcoming by mounting an ADCP on an instrument package that is lowered from the ship on a cable. The current profile from the Lowered Acoustic Doppler Current Profiler (LADCP) was initially computed by depth-averaging the vertical shear across the individual ensembles $[25,26]$. The present standard least squares (LS) batch processing method, proposed in [27], is more flexible for incorporating additional constraints, like bottom-track velocities and average velocity of the instrument package. This LADCP method is the standard approach to measure current profiles in the deep ocean.

Recently, there has been interest in using the DVL already onboard most deep submergence vehicles as an ADCP to simultaneously estimate vehicle velocity and ocean current. The vehicle velocity can then be used for DR through the water column. That is the goal of the research presented in this chapter. Preliminary work toward this goal was presented in in [28], demonstrating an online bin-average implementation in simulation and on field data collected by an AUV. An alternative approach, presented in [30], couples ADCP current profiles with inertial measurement unit (IMU) measurements in an information filter (IF) framework. This IF approach was later demonstrated on shallow field deployments with a high-frequency (1200 $\mathrm{kHz}$ ) ADCP in [31]. Field experiences navigating the ascent from several deep dives with a low-frequency $(300 \mathrm{kHz}) \mathrm{ADCP}$ are reported in [29], using the online bin average approach, as well as LS and recursive least squares (RLS) formulations of the problem.

\footnotetext{
${ }^{2}$ The nominal water profiling range of a $300 \mathrm{kHz}$ ADCP is 135 meters, but actual usable range depends on supply voltage and bin size [17].
} 
Table 2.1: Comparison of DR through the water column to related work. Filled circles denote features or characteristics posessed by the work, empty circles denote features that are missing or not demonstrated, and a dash means that category is not applicable.

\begin{tabular}{|c|c|c|c|c|c|c|c|c|}
\hline & \multicolumn{3}{|c|}{ measurements } & \multicolumn{3}{|c|}{ estimates } & \multirow[b]{2}{*}{ 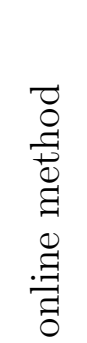 } & \multirow[b]{2}{*}{ 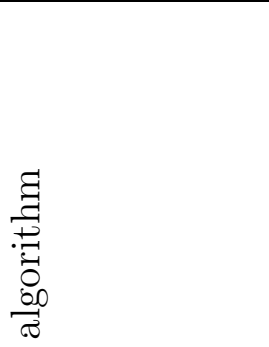 } \\
\hline & 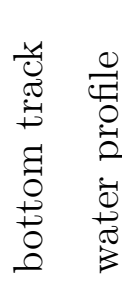 & 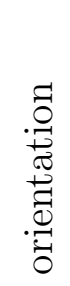 & 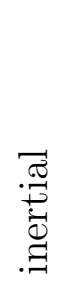 & 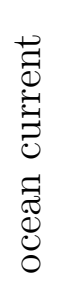 & 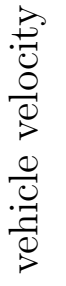 & 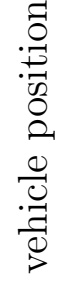 & & \\
\hline Firing [25] & $\circ$ & - & $\circ$ & - & $\circ$ & $\circ$ & $\circ$ & shear \\
\hline Fischer \& Visbeck [26] & o & - & o & - & $\circ$ & ○ & $\circ$ & shear \\
\hline Visbeck [27] & & $\bullet$ & $\circ$ & $\bullet$ & $\bullet$ & ○ & $\circ$ & LS \\
\hline Medagoda, et. al. $[30,31]$ & - $\bullet$ & $\bullet$ & $\bullet$ & $\bullet$ & $\bullet$ & $\bullet$ & $\bullet$ & IF \\
\hline Stanway $[28,29,87]$ & - $\bullet$ & - & o & - & - & - & - & avg., LS, RLS \\
\hline
\end{tabular}

\subsubsection{Contributions}

Table 2.1 compares this research to the related literature. Prior to beginning this research, there existed no methods for dead reckoning through the water column using current profiles measured by an ADCP. The initial contribution was simply recognizing the possibility that this could work. Designing and implementing the simple online bin average approach to the problem was the second contribution. This was originally reported in [28] and is detalied in section 2.3.1. The batch weighted least squares (WLS) approach to the LADCP problem was modified in [29] to handle systems with different size profile and ensemble bins-this is detailed in section 2.3.2. It was also adapted in [29] to an online approach using RLS, as detailed in section 2.3.3. Each of the methods is demonstrated on field data from ascents at the end of Sentry missions in section 2.4. The operational challenges reported in that section are also an important contribution to the topic of DR through the water column.

The work by Medagoda, et. al. [30,31] began independently, but at the same time as the research presented here. The main differences are that the problem is formulated within an IF, and tightly coupled to measurements from an INS ${ }^{3}$. The IF

\footnotetext{
${ }^{3}$ There is no fundamental reason that an IF could not be used without an INS, or that the online bin average, LS, and RLS methods presented here could not incorporate inertial measurements.
} 
formulation in $[30,31]$ and the bin-average and LS approaches presented here can be viewed as complementary approaches to the same problem.

\subsection{Concept of Operations}

Figure 2-1 illustrates the concept of DR through the water column during the descent phase of an underwater vehicle deployment. The vehicle starts at the surface with GPS and a partial observation of the current profile. This measurement, $\boldsymbol{u}_{w p}$, is a combination of the vehicle velocity $\boldsymbol{u}_{v}(t)$, the ocean current in each depth bin $\boldsymbol{u}_{c}(z)$, and measurement noise $\boldsymbol{u}_{n}(t)$ :

$$
\boldsymbol{u}_{w p}(t, z)=\boldsymbol{u}_{c}(z)-\boldsymbol{u}_{v}(t)+\boldsymbol{u}_{n}(t)
$$

As the vehicle descends, it loses GPS, but it observes a new current profile, which partially overlaps the earlier current profiles. The overlapping section contains information about the present vehicle velocity, in addition to refining the estimate of the ocean current at the overlapping depths. When the vehicle reaches the seafloor, it can use DVL bottom-track velocities to constrain the ocean current profile and then continue dead reckoning as usual.

\subsection{Implementation}

This section details three different approaches to solve the problem of DR through the water column: an online bin-average method, a batch WLS method, and a RLS method. Each approach has advantages and drawbacks. The batch WLS method is appropriate for postprocessing or when the vehicle only needs an estimate of its position upon reaching the seafloor or the surface. The online bin-average and RLS methods are more suitable for realtime navigation, especially on platforms with limited computational resources available for navigation (e.g., small AUVs or underwater gliders). 


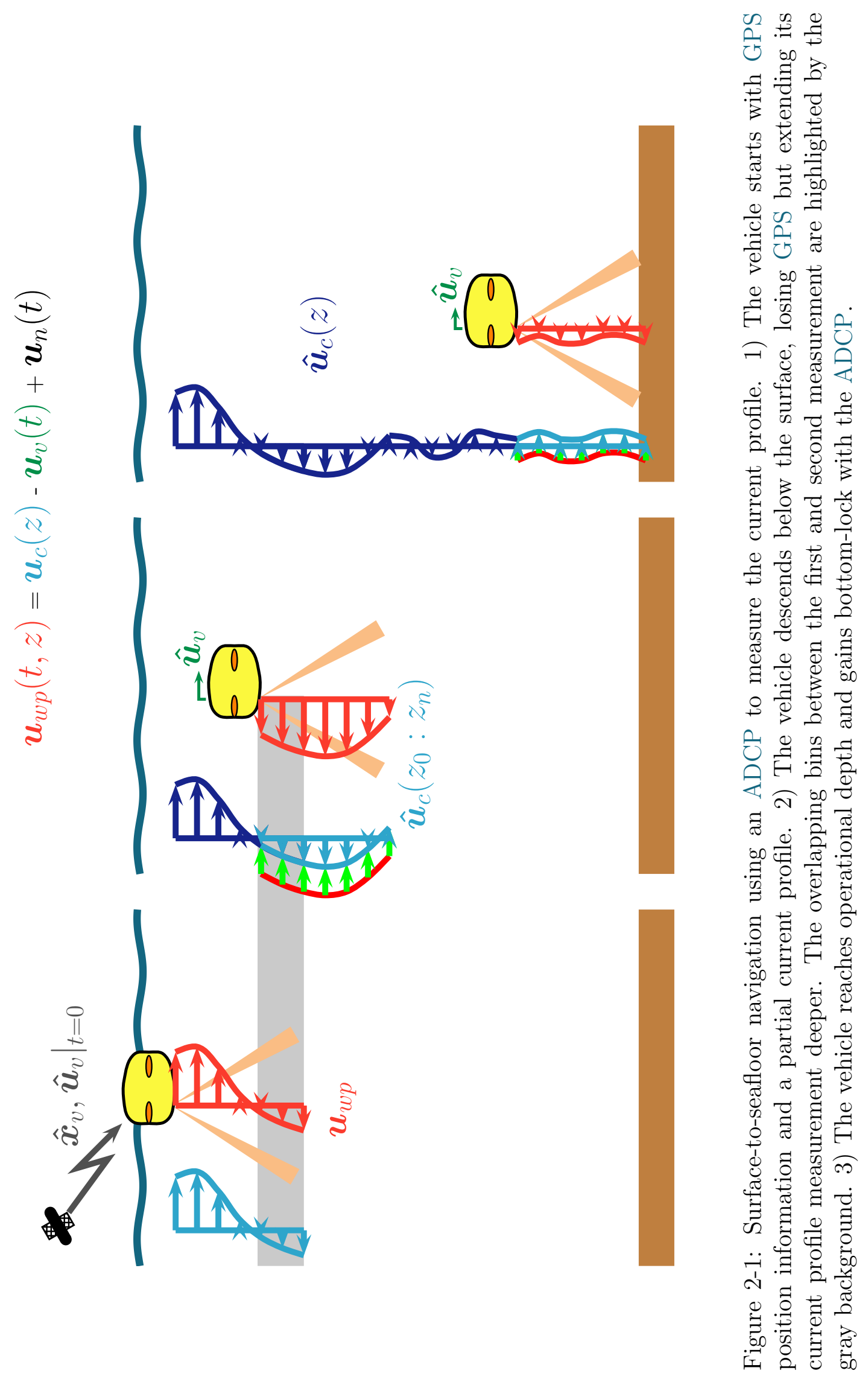




\subsubsection{Online bin-average}

This method is intended to be as computationally simple as possible while still enabling DR through the water column. Essentially, it estimates the vehicle velocity as the average difference between the observed velocity and the weighted average of past velocities, but only uses the observation bins that overlap with past observations.

\section{Algorithm}

Initialize the current estimate $\hat{\boldsymbol{u}}_{c}$ and bin weights $W$ at zero. The algorithm is then a simple loop for each ADCP measurement. Choose the correct depth $z_{i}$ for each current bin using the vehicle depth $z_{v}$ and the range to each measurement bin $r_{b_{i}}$ :

$$
z_{i}=z_{v}(t)+r_{b_{i}}, i=(0, \ldots, n)
$$

update the vehicle velocity estimate $\hat{\boldsymbol{u}}_{v}$ :

$$
\hat{\boldsymbol{u}}_{v}(t)=\frac{\sum_{i=0}^{n} W\left(z_{i}\right)\left(\hat{\boldsymbol{u}}_{c}\left(z_{i}\right)-\boldsymbol{u}_{m}\left(t, z_{i}\right)\right)}{\sum_{i=0}^{n} W\left(z_{i}\right)}
$$

update the water velocity estimate $\hat{\boldsymbol{u}}_{c}$ for each bin $i$ :

$$
\hat{\boldsymbol{u}}_{c}\left(z_{i}\right)=\frac{W\left(z_{i}\right) \hat{\boldsymbol{u}}_{c}\left(z_{i}\right)+\boldsymbol{u}_{m}\left(t, z_{i}\right)+\hat{\boldsymbol{u}}_{v}(t)}{W\left(z_{i}\right)+1}
$$

increment the bin weights $W$ :

$$
W\left(z_{i}\right)=W\left(z_{i}\right)+1
$$

integrate vehicle velocity to get vehicle position $\hat{\boldsymbol{x}}_{v}$ :

$$
\hat{\boldsymbol{x}}_{v}(t)=\hat{\boldsymbol{x}}_{v}(t-\delta t)+\int_{t-\delta t}^{t} \hat{\boldsymbol{u}}_{v}(\tau) \delta \tau
$$



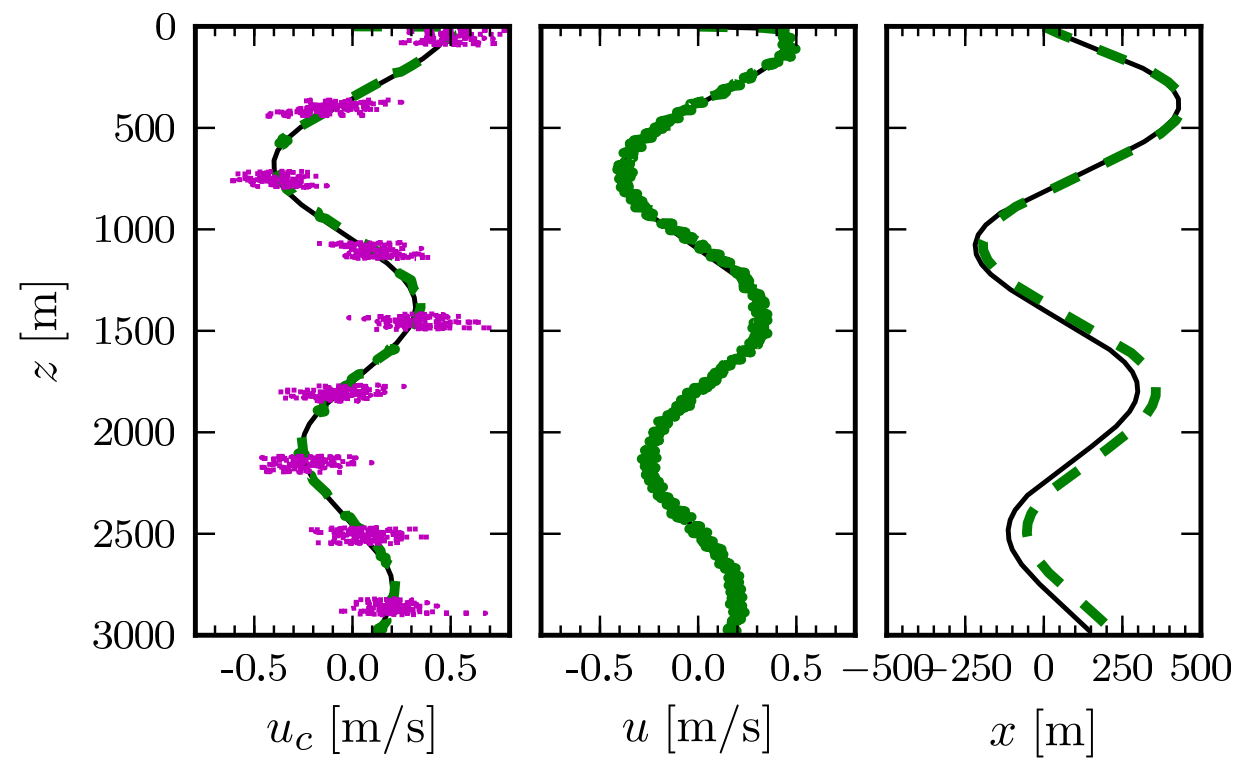

Figure 2-2: Current profile and vehicle state estimated by bin-average algorithm in simulation. The solid black line is the truth, and the dashed green line is the estimate. The magenta dotted segments on the current profile represent noisy single water profile measurements.

and repeat for each timestep and measurement ensemble. Note that this implementation uses the vehicle and water velocities in a stationary reference frame (e.g., North, East, Down), so the measured velocities must be rotated into this stationary frame using data from the attitude reference.

\section{Simulation}

The bin-average approach presented in the previous section was implemented in simulation using a simple vehicle descent model. The current was restricted to the $x$-direction, and modeled as a randomly generated decreasing sinusoid. The vehicle descended at a rate of $0.27 \mathrm{~m} / \mathrm{s}$, and was forced in the $x$-direction by the current. The simulation generated ADCP water profile measurements with 1-meter bin size and zero-mean, $\sigma=0.13 \mathrm{~m} / \mathrm{s}$, random noise.

Figure 2-2a shows that the current profile is identified well, while Figure 2-2b shows the estimated vehicle velocity follows the actual velocity closely. The actual and estimated vehicle position are shown in Figure 2-2c. The errors residuals for the estimated current profile, vehicle velocity, and dead-reckoned position are shown in 


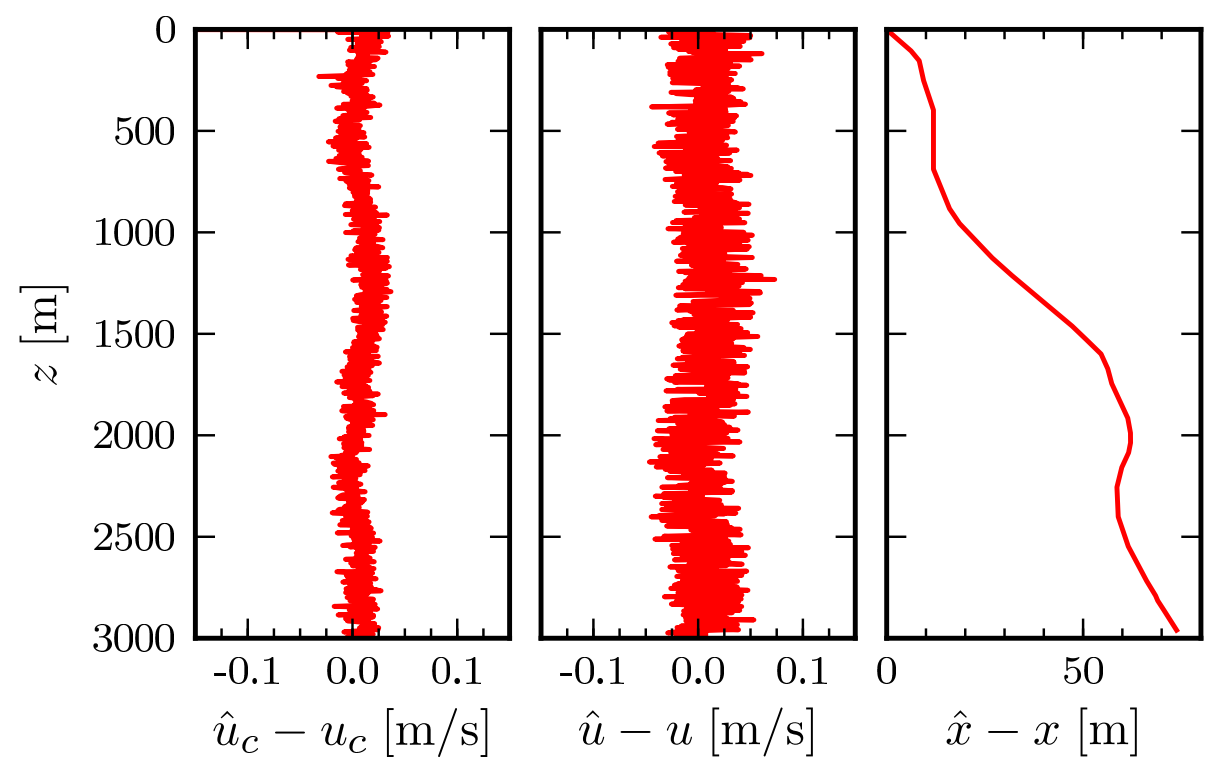

Figure 2-3: Error residuals in online bin average simulation results. The estimates of the current profile and vehicle velocity are reasonable, but noisy. The level of noise in both estimates is lower than the measurement noise in the single-ping ADCP water profiles. Since the position estimate is a result of DR, it is subject to integration drift. In this simulation, the final position error is $2-3 \%$ the length of the descent.

Table 2.2: Online bin-average water profile navigation error metrics.

\begin{tabular}{l|ccl}
\hline error in & $\mu$ & $\sigma$ & \\
\hline \hline$u_{v}$ & 0.00675 & 0.0163 & $\mathrm{~m} / \mathrm{s}$ \\
$u_{c}$ & 0.00574 & 0.0136 & $\mathrm{~m} / \mathrm{s}$ \\
$x_{v}$ & 39.8 & 23.1 & $\mathrm{~m}$ \\
\hline
\end{tabular}

Figure 2-3, and error statistics are given in Table 2.2.

It should be emphasized that this simulation restricted the current profile and vehicle velocity to the $x$-direction. It did not consider any effects of noise in the attitude sensor. The primary purpose of this simulation is to show that the ADCP water profile measurements and the simple online bin-average algorithm can provide adequate velocity estimates for dead reckoning. 


\subsubsection{Batch weighted least squares}

If the vehicle only needs to localize itself at the end of a water column transit ${ }^{4}$, the problem of DR though the water column is essentially equivalent to the LADCP problem, but focused on the vehicle velocities rather than the current profile. It can be formulated and solved as a LS or WLS problem [27]:

$$
\begin{aligned}
\mathbf{A x} & =\mathbf{b} \\
\mathbf{A}\left[\begin{array}{c}
\boldsymbol{u}_{c}\left(z_{0}\right) \\
\boldsymbol{u}_{c}\left(z_{1}\right) \\
\vdots \\
\boldsymbol{u}_{c}\left(z_{M}\right) \\
\boldsymbol{u}_{v}\left(t_{0}\right) \\
\boldsymbol{u}_{v}\left(t_{1}\right) \\
\vdots \\
\boldsymbol{u}_{v}\left(t_{N}\right)
\end{array}\right] & =\left[\begin{array}{c}
\boldsymbol{u}_{w p}\left(t_{0}, z_{0}\right) \\
\boldsymbol{u}_{w p}\left(t_{0}, z_{1}\right) \\
\vdots \\
\boldsymbol{u}_{w p}\left(t_{N}, z_{M}\right) \\
\boldsymbol{u}_{b t}\left(t_{0}\right) \\
\boldsymbol{u}_{b t}\left(t_{1}\right) \\
\vdots \\
\boldsymbol{u}_{b t}\left(t_{N}\right)
\end{array}\right],
\end{aligned}
$$

where $\mathbf{b}$ is the concatenation of the water profile measurement ensembles $\boldsymbol{u}_{w p}$ and bottom track measurement ensembles $\boldsymbol{u}_{b t}$. The influence matrix $\mathbf{A}$ couples the current profile and vehicle velocities in the solution $\mathbf{x}$ to the measurement data. The solution to this least squares problem minimizes the square of the estimation error:

$$
\hat{\mathbf{x}}=\underset{\mathbf{x}}{\operatorname{argmin}}\|\mathbf{b}-\mathbf{A x}\|^{2} .
$$

In a simple discretized problem with equal bin spacing for the ensembles and the profile, the influence matrix is sparse and filled by ones and zeros. More generally, the ensemble and profile bins will have different sizes. The implementation in this paper computes the influence of each current profile bin on each ensemble water profile bin based on the distance between the ensemble and profile bin center depths, divided by

\footnotetext{
${ }^{4}$ i.e., once it reaches the seafloor after a descent, or once it surfaces after an ascent.
} 
the profile bin spacing:

$$
a_{i, j}=\frac{1-\left|z_{e, i}-z_{p, j}\right|}{\Delta z_{p}}
$$

This works like a radial basis function with linear interpolation-one might also consider quadratic or Gaussian basis functions. All negative influence coefficients in the water profile block of the matrix are set to zero, and the sum of each row should be equal to one.

The upper right block of the influence matrix couples the ensemble water profile bins to the vehicle motion. Recalling the water profile measurement equation 2.1, the influence coefficients in this block are negative one wherever the vehicle velocity maps to an ensemble, and zero otherwise.

For ensembles with bottom track data, the influence matrix is expanded with a lower block. This is filled with zeros on the left, and a diagonal of negative ones on the right. The influence coefficients are negative here because the ADCP records bottom track velocities relative to a stationary instrument.

Figure 2-4 depicts an influence matrix for a simple example with thirteen profile bins spaced four meters apart. The example shows two measurement ensembles with four bins spaced at three meters and including bottom track data. The instrument is at 25 meters when the first ensemble is measured, and 23 meters when it measures the second.

Once the influence matrix has been set up, the problem can be solved using normal LS techniques. When one type of measurement is trusted more than another, WLS should be used. Each measurement is then weighted by the reciprocal of its standard deviation. (e.g., bottom-track velocities with $\sigma \sim 0.002 \mathrm{~m} / \mathrm{s}$ vs. water profile velocities with $\sigma \sim 0.02 \mathrm{~m} / \mathrm{s}$.)

\subsubsection{Recursive least squares}

The batch LS method in the previous section is suitable when the vehicle only needs to know its velocity and position at the end of a water column transit. When the vehicle needs incremental updates (e.g., for control) a recursive least squares (RLS) 


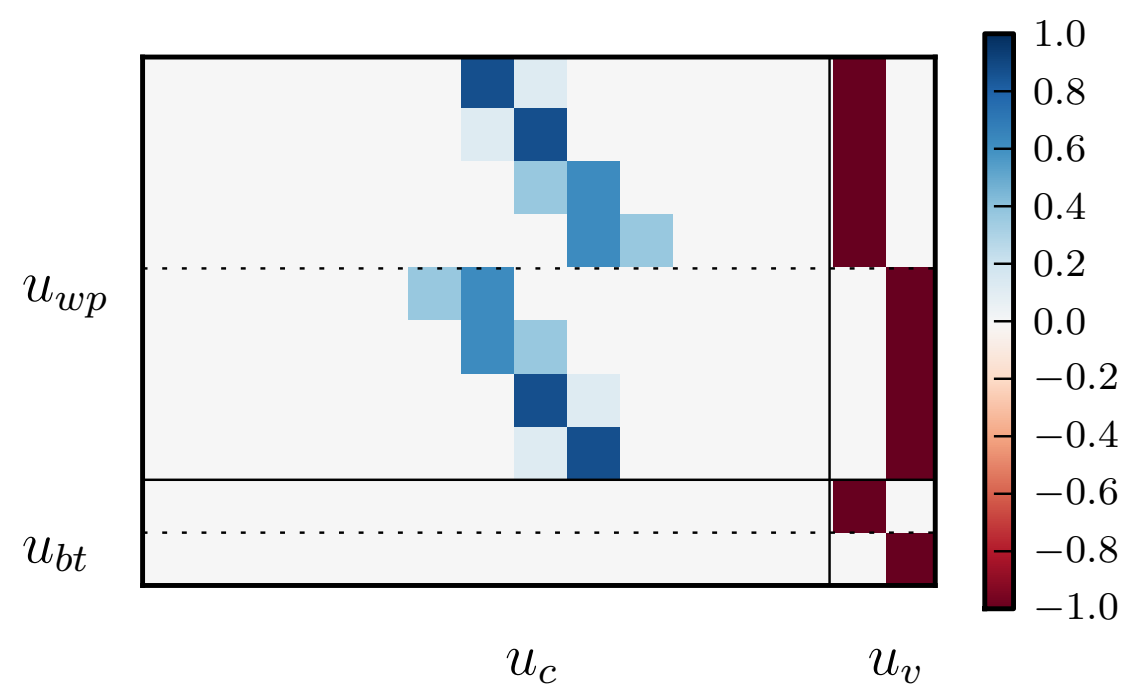

Figure 2-4: Influence matrix A for a simple system with 13 profile bins spaced 4 meters apart, and two measurement ensembles with 4 water profile bins spaced 3 meters apart, including bottom track data.

approach is more appropriate. This is also true when the memory available for the computation is limited, as in a low-power, long range AUV or glider.

Recursive least squares depends on the ability to track an estimate of the state $\mathbf{x}$ and its error covariance $\mathbf{P}$. With this information, an optimal gain can be calculated any time new data is available, without starting the whole calculation from scratch [56].

The innovation $\boldsymbol{\nu}_{k}$ in the new data is defined as:

$$
\boldsymbol{\nu}_{k}=\mathbf{b}_{k}-\mathbf{A}_{k} \mathbf{x}_{k-1}
$$

Given the measurement covariance matrix $\mathbf{R}_{k}$, the optimal gain can be calculated $a$ priori:

$$
\mathbf{K}_{k}=\mathbf{P}_{k-1} \mathbf{A}_{k}^{T}\left(\mathbf{A}_{k} \mathbf{P}_{k-1} \mathbf{A}_{k}^{T}+\mathbf{R}_{k}\right)^{-1}
$$


so that the updated state estimate is:

$$
\mathbf{x}_{k+1}=\mathbf{x}_{k}+\mathbf{K}_{k} \boldsymbol{\nu}_{k}
$$

and the updated covariance is:

$$
\mathbf{P}_{k}=\left(\mathbf{I}-\mathbf{K}_{k} \mathbf{A}_{k}\right) \mathbf{P}_{k-1}
$$

This form of RLS estimator essentially repeats the measurement update step of the Kalman filter (KF), without the process update [88]. In signal processing literature, equation 2.5 is sometimes called the 'data matrix form' of the LS estimation problem [89], so equations 2.7-2.10 can be considered the 'data matrix form' of the RLS filter or estimator.

In this research, the RLS estimator is implemented by initializing a full solution vector $\mathbf{x}_{0}$ of zeros, and calculating the influence matrix $\mathbf{A}_{k}$ for each measurement ensemble based on the measured depth of the instrument. An alternate implementation might only store the instrument velocities at the current timestep, and include a process update equation-this would produce an augmented state Kalman filter (KF).

\section{$2.4 \quad$ Field Experiences}

This section discusses some of the operational and practical challenges involved in measuring water current profiles from the AUV Sentry. Several field deployments illustrate lessons learned with respect to ADCP configuration and data quality. We also present estimated current profiles and dead reckoned vehicle trajectories for each dive. We compare these estimates to position fixes measured by external acoustic tracking systems. 


\subsubsection{The AUV Sentry}

Sentry is a deep submergence AUV operated by the WHOI DSL. It is the successor to the autonomous benthic explorer (ABE) [48], with many design improvements to address the needs of the oceanographic community. Sentry mission tasks include highresolution bathymetry, seafloor photography, geomagnetics, and chemical sensing.

Sentry's navigation sensor suite includes: an INS with fiber-optic gyroscope (IXSEA PHINS), a $300 \mathrm{kHz}$ DVL/ADCP (Teledyne RD Instruments Workhorse Navigator), a pressure sensor (Paroscientific Digiquartz), an LBL positioning system (WHOI), a USBL tracking system (Sonardyne), and a backup magnetic compass (PNI). The standard science sensor suite includes: a conductivity/temperature probe, precision magnetometers, a $400 \mathrm{kHz}$ multibeam sonar, and a 12-bit camera. Sentry has also carried redox potential (eH) probes, dissolved oxygen sensors, an in-situ mass spectrometer, a sub-bottom profiler, and sidescan sonar.

\section{Working with multiple sonars}

High-resolution bathymetric mapping is one of the primary science tasks for Sentry. The $400 \mathrm{kHz}$ multibeam sonar collects soundings of the seafloor, which are combined with vehicle navigation to produce detailed maps for scientists and for joint operations with other vehicles (e.g., DSV Alvin or ROV Jason).

This presents a minor challenge for DR with the $300 \mathrm{kHz}$ Doppler sonar-the broadband signal can show up in the multibeam soundings. Careful timing and a low-latency trigger input allow interleaving so that the multibeam and Doppler signals do not interfere with each other. However, this also constrains the repetition rate of both sensors. The nominal sample rate of the sonars is $2 \mathrm{~Hz}$ during standard operations.

\section{Navigated ascent}

When configured to measure both bottom track and water profile data, the ADCP actually uses two different types of pings for each measurement ensemble. The bottom 
track ping is longer and the seafloor is a reasonably strong acoustic scatterer, so the ADCP can produce high accuracy velocity measurements at relatively long range. The length of the water profile ping is limited by the ensemble bin spacing. These two types of pings are interleaved when the ADCP collects both bottom track and water profile data. Even if the ensemble has just one ping of each type, this halves the effective data rate. The bottom-track ping is sent first, and the ensemble data is reported after the water profile return is received, introducing additional latency.

This lower data rate and measurement latency could potentially degrade the performance of Sentry's navigation during the main mission, so the data sets for DR through the water column are currently restricted to the ascent.

\section{Doppler sonar configuration}

The ADCP has many parameters that can be adjusted in its firmware. However, these settings cannot be altered online-the instrument must stop recording data any time a parameter needs to be changed.

The water profile settings are important for navigating through the water column. The ADCP is set to record only one ping per ensemble so that it reports data at its highest rate. The correlation magnitude threshold and echo intensity threshold are both set to zero so that the ADCP does not throw out any data on its own. This means that the data will need to be prefiltered before using it for navigation, but it also ensures that we have the data needed for developing and tuning the navigation

process. The number and spacing of the water profile bins were varied between dives, and the actual values are noted in the following subsections.

\subsubsection{Sentry 059: Galápagos Ridge}

Sentry dive 059 was a bathymetry and photography mission on the Galápagos ridge during the GRUVEE2010 research cruise led by chief scientist John Sinton. At the end of the mission, Sentry ascended from 1600 meters with an average depth rate of $-0.62 \mathrm{~m} / \mathrm{s}$. The ADCP interleaved bottom pings and water pings for the entire 


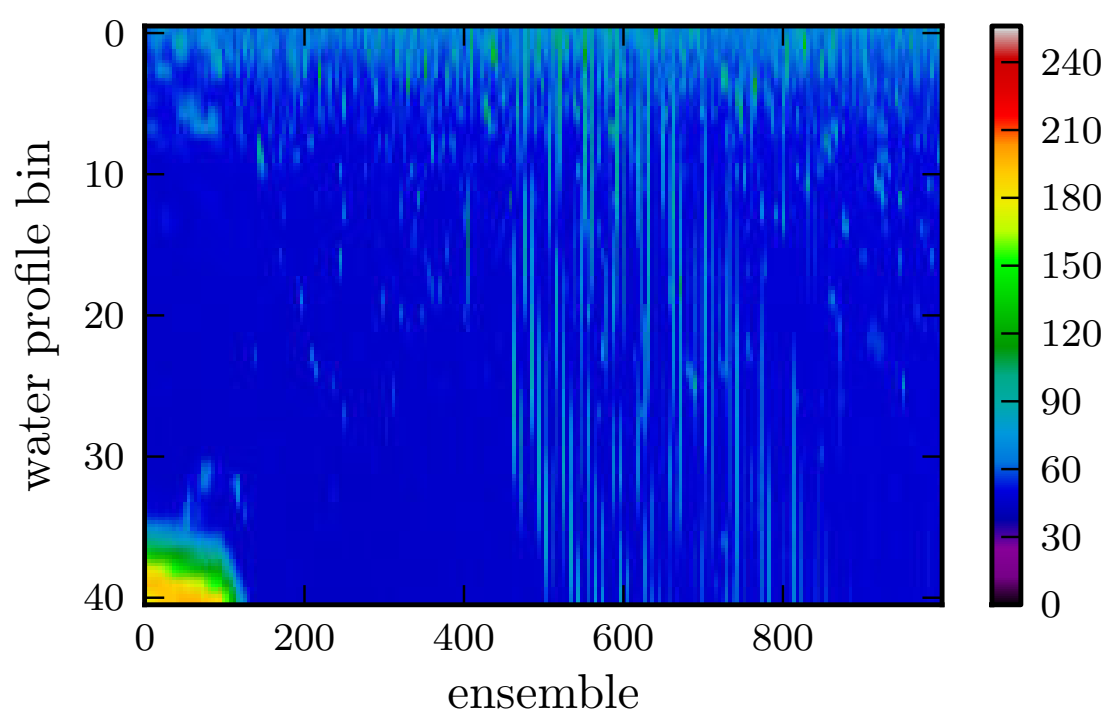

Figure 2-5: Echo intensity from water profile measurements during ascent from Sentry dive 059. Note the bands of slightly increased echo intensity in ensembles 450-850.

ascent. Each ensemble measured 41 water profile bins at a spacing of 2 meters. Low echo intensity and correlation magnitude, coupled with high error velocity, meant that much of the data beyond bin 20 was ignored.

\section{Bottom-track interference}

Figures 2-5, 2-6, and 2-7 show the echo intensity, correlation magnitude, and error velocity of the water profile pings from beam 1 at the beginning of the ascent. Significant interference is visible in ensembles 450 through 850, marked by increased echo intensity and error velocity, with irregular correlation magnitude.

The ADCP measured these ensembles 6.25-9.5 minutes into the ascent, starting just after Sentry ascended past 230 meters above the seafloor. This is also at the limit of the bottom-track range. When it loses bottom-lock, the ADCP begins searching for the bottom again, but the vehicle continues to ascend and the instrument stays out of range.

Late returns from bottom track pings are the likely cause of interference in these ensembles. It is possible that the internal theshold filters of the ADCP, limiting the range of acceptable correlation magnitude or error velocity, would reduce this 


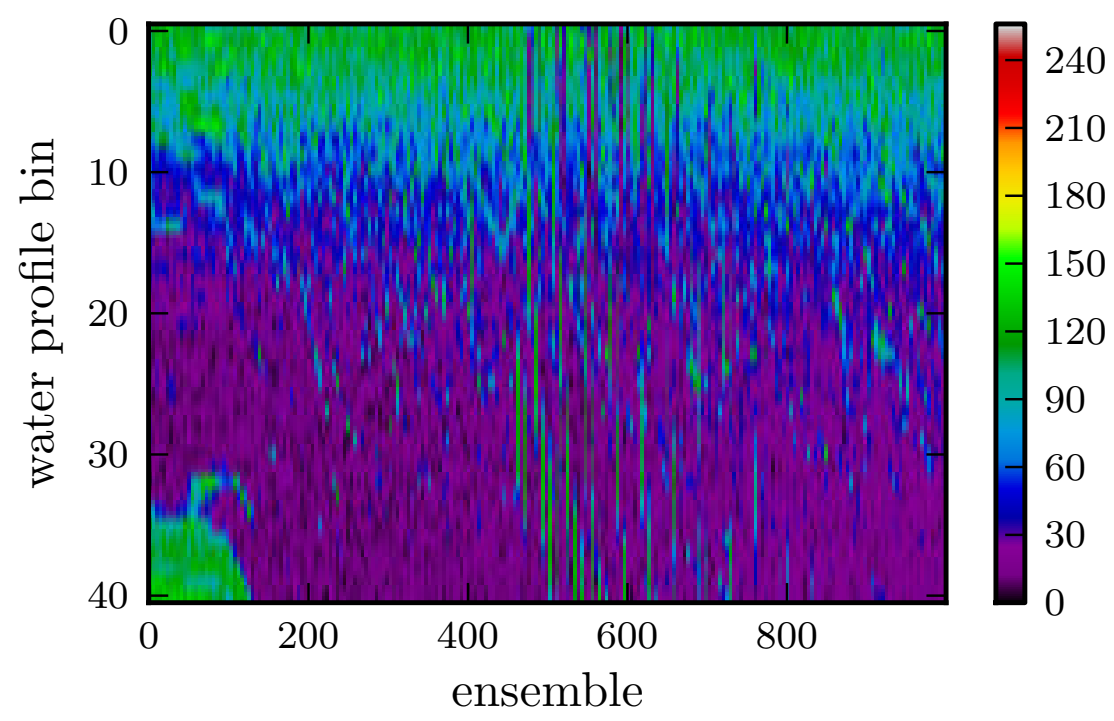

Figure 2-6: Correlation magnitude from water profile measurements during ascent from Sentry dive 059. Note the decreased correlation magnitude in near bins (010) during ensembles 450-650. The correlation magnitude is increased in outer bins (20-40) for the same timeframe and extending to ensemble 800.

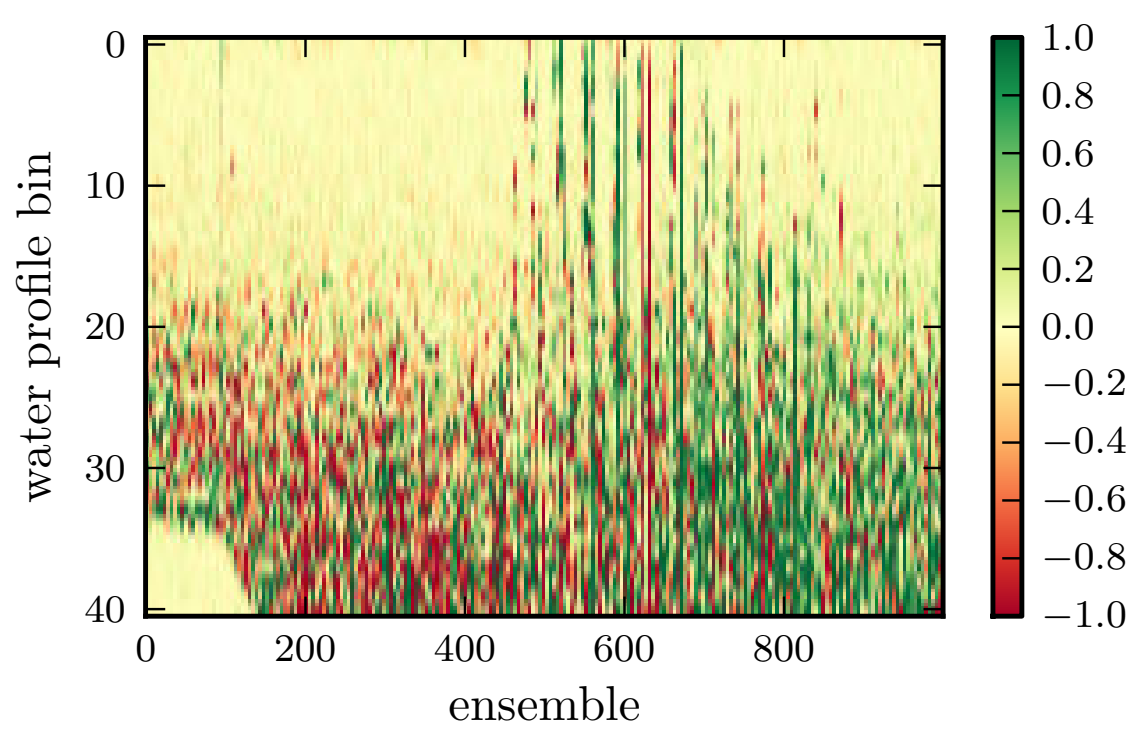

Figure 2-7: Error velocity from water profile measurements during ascent from Sentry dive 059. The magnitude of the error velocity is high for almost measurements further out than bin 20, and there are bands of incresed error velocity nearer than bin 20 for ensembles 450-850. 


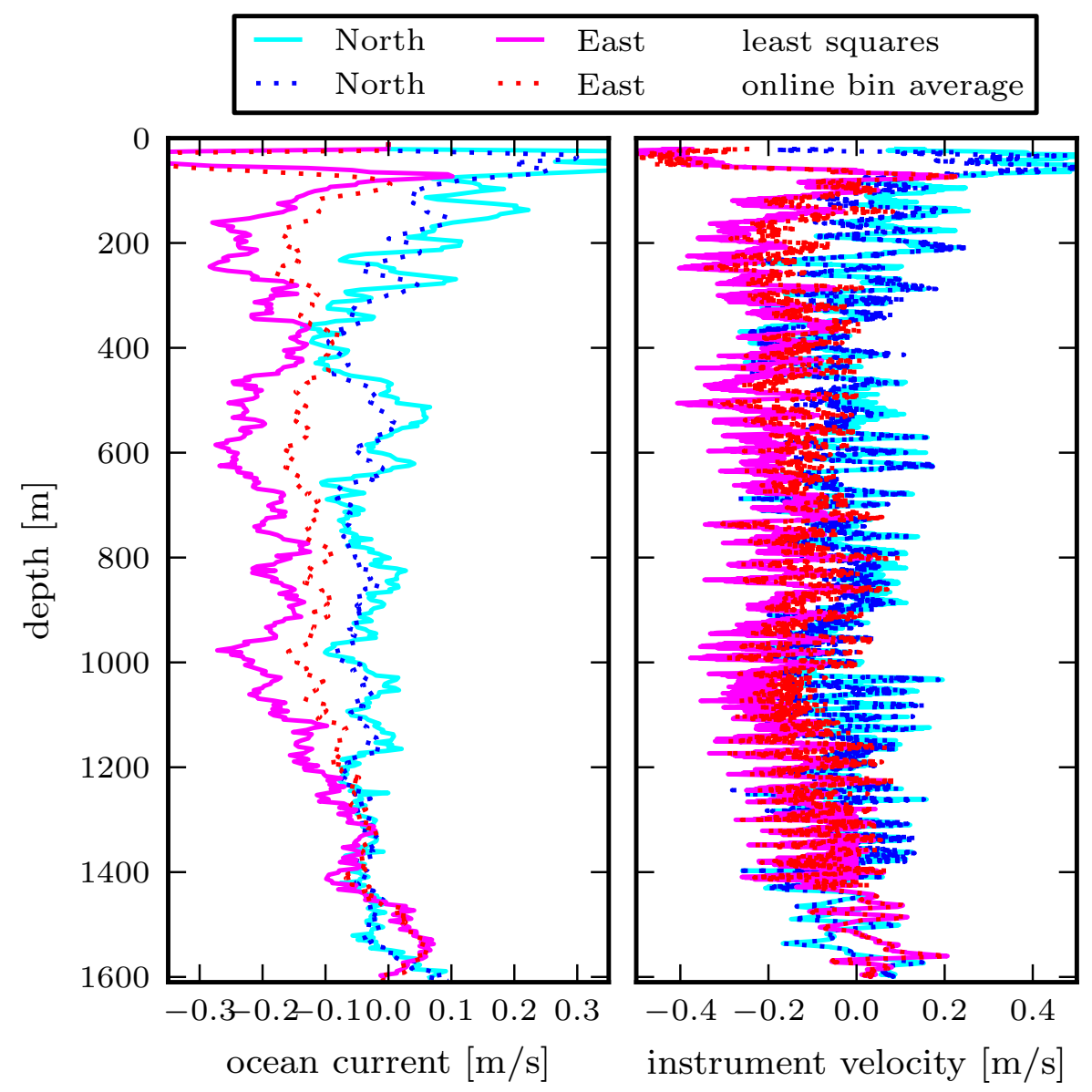

Figure 2-8: Estimated ocean current profile and vehicle velocity during ascent from Sentry dive 059 .

interference, but that does not work for this dataset.

Figure 2-8 shows the estimated current profile and vehicle velocity when the input data are limited to those with error velocity less than $0.1 \mathrm{~m} / \mathrm{s}$, and correlation magnitude greater than 72. The online bin average and LS estimates of the northward current and instrument velocity are similar, but the eastward components are very different in some places. Figure 2-9 plots the resulting vehicle trajectory estimates, with corresponding USBL position fixes for comparison. It seems likely that the eastward velocities estimated by LS are more correct, but it is also clear that this dataset does not provide good overall navigation. Later dives used different ADCP parameters to improve echo intensity and correlation magnitude. 


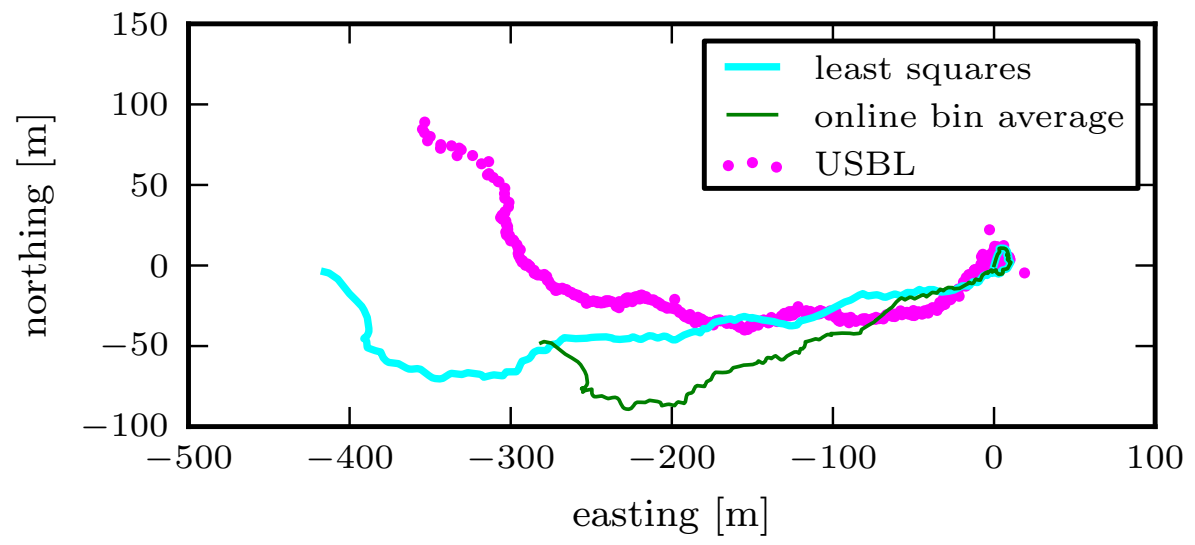

Figure 2-9: Estimated vehicle trajectory during ascent from Sentry dive 059.

\subsubsection{Sentry069: Juan de Fuca}

Sentry dive 069 was a 500 meter deep bathymetry mission on Hydrate Ridge during the ENLIGHTEN10 research cruise led by chief scientist John Delaney. Sentry interleaved bottom pings and water pings for the first 5 minutes of the ascent. There was a brief data gap while the ADCP was reconfigured to stop collecting bottom track data. This was an attempt to avoid the possible bottom-track interference encountered in earlier ascents. Each ensemble measured 21 water profile bins at a spacing of 3 meters.

Figure 2-10 plots the estimated ocean current profile and instrument velocity through the ascent. The estimates from batch LS, RLS, and the online bin average approach are in close agreement. The ADCP data quality for this dive was much higher, primarily due to the larger ensemble bin spacing, which led to increased echo intensity and correlation magnitude with decreased error velocities. This comes at the price of lower spatial resolution, but almost none of the data had to be thrown out as it had in dive 059 .

The integrated vehicle trajectory is shown in figure 2-11. Again, the estimates provided by the three different approaches are nearly identical. Position fixes from 


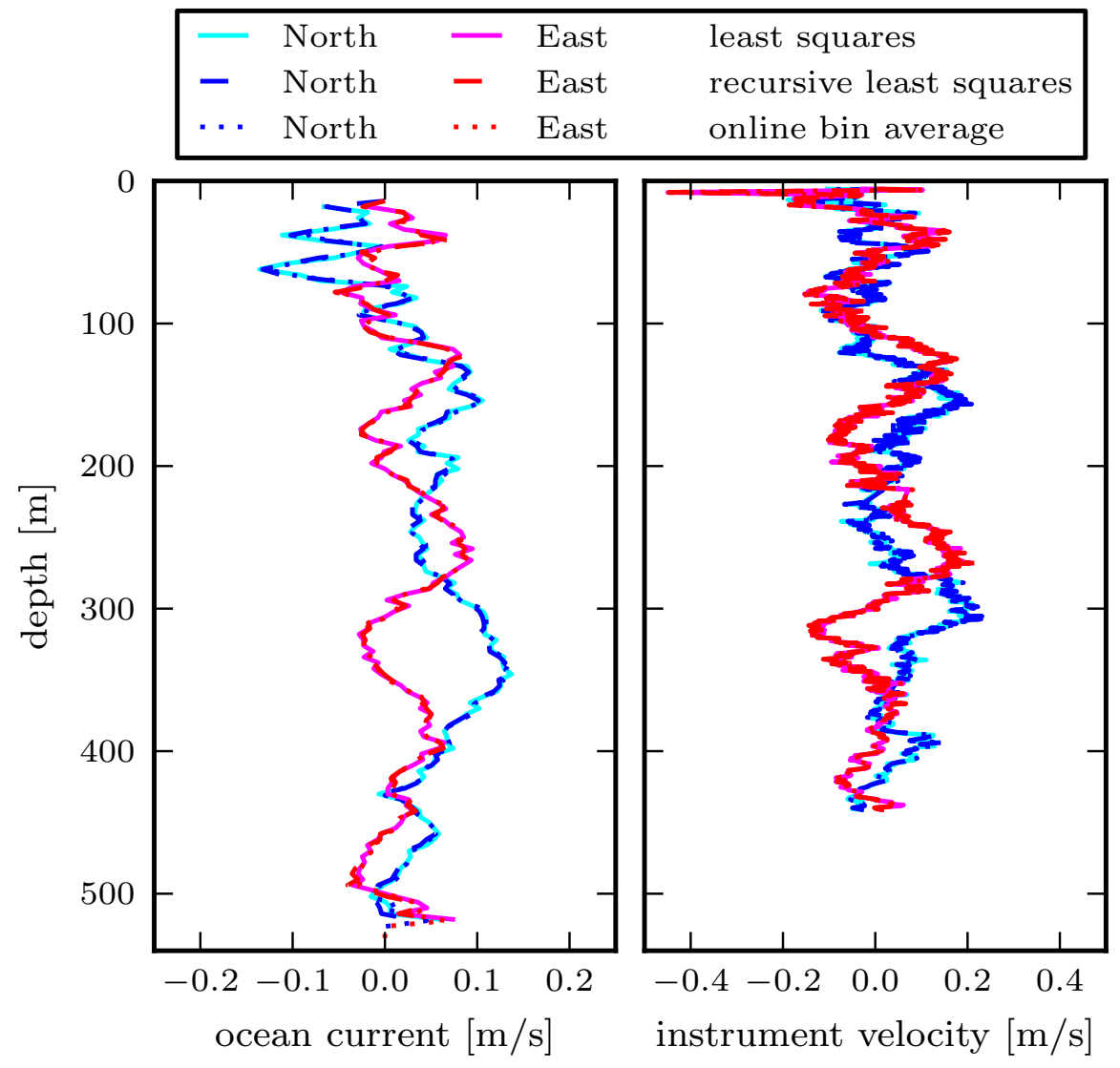

Figure 2-10: Estimated ocean current profile and instrument velocity during ascent from Sentry dive 069. The estimates from batch LS, RLS, and the online bin average approach are indistinguishable at this scale on this relatively shallow dive. 


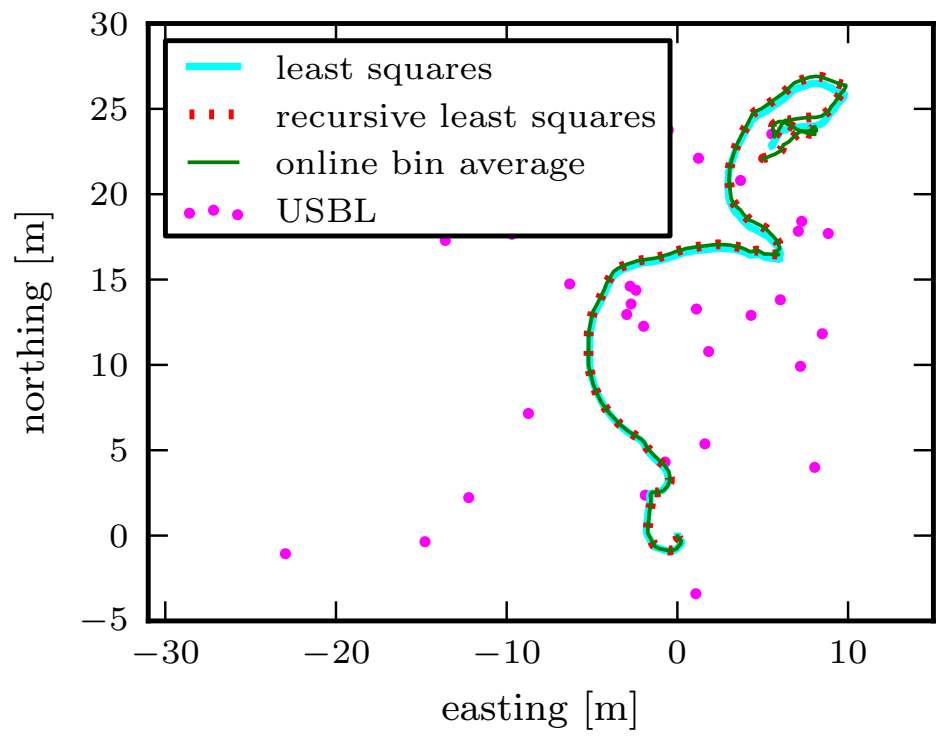

Figure 2-11: Estimated vehicle trajectory during ascent from Sentry dive 069. The three dead reckoning methods agree closely. Unfortunately, the USBL data are widely scattered and do not provide a good basis for comparison during this short ascent.

the shipboard USBL system are also shown, but unfortunately these data are widely scattered and poor quality during the short ascent.

\subsubsection{Sentry085: Kermadec Arch}

Dive 085 was a bathymetry mission to 1000 meters depth off the coast of of New Zealand during a research cruise led by chief scientist Cornel de Ronde.

Again, Sentry interleaved bottom pings and water pings for the first 5 minutes of the ascent. Each ensemble measured 27 water profile bins at a spacing of 3 meters. There was a brief data gap while the ADCP was reconfigured to stop collecting bottom track data.

This dataset highlights one of the weaknesses of the simple online bin average approach-a few bits of bad data when the instrument was near 800 meters deep have thrown off the ocean current estimate (and thus the instrument velocity measurement) for the remainder of the ascent. The LS estimator was less suceptible.

The integrated position estimates tell a similar story-the LS trajectory is qualitatively similar to the shape traced out by the USBL fixes. Figure 2-13 shows a 


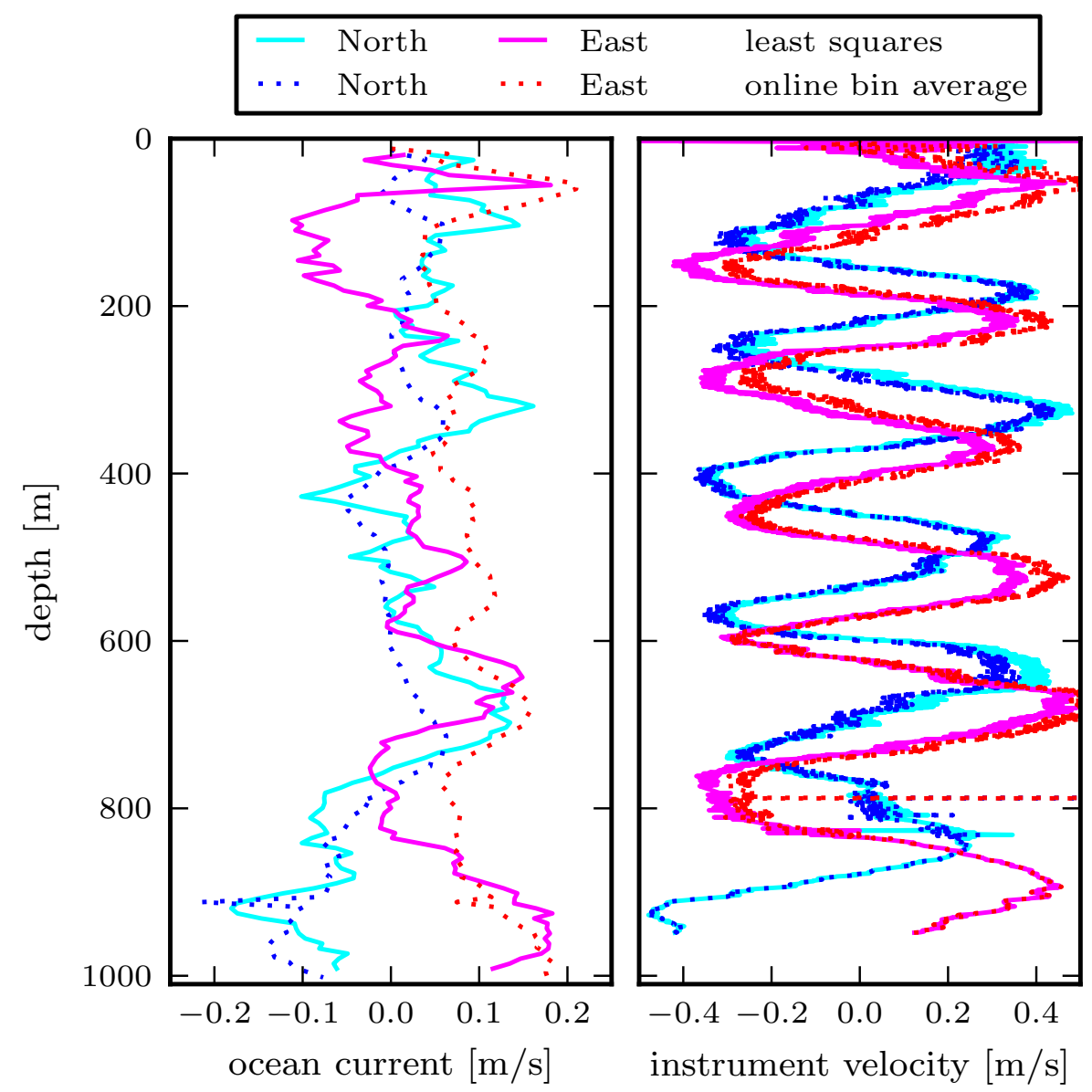

Figure 2-12: Estimated ocean current profile and vehicle velocity during ascent from Sentry dive 085. (RLS estimates are not plotted because they are nearly identical to the LS.) The online bin average estimated instrument velocity shows an anomaly near 800 meters depth. This highlights a weakness in that method-a few bits of bad data can cascade to throw off the entire estimated ascent. 


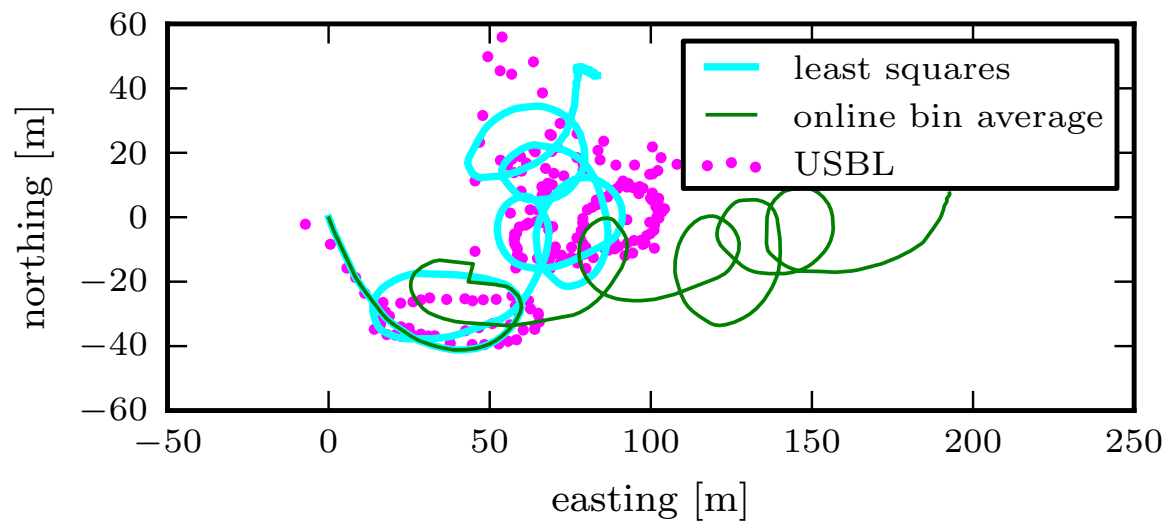

Figure 2-13: Estimated vehicle trajectory during ascent from Sentry dive 085.

map-view of the ascent trajectory, while Figure 2-14 plots the individual North and East components.

\subsection{Discussion and Conclusions}

This chapter has proposed a method for dead reckoning through the water column using overlapping water profile measurements collected by a vehicle-mounted ADCP. Field experiences with the deep submervence AUV Sentry, using a $300 \mathrm{kHz}$ ADCP, have shown that there are some practical challenges to address before this technique is ready for serious use in autonomous navigation. High data quality must be provided throughout the entire water column transit-this may be enabled in the future by adapting ADCP configuration parameters on-the-fly. It would be very advantageous to be able to avoid the interference from searching for bottom-track by disabling it without needing to stop water pings.

Although this technique is not quite ready for field service on deep AUVs, it holds great promise. Dead reckoning using ADCP water profile measurements enhances the autonomy of underwater vehicles because it enables underwater navigation without dependence on external systems. It provides a link between GPS at the surface and 

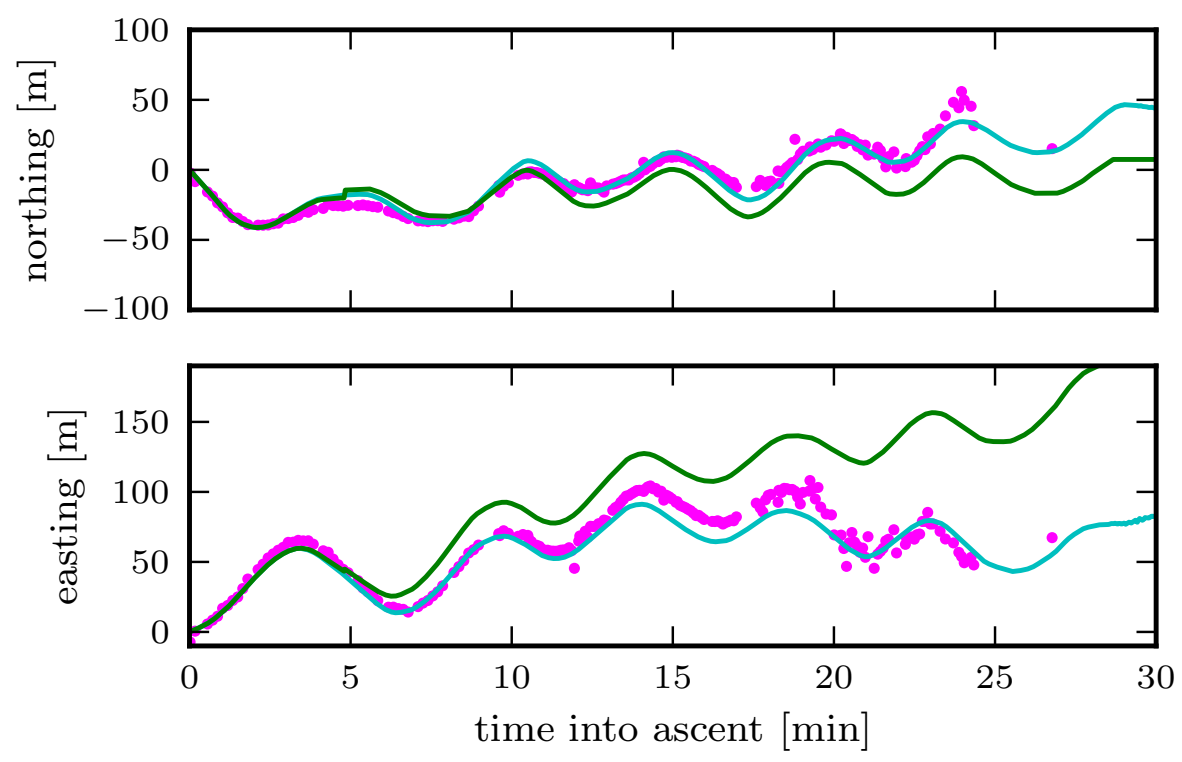

Figure 2-14: North and East components of estimated vehicle trajectory during ascent from Sentry dive 085 .

DR or terrain-relative navigation near the seafloor. 


\section{Chapter 3}

\section{Model-Driven Delayed Measurement Fusion}

\section{Hypothesis}

Mathematically rigorous treatment of measurement delays in multi-sensor fusion Kalman filters can be achieved through state augmentation and conditional timevarying models without altering the underlying framework.

\subsection{Introduction}

If a measurement is delayed in time, how should a realtime state estimator process use the information contained in that measurement to update the current state estimate? This chapter presents a flexible, model-driven approach to efficiently and effectively fuse delayed measurements in realtime. The specific motivation for this study is acoustic position aiding for underwater navigation.

Efficiency and effectiveness are two important properties for a realtime state estimator. In this context, efficiency means that the fusion process should avoid duplicated effort (i.e., repeated computations) whenever and wherever possible. We also want to avoid increasing the dimension of the problem (i.e., the length of the state vector) any more than necessary. We only want to carry enough extra information to 
fuse the delayed measurement in a principled manner, and leave behind any information we won't use. Effectiveness means (at least) that fusing a delayed measurement should reduce the uncertainty in the state estimate. It can also relate to the ease of operating and designing the filter. The model-driven approach presented here handles delayed measurements more efficiently and effectively than previous methods.

The remainder of this section introduces the general problem in more detail. Section 3.1.1 outlines the common methods for measuring position underwater, and goes into more detail on two acoustic tracking methods that will serve as application examples for the proposed delayed measurement fusion approach. Section 3.1.2 briefly reviews related work, and section 3.1.3 enumerates the contributions of this research.

We classify and briefly discuss different types of measurement delays in section 3.2. We present the details of model-driven delayed measurement fusion in section 3.3, reviewing some key concepts of the Kalman filter before proposing two specific modifications to compensate for measurement delay. Section 3.4 walks through a canonical example, using a damped harmonic oscillator with delayed position measurements to illustrate the process proposed here.

Then we apply the proposed model-driven delayed measurement fusion approach

to the real-world problem of underwater navigation. We present ultra-short baseline (USBL)-aided dead reckoning (DR) as a loosely coupled example in section 3.5, and long baseline (LBL)-aided DR as a more tightly integrated example in section 3.6. Both examples are demonstrated on field data from the National Deep Submergence Facility (NDSF) autonomous underwater vehicle (AUV) Sentry.

\subsubsection{Delayed measurements in underwater positioning}

For oceanographic underwater vehicles, it is as important to know the location where measurements or samples were collected as it is to collect the data in the first place. Localization is also important in most military and offshore industry applications. After all, knowing where you are is often the first step in getting where you need to be.

Precise, robust underwater navigation relies on many measurements, fused in an 
intelligent way, to produce an accurate state estimate. Realtime navigation relies on DR-measuring vehicle orientation with a compass or gyrocompass and measuring vehicle velocity with a Doppler velocity log (DVL) or estimating it from a dynamic model. This is essentially integrating the first-order kinematics of the vehicle forward in time. Inertial navigation integrates forward from the second-order kinematicsusing accelerometers to measure acceleration, and gyroscopes to measure angular rates. These are both attractive options because they require no external infrastructure and can operate at high update rates. However, without position measurements, any inertial navigation or dead reckoning system will accumulate error and uncertainty over time. Including position aiding makes the navigation solution more accurate and robust in the long term.

Since the radio frequency signals of Global Positioning System (GPS) do not penetrate the ocean surface, underwater navigators must rely on other means to determine position. Depth is determined from measurements of pressure, temperature, and conductivity [2]. Long baseline (LBL), short baseline (SBL), or ultra-short baseline (USBL) acoustic positioning systems provide measurements in the NorthEast plane. More recently, terrain-relative techniques using bathymetry and/or photography have been developed to provide relative position measurements [38, 45, 90] for navigation aiding.

The methods summarized above all provide relative position information in the local navigation frame. They must be geo-referenced to provide positions in the global frame: LBL beacons must be surveyed, SBL and USBL systems must be coupled to GPS and attitude sensors, and terrain-relative navigation must be over known terrain or have known boundary conditions.

With the exception of depth measurements, all of these methods for determining position have inherent latency. There is a finite delay between the time a measurement is valid and the time it is available. The delays associated with $(x, y)$ position measurements underwater are on the order of seconds, and in some cases longer. It is important to understand these delays, and properly account for them, in order to achieve precise and robust navigation. 


\subsubsection{Related Work}

\section{The Kalman filter}

The Kalman filter (KF) [91] is an optimal recursive estimator for a linear system with stochastic inputs. It fuses state measurements of a system with estimates provided by a model of that system in proportion to the covariances of the measurements and the estimates. Many implementations use simple kinematic process models to provide state estimates. The KF has been continually expanded, modified, and re-explained in the past 50 years. A good introduction to Kalman filtering methods is given in [88].

\section{The extended Kalman filter for nonlinear systems}

The Extended Kalman filter (EKF) is a generalization of the Kalman filter to deal with nonlinear systems. It linearizes the system at the current estimated state, then analytically propagates a Gaussian random variable through the linearized transition equations. Although this process can be complicated, the EKF is the de facto standard for many estimation applications. It has been widely applied to AUVs, both as a real-time estimator (e.g., [92]) and as a postprocessing filter-smoother (e.g., [49]). It is also used in many of the single-range LBL systems mentioned in section 0.2.1.

\section{Sigma point Kalman filters for nonlinear systems}

Julier and Uhlmann introduced the unscented transform (UT) as a new approach to pass important statistics of a random variable through a general nonlinear transformation [93-95]. It was extended to the unscented Kalman filter (UKF) for filtering nonlinear systems in $[96,97]$. It has since been shown to outperform the extended Kalman filter (EKF) in many situations [96-98].

The UKF has been classified as an example of sigma point filtering [99-101]. The sigma point approach uses a weighted, deterministic sampling scheme to propagate estimated error through nonlinear system dynamics. This is essentially a formalism to perform weighted statistical linear regression (WSLR) as a random variable passes through many general transformations. It provides improved performance in highly 
nonlinear systems, and does not require analytical derivation of Jacobians for the process model. This makes it attractive in a 'black-box' sense, and should simplify its application to a coupled nonlinear system like an underwater vehicle.

\section{Handling delayed measurements}

The simplest way to deal with a measurement delay is simply to ignore it and fuse the measurement as if it is an observation of the system state at the current instant. This is the standard approach, but if the delay is significant it will produce inaccurate estimates. A brute force approach would recalculate the state estimates over the entire delay interval after the measurement arrives. This would provide accurate estimates, but at increased computational cost. Depending on the length of the delay, this approach can become impractical or untractable. Here we will review some more principled methods to account for the delay.

State augmentation is a standard method to handle situations where the current state of a system is correlated to another variable-including its past state. This has been applied to linear systems with single or multiple delays (e.g., [102]). Used carefully, state augmentation can enable a KF to handle delayed measurements.

Leonard \& Rikoski use an EKF with augmented states representing the past trajectory of a mobile robot in [103]. These augmented states are used in feature initialization for concurrent mapping and localization-enabling the robot to use data from multiple timesteps for improved data association and decision making. In [104], Rikoski applies this approach to handle time-of-flight measurements in LBL acoustic positioning for an underwater vehicle ${ }^{1}$. The augmented state includes the full state at the instant the robot interrogates the net, as well as the full state at the instant it receives the reply from each transponder.

Ridao, et al.use a bounded circular buffer of past poses ${ }^{2}$ to fuse USBL position

\footnotetext{
${ }^{1}$ Section 3.6 explores a similar application-formulated independently of Rikoski's work-using the model-driven delayed measurement fusion approach presented in this chapter for LBL-aided DR.

${ }^{2}$ Since the delayed USBL measurements only relate to the position of the remotely operated vehicle (ROV) and not its velocity, Ridao, et al.use the 4-element pose vector for each augmented state, instead of the full 8-element state vector. This reduces the dimension of the filter-and saves some computation cost-but still uses more resources than strictly necessary.
} 
measurements with DR based on an attitude and heading reference system (AHRS) and a DVL in [105]. They assume that the clocks of the USBL system and the vehicle are synchronized ${ }^{3}$, and validate the method using a tethered ROV. This approach has the advantage that it imposes an upper limit on the growth of the state and filter dimension. To ensure the appropriate state will be in the buffer when the delayed measurement arrives, the buffer must include all past poses up to the maximum delay length-this increases the dimension of the filter and the computation cost. For this approach to work, the bounded circular buffer must always contain more past vehicle poses than the fusion step of the filter requires-so by definition, this implementation uses more resources than necessary.

To reduce this extra cost, Challa suggests a variable dimension augmented state $\mathrm{KF}$ in [102]. Instead of storing all the past states over the entire delay interval, this filter adaptively augments only the essential past states-the ones that the delayed measurement will observe ${ }^{4}$. This results in a more efficient filter with comparable estimation accuracy.

Approaching the problem from a different direction, some researchers use a standard KF formulation during the delay interval, then calculate a different correction term when fusing the delayed measurement.

Hilton, et al. apply this to tracking problems with time-delayed measurements in [106]. Assuming constant-velocity tracking, they back-propogate the state estimate through linearized system dynamics to the instant the delayed measurement was valid. They then minimize the trace of the covariance to solve for a modified Kalman gain. Simulations showed that this approach outperformed several ad-hoc methods, and nearly recovered the performance of a filter with no delay in its measurements.

In $[22,78]$, Farrell uses an error state EKF for sensor fusion aboard an AUVaiding an inertial navigation system (INS) with velocity measurements from a DVL,

\footnotetext{
${ }^{3}$ This restriction is not necessary if the USBL system can report the time it is interrogated, see section 3.5.

${ }^{4}$ Although Challa's work does not investigate measurements that are composite observations of the state at two distinct instants, the variable dimension augmented state KF has a similar delay treatment to Rikoski [104]. This variable dimension approach is also similar to the work by van der Merwe, et al., which is discussed later in this section.
} 
heading measurements from a magnetometer, depth measurements from a pressure sensor, and two-way travel time measurements from four LBL transponders ${ }^{5}$. The measurement delay compensation uses a wait-and-correct strategy. Any time the vehicle interrogates the LBL net, the error state accumulates until the end of that LBL measurement cycle. In the meantime, navigation relies entirely on the INSmeasurements from other aiding sensors are not fused during the delay interval. This effectively reduces the aiding rate to the rate of the slowest sensor-including measurement delays. While this approach may be suitable for a system with high quality inertial navigation, it may not perform well on a system with a low-grade INS, and is not directly applicable to any system without inertial sensing.

Alexander proposed an approach in [107] where a correction term was added to the estimates of a linear KF once the delayed measurement was available. The covariance, however, was updated early, when the measurement should have been valid-this resulted in sub-optimal fusion of any measurements during the delay interval. Larsen, et al., reformulated this approach in [108] to make it easier to implement. That study also suggested a two-filter method to optimally fuse all measurements, with or without delay. This modified two filter method achieved the same low estimation error as brute-force recalculation, but at reduced computational cost. It was applied to vision-aided navigation for an autonomous unmanned ground vehicle (UGV) in [109].

van der Merwe, et al. adapted Larsen's two-filter method to nonlinear systems using a sigma point Kalman filter (SPKF) with augmented states instead of separate filters $^{6}$ [99-101], bringing the 'filter and correct' approaches back to state augmentation. This delayed-state SPKF was applied to navigate an autonomous helicopter unmanned aerial vehicle (UAV) with fast dynamics and a slow GPS receiver. Although

\footnotetext{
${ }^{5}$ The LBL measurement equation in $[22,78]$ deals with the fact that a two-way travel time is a composite observation of the vehicle state at two distinct instants by back-propogating the state estimate at the reply instant through linearized vehicle kinematics to the interrogation instant.

${ }^{6}$ Although delay compensation can be achieved with state augmentation, care must be taken when initializing or updating the augmented states. The method given in equations 5.63 and 5.64 of [99] produces a covariance matrix with four identical blocks-this is guaranteed to be singular. The model-driven delayed measurement fusion method presented in this chapter avoids this issue by using appropriate time-varying conditional process models to initialize and update the augmented states.
} 
the delay was only on the order of $50 \mathrm{~ms}$, compensating for it reduced estimation error in position, velocity, and attitude.

\subsubsection{Contributions}

The method proposed in this chapter is an efficient, flexible, and mathematically principled approach to delayed measurement fusion. It is based on state augmentationadding only the minimal amount of extra memory necessary to deal with delays. It works entirely within the KF framework-using time-varying conditional models to manage the augmented states-separating the user from any external or ad hoc adjustments to the state estimate or filter covariance.

Table 3.1 compares the model-driven approach to other related work in the literature. The main points are the minimal increase in dimension and the ability to work entirely within a single KF. It should work with any ${ }^{7}$ kind of KF, and is demonstrated here using the classical KF for linear systems and the SPKF for nonlinear systems.

The previous literature that includes delay compensation can be loosely divided into methods using state augmentation, and methods that use an external process to back-propogate or wait-and-correct. For a filter with an original state dimension $N$, the state augmentation approaches use a filter of dimension $2 N$ to handle the delay ${ }^{8}$. The external processes in the other methods also have calculations on the order of $N$, for an effective dimension $\sim 2 N$. The model-driven approach proposed here, on the other hand, produces a filter with dimension $N+M$, where $M$ is the dimension of the delayed measurement, and usually $M<N$.

Many of these approaches can be extened to deal with multiple delayed measurements. This ability comes with additional increase in dimension, associated with $D$, the number of delayed measurements. The numbers in Table 3.1 denote this additional cost only for work where support for multiple delayed measurements was demonstrated.

\footnotetext{
${ }^{7}$ including an EKF, although that has not been demonstrated here and remains for future work

${ }^{8}$ Ridao uses a filter with dimension $N+L P$, where $L$ is the maximum delay length, and $P$ is the dimension of a reduced state (the pose). In practice, the maximum delay length may be very large, so even though $P<N, L P$ is likely greater than $N$, and the total filter dimension is $N+L P \gg 2 N$.
} 

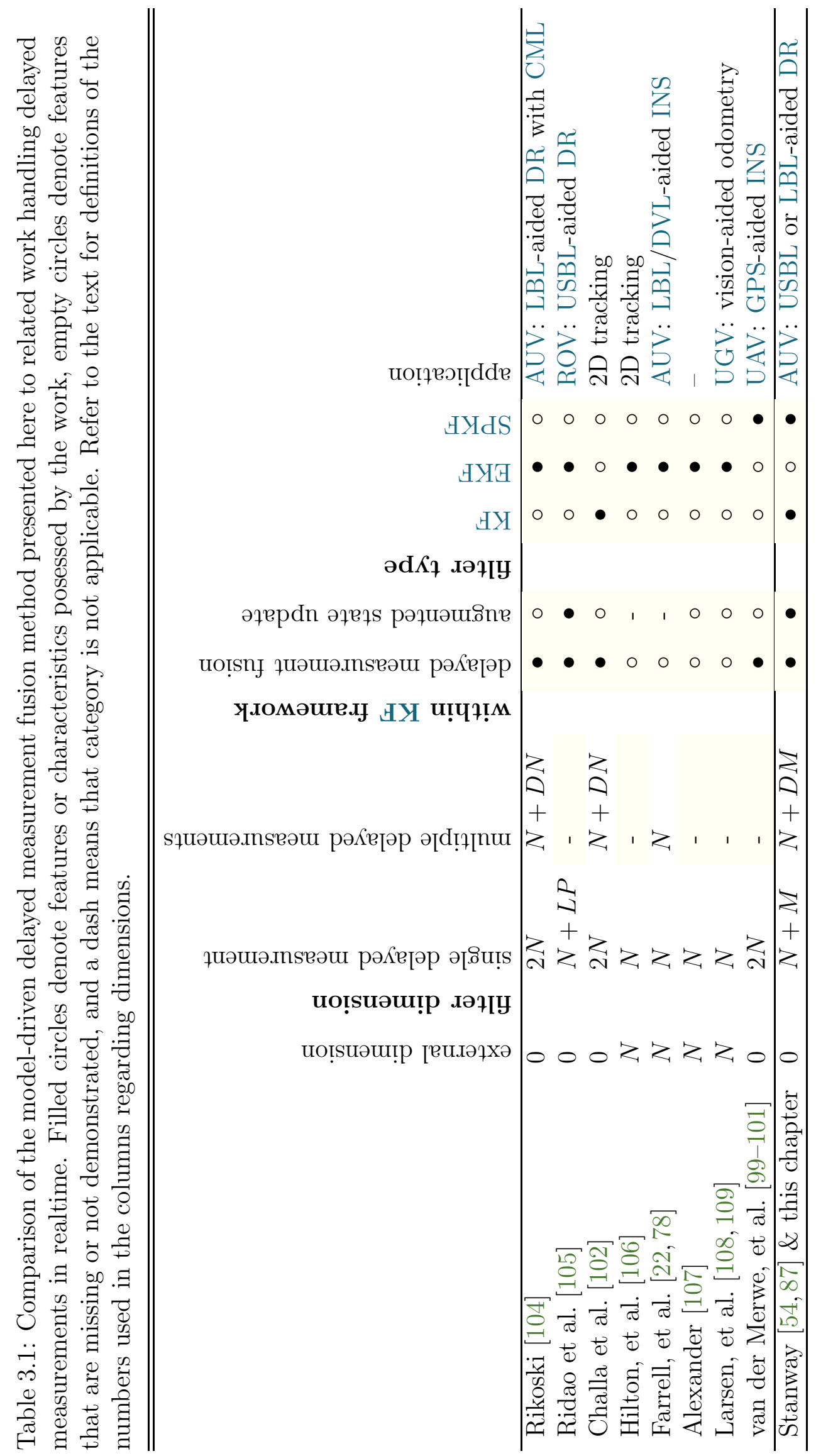
The methods relying on external processes have the additional downside that they are not integrated into the filter structure, and therefore less portable to other applications and other types of KF (e.g. SPKFs for nonlinear systems.)

The approach presented here handles delayed measurements in a mathematically principled way with a modest increase in filter dimension (and associated computational complexity). Its flexibility allows it to be applied to linear or nonlinear systems, in synchronous or asynchronous filters. The next few sections will show how the model-driven approach makes delay treatment simple for the user reduces error and uncertainty in state estimates.

\subsection{Types of measurement delay}

Any single measurement may be subject to several distinct delays of different types: physical, communications, hardware/firmware, and processing.

Physical delays are governed by the laws of nature. One example is the travel time delay for an electromagnetic or acoustic wave. Little can be done to reduce physical delays without changing operations-minimizing the distance to acoustic beacons, for example. These delays are negligible in some cases (e.g. GPS localization of an automobile), but significant in others (e.g. long-range LBL localization of an underwater vehicle).

Communications delays are primarily due to network latency. This means they overlap a bit with physical delays, but they also include delays from dropped packets or incomplete feedback.

Hardware and firmware delays are often included to protect equipment and ensure reliable operation. Examples include the blanking time an acoustic transceiver waits before listening for a return, and the turn around time a transponder waits before sending a reply. These delays usually have recommended values and limited adjustability.

Finally, processing delays are dictated by the complexity of the problem and the algorithm used to solve it. Much can (and has) been done to minimize this type of 
delay in modern systems, and computers are only getting faster. However, embedded devices with limited processing power can still impose non-negligible delays. This was the case in the first application of a SPKF to a delayed measurement problem [100, 101].

\subsection{Model-driven delayed measurement fusion}

This section proposes a new, entirely model-based, approach to delayed measurement fusion. This flexible approach can treat multiple delayed measurements with lengths that vary in time-it can even handle measurements with multiple delays. Perhaps most importantly, this approach handles delayed measurements in a mathematically principled fashion without changing the underlying structure of the KF framework.

This section presents the theory behind model-driven delayed measurement fusion. We begin by reviewing the underlying KF framework and the key concepts behind it. Then we carefully define the nomenclature and notation for the timeline of delayed and undelayed measurements. Then we show how state augmentation can be used to store the necessary information for delayed measurement fusion. Then we show how conditional, time-varying process and measurement models can be used to manage the augmented states and delayed measurements without changing the KF framework. The next section will walk through an example application using a damped harmonic oscillator.

\subsubsection{Timeline and nomenclature for delayed measurements}

Before diving into the details of delayed measurement fusion, it will be helpful to take a moment and carefully define our reference frame and nomenclature. Data from several different instants of time are used, so it is important to orient ourselves and be consistent.

Consider the relevant instants in time looking back from the time of fusion, as illustrated in Figure 3-1: 


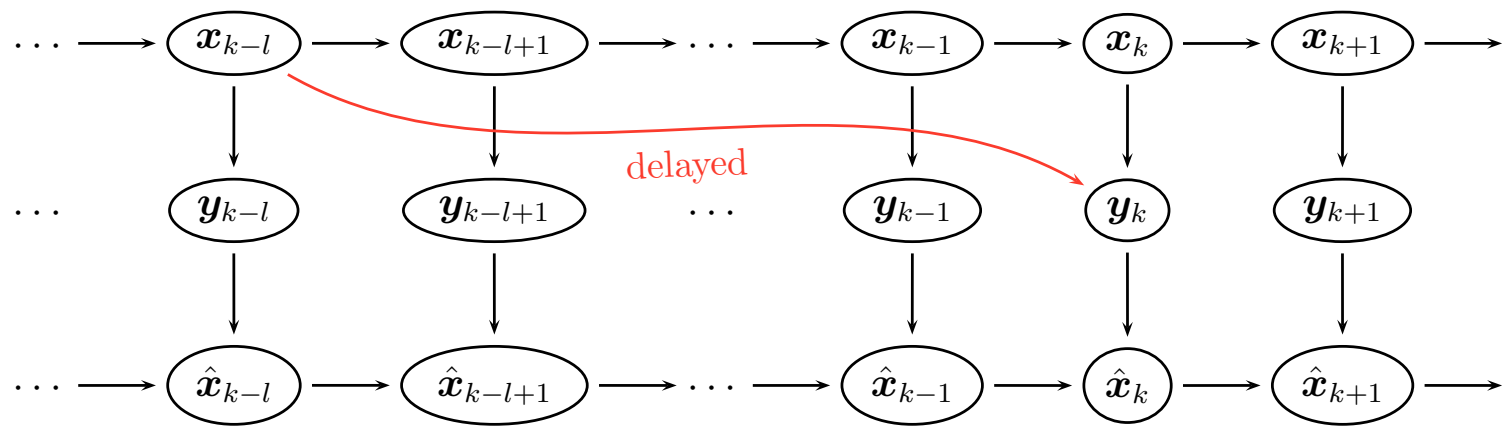

Figure 3-1: Timeline and nomenclature for delayed measurements, looking back in time from the fusion instant $k$. A delayed measurement with latency of $l$ timesteps is an observation of the system state at time $k-l$. Other measurements may be available to the filter in the intermediate period between timestep $k-l$ and the fusion timestep $k$, when the delayed measurement becomes available. In some situations, more delayed measurements related to the same system state at $k-l$ may arrive after even longer delays.

trigger timestep $k-l$

The delayed measurement is an observation of the state of the system at this instant. The measurement will not be available to the filter until $l$ timesteps later.

intermediate timestep $k-l+m$

Other measurements (especially from other sensors) may be available in the interval between the time the measurement is valid and the time it is available to the filter.

fusion timestep $k$

The delayed measurement, an observation of the past state of the system $\boldsymbol{x}_{k-l}$, becomes available to the filter. The information in this measurement should be used to update the current estimate of the state: $\hat{\boldsymbol{x}}_{k} \rightarrow \hat{\boldsymbol{x}}_{k+1}$.

afterward timestep $k+n$

In some situations ${ }^{9}$, there may be more observations of the previous system state. These should be handled in the same way as the first delayed measurement.

\footnotetext{
${ }^{9}$ e.g., the LBL-aided DR example in section 3.6
} 
Alternately, looking forward from the instant that the measurement observes the system, the relevant timestps are $k, k+m, k+l, k+l+n$. The dummy indices $m, n$ will change as the system and filter state evolve. They help provide precise notation, but may never be explicitly tracked or used by the filter. The latency $l$ may not be constant, or even explicitly known.

With this nomenclature in mind, we can discuss the KF framework and propose a specific method for dealing with delayed measurements without altering that framework.

\subsubsection{The Kalman filter framework}

Our goal is to effectively and efficiently fuse delayed measurements into the current state estimate of a dynamic system. To encourage understanding and simplify exposition, we will begin with a linear discrete state space model (DSSM).

$$
\begin{aligned}
\boldsymbol{x}_{k+1} & =\mathbf{A}_{k} \boldsymbol{x}_{k}+\mathbf{B}_{k} \boldsymbol{u}_{k}+\mathbf{G}_{k} \boldsymbol{v}_{k} \\
\boldsymbol{y}_{k} & =\mathbf{C}_{k} \boldsymbol{x}_{k}+\boldsymbol{w}_{k}
\end{aligned}
$$

The system state $\boldsymbol{x}_{k}$ is driven by the known control input $\boldsymbol{u}_{k}$ and the unknown process noise $\boldsymbol{v}_{k} \sim \mathcal{N}\left(0, \mathbf{Q}_{k}\right)$. Observations $\boldsymbol{y}_{k}$ are a function of the current state, with random measurement noise $\boldsymbol{w}_{k} \sim \mathcal{N}\left(0, \mathbf{R}_{k}\right)$.

Given these observations, the Kalman filter (KF) provides an optimal estimate of the system state. Many different derivations exist, see for example [88,91].

The KF is often presented in two steps: predict and update. The prediction step computes a priori estimates of the state, its covariance, and the observation:

$$
\begin{aligned}
\hat{\boldsymbol{x}}_{k+1}^{-} & =\mathbf{A}_{k} \hat{\boldsymbol{x}}_{k}^{+}+\mathbf{B}_{k} \boldsymbol{u}_{k} \\
\mathbf{P}_{k+1}^{-} & =\mathbf{A}_{k} \mathbf{P}_{k}^{+} \mathbf{A}_{k}^{T}+\mathbf{Q}_{k} \\
\hat{\boldsymbol{y}}_{k+1} & =\mathbf{C}_{k} \hat{\boldsymbol{x}}_{k+1}^{-}
\end{aligned}
$$




\section{Adjusting the prediction: innovations}

The first key concept of KFs is the innovation-this is simply the difference between predicted and observed measurements of the system state:

$$
\tilde{\boldsymbol{y}}_{k}=\boldsymbol{y}_{k}-\hat{\boldsymbol{y}}_{k}
$$

this is also called the measurement residual. In the KF framework, the filter doesn't really care about the exact value of any given measurement-it really only wants to know how far off the prediction was-this is the key that will enable us to handle measurements with delays. The update step of the KF applies the optimal gain to the innovation to compute a posteriori estimates of the state and covariance:

$$
\begin{aligned}
\hat{\boldsymbol{x}}_{k}^{+} & =\hat{\boldsymbol{x}}_{k}+\mathbf{K}_{k}\left(\boldsymbol{y}_{k}-\hat{\boldsymbol{y}}_{k}\right) \\
\mathbf{P}_{k}^{+} & =\left(\mathbf{I}-\mathbf{K}_{k} \mathbf{C}_{k}\right) \mathbf{P}_{k}^{-},
\end{aligned}
$$

which brings us to the second key concept: the optimal Kalman gain.

\section{The optimal Kalman gain: a ratio of uncertainties}

Given a state estimate with some quantified uncertainty and an observation of that state with some other uncertainty, an optimal approach to fuse these two bits of information is to weight them by the ratio of uncertainties. This is the approach used in the $\mathrm{KF}$ framework.

A minimum mean-squared error (MMSE) derivation of the KF (e.g., [99]) gives the Kalman gain [97, 101]:

$$
\mathbf{K}_{k}=\mathbf{P}_{\tilde{\boldsymbol{x}}_{k} \tilde{\boldsymbol{y}}_{k}}^{-}\left(\mathbf{P}_{\tilde{\boldsymbol{y}}_{k} \tilde{\boldsymbol{y}}_{k}}^{-}\right)^{-1}
$$

where $\tilde{\boldsymbol{x}}_{k}^{-}, \tilde{\boldsymbol{y}}_{k}^{-}$are the residuals of the a priori estimates of the state and the observation, and their (cross-) covariances are $\mathbf{P}_{\tilde{\boldsymbol{x}}_{k} \tilde{y}_{k}}^{-}, \mathbf{P}_{\tilde{\boldsymbol{y}}_{k} \tilde{\boldsymbol{y}}_{k}}^{\bar{n}}$. 


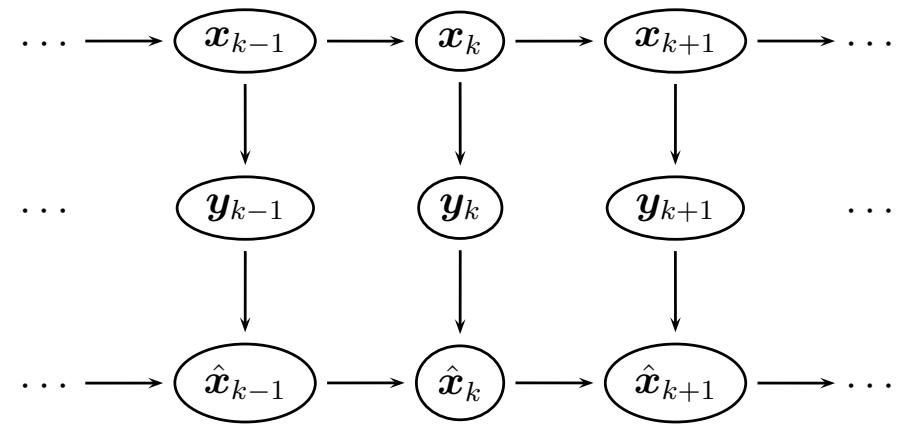

Figure 3-2: Simple graph representation of a KF. The unobserved true state $\boldsymbol{x}$ is a Markov process and the noisy measurements $\boldsymbol{y}$ are the observations of a hidden Markov model. The KF used these observations to estimate the state $\hat{\boldsymbol{x}}$.

This is equivalent to the standard form given in most texts (e.g., [88]):

$$
\mathbf{K}_{k}=\mathbf{P}_{k}^{-} \mathbf{C}_{k}^{T}\left(\mathbf{C}_{k} \mathbf{P}_{k}^{-} \mathbf{C}_{k}^{T}+\mathbf{R}_{k}\right)^{-1}
$$

In (3.7a), we see that the key to correctly fusing any measurement is to have an estimate of its cross-covariance with the state. With a delayed measurement, you simply need to use the correct cross-covariance.

\section{Implications of the Markov Assumption}

One basic assumption of the $\mathrm{KF}$ is that the system state is a Markov process-that is, a process with no memory. The standard KF can be viewed as an estimator operating on the observations from a hidden Markov model (HMM) (Figure 3-2). Each measurement is an observation of the current system state, and by the Markov assumption:

conditional on the current state, the future states of the system are independent of all the past states.

This poses a problem for fusing delayed measurements, where some sort of memory is required to properly relate the delayed measurement to a past system state. A delayed measurement violates the Markov assumption. One way to get around this is to augment the filter state vector with extra variables to maintain information about a specific past state. 


\subsubsection{Maintaining the delayed innovation and cross-covariance: state augmentation}

State augmentation expands the filter dimension to include extra information (in this case, related to a specific system state in the past.) This additional information $\boldsymbol{a}$ is concatenated to the original state $\boldsymbol{x}$ to produce the augmented state vector $\boldsymbol{x}^{a}$ :

$$
\begin{aligned}
\boldsymbol{x}^{a} & =\left[\begin{array}{l}
\boldsymbol{x} \\
\boldsymbol{a}
\end{array}\right] \\
& =\left[\begin{array}{lllllllll}
x_{1} & x_{2} & \cdots & x_{M} & a_{1} & a_{2} & \cdots & a_{L}
\end{array}\right]^{T}
\end{aligned}
$$

van der Merwe, et. al. used this approach in [99-101] to fuse latent GPS measurements of the position of a helicopter UAV. In that study, the augmented states included a full copy of the original state vector, here we employ a subtly different approach.

\section{Delayed innovation}

We specifically use a priori estimates of future measurements as augmented states. At the trigger instant:

$$
\hat{\boldsymbol{x}}_{k}^{a}=\left[\begin{array}{c}
\hat{\boldsymbol{x}}_{k} \\
\hat{\boldsymbol{y}}_{k+m}^{-}
\end{array}\right]
$$

or at the fusion instant

$$
\hat{\boldsymbol{x}}_{k}^{a}=\left[\begin{array}{c}
\hat{\boldsymbol{x}}_{k} \\
\hat{\boldsymbol{y}}_{k-l}^{-}
\end{array}\right]
$$

This maintains all the information necessary to fuse the delayed measurement later. And, it keeps the filter dimension small ${ }^{10}$-this is important for realtime operations on

\footnotetext{
${ }^{10}$ Using a priori estimates of future measurements as the augmented states ensures a near-minimal increase in filter dimension. In some specific cases (e.g., overconstrained LBL with more than 4 transponders) the dimension could be mildly decreased further. The difference is small compared
} 
a wide variety of platforms, or if the $\mathrm{KF}$ is the kernel of a more intensive process (e.g., to update the particles in a particle filter). This approach also pre-computes so that the innovation can be calculated immediately and the processing delay of the filter itself is minimized. A more subtle advantage is that it helps ensure that the filter remains stable-introducing the measurement noise into the augmented covariance (detailed in the next section) helps keep it from becoming singular.

\section{Cross-covariances}

The full augmented covariance matrix $\mathbf{P}_{k}^{a-}$ is:

$$
\mathbf{P}_{k}^{a-}=\left[\begin{array}{cc}
\mathbf{P}_{\tilde{\boldsymbol{x}}_{k}^{-} \tilde{\boldsymbol{x}}_{k}^{-}} & \mathbf{P}_{\tilde{\boldsymbol{x}}_{k}^{-} \tilde{\boldsymbol{y}}_{k+m}^{-}} \\
\mathbf{P}_{\tilde{\boldsymbol{y}}_{k+m}^{-} \tilde{\boldsymbol{x}}_{k}^{-}} & \mathbf{P}_{\tilde{\boldsymbol{y}}_{k+m}^{-} \tilde{\boldsymbol{y}}_{k+m}^{-}}
\end{array}\right]
$$

It contains the original covariance matrix $\mathbf{P}_{\tilde{\boldsymbol{x}}_{k} \tilde{\boldsymbol{x}}_{k}}$, the covariance of the delayed innovation $\mathbf{P}_{\tilde{\boldsymbol{y}}_{k+m}^{-}} \tilde{\boldsymbol{y}}_{k+m}^{-}$, and the cross-covariances between them $\mathbf{P}_{\tilde{\boldsymbol{x}}_{k}^{-} \tilde{\boldsymbol{y}}_{k+m}^{-}}=\mathbf{P}_{\tilde{\boldsymbol{x}}_{k}^{-} \tilde{\boldsymbol{y}}_{k+m}^{-}}^{T}$. Recalling (3.7a), we see that the augmented covariance matrix maintains all the information necessary to fuse the delayed measurement.

\subsubsection{Driving the new filter: time-variant conditional process and measurement models}

As mentioned earlier, an augmented state means process and measurement models need to be adjusted anyway-why not use this to our advantage and define these models to handle the augmented states and delayed measurements without any ad-hoc adjustments to the filter?

\section{Trigger process model}

The filter needs a way to choose between different process models. In the examples of this chapter, the trigger is obvious and can be provided by the system-in other

to storing the full past state as in [99-101], or multiple past states as in [105] or a pose graph implementation. 
cases, one might have to use additional augmented states to estimate the time of the trigger condition.

The trigger process model is always the same. The first part is simply the original process model. The second part is the measurement model for the (expected) delayed measurement. This is flexible enough to work with many kinds of systems, but specific enough to ensure both the original and augmented states are updated properly.

The measurement error covariance matrix should be $\operatorname{added}^{11}$ to the augmented state covariance, just as if it were a regular measurement.

\section{Loosely-Coupled Measurements}

In this case, the delayed measurement is some direct (linear) function of state(s). An example would be a USBL system that provides a fully-computed geographic position fix.

The augmented state is the a priori estimate of the delayed measurement, so the delayed innovation is straightforwarrd:

$$
\begin{aligned}
\tilde{\boldsymbol{y}}_{k} & =\boldsymbol{y}_{k}-\hat{\boldsymbol{y}}_{k}^{-} \\
& =\boldsymbol{y}_{k}-\mathbf{C}_{k}^{\text {select }} \hat{\boldsymbol{x}}_{k}^{a}-
\end{aligned}
$$

where $\mathbf{C}_{k}^{\text {select }}$ is simply a matrix of ones and zeros that selects the appropriate entry in the augmented state vector.

\section{Tightly-Coupled Measurements}

This approach involves a more complex measurement model. For example, the full interrogation-response time for a particular transponder in an LBL net.

In this case, the delayed measurement may combine several augmented states (i.e., from multiple delays) or may combine delayed and undelayed information:

$$
\tilde{\boldsymbol{y}}_{k}=\boldsymbol{y}_{k}-\left(\mathbf{C}_{k}^{\text {select }} \boldsymbol{x}_{k}^{a-}+h^{\text {undelayed }}\left(\boldsymbol{x}_{k}^{a-}\right)\right)
$$

\footnotetext{
${ }^{11}$ or equivalently treated, if the measurement noise is not strictly additive
} 
We will revisit this kind of measurement in section 3.6.

\subsection{A canonical example: the damped harmonic oscillator}

We introduce the model-driven delayed measurement fusion approach using a simple one-dimensional system, showing the natural progression from the standard KF to our new delayed measurement filter. Along the way, we use simulations to study the behavior of a KF without delayed measurements, a KF with delayed measurements that ignores the delay, and the new filter that compensates for measurement delays. We also use this example to discuss an alternative ad-hoc approach to compensate for measurement latency.

\subsubsection{System Dynamics}

The dynamics of a generalized damped harmonic oscillator (DHO) are described by the second-order differential equation:

$$
\ddot{\xi}(t)+2 \zeta \omega_{n} \dot{\xi}(t)+\omega_{n}^{2} \xi(t)=u(t)+v(t)
$$

where $\zeta$ is the damping coefficient, $\omega_{n}$ is the natural frequency, $u(t)$ is a known control

input signal, and $v(t)$ is an unknown environmental disturbance. For this example, we will assume zero control:

$$
u(t)=0 \forall t
$$

and mutually uncorrelated additive white Gaussian process noise:

$$
v(t) \sim \mathcal{N}\left(0, \sigma_{v}\right), \quad \mathrm{E}\left[v\left(t_{1}\right) v\left(t_{2}\right)\right]=0 \forall t_{1} \neq t_{2}
$$

This simple system may not be intellectually stimulating when taken alone, but 
its simplicity makes it ideal for illustrating the progression of methods for fusing more complex types of measurement sequences with and without delay.

If the system is a point mass connected to a linear spring and damper, the generalized variable $\xi$ represents position, $\dot{\xi}$ is velocity, and $\ddot{\xi}$ is acceleration.

\subsubsection{Discrete State Space Model}

The system dynamics (3.16) are written in state space form as:

$$
\left[\begin{array}{l}
\dot{x}_{1} \\
\dot{x}_{2}
\end{array}\right]=\left[\begin{array}{cc}
0 & 1 \\
-\omega_{n}^{2} & -2 \zeta \omega_{n}
\end{array}\right]\left[\begin{array}{l}
x_{1} \\
x_{2}
\end{array}\right]+\left[\begin{array}{l}
0 \\
1
\end{array}\right] v
$$

where $x_{1}=\xi$, and $x_{2}=\dot{\xi}$.

Direct Euler integration ${ }^{12}$ gives an approximate DSSM for the dynamic process:

$$
\begin{aligned}
{\left[\begin{array}{l}
\xi_{k+1} \\
\dot{\xi}_{k+1}
\end{array}\right] } & \simeq\left[\begin{array}{cc}
1 & \Delta t \\
-\omega_{n}^{2} \Delta t & 1-2 \zeta \omega_{n} \Delta t
\end{array}\right]\left[\begin{array}{l}
\xi_{k} \\
\dot{\xi}_{k}
\end{array}\right]+\boldsymbol{v}_{k} \\
\boldsymbol{x}_{k+1} & =\mathbf{A} \boldsymbol{x}_{k}+\boldsymbol{v}_{k}
\end{aligned}
$$

This process model predicts the future state, given the current state.

\subsubsection{Full measurements}

Now assume that the system has sensors to measure its position $\xi$ and velocity $\dot{\xi}$. Both measurements are corrupted by mutually uncorrelated additive Gaussian white noise:

$$
\begin{aligned}
\boldsymbol{w} & \sim \mathcal{N}(0, \mathbf{R}), \quad \mathbf{R}=\left[\begin{array}{cc}
\sigma_{w_{1}} & 0 \\
0 & \sigma_{w_{2}}
\end{array}\right] \\
\mathrm{E}\left[\left(\boldsymbol{w}_{i}\right)\left(\boldsymbol{w}_{j}\right)^{T}\right] & =0 \forall i \neq j
\end{aligned}
$$

\footnotetext{
${ }^{12}$ For continued simplicity, we use Euler integration to move from continuous to discrete time. This is also known as a zero-order-hold approach. Alternative approaches, for example using Tustin's method (bilinear interpolation), or the exact matrix exponential, may prove valuable in future studies of delayed measurement fusion.
} 
The filter receives instantaneous measurements from both sensors at each timestep. This direct ${ }^{13}$ measurement model is:

$$
\begin{aligned}
{\left[\begin{array}{l}
y_{1} \\
y_{2}
\end{array}\right] } & =\left[\begin{array}{ll}
1 & 0 \\
0 & 1
\end{array}\right]\left[\begin{array}{l}
\xi \\
\dot{\xi}
\end{array}\right]+\left[\begin{array}{l}
w_{1} \\
w_{2}
\end{array}\right] \\
\boldsymbol{y}_{k} & =\mathbf{C} \boldsymbol{x}_{k}+\boldsymbol{w}_{k},
\end{aligned}
$$

\subsubsection{Fusing measurements with different sampling periods}

In many practical applications, engineers need to fuse multiple types of measurements, each with different sampling frequencies. To enable this, we must make a slight modification to the standard KF problem presented above.

The physical system has not changed, so the process model (3.20) stays the same. However, the measurement system has changed, so the measurement model (3.22b) must also change to reflect this.

\section{Measurement Selection Matrix}

In a linear (or linearized) system, we can simply pre-multiply the measurement matrix by a selection matrix:

$$
\boldsymbol{y}_{k}=\mathbf{C}_{k}^{\text {select }} \mathbf{C} \boldsymbol{x}_{k}+\boldsymbol{w}_{k}
$$

The selection matrix is a matrix of ones and zeros. It varies in time so that (3.23) generates the appropriate a priori measurement estimates to compare with the actual measurements.

For example, at discrete time instant $k$, the velocity sensor provides a measurement, but the position sensor does not. In this case, the selection matrix would

\footnotetext{
${ }^{13}$ Again, a direct measurement model may not be the most intellectually stimulating, but it serves our purposes well here. We will consider a more complex type of measurements in the LBL-aided DR example in section 3.6.
} 
be:

$$
\mathbf{C}_{k}^{\text {select }}=\left[\begin{array}{ll}
0 & 1
\end{array}\right]
$$

so that the measurement model (3.23) would expand to:

$$
\boldsymbol{y}_{k}=\left[\begin{array}{ll}
0 & 1
\end{array}\right]\left[\begin{array}{ll}
1 & 0 \\
0 & 1
\end{array}\right]\left[\begin{array}{l}
\xi \\
\dot{\xi}
\end{array}\right]+\left[\begin{array}{l}
w_{1} \\
w_{2}
\end{array}\right]
$$

and the measurement would be:

$$
\boldsymbol{y}_{k}=\dot{\xi}+w_{2}
$$

If both position and velocity measurements are available at the next timestep, $k+1$, the selection matrix would become the identity matrix, $\mathbf{C}_{k+1}^{\text {select }}=\mathbf{I}_{2 \times 2}$.

\section{Time-Variant Measurement Models}

The selection matrix approach presented above works well for linear (or linearized) measurement models, but must be generalized for nonlinear models. This actually turns out to be very simple.

Consider (3.23) again. The selection matrix and measurement matrix can be combined into a single, time-varying measurement matrix:

$$
\mathbf{C}_{k} \equiv \mathbf{C}_{k}^{\text {select }} \mathbf{C}
$$

For our example above:

$$
\begin{aligned}
\mathbf{C}_{k} & =\left[\begin{array}{ll}
0 & 1
\end{array}\right]\left[\begin{array}{ll}
1 & 0 \\
0 & 1
\end{array}\right] \\
& =\left[\begin{array}{ll}
0 & 1
\end{array}\right]
\end{aligned}
$$


Table 3.2: Simulation parameters used in damped harmonic oscillator example.

\begin{tabular}{c|c|c|c|c|c|c|c|c}
\hline $\begin{array}{c}\omega_{n} \\
\mathrm{rad} / \mathrm{s}\end{array}$ & $\begin{array}{c}\zeta \\
-\end{array}$ & $\begin{array}{c}\sigma_{\boldsymbol{v}_{1}} \\
\mathrm{~m}\end{array}$ & $\begin{array}{c}\sigma_{\boldsymbol{v}_{2}} \\
\mathrm{~m} / \mathrm{s}\end{array}$ & $\begin{array}{c}\sigma_{\boldsymbol{w}_{1}} \\
\mathrm{~m}\end{array}$ & $\begin{array}{c}\sigma_{\boldsymbol{w}_{2}} \\
\mathrm{~m} / \mathrm{s}\end{array}$ & $\begin{array}{c}T_{\boldsymbol{x}_{1}} \\
\mathrm{~s}\end{array}$ & $\begin{array}{c}T_{\boldsymbol{x}_{2}} \\
\mathrm{~s}\end{array}$ & $\begin{array}{c}l \\
\mathrm{~s}\end{array}$ \\
\hline \hline 1 & 0.1 & $10^{-6}$ & $10^{-2}$ & 0.1 & 0.1 & 0.42 & 0.01 & 0.40 \\
\hline
\end{tabular}

Any linear, time-variant measurement process can be described using a selection matrix or a time-variant measurement matrix. A general, nonlinear, time-variant measurement process requires general, nonlinear, time-variant measurement models. The selection matrix is useful for introduction to the concepts, but since time-variant models are more general, we'll use those for the majority of the remaining text.

\section{Simulation}

Here we study a simulation of the KF estimating the states of a DHO using position and velocity measurements with different sampling periods. The simulation parameters are given in Table 3.2. The position measurement updates at a lower rate than the velocity measurement: $f_{\boldsymbol{x}_{1}} \ll f_{\boldsymbol{x}_{2}}, T_{\boldsymbol{x}_{1}} \gg T_{\boldsymbol{x}_{2}}$. This will be our baseline simulation for comparing filters with delayed measurements in sections 3.4.5 and 3.4.6.

There are two different measurement models, and therefore two different measurement matrices:

$$
\begin{aligned}
\mathbf{C}^{\text {fast }} & =\left[\begin{array}{ll}
0 & 1
\end{array}\right] \\
\mathbf{C}^{\text {slow }} & =\left[\begin{array}{ll}
1 & 0 \\
0 & 1
\end{array}\right]
\end{aligned}
$$

such that:

$$
\begin{aligned}
\boldsymbol{y}^{\mathrm{fast}} & =\dot{\xi}+w_{2} \\
\boldsymbol{y}^{\text {slow }} & =\left[\begin{array}{l}
\xi \\
\dot{\xi}
\end{array}\right]+\left[\begin{array}{l}
w_{1} \\
w_{2}
\end{array}\right]
\end{aligned}
$$



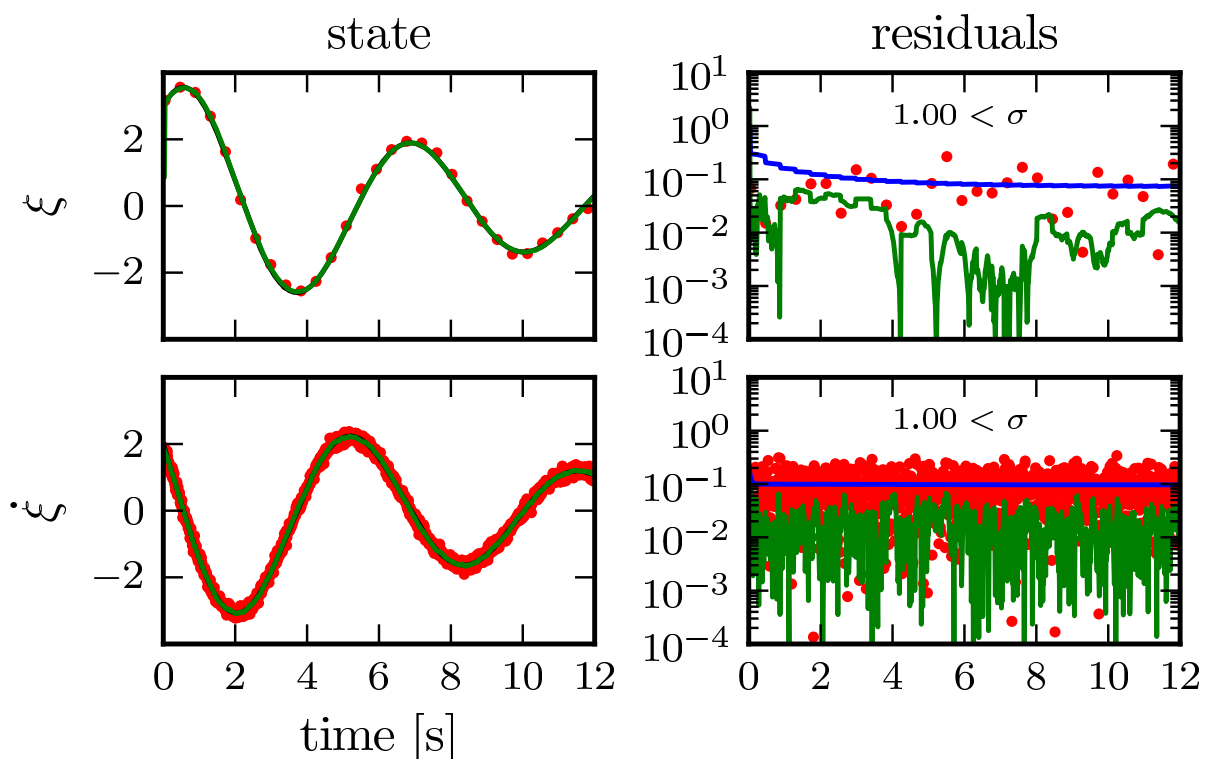

Figure 3-3: Damped harmonic oscillator simulation with a KF estimating position and velocity. The black line (covered) shows true states, red dots are the noisy measurements, and the green line shows estimates from the KF. Subplots on the right show error residuals for the measurements (red) and the KF estimates (green). The blue line is the $1 \sigma$ confidence interval of the KF estimate.

As the KF runs through each timestep, the correct time-varying conditional measurement model is chosen and the underlying machinery of the KF stays the same. Figure 3-3 shows typical filter performance for the parameter values listed in Table 3.2.

\subsubsection{Fusing delayed measurements without compensation}

Practical applications of KFs, especially in underwater navigation, often have to handle delayed measurements. It is important to deal with these measurements in a mathematically rigorous way.

Here, we consider the case where the position measurement has a finite nonzero delay, $l>0$, but it is fused as if it has no delay. In this case, (3.27d) should actually be rewritten:

$$
\boldsymbol{y}_{k}^{\text {slow }}=\left[\begin{array}{c}
\xi_{k-l} \\
\dot{\xi}_{k}
\end{array}\right]+\boldsymbol{w}_{k}
$$



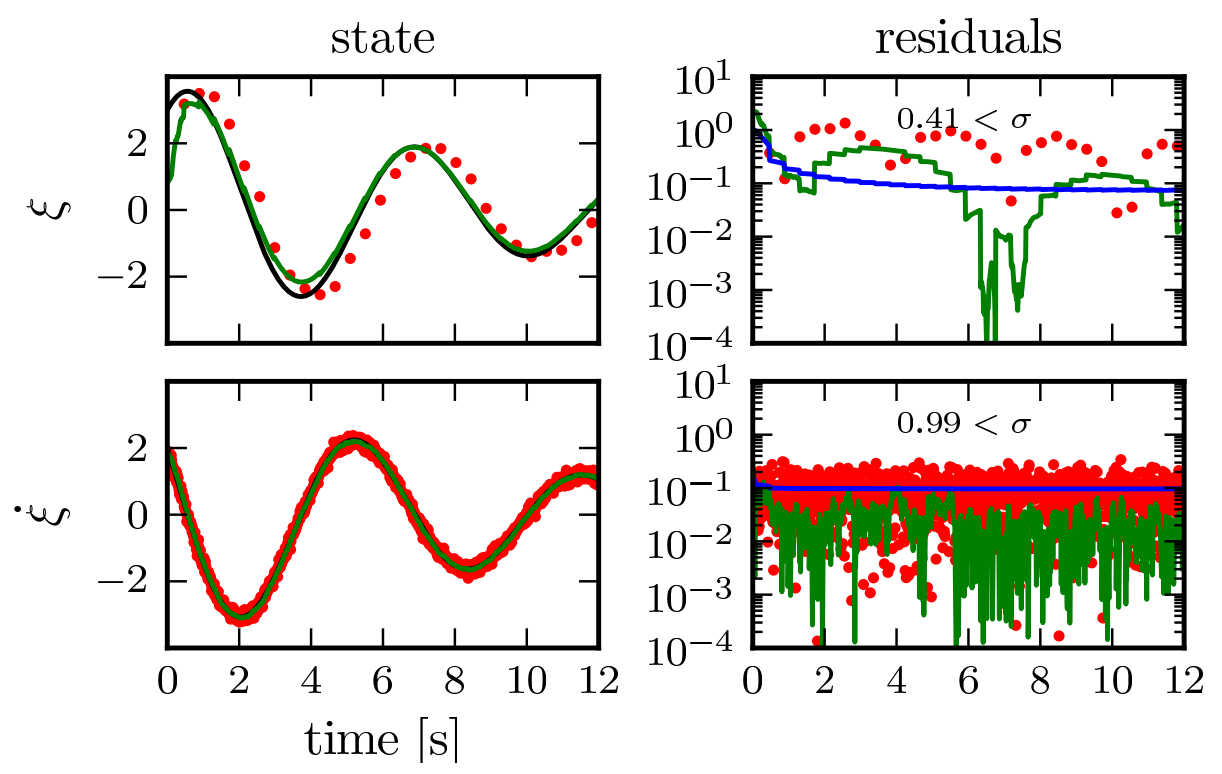

Figure 3-4: KF performance degrades considerably when position measurements are delayed, but the delay is not accounted for. The percentage of errors outside the $1 \sigma$ confidence interval is higher than expected-the filter is over-confident.

and the filter adjusted accordingly. However, the untreated filter implementation is the same as the zero delay case (Section 3.4.4), because it ignores the measurement latency.

Figure 3-4 shows the filter performance for the exact same inputs as Figure 3-3, but with the position measurements delayed by 0.4 seconds. This even uses the same values for process and measurement noise. The important thing to note is that the improper treatment of delayed measurements has made the filter overconfident-many more actual errors fall outside the $1 \sigma$ confidence bound than should. A delay almost as long as the measurement period itself may seem drastic, but this example is intended to illustrate the worst case, and then show how proper treatment of latency rectifies the issue.

\subsubsection{Fusing delayed measurements with compensation}

Finally, consider the case with $l>0$, but with proper treatment of delayed measurements using the model-driven approach proposed in section 3.3.

To be able to efficiently fuse delayed measurements, we need: 
- the innovation, and

- the cross-covariance

of the delayed measurement. We keep track of these, without altering the underlying KF machinery, by using state augmentation and time-variant conditional process and measurement models.

\section{State Augmentation}

The original system state defined in (3.20) is augmented with an a priori estimate of the delayed measurement:

$$
\hat{\boldsymbol{x}}_{k}^{a} \equiv\left[\begin{array}{c}
\hat{\boldsymbol{x}}_{k} \\
\hat{\boldsymbol{y}}_{k+m}^{-}
\end{array}\right]
$$

The integer $m$ is a dummy counter-its value need not be known ${ }^{14}$, but it is included in the notation to emphasize the fact that the extra state variable is an estimate of a measurement the filter will receive in the future.

The augmented covariance matrix is:

$$
\begin{aligned}
\mathbf{P}_{k}^{a}= & {\left[\begin{array}{cc}
\mathbf{P}_{\tilde{\boldsymbol{x}}_{k} \tilde{\boldsymbol{x}}_{k}} & \mathbf{P}_{\tilde{\boldsymbol{x}}_{k} \tilde{\boldsymbol{y}}_{k+m}} \\
\mathbf{P}_{\tilde{\boldsymbol{y}}_{k+m} \tilde{\boldsymbol{x}}_{k}} & \mathbf{P}_{\tilde{\boldsymbol{y}}_{k+m} \tilde{\boldsymbol{y}}_{k+m}}
\end{array}\right] } \\
= & {\left[\begin{array}{ccc}
\sigma_{\tilde{\xi}_{k}}^{2} & \mathbf{P}_{\tilde{\xi}_{k} \tilde{\xi}_{k}} & \mathbf{P}_{\tilde{y}_{k+m}^{-} \tilde{\xi}_{k}} \\
\mathbf{P}_{\tilde{\xi}_{k} \tilde{\xi}_{k}} & \sigma_{\tilde{\xi}}^{2} & \mathbf{P}_{\tilde{y}_{k+m}^{-} \tilde{\xi}_{k}}^{2} \\
\mathbf{P}_{\tilde{\xi}_{k} \tilde{y}_{k+m}^{-}} & \mathbf{P}_{\tilde{\xi}_{k} \tilde{y}_{k+m}^{-}} & \sigma_{\tilde{y}_{k+m}^{-}}^{2}
\end{array}\right] . }
\end{aligned}
$$

When the delayed measurement becomes available, $m=0$ and the filter will be able to calculate the innovation using the augmented state from (3.29):

$$
\tilde{y}_{k}=y_{k}-\hat{y}_{k+m}^{-}
$$

\footnotetext{
${ }^{14}$ In fact, $m$ does not even need to be kept track of. The specifics will depend on how the delayed measurement is related to the system state. In the USBL-aided DR and LBL-aided DR examples in sections 3.5 and 3.6, the navigator never needs to know $m$.
} 
and the Kalman gain using the appropriate cross-covariance from (3.30b):

$$
\mathbf{K}_{k}=\left(\mathbf{P}_{\tilde{\boldsymbol{x}}_{k} \tilde{\boldsymbol{y}}_{k+m}}^{-}\right)\left(\mathbf{P}_{\tilde{\boldsymbol{y}}_{k+m}}^{-\tilde{\boldsymbol{y}}_{k+m}}\right)^{-1}
$$

\section{Time-Variant Conditional Process Models}

Since the original state has been augmented, the process model must be modified to take this into account. A trigger process model also needs to be defined to allow the filter to update the augmented state.

The standard augmented process model leaves the a priori estimate of the delayed measurement unchanged:

$$
\begin{aligned}
\hat{\boldsymbol{x}}_{k+1}^{a-} & =\mathbf{A}_{k}^{s} \hat{\boldsymbol{x}}_{k}^{a+} \\
& =\left[\begin{array}{ccc}
1 & \Delta t & 0 \\
-\omega_{n}^{2} \Delta t & 1-2 \zeta \omega_{n} \Delta t & 0 \\
0 & 0 & 1
\end{array}\right] \hat{\boldsymbol{x}}_{k}^{a+}
\end{aligned}
$$

The trigger process model replaces the old measurement estimate with a new one based on the current state:

$$
\begin{aligned}
\hat{\boldsymbol{x}}_{k+1}^{a-} & =\mathbf{A}^{\mathrm{t}} \hat{\boldsymbol{x}}_{k}^{a+} \\
& =\left[\begin{array}{ccc}
1 & \Delta t & 0 \\
-\omega_{n}^{2} \Delta t & 1-2 \zeta \omega_{n} \Delta t & 0 \\
1 & 0 & 0
\end{array}\right] \hat{\boldsymbol{x}}_{k}^{a+}
\end{aligned}
$$

with the same process noise covariance matrix for every step:

$$
\mathbf{Q}_{k}^{a}=\left[\begin{array}{ccc}
\sigma_{\boldsymbol{v}_{1}}^{2} & 0 & 0 \\
0 & \sigma_{\boldsymbol{v}_{2}}^{2} & 0 \\
0 & 0 & 0
\end{array}\right]
$$




\section{Time-Variant Conditional Measurement Models}

The velocity sensor runs faster than the delayed position sensor-its measurement model is:

$$
\begin{aligned}
\hat{\boldsymbol{y}}_{k}^{-} & =\mathbf{C}^{\text {fast }} \hat{\boldsymbol{x}}_{k}^{a-} \\
& =\left[\begin{array}{lll}
0 & 1 & 0
\end{array}\right]\left[\begin{array}{c}
\hat{\xi}_{k}^{-} \\
\hat{\dot{\xi}}_{k}^{-} \\
\hat{y}_{k+m}^{-}
\end{array}\right]
\end{aligned}
$$

when a delayed measurement is available, an alternate measurement model selects its a priori estimate from the augmented state:

$$
\begin{aligned}
\hat{\boldsymbol{y}}_{k}^{-} & =\mathbf{C}^{\text {slow }} \hat{\boldsymbol{x}}_{k}^{a-} \\
& =\left[\begin{array}{lll}
0 & 0 & 1 \\
0 & 1 & 0
\end{array}\right]\left[\begin{array}{c}
\hat{\xi}_{k}^{-} \\
\hat{\xi}_{k}^{-} \\
\hat{y}_{k+m}^{-}
\end{array}\right]
\end{aligned}
$$

switching $\mathbf{C}_{k}$ between $\mathbf{C}^{\text {fast }}$ and $\mathbf{C}^{\text {slow }}$ based on the conditions of the available measurements ensures that:

- the appropriate innovation is calculated, and

- the correct portions of the augmented covariance matrix are used in calculating the Kalman gain.

\section{Simulation}

Figure 3-5 shows the filter performance for the exact same inputs as Figure 3-3 and Figure 3-4. This time, the position measurements are still delayed by 0.4 seconds, but we use the model-driven approach proposed in section 3.3 to compensate for the delay.

The triggers for switching between conditional process or measurement matrices are problem-specific. In this example, we have assumed that the filter has knowledge 

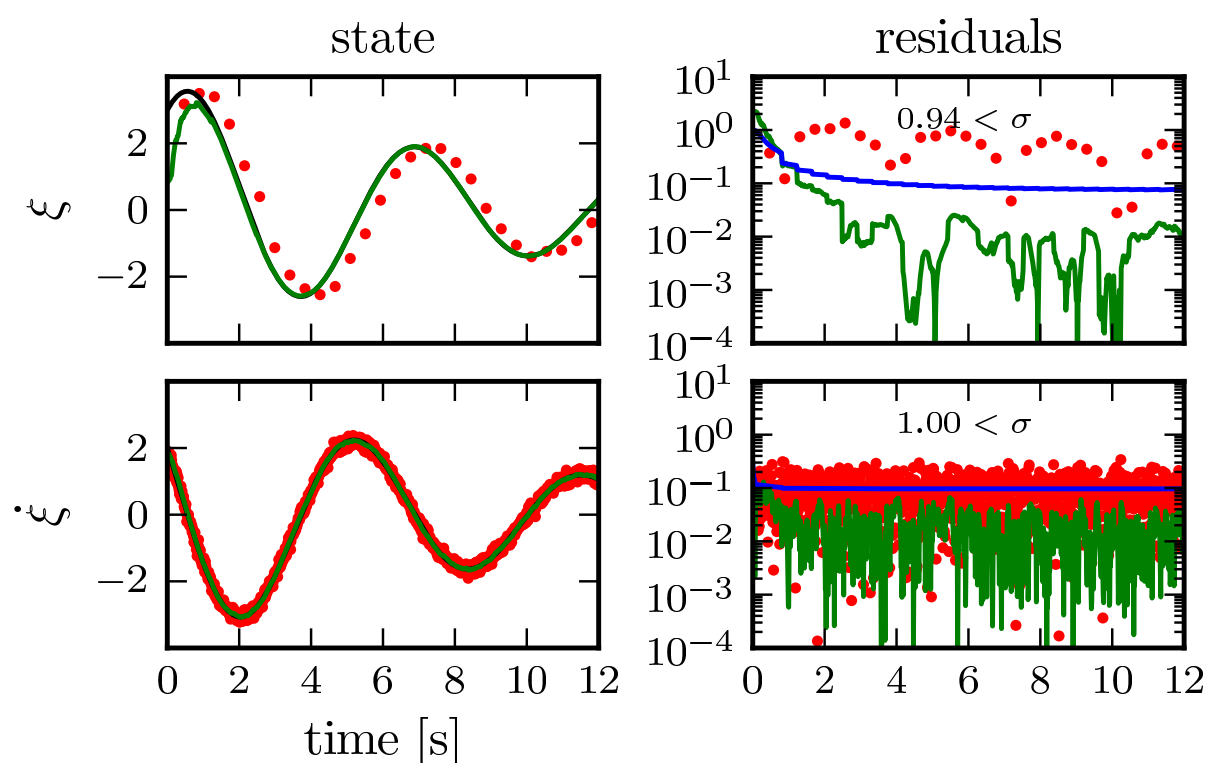

Figure 3-5: Delayed measurements are properly treated using state augmentation and conditional system models.

of which timesteps it will eventually have delayed measurements for. In practice this is often known, or can at least be estimated. In this example, we used $\mathbf{A}^{\text {trigger }}$ once every $T_{\boldsymbol{x}_{1}}=0.42$ seconds.

Note that in Figure 3-5 the filter performance is comparable to the case with no delay. By fusing the delayed measurements in a principled and mathematically rigorous way, we approach the performance of a filter fusing undelayed measurements.

\subsubsection{Some comments on alternate approaches}

This section presents alternative approaches to delayed measurement fusion. These ad hoc approaches appear to be simpler and more straightforward, especially when applied to the simple example system. However, they miss some key advantages of the more general and flexible method presented in section 3.3.

Given a system with frequent velocity measurements with no delay, and infrequent position measurements that arrive after an unknown delay, one might consider simply running the filter in the past and integrating forward in time whenever an estimate of the current state is needed. If the delayed position measurement relates to timestep $k-l$, an external process would calculate the a priori estimate of the current position 
as:

$$
\xi(t)=\xi\left(t_{k-l}\right)+\int_{t_{k-l}}^{t_{k}} \dot{\xi}(\tau) d \tau
$$

or in discrete time:

$$
\hat{\xi}_{k+1}^{-}=\hat{\xi}_{k-l}^{-}+\sum_{j=k-l}^{k} \hat{\dot{\xi}}_{j}^{+} \Delta t
$$

The external process would have to recompute the sum at each timestep in the interim (between when the measurement is valid and when it is available), but that means storing the velocity at all timesteps in the interval, and recomputing the sum is unneccessary duplicated effort.

Another approach might be to keep a running average of the velocity, so that updating the second term on the right hand side of (3.39) could be done faster. This also requires keeping track of the number of timesteps since the last position measurement. This method only requires two extra pieces of data. If this running average and timestep counter were kept within the filter, the process and measurement equations would become nonlinear and unnecessarily complicated. Otherwise, an external process would still be required to deal with the running average.

The model-based approach to delayed measurement fusion takes care of all this automagically. The filter itself tracks position $\xi$ and velocity $\dot{\xi}$, effectively incrementing through (3.39) at each timestep. By keeping the a priori estimate $\hat{y}_{k+m}^{-}$as an additional state-only keeping one additional piece of information for each delayed measurement-the filter is able to compute the correct innovation and gain to fuse the information when the delayed measurement arrives. The key here is that the KF never really adjusts a posteriori estimates based on measurements-it works using innovations. 


\subsection{USBL-aided Dead Reckoning in Two Dimensions}

In this example, we study a submerged vehicle navigating by DR with position aiding from a surface tender using a USBL acoustic tracking system and an acoustic modem. To keep this example as simple as possible-and focus on the delayed measurement component of the problem-we will only consider the two-dimensional navigation problem. We make the following assumptions about measurements:

1. accurate depth (so that we may remove it from the problem),

2. accurate attitude (so that roll and pitch are compensated and we can operate in the local level frame),

3. the topside portion of the USBL measurement is treated as a black box (discussed in more detail in the next section).

\subsubsection{Mission Scenario}

The USBL system uses two-way travel time to estimate the range to a beacon, and the phase difference between signals at multiple receivers to estimate azimuth and bearing angles. This relative position measurement is combined with the GPS position of the tender. The tender transmits the resulting geodetic position fix to the submerged vehicle via acoustic modem. This is a real-world example of a delayed state problem and a good example for model-driven delayed measurement fusion.

This mission scenario is becoming more commonplace in both oceanographic and commercial sectors. The advantage of USBL is that it does not require ship time to deploy and survey transponder beacons, so it is extremely portable form one site to another. However, it does require careful calibration of sensor offsets and alignment between the transducer head, GPS, and an attitude reference. It also requires that the ship remains in the same area as the vehicle to maintain good tracking during a mission. This is a non-issue for most tethered and manned vehicles, but other options such as LBL may be more appropriate for certain types of AUV deployment. 


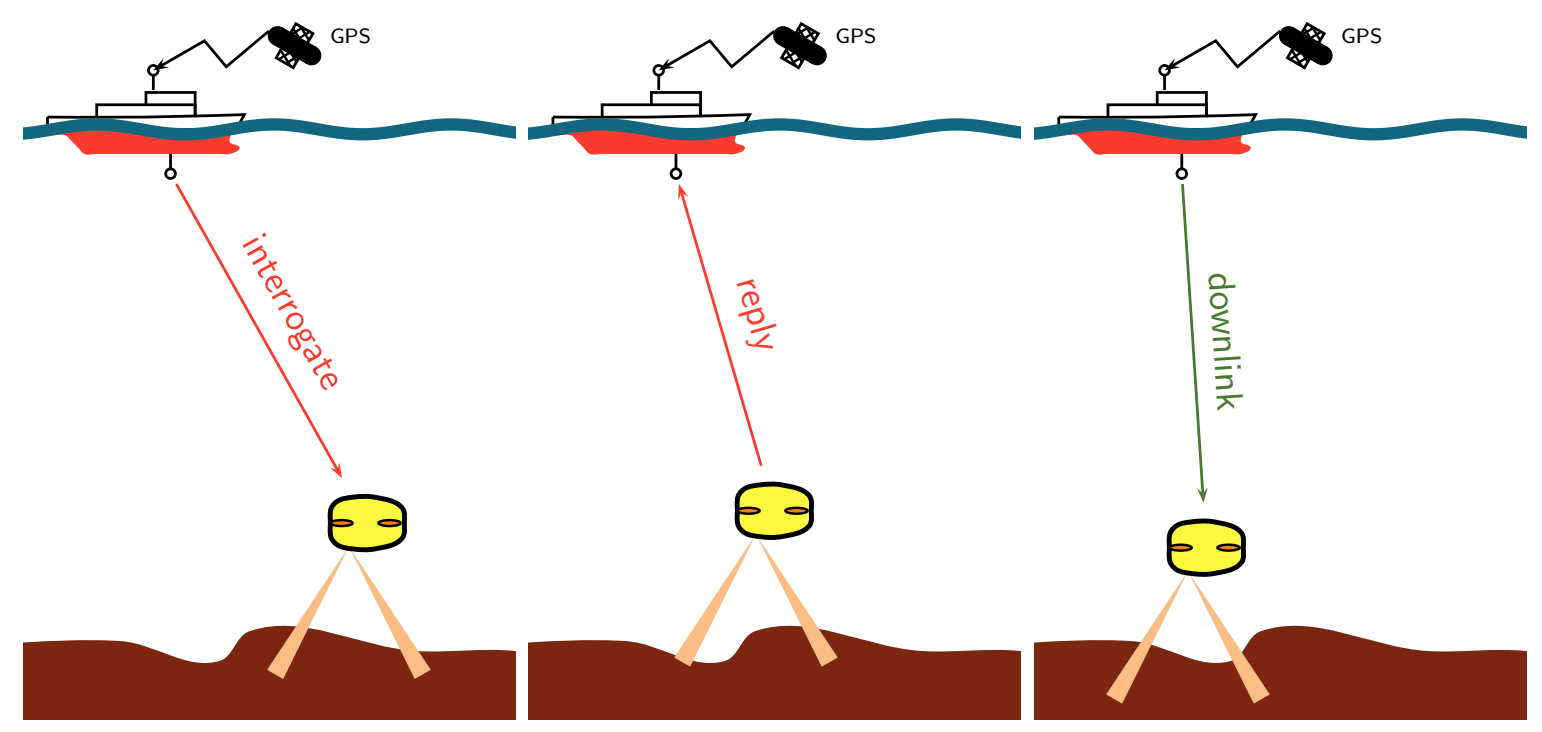

Figure 3-6: Typical measurement and communication cycle for USBL-aided DR. First (a), the surface tender interrogates the USBL beacon on the submerged vehicle. The beacon replies (b), and the USBL system reports range, bearing, and azimuth from the transducer head to the beacon. This information is fused with the GPS position and gyrocompass attitude of the tender to compute the geodetic position of the beacon. Finally (c), the position fix is transmitted to the vehicle via an acoustic modem. This cycle has a total measurement delay on the order of seconds to tens of seconds, depending on the distance between the tender and the submerged vehicle. In general, both the vehicle and the ship are moving throughout this measurement cycle.

The observation cycle in this scenario is a simple direct-path acoustic measurement. First, the surface tender interrogates a transponder on the submerged vehicle. This signal travels through the water column at the depth-averaged sound speed, $\bar{c}$, arriving at the vehicle after a one way travel time delay, $\tau_{\text {interrogate. }}$ The transponder on the vehicle then waits for a predefined turn-around-time, $\tau_{\text {tat }}$, before replying. The reply arrives back at the ship transceiver after another one way travel time delay, $\tau_{\text {reply }}$. Processing the solution for the position fix incurs another (hopefully negligi-

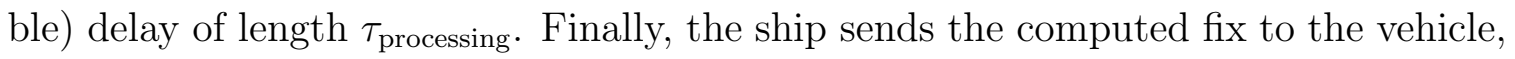
and it arrives after a third one way travel time delay, $\tau_{\text {downlink. }}$. This cycle may be interrupted for many varied reasons (e.g. downlink a command rather than a fix, lost packets, environmental or ship noise), so the vehicle should not rely on position updates for safe operation. 
USBL-aided DR actually has two delayed measurement processes. The topside process occurs on the ship, and estimates the geographic position of the transponder. The delays associated with this are $\tau_{\text {interrogate}}, \tau_{\text {tat }}$, and $\tau_{\text {reply }}$. As mentioned earlier, the topside process is treated as a black box in this example-this would be an interesting research thread to investigate in the future. The vehicle receives a fully computed geographic fix. Its measurement process is only concerned with the delays: $\tau_{\text {reply }}, \tau_{\text {processing }}$, and $\tau_{\text {downlink }}$. One convenient result of this decoupling is that the measurement delay in the process on the vehicle can be completely removed in postprocessing. We will make use of this when comparing the performance of filters with and without delay compensation.

\section{First-order, two-dimensional kinematic process model}

We want to estimate the position of a submerged vehicle by dead reckoning with position aiding from delayed USBL fixes. We will use a first-order kinematic process model, with velocity and orientation as inputs. Since depth and attitude are reliably estimated using onboard sensors, we will examine the simplified two-dimensional problem on a local North-East plane.

The position of the vehicle in the plane evolves with the first-order differential equation:

$$
\left[\begin{array}{l}
\dot{x} \\
\dot{y}
\end{array}\right]=\left[\begin{array}{cc}
\cos \psi & -\sin \psi \\
\sin \psi & \cos \psi
\end{array}\right]\left[\begin{array}{l}
u \\
v
\end{array}\right],
$$

where $x$ is positive North, $y$ is positive East, and $\psi$ is the vehicle heading. Velocities $u$ and $v$ are defined in the vehicle reference frame as forward and starboard, in accordance with [110].

Define the state vector:

$$
\boldsymbol{x}=\left[\begin{array}{l}
x \\
y
\end{array}\right]
$$


and the input vector:

$$
\boldsymbol{u}=\left[\begin{array}{l}
u \\
v \\
\psi
\end{array}\right]
$$

so that the DSSM is:

$$
\begin{aligned}
\boldsymbol{x}_{k+1} & =f\left(\boldsymbol{x}_{k}, \boldsymbol{u}_{k}, \boldsymbol{v}_{k}\right) \\
& =\boldsymbol{x}_{k}+\left(\boldsymbol{R}_{\mathbf{v}}^{\mathbf{n}}\right)_{k}\left[\begin{array}{l}
\boldsymbol{u}_{1}+\boldsymbol{v}_{1} \\
\boldsymbol{u}_{2}+\boldsymbol{v}_{2}
\end{array}\right]_{k} \Delta t
\end{aligned}
$$

where $\boldsymbol{R}_{\mathbf{v}}^{\mathbf{n}}$ is the rotation matrix from the vehicle frame to the navigation frame:

$$
\boldsymbol{R}_{\mathbf{v}}^{\mathbf{n}}=\left[\begin{array}{cc}
\cos \left(\boldsymbol{u}_{3}+\boldsymbol{v}_{3}\right) & -\sin \left(\boldsymbol{u}_{3}+\boldsymbol{v}_{3}\right) \\
\sin \left(\boldsymbol{u}_{3}+\boldsymbol{v}_{3}\right) & \cos \left(\boldsymbol{u}_{3}+\boldsymbol{v}_{3}\right)
\end{array}\right]
$$

and $\boldsymbol{v}$ is process noise.

\section{USBL measurement model}

A USBL system could report data as raw two-way travel time and phase information. In this case, the user would have to integrate platform state to compute a position fix at each beacon. Most commercial systems will output a relative or absolute fix in geodetic coordinates. This relieves the user of some responsibility, but now the system must be treated as a black box.

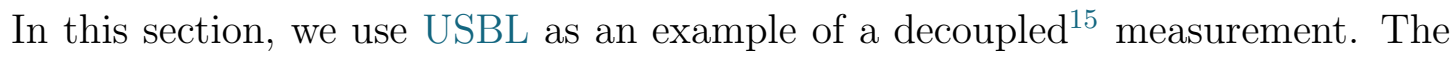
USBL sends fully-resolved position fixes to the vehicle, so it has a direct, but delayed, measurement model:

$$
\boldsymbol{y}_{k}^{\mathrm{USBL}}=h^{\mathrm{USBL}}\left(\boldsymbol{x}_{k-l}, \boldsymbol{w}_{k}\right)
$$

\footnotetext{
${ }^{15}$ This is called decoupled because each element of the measurement vector corresponds to exactly one element of the state vector.
} 


$$
=\left[x_{k-l}+\boldsymbol{w}_{1, k} \quad y_{k-l}+\boldsymbol{w}_{2, k}\right]^{T}
$$

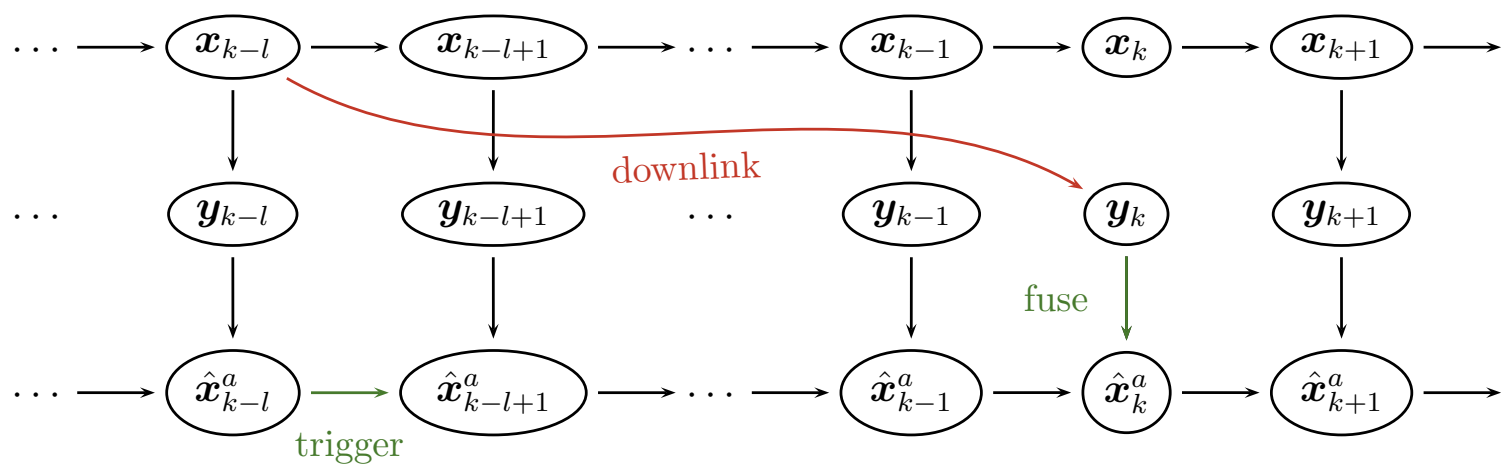

Figure 3-7: Graph of USBL-aided DR. The USBL measurement is a simple observation of the system state at an instant in the past. It draws a diagonal connection (red) from a past state to the current measurement. The trigger conditional process model and fuse conditional measurement model manage the augmented states that serve as extra memory, allowing the system to store information about the system state at the instant $k-l$, when the USBL transponder on the vehicle replied to an interrogation from the surface ship.

\subsubsection{Delay Compensation}

\section{Augmented state}

Applying the model-driven approach to delayed measurement fusion, we augment the state vector with an a priori estimate of the delayed measurement:

$$
\hat{\boldsymbol{x}}_{k}^{a-}=\left[\begin{array}{c}
\hat{\boldsymbol{x}}_{k}^{-} \\
\hat{\boldsymbol{y}}_{k+m}^{-}
\end{array}\right]=\left[\begin{array}{c}
\hat{x}_{k}^{-} \\
\hat{y}_{k}^{-} \\
\hat{x}_{k+m}^{-} \\
\hat{y}_{k+m}^{-}
\end{array}\right]
$$

\section{Conditional process models}

The augmented standard process model is now:

$$
\hat{\boldsymbol{x}}_{k+1}^{a-}=f^{\mathrm{a}}\left(\hat{\boldsymbol{x}}_{k}^{a+}, \boldsymbol{u}_{k}\right)
$$




$$
\begin{aligned}
& =\left[\begin{array}{c}
f\left(\hat{\boldsymbol{x}}_{k}^{+}, \boldsymbol{u}_{k}\right) \\
\hat{y}_{k+m}^{-}
\end{array}\right] \\
& =\hat{\boldsymbol{x}}_{k}^{a+}+\left[\begin{array}{c}
\boldsymbol{R}_{\mathrm{v}}^{\mathrm{n}}\left(\boldsymbol{u}_{3}+\boldsymbol{v}_{3}\right)_{k} \\
0 \\
0 \\
0
\end{array}\right] \Delta t
\end{aligned}
$$

and the trigger process model (to update the augmented states) is:

$$
\begin{aligned}
& \hat{\boldsymbol{x}}_{k+1}^{a-}=f^{\mathrm{u}}\left(\hat{\boldsymbol{x}}_{k}^{a+}, \boldsymbol{u}_{k}\right) \\
& =\left[\begin{array}{c}
f\left(\hat{\boldsymbol{x}}_{k}^{+}, \boldsymbol{u}_{k}\right) \\
h^{\mathrm{USBL}}\left(\hat{\boldsymbol{x}}_{k}^{+}, \boldsymbol{v}_{k}\right)
\end{array}\right] \\
& =\left[\begin{array}{cc}
\mathbf{I}_{2 \times 2} & \mathbf{0}_{2 \times 2} \\
\mathbf{I}_{2 \times 2} & \mathbf{0}_{2 \times 2}
\end{array}\right] \boldsymbol{x}_{k}^{a+}+\left[\begin{array}{c}
\boldsymbol{R}_{\mathrm{v}}^{\mathrm{n}}\left(\boldsymbol{u}_{3}+\boldsymbol{v}_{3}\right)_{k}\left[\begin{array}{c}
\boldsymbol{u}_{1}+\boldsymbol{v}_{1} \\
\boldsymbol{u}_{2}+\boldsymbol{v}_{2}
\end{array}\right]_{k} \\
0 \\
0
\end{array}\right] \Delta t
\end{aligned}
$$

The condition that chooses the correct process model will vary from problem to problem. In this example, if the submerged vehicle receives a query ping on the USBL transponder, it knows to expect a measurement in the future $\boldsymbol{y}_{k+m}$, that relates to the state now $\boldsymbol{x}_{k}$. So, a query ping serves as the trigger ${ }^{16}$ to activate conditional process model (3.50c) for one timestep.

\section{Conditional Measurement Models}

This particular (simplified) problem only has delayed measurement updates, so the only conditional measurement model is:

$$
\boldsymbol{y}_{k}=h^{\text {select }}\left(\boldsymbol{x}_{k}, \boldsymbol{w}_{k}\right),
$$

\footnotetext{
${ }^{16}$ This assumes that the USBL transponder onboard the vehicle can signal the vehicle when it has been interrogated. This may not be true for all USBL systems, but there is no fundamental reason preventing it.
} 


$$
\begin{aligned}
\hat{\boldsymbol{y}_{k}^{-}} & =\left[\begin{array}{ll}
\mathbf{0}_{2 \times 2} & \mathbf{I}_{2 \times 2}
\end{array}\right] \hat{\boldsymbol{x}}_{k}^{a}, \\
& =\hat{\boldsymbol{y}}_{k+m}^{-} .
\end{aligned}
$$

Recall that the dummy index $m$ is zero when the delay is over and the measurement is fused.

Now that the augmented state vector is defined, along with appropriate conditional process and measurement models, this delayed-state sigma point Kalman filter (DS-SPKF) can be applied to filter real data. We will use field data from a recent deployment of the AUV Sentry in this example.

\subsubsection{Sentry059: Galápagos Ridge}

Dive 059 was a standard bathymetric mapping survey along a portion of the Galápagos rift. Figure 3-8 shows a map view of the first survey block, which provides the navigation dataset for this example. The dive starts in the Northwest corner with a short lead-in to the first survey block. Tracklines are oriented East-West, progressing Southward in a classical lawnmower pattern. The block ends with a Northward crossing line to provide data for later adjustments based on the multibeam data.

The goal here is to characterize the relative performance of two UKFs: one ignoring measurement latency, and the other using augmented states and conditional models to treat the delayed measurements properly. Since this dataset consists of real DR and USBL field measurements, there is no absolute ground truth to use as a baseline. Instead, we adopt a three filter approach: one idealized filter serves as the basis from which to compare the other two. All filters are UKFs operating with the same process noise and measurement noise (Table 3.3).

The first filter is the baseline, it operates in postprocessing with complete knowledge of the measurement delays. This filter receives measurements at the instant they are valid, and fuses them immediately. We will call it the zero delay filter because it is analogous to the zero delay case in the earlier DHO example.

The second filter is a traditional UKF based on (3.44) and (3.47). It receives 


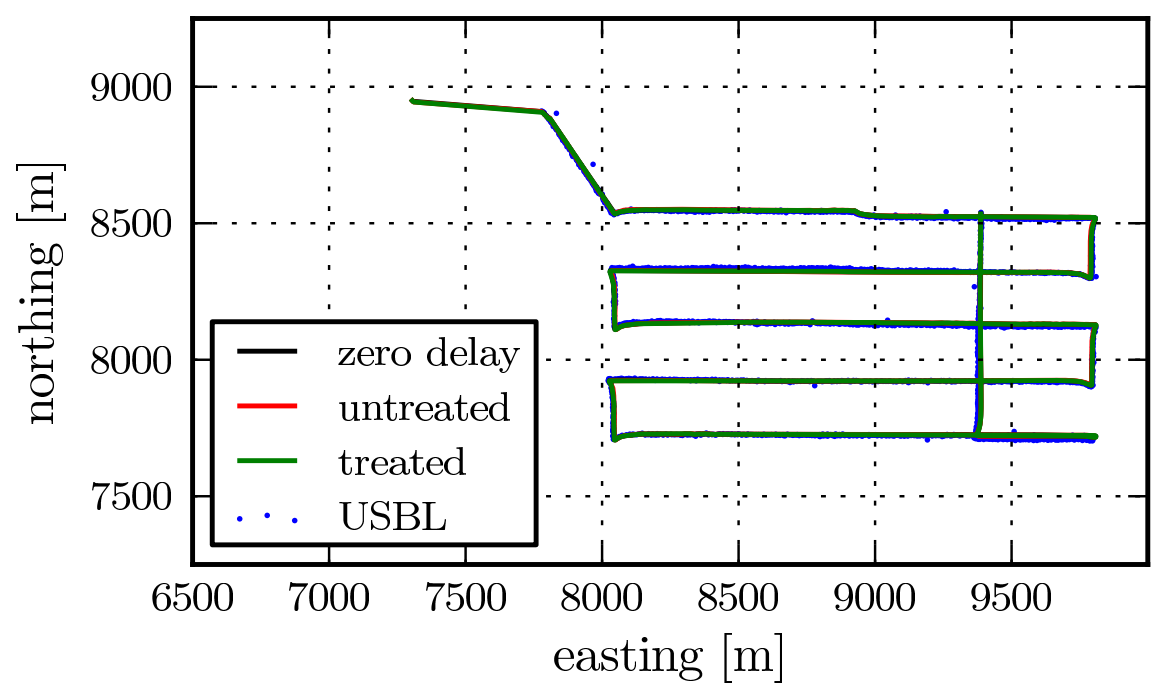

Figure 3-8: Map view of the first survey block on Sentry dive 059. The tracklines in this block are oriented East-West, which is convenient for comparing filters with and without delay compensation since delays manifest as alongtrack error. USBL fixes are shown as blue dots. The black, red, and green lines are position estimates given by the UKF with no delay, untreated delay, and treated delay, respectively. The estimates are indistinguishable at this scale-Figure 3-9 shows the differences between them. 
Table 3.3: Timing, process noise, and measurement noise parameters used in renavigation of dive 059 .

\begin{tabular}{c|c|c|c|c|c}
\hline$T^{\text {fast }}$ & $T^{\text {slow }}$ & $l$ & $\sigma_{\boldsymbol{v}_{1}}, \sigma_{\boldsymbol{v}_{2}}$ & $\sigma_{\boldsymbol{v}_{3}}$ & $\sigma_{\boldsymbol{w}_{1}}, \sigma_{\boldsymbol{w}_{2}}$ \\
$\mathrm{~s}$ & $\mathrm{~s}$ & - & $\mathrm{m} / \mathrm{s}$ & $\mathrm{rad}$ & $\mathrm{m}$ \\
\hline \hline 0.2 & $\mathcal{O}(1-10)$ & $20-30$ & 0.04 & 0.001 & 5.0 \\
\hline
\end{tabular}

measurements after the USBL has computed a fix and it has been sent to the vehicle via acoustic modem ${ }^{17}$. However, this filter ignores the fact that these measurements are delayed, so we call its estimates 'untreated.'

The third filter uses the delayed-state Kalman filter (DS-KF) approach. It receives identical measurements at the same time as the second filter, but calculates the correct delayed residual before fusing them with the current state. We call the estimates from this filter 'treated.'

We compare the relative performance of filters 2 and 3 by examining the position residuals of the treated and untreated estimates. These residuals are defined:

$$
\begin{gathered}
\boldsymbol{r}^{\text {untreated }}=\boldsymbol{x}^{\text {zero delay }}-\boldsymbol{x}^{\text {untreated }} \\
\boldsymbol{r}^{\text {treated }}=\boldsymbol{x}^{\text {zero delay }}-\boldsymbol{x}^{\text {treated }}
\end{gathered}
$$

Recall that these are not residuals relative to a ground-truth.

Figure 3-9 shows absolute values for North and East residuals, as well as the magnitude of the total two-dimensional residual vector. The untreated estimates are worse alongtrack (i.e. East-West) than across-track (i.e North-South). This is expected because the delayed measurements correspond to spatial errors opposite the direction of travel. Note that the treated estimates are much closer to the baseline by all measures. Furthermore, the residuals of the treated measurements are lower than the USBL measurement noise standard deviation.

\footnotetext{
${ }^{17}$ Automatic acoustic downlink of position fixes was not implemented for dive 059, so this timing has been estimated based on two-way travel times measured during the dive.
} 

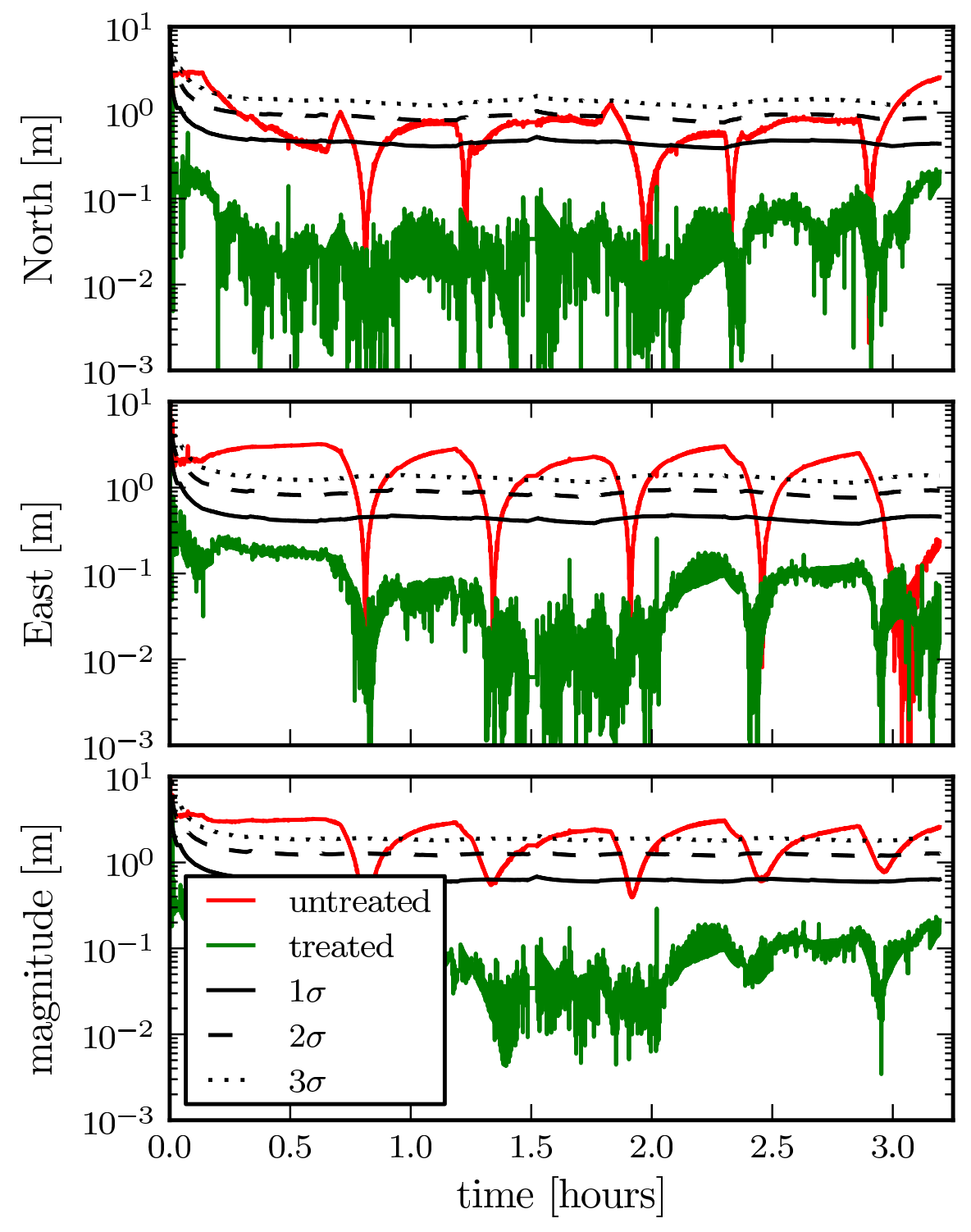

Figure 3-9: Absolute values of position residuals from the UKF estimates with untreated delay (red) and treated delay (green), plotted on a log scale. The baseline is given by position estimates of the UKF postprocessed with zero delay. $1 \sigma, 2 \sigma$, and $3 \sigma$ confidence intervals of the zero delay filter are shown in black for reference. 


\subsection{LBL-aided Dead Reckoning in Three Dimensions}

This section demonstrates the model-driven approach to delayed measurement fusion on another application in underwater navigation-LBL-aided dead reckoning. We will briefly review long baseline acoustic navigation, then define the standard kinematic process model, then define the LBL measurement model. We then define an augmented state and adjusted process and measurement models to support asynchronous, delay-compensated aiding from the LBL system. We demonstrate the delayed measurement SPKF using navigational data from Sentry dive 073-a preliminary bathymetric mapping mission within a three-transponder LBL net.

\subsubsection{Mission Scenario}

Long baseline acoustic positioning (LBL) provides delayed, but drift free, measurements of vehicle position at a low update rate. In precision underwater navigation, LBL measurements bound the error growth of high-rate DR or INS navigation.

LBL positioning uses ranges from two or more fixed underwater acoustic transponders to localize another transponder, usually on a submerged vehicle or instrument package [3]. The globally referenced transponder positions are surveyed by a ship when the LBL net is deployed. In spherical LBL positioning ${ }^{18}$, the vehicle interrogates the net with an acoustic signal, and each transponder replies in a unique way. Given an estimate of the sound speed through the water, the measured two-way travel times produce estimates of the slant range between the vehicle and each transponder in the net.

LBL works like an acoustic version of GPS underwater-you just have to provide your own beacons (satellites). The cost is in the ship time spent deploying and surveying the transponders ${ }^{19}$-once that is done, the ship is free to leave the area for

\footnotetext{
${ }^{18}$ Hyperbolic LBL positioning, on the other hand, uses a passive receiver and synchronized beacons. The receiver position is calculated using the difference in ranges to the two beacons $[4,5]$. We will focus on spherical LBL for the remainder of this section.

${ }^{19}$ and recovering them afterward
} 
other tasks. An AUV can continue to operate unattended without position updates from the ship and without its navigation error growing unbounded. This flexibility makes LBL a good solution when operations will be in the same area for several days.

However, the travel times and subsequent slant ranges used in LBL positioning are inherently delayed. This motivates an explicit and rigorous treatment of the delay, and makes LBL-aided DR an interesting example application for the model-driven approach to delayed measurement fusion presented in section 3.3.

\section{Second-order, three dimensional kinematic process model}

Using the standard notation for a submerged body $[110,111]$, the state is:

$$
\begin{aligned}
\boldsymbol{x} & =\left[\begin{array}{lll}
\boldsymbol{\eta}_{1}^{T} & \boldsymbol{\nu}_{1}^{T} & \boldsymbol{\eta}_{2}^{T}
\end{array}\right]^{T} \\
& =\left[\begin{array}{lllllllll}
x & y & z & u & v & w & \phi & \theta & \psi
\end{array}\right]^{T}
\end{aligned}
$$

The positions $x, y, z$ are positive North, East, and Down in the local navigation frame. The velocities $u, v, w$ are positive forward, starboard, and toward the keel in the moving vehicle frame. And, the Euler angles ${ }^{20} \phi, \theta, \psi$ describe roll, pitch, and heading.

The process model is:

$$
\begin{aligned}
\hat{\boldsymbol{x}}_{k+1}^{-} & =f\left(\hat{\boldsymbol{x}}_{k}^{+}, \boldsymbol{u}_{k}, \boldsymbol{v}_{k}\right) \\
& =\hat{\boldsymbol{x}}_{k}^{+}+\left[\begin{array}{c}
\left(\boldsymbol{R}_{\mathbf{v}}^{\mathbf{n}}\right)_{k}\left(\hat{\boldsymbol{x}}_{3: 6}\right)_{k}^{+} \\
\boldsymbol{u}_{k}+\boldsymbol{v}_{k}
\end{array}\right] \Delta t
\end{aligned}
$$

where $\left(\boldsymbol{R}_{\mathbf{v}}^{\mathbf{n}}\right)_{k} \in S O(3)$ is the rotation matrix from the vehicle frame to the navigation frame, calculated from the Euler angles.

\footnotetext{
${ }^{20}$ Alternate attitude representations are better, but Euler angles are used in this example because 1) this is standard, so it will be most accessible 2) the fiber-optic gyroscope (FOG) on Sentry ouputs Euler angles in its standard configuration 3) the FOG measurement is precise enough that it is effectively driving the attitude variables as a control input.
} 


\section{DVL measurement model}

The measurement equation for DVL bottom-track velocities in instrument coordinates is:

$$
\begin{aligned}
\boldsymbol{y}_{k}^{\mathrm{DVL}}=h^{\mathrm{DVL}, \mathrm{i}}\left(\boldsymbol{x}_{k}, \boldsymbol{w}_{k}\right) \\
=\mathbf{R}_{\mathrm{v}}^{\mathrm{i}}\left[\begin{array}{c}
u_{k} \\
v_{k} \\
w_{k}
\end{array}\right]+\boldsymbol{w}_{k} \\
\hat{\boldsymbol{y}}_{k}^{-}=\mathbf{R}_{\mathrm{v}}^{\mathrm{i}}\left[\begin{array}{c}
\hat{u}_{k}^{-} \\
\hat{v}_{k}^{-} \\
\hat{w}_{k}^{-}
\end{array}\right]
\end{aligned}
$$

where $\mathbf{R}_{\mathrm{v}}^{\mathrm{i}}$ is the rotation matrix describing the misalignment between vehicle and DVL instrument reference frames.

\section{FOG measurement model}

The measurement equation for vehicle orientation from the FOG is:

$$
\begin{aligned}
\boldsymbol{y}_{k}^{\mathrm{FOG}}= & h^{\mathrm{FOG}}\left(\boldsymbol{x}_{k}, \boldsymbol{w}_{k}\right) \\
= & {\left[\begin{array}{c}
\phi_{k} \\
\theta_{k} \\
\psi_{k}
\end{array}\right]+\boldsymbol{w}_{k} } \\
\hat{\boldsymbol{y}}_{k}^{-}= & {\left[\begin{array}{c}
\hat{\phi}_{k}^{-} \\
\hat{\theta}_{k}^{-} \\
\hat{\psi}_{k}^{-}
\end{array}\right] }
\end{aligned}
$$

\section{Standard LBL measurement model}

Traditional LBL without delay compensation Figure 3-10 assumes that the vehicle and beacon are stationary over the interrogation period and that returns from all 


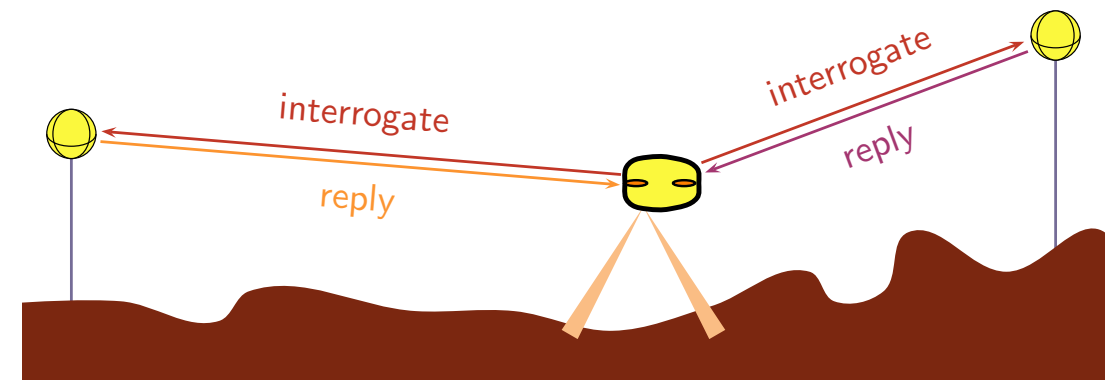

Figure 3-10: LBL acoustic positioning using two-way travel times from multiple underwater transponders. The vehicle interrogates the net, and each transponder replies on its own unique frequency. The two-way travel times give estimated ranges to each transponder. Combined with the known positions of the transponders, the ranges provide an observation of the vehicle position.

beacons are available at the same time. The position of the vehicle is then calculated by trilateration. If more ranges are available, the problem is solved using nonlinear least squares (LS) [112]:

$$
\hat{\boldsymbol{x}}_{k}=\underset{\boldsymbol{x}_{k}}{\operatorname{argmin}} \sum_{i=0}^{n}\left\|\left(\frac{\bar{c}}{\tau_{\mathrm{twtt}}}\right)_{i}-\right\| \boldsymbol{x}_{b_{i}}-\boldsymbol{x}_{k}\|\|
$$

We could use LBL measurements like this in the same loosely-coupled fashion that we used USBL measurements in section 3.5. However, the complex nature of the delay in LBL measurements suggests a different way.

\section{Asynchronous LBL measurement model}

Here we use LBL-aiding as an example of a more tightly integrated measurement. We update the position estimate of the vehicle asynchronously, fusing travel time innovations as the response from each beacon in the net is received. One advantage of this approach is that the navigator can perform a partial position update even if 
it only gets a return from one transponder. In section 3.6 .2 we will adapt this to explicitly account for the measurement latency.

The measurement is the two-way travel time:

$$
\begin{aligned}
\boldsymbol{y}_{k}^{\mathrm{LBL}} & =h^{\mathrm{LBL}}\left(\boldsymbol{x}_{k}, \boldsymbol{w}_{k}\right) \\
& =2 \bar{c}\left(\boldsymbol{r}_{\mathrm{b}}^{\mathrm{v}}\right)_{k}+\tau_{\text {turn }}+\boldsymbol{w}_{k},
\end{aligned}
$$

so the a priori estimate is:

$$
\begin{aligned}
\hat{\boldsymbol{y}}_{k}^{-} & =h^{\mathrm{LBL}}\left(\hat{\boldsymbol{x}}_{k}^{-}\right) \\
& =2 \bar{c}\left(\hat{\boldsymbol{r}}_{\mathrm{b}}^{\mathrm{v}}\right)_{k}+\tau_{\text {turn }},
\end{aligned}
$$

where $\bar{c}$ is the average soundspeed ${ }^{21}, \tau_{\text {turn }}$ is the turn-around-time for the beacon, and the range from the vehicle to the beacon is geometrically defined:

$$
\begin{aligned}
& \left(\boldsymbol{r}_{\mathrm{v}}^{\mathrm{b}}\right)_{k}=\left(\boldsymbol{r}_{\mathrm{b}}^{\mathrm{v}}\right)_{k}=\sqrt{\left(\boldsymbol{x}_{b_{i}}-\boldsymbol{x}_{k}\right)\left(\boldsymbol{x}^{\mathrm{b}}-\boldsymbol{x}^{\mathrm{v}}\right)^{T}} \\
& \left(\hat{\boldsymbol{r}}_{\mathrm{v}}^{\mathrm{b}}\right)_{k}=\left(\hat{\boldsymbol{r}}_{\mathrm{b}}^{\mathrm{v}}\right)_{k}=\sqrt{\left(\boldsymbol{x}_{b_{i}}-\hat{\boldsymbol{x}}_{k}^{-}\right)\left(\boldsymbol{x}^{\mathrm{b}}-\hat{\boldsymbol{x}}_{k}^{-}\right)^{T}} .
\end{aligned}
$$

\section{Realtime multipath and outlier rejection}

The noise in LBL travel times is non-gaussian and multimodal [112,114], outliers cannot simply be rejected using the confidence bounds of the KF. Here we use the field-proven three-test architecture from [112]. For a travel time to be considered valid, it must pass three tests: a median test, a bounce test, and a wrap test. The last two are a specific implementation of range gating for multipath, while the median test removes obvious outliers. This is an online method, making it a good match to run within the LBL measurement model of the asynchronous filter presented here.

\footnotetext{
${ }^{21}$ Depending on signal path and sound velocity profile, this could be a complicated function with many different possible values for the interrogation and response. Here we assume direct-path for simplicity. Multipath and parallel hypothesis [113] LBL measurement models are a topic of future research.
} 


\subsubsection{Delay Compensation}

Careful thought about the nature of a two-way travel time LBL measurement leads to the realization that a two-way travel time is really the sum of two one-way travel times. This may seem obvious, but consider: it is actually the sum of two different one-way travel times ${ }^{22}$ (see Figure 3-11):

interrogation the travel time from the vehicle to the beacon, and

reply the travel time from the beacon back to the vehicle.

Assuming the beacon is stationary (as we have been all along), each of these travel times can be estimated independently by the vehicle in realtime. The interrogation travel time is based on the range from the vehicle to the beacon at the instant the vehicle pings the net:

$$
\begin{aligned}
\boldsymbol{y}_{k-l}^{\text {owtt }}=\tau_{\text {interrogate }}^{b_{i}} & =h^{\text {owtt }}\left(\boldsymbol{x}_{k-l}, \boldsymbol{x}_{b_{i}}, c(z)\right), \\
& =\frac{1}{\bar{c}}\left\|\boldsymbol{r}_{k-l}^{b_{i}}\right\|_{2}, \\
& =\frac{1}{\bar{c}} \sqrt{\left(\boldsymbol{x}_{k}-\boldsymbol{x}_{b_{i}}\right)\left(\boldsymbol{x}_{k}-\boldsymbol{x}_{b_{i}}\right)^{T}} .
\end{aligned}
$$

The reply travel time is based on the range from the beacon to the vehicle at the instant the vehicle receives the return:

$$
\begin{aligned}
\boldsymbol{y}_{k}^{\text {owtt }}=\tau_{\text {reply }}^{b_{i}} & =h^{\text {owtt }}\left(\boldsymbol{x}_{k}, \boldsymbol{x}_{b_{i}}, c(z)\right), \\
& =\frac{1}{\bar{c}}\left\|\boldsymbol{r}_{k}^{b_{i}}\right\|_{2}, \\
& =\frac{1}{\bar{c}} \sqrt{\left(\boldsymbol{x}_{k}-\boldsymbol{x}_{b_{i}}\right)\left(\boldsymbol{x}_{k}-\boldsymbol{x}_{b_{i}}\right)^{T}} .
\end{aligned}
$$

\footnotetext{
${ }^{22}$ This is not a novel observation, but many existing implementations ignore this fact. Explicit delay compensation is necessary to handle an LBL measurement this way. After formulating the problem independently using the model-driven approach presented in this research, it was brought to my attention that a similar treatment existed in [104]. That work uses state augmentation with a full state copy to handle delayed measurements, as discussed in section 3.1.2.
} 


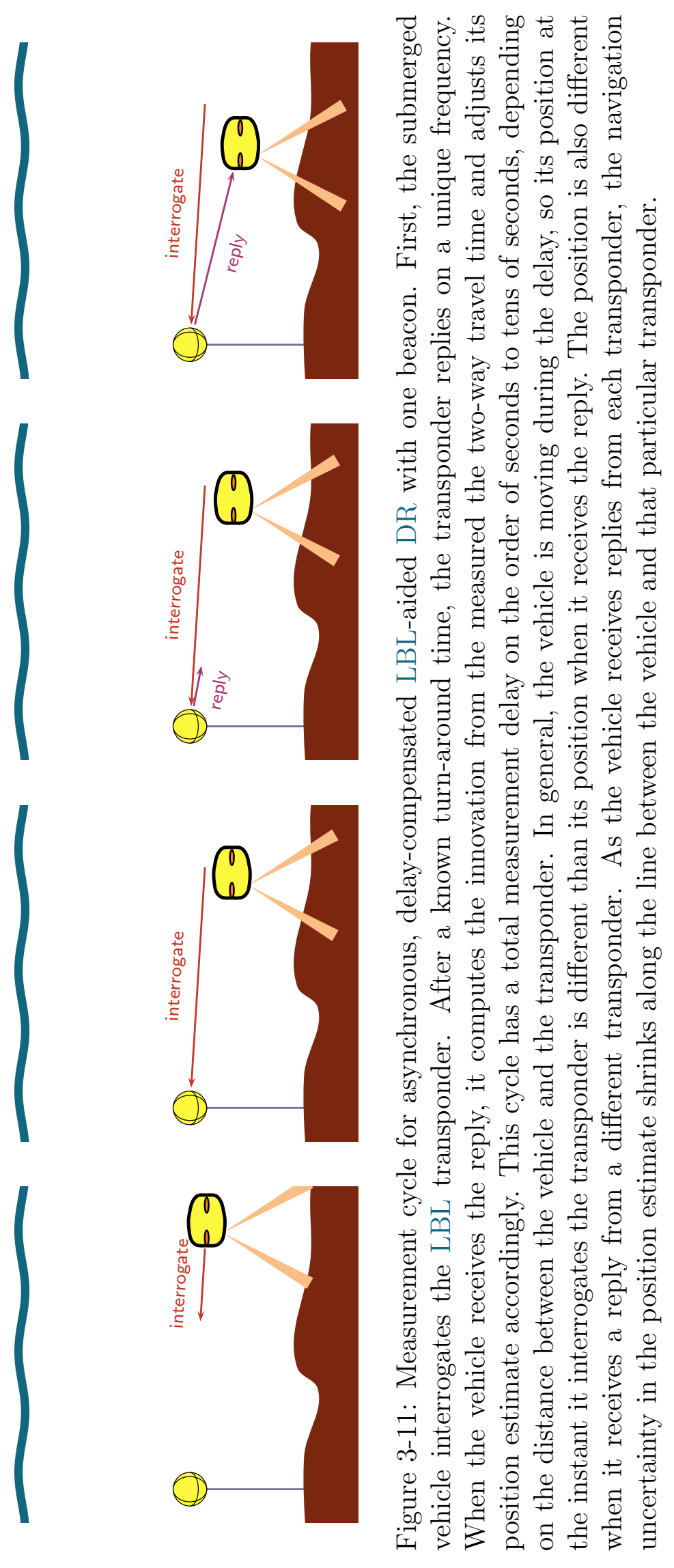


These combine in the LBL measurement of the true two-way travel time:

$$
\begin{aligned}
\boldsymbol{y}_{k}^{\mathrm{twtt}} & =h^{\mathrm{twtt}}\left(\boldsymbol{x}_{k}, \boldsymbol{x}_{k-l}, \mathbf{x}_{b_{i}}, c(z)\right), \\
& =\tau_{\text {interrogate }}^{b_{i}}+\tau_{\text {tat }}+\tau_{\text {reply }}^{b_{i}}, \\
& =h^{\text {owtt }}\left(\boldsymbol{x}_{k-l}, \mathbf{x}_{b_{i}}, c(z)\right)+t_{\text {tat }}+h^{\text {owtt }}\left(\boldsymbol{x}_{k}, \mathbf{x}_{b_{i}}, c(z)\right), \\
& =\tau_{\text {tat }}+\frac{1}{\bar{c}}\left(\sqrt{\left(\boldsymbol{x}_{k}-\boldsymbol{x}_{b_{i}}\right)\left(\boldsymbol{x}_{k}-\boldsymbol{x}_{b_{i}}\right)^{T}}+\sqrt{\left(\boldsymbol{x}_{k}-\boldsymbol{x}_{b_{i}}\right)\left(\boldsymbol{x}_{k}-\boldsymbol{x}_{b_{i}}\right)^{T}}\right),
\end{aligned}
$$

which replaces the earlier form (3.61b) that did not capture the delay.

With a more complete understanding of the two-way travel time measurement, we are now ready to apply the model-driven approach to delayed measurement fusion.

\section{Augmented state}

The LBL measurement equation (3.66) is an example of a composite measurement delay-the actual measurement is a combination of delayed and undelayed parts (Figure 3-12). Instead of using an a priori estimate of the whole two-way travel time as the augmented state, we will only use the part we can calculate at the trigger instant $(k-l)$. This is the interrogation travel time, or the one-way travel time from the vehicle to the beacon. We will have one augmented state for each beacon in the net ${ }^{23}$ :

$$
\hat{\boldsymbol{x}}_{k}^{a}=\left[\begin{array}{c}
\hat{\boldsymbol{x}}_{k} \\
\tau_{\text {interrogate }}^{b_{1}} \\
\tau_{\text {interrogate }}^{b_{2}} \\
\vdots \\
\tau_{\text {interrogate }}^{b_{j}}
\end{array}\right]=\left[\begin{array}{c}
\hat{\boldsymbol{x}}_{k} \\
\hat{\boldsymbol{y}}_{k+m}^{\text {owtt }, 1-} \\
\hat{\boldsymbol{y}}_{k+m}^{\text {owtt, } 2-} \\
\cdots \\
\hat{\boldsymbol{y}}_{k+m}^{\text {owtt }, j-}
\end{array}\right]
$$

\footnotetext{
${ }^{23}$ In applications with four or more beacons, using the vehicle position for the augmented states would lower the dimension of the filter slightly. The measurement model would then need to compute a priori estimates of one-way travel times for both interrogation and reply before calculating the two-way travel time and the measurement innovation. The difference in dimensionality is so small that this becomes a matter of personal preference.
} 


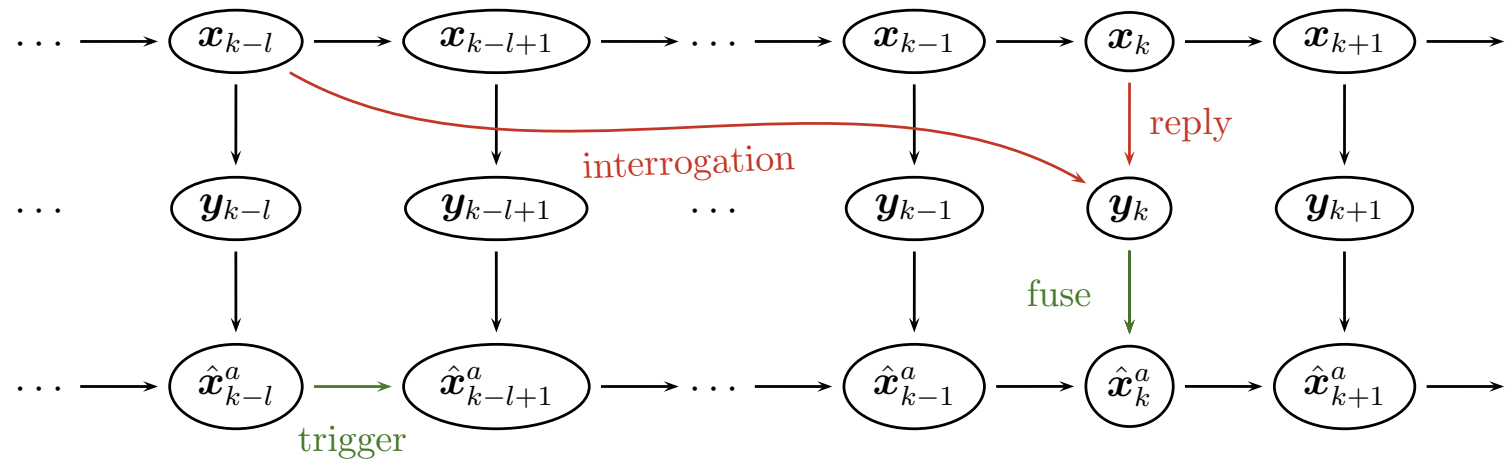

Figure 3-12: Graph of asynchronous LBL-aided DR. The LBL measurement is a composite observation of the system state at two distinct instants, and involves both diagonal and vertical connections (red). The trigger conditional process model and fuse conditional measurement model manage the augmented states that serve as extra memory, allowing the system to store information about the system state at the interrogation instant $k-l$.

\section{LBL interrogation process model}

We update the augmented state for each of the beacons individually at the interrogation instant. Keeping the convention of looking back in time on the trigger, the interrogation instant is $(k-l)$, and the conditional process model at that instant is:

$$
\hat{\boldsymbol{x}}_{k-l}^{a-}=\left[\begin{array}{c}
\hat{\boldsymbol{x}}_{k-l-1}^{a+} \\
h^{\text {owtt }}\left(\hat{\boldsymbol{x}}_{k-l-1}, \boldsymbol{x}_{b_{1}}, c(z)\right) \\
h^{\text {owtt }}\left(\hat{\boldsymbol{x}}_{k-l-1}, \boldsymbol{x}_{b_{2}}, c(z)\right) \\
\vdots \\
h^{\text {owtt }}\left(\hat{\boldsymbol{x}}_{k-l-1}, \boldsymbol{x}_{b_{j}}, c(z)\right)
\end{array}\right]
$$

The default process model simply leaves the augmented states unchanged, as usual.

\section{Delay-compensated, asynchronous LBL measurement model}

Just as with the uncompensated asynchronous LBL model, we fuse the information from each two-way travel time as it becomes available. The difference is that we use the augmented states and (3.66) to compute the a priori estimate of the full measurement, and compute the travel time innovation based on that. The measurement 
equation is:

$$
\begin{aligned}
\hat{\boldsymbol{y}}_{k}^{-} & =h^{a-\text { twtt }}\left(\hat{\boldsymbol{x}}_{k}^{a}, \boldsymbol{x}_{b_{i}}, c(z)\right) \\
& =\tau_{\text {interrogate }}^{b_{i}}+\tau_{\text {tat }}+\tau_{\text {reply }}^{b_{i}}, \\
& =h^{\text {select }}\left(\hat{\boldsymbol{x}}_{k}^{a}, b_{i}\right)+\tau_{\text {tat }}+h^{\text {owtt }}\left(\hat{\boldsymbol{x}}_{k}^{a}, \mathbf{x}_{b_{i}}, c(z)\right),
\end{aligned}
$$

where $h^{\text {select }}\left(\hat{\boldsymbol{x}}_{k}^{a}, b_{i}\right)$ simply selects the interrogation one-way travel time for the relevant transponder from the augmented states.

The filter continues to run until it receives a reply from another beacon, at which time it fuses that measurement. The augmented states are not updated until the interrogation at the beginning of the next LBL cycle.

\subsubsection{Sentry073: Håkon Mosby Mud Volcano}

This section evaluates the performance of the asynchronous LBL-aided DR filter using navigational data from field deployments of the oceanographic AUV Sentry. We will compare the estimates from the fully delay compensated filter to two different filters:

interrogate assumes the two-way travel time is an observation of the system state only at the time the vehicle interrogates the net ${ }^{24}$, and

reply assumes the two-way travel time is an observation of the system state only at the time the vehicle receives the reply.

Unfortunately, due to the complex nature of LBL measurement delay, it is not possible to implement a comparable filter with the delays artificially removed ${ }^{25}$ as we did in section 3.5.3.

The LBL-aided DR filter was used to renavigate data from Sentry073, the initial multibeam survey during a research cruise in September/October 2010 on the Håkon

\footnotetext{
${ }^{24}$ Note that this filter still requires delay treatment, because the two-way travel time measurement is not available at the interrogation instant.

${ }^{25}$ One might consider doing this in a more traditional LBL system with explicit calculation of geographic position fixes, but that is not directly comparable to the asynchronous approach taken here.
} 


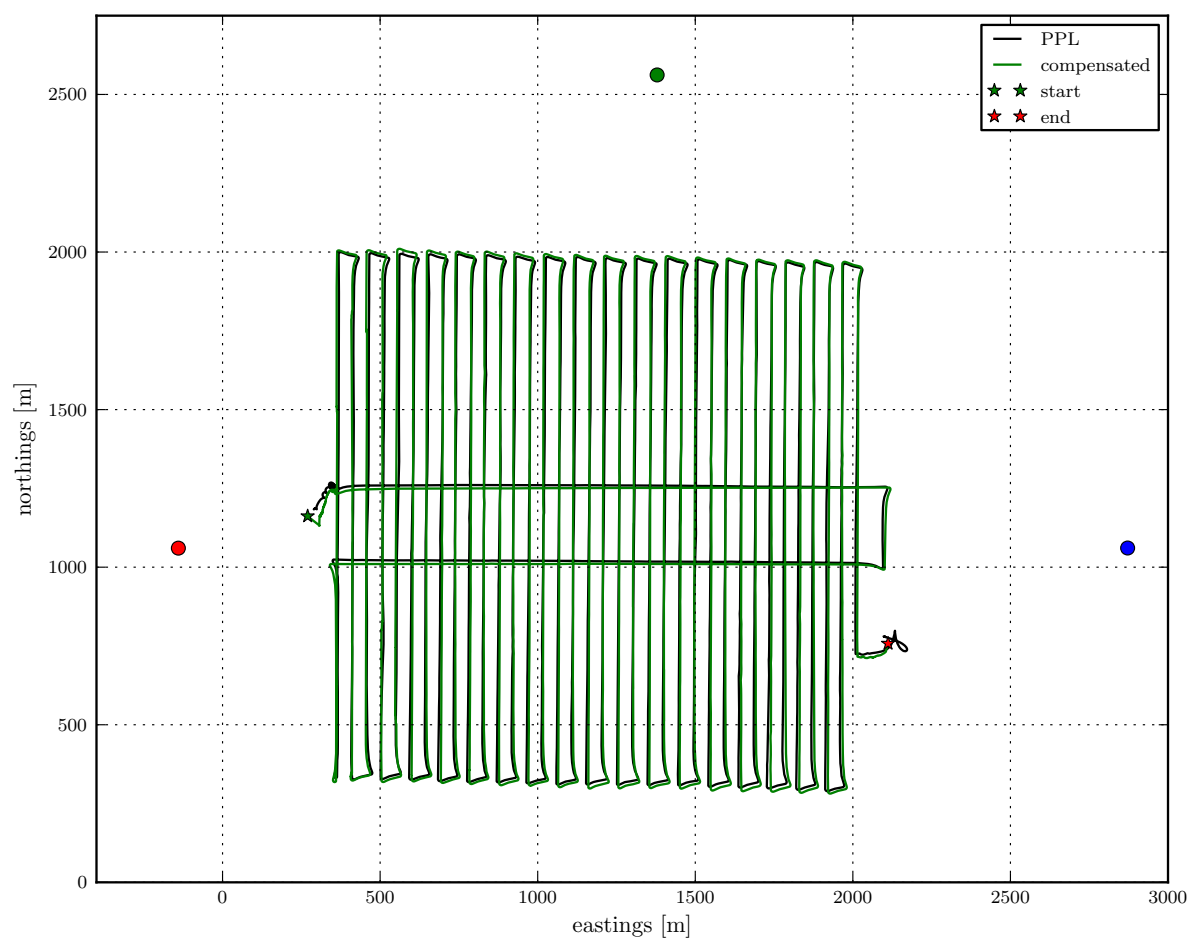

Figure 3-13: Map view of the initial multibeam survey mission Sentry dive 073. The tracklines in this block are oriented North-South. The locations of the three transponders in the $12 \mathrm{kHz}$ LBL net are marked by colored circles. The black line marked PPL uses the DSL standard postprocessing with traditional LBL and explicit position fixes. The green line is the fully delay compensated realtime LBL-aided DR filter detailed in this section. The fundamental difference in LBL measurement fusion makes a direct comparison of these two trajectories questionable, but the PPL track is useful as a sanity check for the realtime filter.

Mosby mud volcano in the Barents Sea. These dives were part of a research cruise led by Dr. Antje Boetius with the goal of characterizing the bathymetry, sub-bottom composition, and chemistry surrounding the mud volcano.

Figure 3-13 shows the trajectory of Sentry during this first dive, and the locations of the three transponders in the LBL net that the Sentry team deployed and surveyed prior to the dive. After two crossing lines at the beginning of the dive, the tracklines are primarily North-South, covering over 68 kilometers at an average speed of $1 \mathrm{~m} / \mathrm{s}$ and and average depth of $1245 \mathrm{~m}$. Figure 3-14 shows the components of the vehicle position throughout the dive. 


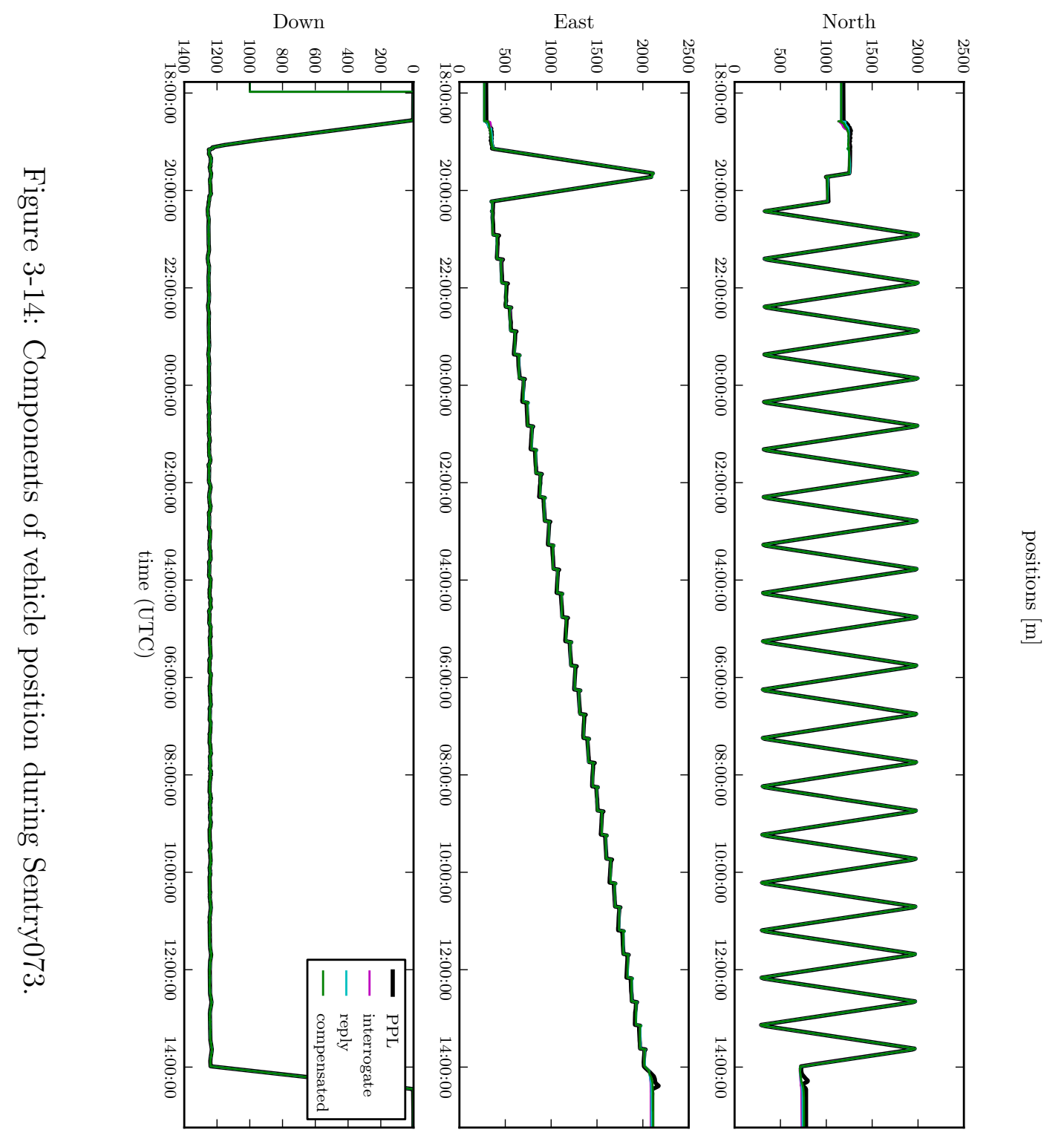



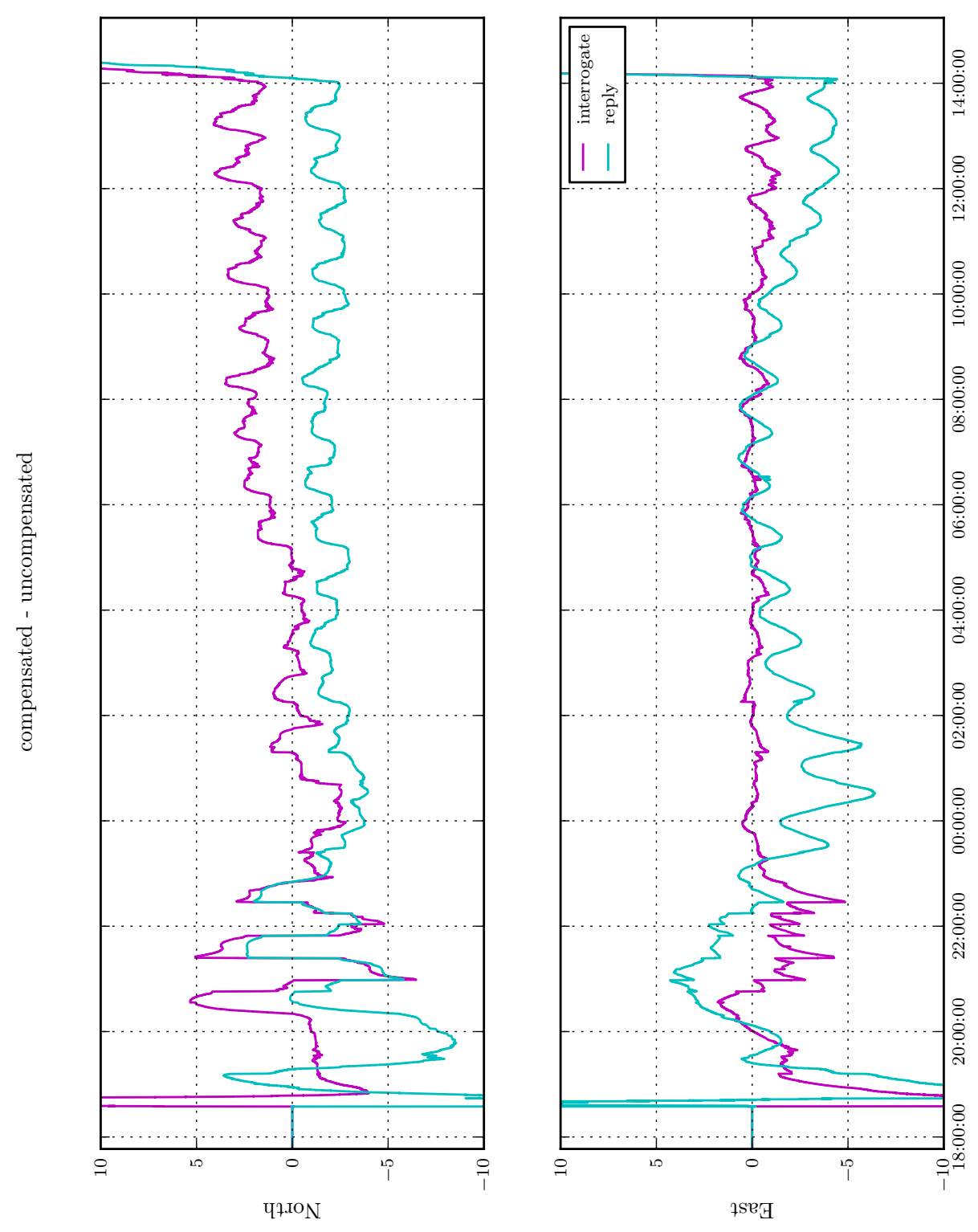

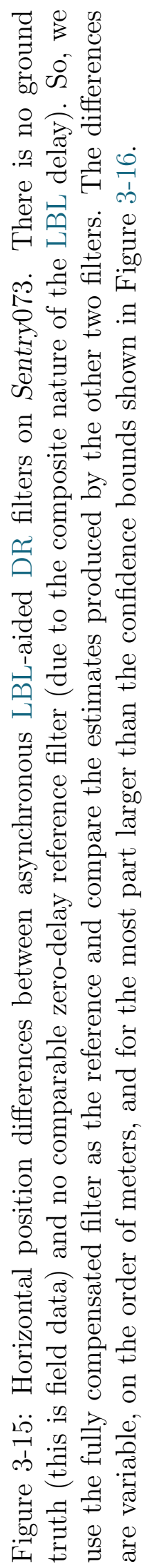


Figure 3-14 shows the horizontal ${ }^{26}$ components of the difference between vehicle position estimated by the fully delay compensated filter and the filters assuming $\tau^{\text {twtt }}$ is based solely on the interrogation state and assuming $\tau^{\text {twtt }}$ is based solely on the reply state, respectively. The position estimates of the different filters vary on the order of 1-10 m, with a periodicity that may be linked to the change in directions progressing through tracklines. Unfortunately, without an independent ground truth, it is difficult to say with certainty which estimate is most correct-the measurement equation (3.66) for the fully delay compensated filter certainly reflects the true nature of the two-way travel time most closely. Figure 3-16 shows the filter confidence intervals in North and east directions throughout the course of the dive. Despite having the same filter parameters and receiving the exact same measurements from all navigational sensors, the filters with LBL measurement models based solely on interrogation state and reply state have larger confidence intervals than the fully delay compensated filter. This indicates that the models in the fully delay compensated filter may be more consistent with the measurements it is receiving.

\subsection{Discussion and Conclusions}

We've presented a flexible, model-driven approach to fuse information from delayed measurements in realtime. We used the proven framework of Kalman filter, and our model-driven approach does not change any of the underlying machinery of the KF. The delay treatment is mathematically rigorous and optimal for linear systems-it is a tractable, efficient, and principled approach for nonlinear systems. We characterized the estimation error using a simulation of a simple canonical system, then applied the approach to the real-world problem of navigating an underwater vehicle in the deep ocean, both with a decoupled example (USBL-aided DR) and with a more integrated example (LBL-aided DR).

This is really just the beginning. With a straightforward approach to delayed

\footnotetext{
${ }^{26}$ We don't show the vertical component simply because undelayed measurement from the pressure depth sensor constrain this state similarly for all three filters.
} 

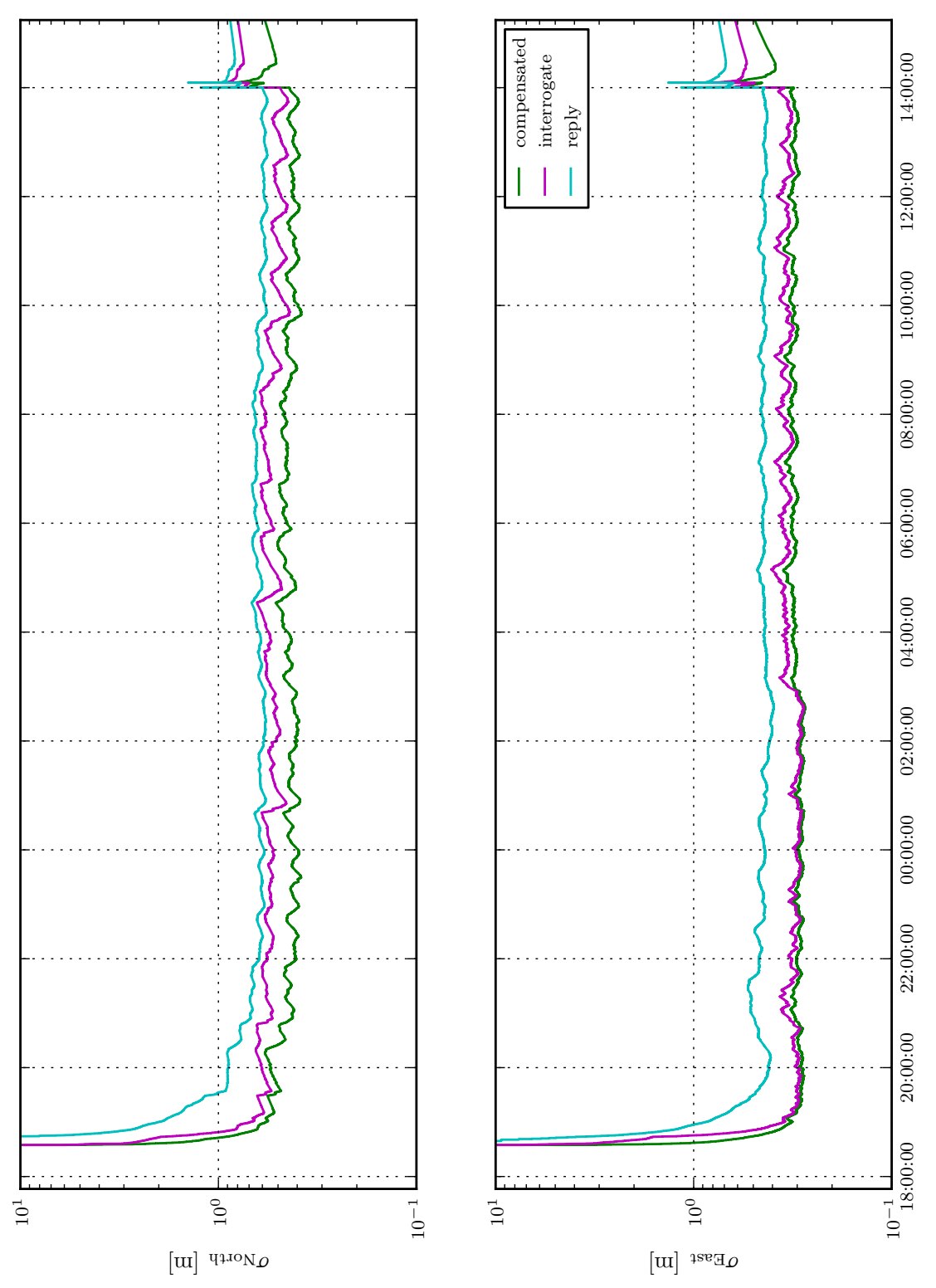

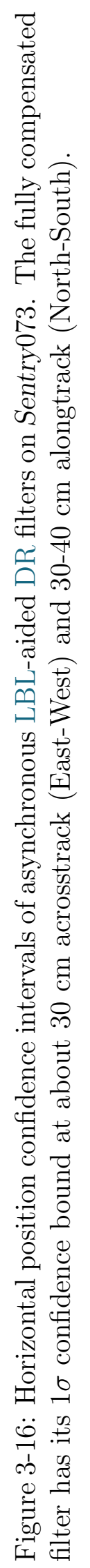


measurement fusion based on the workhorse KF framework, future directions to investigate might include:

- principled delay treatment in internal estimates of the shipboard USBL system

- other operations concepts and associated delay structures (e.g., USBL on a tethered vehicle like an ROV)

- more complicated acoustic travel time models using raytracing

- incorporating measurements from bounce (return) paths of LBL without needing any additional augmented states.

- incorporating wrapped LBL replies in very long range missions by using additional augmented states

- using the asynchronous LBL example presented here, with explicit delay compensation, in a single transponder or mobile transponder navigation scenario 


\section{Chapter 4}

\section{Discussion and Conclusions}

This dissertation has presented details on three contributions to the art of automated realtime underwater navigation. The theory behind each of these contributions has been explained in detail. And each contribution has been demonstrated in simulation, with laboratory data, or in the field. Some of these contributions have been demonstrated in all three arenas.

Here we briefly review the results and conclusions from each chapter, and comment on promising future directions for each research thread.

\section{Navigation Sensor Alignment}

We presented the Doppler velocity log (DVL)/fiber-optic gyroscope (FOG) sensor alignment problem and posed it as a general rotation identification problem. After expressing the problem in Geometric Algebra (GA), we proposed a simple adaptive identifier and proved its asymptotic stability. We demonstrated this identifier in simulation, then applied it to DVL/FOG alignment on underwater vehicles in the laboratory and in the field. Using the alignment estimated by the rotor identifier in dead reckoning (DR) improved agreement of the position estimate with long baseline (LBL) observations. The rotor identifier performed comparably to previously reported techniques based on rotation matrices.

Future directions one might take with the rotor identifier include: 
- investigation of the acceleration-velocity form of DVL/inertial navigation system (INS) alignment as studied using rotation matrices in [62,63]

- a proportional-derivative form of the identifier for enhanced noise rejection and increased control of identifier dynamics

- an identifier on the full group of rigid-body motions-rotation as well as translationusing rotors in a higher dimensional GA

- applications to align other sensors (camera, multibeam, etc...)

Finally, the clarity and intuition gained by posing the rotation identification problem in GA has proven useful in this first application of GA to underwater robotics. It is possible that similar benefits are waiting if we apply GA to other underwater problems (e.g., hydrodynamics, the full six degree-of-freedom navigation equations, or control problems)

\section{Dead reckoning through the water column}

We proposed a method for dead reckoning through the water column using overlapping water profile measurements collected by a vehicle-mounted acoustic Doppler current profiler (ADCP). We demonstrated this proof of concept using three implementations:

- an online bin average approach,

- a batch weighted least squares (WLS) approach, and

- an online recursive least squares (RLS) approach.

We applied these implementations to data from the ascent at the end of several Sentry missions, and compared the DR track to external ultra-short baseline (USBL) observations where possible.

Although this technique is not quite ready for field service on deep autonomous underwater vehicles (AUVs), it holds great promise. Dead reckoning using ADCP water profile measurements enhances the autonomy of underwater vehicles because it 
enables underwater navigation without dependence on external systems. It provides a link between Global Positioning System (GPS) at the surface and DR or terrainrelative navigation near the seafloor.

Future work in this thread should focus on demonstrating a full operation-from surface to seafloor and back-on a small AUV, possibly with higher frequency ADCP.

\section{Model-driven delayed measurement fusion}

We've presented a flexible, model-driven approach to fuse information from delayed measurements in realtime. We used the proven framework of Kalman filter, and our model-driven approach does not change any of the underlying machinery of the Kalman filter (KF). We demonstrated the approach in simulation on a damped harmonic oscillator (DHO), and showed that the filter with explicit delay compensation approached the performance of a filter without any measurement delay. We replicated this result using real-world data from Sentry for USBL-aided DR, then applied it to asynchronous LBL-aided DR, again using field data from Sentry.

With a straightforward approach to delayed measurement fusion based on the workhorse KF framework, future directions to investigate might include:

- rigorous delay treatment in internal estimates of the shipboard USBL system

- other operations concepts and associated delay structures (e.g., USBL on a tethered vehicle like an remotely operated vehicle (ROV))

- more complicated acoustic travel time models using raytracing

- incorporating measurements from bounce (return) paths of LBL without needing any additional augmented states.

- incorporating wrapped LBL replies in very long range missions by using additional augmented states

- using the asynchronous LBL example presented here, with explicit delay compensation, in a single transponder or mobile transponder navigation scenario 
The sigma point Kalman filter (SPKF) foundation of the USBL-aided DR and LBLaided DR field examples is ideally posed to leverage high-fidelity dynamic vehicle models. Including these (instead of a simple kinematic model) will make each filter implementation vehicle- or application specific, but promises the added benefit of robustness to sensor outages and potentially increased capability for fault detection.

In the end, I find the more I know, the less I know. This dissertation has raised more questions than it has answered, but it has answered the ones it set out to. Underwater navigation remains an interesting and challenging field, one simply has to choose the next topic and dive in. 


\section{Bibliography}

[1] J. Buffett, Where is Joe Merchant? : A Novel Tale. Harcourt Brace Jovanovich, August 1992.

[2] N. P. Fofonoff and R. C. Millard Jr., "Algorithms for computation of fundamental properties of seawater," in UNESCO Technical Papers in Marine Science. Place de Fontenoy, 75700 Paris, France: UNESCO Division of Marine Sciences, 1983, no. 44.

[3] M. M. Hunt, W. M. Marquet, D. A. Moller, K. R. Peal, W. K. Smith, and R. C. Spindel, "An acoustic navigation system," Woods Hole Oceanographic Institution, Woods Hole, MA, Technical Report 74-6, December 1974, unpublished manuscript.

[4] P. H. Milne, Underwater Acoustic Positioning Systems. Gulf Publishing Company, 1983.

[5] M. V. Jakuba, C. N. Roman, H. Singh, C. Murphy, C. Kunz, C. Willis, T. Sato, and R. A. Sohn, "Long-baseline acoustic navigation for under-ice autonomous underwater vehicle operations," Journal of Field Robotics, vol. 25, no. 11-12, pp. 861 - 879, 2008. [Online]. Available: http://dx.doi.org/10.1002/rob.20250

[6] L. L. Whitcomb, D. R. Yoerger, H. Singh, and J. C. Howland, "Advances in underwater robot vehicles for deep ocean exploration: Navigation, control, and survey operations," in Proceedings of the International Symposium of Robotics Research (ISRR), Snowbird, UT, October 1999.

[7] J. C. Kinsey, R. M. Eustice, and L. L. Whitcomb, "A survey of underwater vehicle navigation: Recent advances and new challenges," in IFAC Conference of Manoeuvering and Control of Marine Craft, Lisbon, Portugal, September 2006, invited paper.

[8] M. Larsen, "Synthetic long baseline navigation of underwater vehicles," in Proceedings of OCEANS, vol. 3. MTS/IEEE, 2000, pp. 2043-2050.

[9] C. E. G. LaPointe, "Virtual long baseline (VLBL) autonomous underwater vehicle navigation using a single transponder," Master's thesis, Massachusetts Institute of Technology and Woods Hole Oceanographic Institution, Cambridge and Woods Hole, MA, June 2006. 
[10] B. Jalving, K. Gade, O. Hagen, and K. Vestgard, "A toolbox of aiding techniques for the HUGIN AUV integrated inertial navigation system," in Proceedings of OCEANS, vol. 2. MTS/IEEE, September 2003, pp. 1146-1153.

[11] O. Hegrenaes, K. Gade, O. Hagen, and P. Hagen, "Underwater transponder positioning and navigation of autonomous underwater vehicles," in Proceedings of OCEANS. MTS/IEEE, October 2009.

[12] J.-P. Peyronnet, R. Person, and F. Rybicki, "POSIDONIA 6000: a new long range highly accurate ultra short base line positioning system," in Proceedings of OCEANS, vol. 3. Nice, France: MTS/IEEE, September 1998, pp. 1721 -1727. [Online]. Available: http://dx.doi.org/10.1109/OCEANS.1998.726382

[13] J. C. Kinsey and L. L. Whitcomb, "In situ alignment calibration of attitude and Doppler sensors for precision underwater vehicle navigation: Theory and experiment," IEEE Journal of Oceanic Engineering, vol. 32, no. 2, pp. 286-299, April 2007. [Online]. Available: http://dx.doi.org/10.1109/JOE.2007.893686

[14] — - "Adaptive identification on the group of rigid-body rotations and its application to underwater vehicle navigation," IEEE Transactions on Robotics, vol. 23, no. 1, pp. 124-136, February 2007. [Online]. Available: http://dx.doi.org/10.1109/TRO.2006.886829

[15] M. J. Stanway and J. C. Kinsey, "Sensor alignment using rotors in geometric algebra," in Proceedings of the International Conference on Robotics and Automation (ICRA). Shanghai, China: IEEE, May 2011, pp. 994-999. [Online]. Available: http://dx.doi.org/10.1109/ICRA.2011.5980519

[16] W. Alameda Jr, "Seadevil-A totally integrated inertial navigation system (INS) solution," in Proceedings of the Underwater Intervention Symposium, New Orleans, LA, 2002.

[17] Navigator Doppler Velocity Log Technical Manual, Teledyne RD Instruments, Poway, CA, November 2006.

[18] N. Brokloff, "Matrix algorithm for Doppler sonar navigation," in Proceedings of OCEANS, vol. 3. MTS/IEEE, September 1994, pp. 378-383. [Online]. Available: http://dx.doi.org/10.1109/OCEANS.1994.364228

[19] L. Whitcomb, D. Yoerger, and H. Singh, "Advances in doppler-based navigation of underwater robotic vehicles," in Proceedings of the International Conference on Robotics and Automation (ICRA), vol. 1. IEEE, May 1999, pp. 399-406. [Online]. Available: http://dx.doi.org/10.1109/ROBOT.1999.770011

[20] L. Whitcomb, D. Yoerger, H. Singh, and D. Mindell, "Towards precision robotic maneuvering, survey, and manipulation in unstructured undersea environments," in Proceedings of the International Symposium on Robotics Research (ISRR), vol. 8, 1998, pp. 45-54. 
[21] J. C. Kinsey and L. L. Whitcomb, "Preliminary field experience with the DVLNAV integrated navigation system for oceanographic submersibles," Control Engineering Practice, vol. 12, no. 12, pp. 1541-1548, December 2004.

[22] P. Miller, J. Farrell, Y. Zhao, and V. Djapic, "Autonomous underwater vehicle navigation," IEEE Journal of Oceanic Engineering, vol. 35, no. 3, pp. 663 -678, July 2010. [Online]. Available: http://dx.doi.org/10.1109/JOE.2010.2052691

[23] R. McEwen, H. Thomas, D. Weber, and F. Psota, "Performance of an AUV navigation system at Arctic latitudes," IEEE Journal of Oceanic Engineering, vol. 30, no. 2, pp. 443-454, April 2005. [Online]. Available: http://dx.doi.org/10.1109/JOE.2004.838336

[24] N. Brokloff, "Dead reckoning with an ADCP and current extrapolation," in Proceedings of OCEANS, vol. 2. MTS/IEEE, October 1997, p. 1411. [Online]. Available: http://dx.doi.org/10.1109/OCEANS.1997.624203

[25] E. Firing and R. Gordon, "Deep ocean acoustic doppler current profiling," in Proceedings of the IEEE Fourth Working Conference on Current Measurement, April 1990, pp. 192 -201. [Online]. Available: http://dx.doi.org/10.1109/CURM.1990.110905

[26] J. Fischer and M. Visbeck, "Deep velocity profiling with selfcontained ADCPs," Journal of Atmospheric and Oceanic Technology, vol. 10, no. 5, pp. 764-773, 1993. [Online]. Available: http://dx.doi.org/10.1175/1520-0426(1993)010\%3C0764:DVPWSC\%3E2.0.CO;2

[27] M. Visbeck, "Deep velocity profiling using lowered acoustic Doppler current profilers: Bottom track and inverse solutions," Journal of Atmospheric and Oceanic Technology, vol. 19, no. 5, pp. 794-807, 2002. [Online]. Available: http://dx.doi.org/10.1175/1520-0426(2002)019\%3C0794:DVPULA\%3E2.0.CO;2

[28] M. J. Stanway, "Water profile navigation with an acoustic Doppler current profiler," in Proceedings of OCEANS. Sydney, Australia: MTS/IEEE, May 2010. [Online]. Available: http://dx.doi.org/10.1109/OCEANSSYD.2010.5603647

[29] — - "Dead reckoning through the water column with an acoustic Doppler current profiler: Field experiences," in Proceedings of OCEANS. Kona, Hawai'i: IEEE, September 2011.

[30] L. Medagoda, M. Jakuba, O. Pizarro, and S. Williams, "Water column current profile aided localisation for autonomous underwater vehicles," in Proceedings of OCEANS. Sydney, Australia: IEEE, May 2010.

[31] L. Medagoda, S. B. Williams, O. Pizarro, and M. V. Jakuba, "Water column current profile aided localisation combined with view-based SLAM for autonomous underwater vehicle navigation," in Proceedings of the International Conference on Robotics and Automation (ICRA). Shanghai, China: IEEE, May 2011. 
[32] D. Meduna, S. Rock, and R. McEwen, "Low-cost terrain relative navigation for long-range AUVs," in Proceedings of OCEANS. MTS/IEEE, September 2008. [Online]. Available: http://dx.doi.org/10.1109/OCEANS.2008.5152043

[33] — - "Closed-loop terrain relative navigation for AUVs with noninertial grade navigation sensors," in Proceedings of Autonomous Underwater Vehicles $(A U V)$. IEEE/OES, September 2010. [Online]. Available: http://dx.doi.org/10.1109/AUV.2010.5779659

[34] D. di Massa and W. J. Stewart, "Terrain-relative navigation for autonomous underwater vehicles," in Proceedings of OCEANS, vol. 1. MTS/IEEE, October 1997, pp. 541-546.

[35] J. Moryl, H. Rice, and S. Shinners, "The universal gravity module for enhanced submarine navigation," in Proceedings of Position Location and Navigation Symposium. IEEE, April 1998, pp. 324-331.

[36] K. Åndnonsen and O. Hagen, "An analysis of real-time terrain aided navigation results from a HUGIN AUV," in Proceedings of OCEANS, September 2010. [Online]. Available: http://dx.doi.org/10.1109/OCEANS.2010.5664076

[37] P. Newman, J. Leonard, and R. Rikoski, "Towards constant-time SLAM on an autonomous underwater vehicle using synthetic aperture sonar," in Proceedings of the International Symposium of Robotics Research (ISRR). Springer, 2003.

[38] C. Roman and H. Singh, "Improved vehicle based multibeam bathymetry using sub-maps and slam," in Proceedings of the International Conference on Intelligent Robots and Systems (IROS). IEEE/RSJ, August 2005, pp. 3662-3669. [Online]. Available: http://dx.doi.org/10.1109/IROS.2005.1545340

[39] S. Barkby, S. Williams, O. Pizarro, and M. Jakuba, "An efficient approach to bathymetric slam," in Proceedings of the International Conference on Intelligent Robots and Systems (IROS). IEEE/RSJ, October 2009, pp. 219-224. [Online]. Available: http://dx.doi.org/10.1109/IROS.2009.5354248

[40] P. Newman and H. Durrant-Whyte, "Using sonar in terrain-aided underwater navigation," in Proceedings of the International Conference on Robotics and Automation (ICRA), vol. 1, May 1998, pp. 440-445. [Online]. Available: http://dx.doi.org/10.1109/ROBOT.1998.677013

[41] C. Roman, "Self consistent bathymetric mapping from robotic vehicles in the deep ocean," Ph.D. dissertation, Massachusetts Institute of Technology and Woods Hole Oceanographic Institution, Cambridge and Woods Hole, MA, 2005.

[42] R. M. Eustice, "Large-area visually augmented navigation for autonomous underwater vehicles," Ph.D. dissertation, Massachusetts Institute of Technology and Woods Hole Oceanographic Institution, Cambridge and Woods Hole, MA, 2005. 
[43] R. Eustice, H. Singh, and J. Leonard, "Exactly sparse delayed-state filters," in Proceedings of the International Conference on Robotics and Automation (ICRA), April 2005, pp. 2417-2424. [Online]. Available: http://dx.doi.org/10.1109/ROBOT.2005.1570475

[44] R. M. Eustice, H. Singh, and J. J. Leonard, "Exactly sparse delayedstate filters for view-based SLAM," IEEE Transactions on Robotics, vol. 22, no. 6, pp. 1100-1114, December 2006. [Online]. Available: http://dx.doi.org/10.1109/TRO.2006.886264

[45] R. Eustice, O. Pizarro, and H. Singh, "Visually augmented navigation for autonomous underwater vehicles," IEEE Journal of Oceanic Engineering, vol. 33, no. 2, pp. 103-122, April 2008. [Online]. Available: http://dx.doi.org/10.1109/JOE.2008.923547

[46] P. Kimball and S. Rock, "Sonar-based iceberg-relative AUV navigation," in Proceedings of Autonomous Underwater Vehicles (AUV). IEEE/OES, October 2008. [Online]. Available: http://dx.doi.org/10.1109/AUV.2008.5290534

[47] _ - "Estimation of iceberg motion for mapping by auvs," in Proceedings of Autonomous Underwater Vehicles (AUV). IEEE/OES, September 2010. [Online]. Available: http://dx.doi.org/10.1109/AUV.2010.5779664

[48] D. R. Yoerger, A. M. Bradley, M. V. Jakuba, M. A. Tivey, C. R. German, T. M. Shank, and R. W. Embley, "Mid-Ocean Ridge Exploration with an Autonomous Underwater Vehicle," Oceanography, vol. 20, no. 4, p. 52, 2007.

[49] M. V. Jakuba and D. R. Yoerger, "High-resolution multibeam sonar mapping with the autonomous benthic explorer (ABE)," in Proceedings of the 13th Unmanned Untethered Submersible Technology Conference, Durham, NH, 2003.

[50] C. R. German, D. R. Yoerger, M. Jakuba, T. M. Shank, C. H. Langmuir, and K.-i. Nakamura, "Hydrothermal exploration with the Autonomous Benthic Explorer," Deep Sea Research Part I: Oceanographic Research Papers, vol. 55, no. 2, pp. 203 - 219, 2008. [Online]. Available: http://dx.doi.org/10.1016/j.dsr.2007.11.004

[51] R. Camilli and A. Duryea, "Characterizing marine hydrocarbons with in-situ mass spectrometry," in Proceedings of OCEANS. MTS/IEEE, October 2007. [Online]. Available: http://dx.doi.org/10.1109/OCEANS.2007.4449412

[52] D. L. Valentine, C. M. Reddy, C. Farwell, T. M. Hill, O. Pizarro, D. R. Yoerger, R. Camilli, R. K. Nelson, E. E. Peacock, and S. C. Bagby, "Asphalt volcanoes as a potential source of methane to late Pleistocene coastal waters," Nature Geoscience, 2010, in press.

[53] R. Camilli, C. M. Reddy, D. R. Yoerger, B. A. S. Van Mooy, M. V. Jakuba, J. C. Kinsey, C. P. McIntyre, S. P. Sylva, and J. V. Maloney, 
"Tracking hydrocarbon plume transport and biodegradation at deepwater horizon," Science, vol. 330, no. 6001, pp. 201-204, 2010. [Online]. Available: http://www.sciencemag.org/content/330/6001/201.abstract

[54] M. J. Stanway, "Delayed-state sigma point Kalman filters for underwater navigation," in Proceedings of Autonomous Underwater Vehicles $(A U V)$. Monterey, CA: IEEE/OES, September 2010. [Online]. Available: http://dx.doi.org/10.1109/AUV.2010.5779652

[55] J. C. Kinsey and L. L. Whitcomb, "Towards in-situ calibration of gyro and doppler navigation sensors for precision underwater vehicle navigation," in Proceedings of the International Conference on Robotics and Automation (ICRA), vol. 4. IEEE, 2002, pp. 4016-4023. [Online]. Available: http://dx.doi.org/10.1109/ROBOT.2002.1014364

[56] G. Strang, Introduction to Applied Mathematics. Wellesley, MA: WellesleyCambridge Press, 1986.

[57] K. S. Arun, T. S. Huang, and S. D. Blostein, "Least-squares fitting of two 3-D point sets," IEEE Transactions on Pattern Analysis and Machine Intelligence (PAMI), vol. 9, no. 5, pp. 698-700, September 1987. [Online]. Available: http://dx.doi.org/10.1109/TPAMI.1987.4767965

[58] S. Umeyama, "Least-squares estimation of transformation parameters between two point patterns," IEEE Transactions on Pattern Analysis and Machine Intelligence (PAMI), vol. 13, no. 5, pp. 376-380, 1991. [Online]. Available: http://dx.doi.org/10.1109/34.88573

[59] C. Doran, D. Hestenes, F. Sommen, and N. van Acker, "Lie groups as spin groups," Journal of Mathematical Physics, vol. 34, no. 8, pp. 3642-3669, August 1993.

[60] F. Bullo and R. M. Murray, "Proportional derivative (PD) control on the Euclidean group," California Institute of Technology, Technical Report Caltech/CDS 95-010, May 1995, short version appeared in the Proc. of ECC'95. [Online]. Available: http://resolver.caltech.edu/CaltechCDSTR:1995.CIT-CDS-95-010

[61] J. C. Kinsey and L. L. Whitcomb, "Adaptive identification on the group of rigid body rotations," in Proceedings of the International Conference on Robotics and Automation (ICRA). IEEE, April 2005, pp. 3256-3261.

[62] G. Troni and L. Whitcomb, "New methods for in-situ calibration of attitude and doppler sensors for underwater vehicle navigation: Preliminary results," in Proceedings of OCEANS. MTS/IEEE, September 2010. [Online]. Available: http://dx.doi.org/10.1109/OCEANS.2010.5664279 
[63] — , "Experimental evaluation of new methods for in-situ calibration of attitude and doppler sensors for underwater vehicle navigation," in Proceedings of the International Conference on Intelligent Robots and Systems (IROS). IEEE/RSJ, 2011. [Online]. Available: http://dx.doi.org/10.1109/IROS.2011.6048629

[64] C. J. L. Doran, "Bayesian inference and geometric algebra: an application to camera localization," in Geometric algebra: a geometric approach to computer vision, neural and quantum computing, robotics and engineering., E. BayroCorrochano and G. Sobczyk, Eds. Birkhauser, 2000.

[65] S. Buchholz and G. Sommer, "On averaging in Clifford groups," Computer Algebra and Geometric Algebra with Applications, pp. 229-238, 2005. [Online]. Available: http://dx.doi.org/10.1007/11499251_19

[66] F. Bullo, R. M. Murray, and A. Sarti, "Control on the sphere and reduced attitude stabilization," California Institute of Technology, Technical Report Caltech/CDS 95-005, Jan. 1995, short version appeared in Proc. of the 1995 IFAC Symposium on Nonlinear control systems design (NOLCOS). [Online]. Available: http://resolver.caltech.edu/CaltechCDSTR:1995.CIT-CDS-95-005

[67] D. Hestenes, New Foundations for Classical Mechanics, 2nd ed., ser. Fundamental Theories of Physics. Springer Netherlands, 2002, vol. 99.

[68] C. Doran and A. Lasenby, Geometric Algebra for Physicists. Cambridge, UK; New York, NY: Cambridge University Press, 2003.

[69] L. Dorst, D. Fontijne, and S. Mann, Geometric algebra for computer science: an object-oriented approach to geometry, ser. Morgan Kaufmann series in computer graphics and geometric modeling. Amsterdam: Elsevier, 2007.

[70] S. F. Gull, A. N. Lasenby, and C. J. L. Doran, "Imaginary numbers are not real - the geometric algebra of spacetime," Foundations of Physics, vol. 23, no. 9, pp. 1175-1201, 1993.

[71] E. Corrochano and G. Sobczyk, Eds., Geometric algebra with applications in science and engineering. Boston: Birkhauser, 2001.

[72] E. Bayro-Corrochano, Handbook of Geometric Computing: Applications in Pattern Recognition, Computer Vision, Neuralcomputing, and Robotics. Berlin: Springer, 2005.

[73] — Geometric Computing: for Wavelet Transforms, Robot Vision, Learning, Control and Action. London: Springer, 2010.

[74] E. Bayro-Corrochano and G. Scheuermann, Eds., Geometric Algebra Computing in Engineering and Computer Science. London: Springer, 2010. 
[75] D. Hestenes, "New tools for computational geometry andrejuvenation of screw theory," in Geometric Algebra Computing, E. Bayro-Corrochano and G. Scheuermann, Eds. Springer London, 2010, pp. 3-33. [Online]. Available: http://dx.doi.org/10.1007/978-1-84996-108-0

[76] C. M. Brown, "Some computational properties of rotation representations," University of Rochester, Computer Science Department., Rochester, New York, Tech. Rep. TR 303, August 1989. [Online]. Available: http://hdl.handle.net/1802/5540

[77] W. G. Breckenridge, "Quaternions - proposed standard conventions," Interoffice Memorandum, NASA Jet Propulsion Laboratory, Tech. Rep. IOM 343-79-1199, 1999, interoffice Memorandum.

[78] J. A. Farrell, Aided Navigation: GPS with High Rate Sensors. New York, NY, USA: McGraw-Hill, 2008.

[79] E. Cartan, The Theory of Spinors, 1st ed. Cambridge, MA: MIT Press, 1966.

[80] D. Fontijne. Gaigen2.5 Geometric Algebra implementation generator. Visited: 29 September 2011. [Online]. Available: http://g25.sourceforge.net/

[81] D. Fontijne, T. Bouma, and L. Dorst. (2001) Gaigen: a Geometric Algebra implementation generator. [Online]. Available: http://www.science.uva.nl/ga/gaigen/

[82] D. Fontijne, "Gaigen 2: a Geometric Algebra implementation generator," in Proceedings of ACM GPCE, 2006.

[83] H. K. Khalil, Nonlinear Systems, 2nd ed. New Jersey: Prentice-Hall, 1996.

[84] R. M. Murray, Z. Li, and S. S. Sastry, A Mathematical Introduction to Robotic Manipulation. Washington, D.C.: CRC Press, 1994. [Online]. Available: http://www.cds.caltech.edu/ murray/mlswiki

[85] J. C. Kinsey, "Advances in precision navigation of oceanographic submersibles," Ph.D. dissertation, Johns Hopkins University, Baltimore, MD, 2007.

[86] J. Kinsey, D. Smallwood, and L. Whitcomb, "A new hydrodynamics test facility for uuv dynamics and control research," in Proceedings of OCEANS, vol. 1. MTS/IEEE, September 2003, pp. 356-361. [Online]. Available: http://dx.doi.org/10.1109/OCEANS.2003.178587

[87] M. J. Stanway, "Contributions to automated realtime underwater navigation," in Proceedings of the 17th International Unmanned Untethered Submersible Technology Conference (UUST). Portsmouth, New Hampshire: AUSI, August 2011. 
[88] M. S. Grewal and A. P. Andrews, Kalman Filtering: Theory and Practice Using MATLAB, 2nd ed. John Wiley \& Sons, 2001.

[89] S. Haykin, Adaptive Filter Theory, 4th ed. Upper Saddle River, New Jersey: Prentice-Hall, 2002.

[90] R. Garcia, J. Batlle, X. Cufi, and J. Amat, "Positioning an underwater vehicle through image mosaicking," in Proceedings of IEEE International Conference on Robotics and Automation, vol. 3, 2001, pp. 2779 - 2784.

[91] R. E. Kalman, "A new approach to linear filtering and prediction problems," Transactions of the ASME-Journal of Basic Engineering, vol. 82, no. Series D, pp. 35-45, 1960.

[92] K. Fauske, F. Gustafsson, and Ø. Hegrenæs, "Estimation of AUV dynamics for sensor fusion," Information Fusion, 2007 10th International Conference on, pp. 1-6, July 2007. [Online]. Available: http://dx.doi.org/10.1109/ICIF.2007.4408044

[93] S. J. Julier and J. K. Uhlmann, "A general method for approximating nonlinear transformations of probability distributions," Dept. of Engineering Science, University of Oxford, Technical Report, 1996.

[94] S. J. Julier, J. Uhlmann, and H. Durrant-Whyte, "A new method for the nonlinear transformation of means and covariances in filters and estimators," IEEE Transactions on Automatic Control, vol. 45, no. 3, pp. 477-482, Mar 2000.

[95] S. J. Julier, "The scaled unscented transformation," in Proceedings of the American Control Conference, vol. 6, 2002, pp. 4555-4559 vol.6.

[96] S. J. Julier and J. K. Uhlmann, "A new extension of the Kalman filter to nonlinear systems," in Int. Symp. Aerospace/Defense Sensing, Simul, and Controls, 1997.

[97] — - "Unscented filtering and nonlinear estimation," Proceedings of the IEEE, vol. 92, no. 3, pp. 401-422, Mar 2004.

[98] E. A. Wan and R. van der Merwe, "The unscented Kalman filter for nonlinear estimation," in Adaptive Systems for Signal Processing, Communications, and Control Symposium. AS-SPCC. IEEE, 2000, pp. 153-158.

[99] R. van der Merwe, "Sigma-point Kalman filters for probabilistic inference in dynamic state-space models," Ph.D. dissertation, Oregon Health \& Science University, Beaverton, OR, April 2004.

[100] R. van der Merwe, E. A. Wan, and S. J. Julier, "Sigma-point Kalman filters for nonlinear estimation and sensor-fusion: Applications to integrated navigation," in Proceedings of the AIAA Guidance, Navigation $\&$ Control Conference. AIAA, 2004, pp. 16-19. 
[101] R. van der Merwe and E. A. Wan, "Sigma-point Kalman filters for integrated navigation," in Proceedings of the 60th Annual Meeting of the Institute of Navigation (ION), June 2004, pp. $641-654$.

[102] S. Challa, R. J. Evans, and X. Wang, "A bayesian solution and its approximations to out-of-sequence measurement problems," Information Fusion, vol. 4, no. 3, pp. 185-199, 2003. [Online]. Available: http://dx.doi.org/10.1016/S1566-2535(03)00037-X

[103] J. Leonard and R. Rikoski, "Incorporation of delayed decision making into stochastic mapping," in Experimental Robotics VII, ser. Lecture Notes in Control and Information Sciences, D. Rus and S. Singh, Eds. Springer Berlin / Heidelberg, 2001, vol. 271, pp. 533-542. [Online]. Available: http://dx.doi.org/10.1007/3-540-45118-8_53

[104] R. J. Rikoski, "Dynamic sonar perception," Ph.D. dissertation, Massachusetts Institute of Technology, Cambridge, MA, June 2003. [Online]. Available: http://hdl.handle.net/1721.1/35702

[105] P. Ridao, D. Ribas, E. Hernandez, and A. Rusu, "USBL/DVL navigation through delayed position fixes," in Proceedings of the International Conference on Robotics and Automation (ICRA), may 2011, pp. 2344-2349. [Online]. Available: http://dx.doi.org/10.1109/ICRA.2011.5980110

[106] R. D. Hilton, D. A. Martin, and W. D. Blair, "Tracking with time-delayed data in multisensor systems," Naval Surface Warfare Center-Dahlgren Division, Dahlgren, Virginia, Tech. Rep. NSWCDD/TR-93/351, August 1993.

[107] H. L. Alexander, "State estimation for distributed systems with sensing delay," V. Libby, Ed., vol. 1470, no. 1. SPIE, 1991, pp. 103-111. [Online]. Available: http://dx.doi.org/10.1117/12.44843

[108] T. Larsen, N. Andersen, O. Ravn, and N. Poulsen, "Incorporation of time delayed measurements in a discrete-time Kalman filter," in Proceedings of the 37th IEEE Conference on Decision \& Control, vol. 4, Tampa, FL, December 1998, pp. 3972-3977.

[109] M. Bak, T. Larsen, M. Norgaard, N. Andersen, N. Poulsen, and O. Ravn, "Location estimation using delayed measurements," in 5th International Workshop on Advanced Motion Control (AMC). IEEE, June 1998, pp. 180-185. [Online]. Available: http://dx.doi.org/10.1109/AMC.1998.743533

[110] "Nomenclature for treating the motion of a submerged body through a fluid," SNAME, Technical and Research Bulletin 1-5, 1950.

[111] T. I. Fossen, Guidance and Contol of Ocean Vehicles. New York: John Wiley \& Sons, 1994. 
[112] D. R. Yoerger, M. Jakuba, A. M. Bradley, and B. Bingham, "Techniques for deep sea near bottom survey using an autonomous underwater vehicle," The International Journal of Robotics Research, vol. 26, no. 1, pp. 41-54, 2007. [Online]. Available: http://dx.doi.org/10.1177/0278364907073773

[113] C. E. G. LaPointe, "A parallel hypothesis method of autonomous underwater vehicle navigation," Ph.D. dissertation, Massachusetts Institute of Technology and Woods Hole Oceanographic Institution, Cambridge and Woods Hole, MA, June 2009.

[114] E. Olson, J. J. Leonard, and S. Teller, "Robust range-only beacon localization," Oceanic Engineering, IEEE Journal of, vol. 31, no. 4, pp. 949 -958, 2006. 


\section{Acronyms}

AUV autonomous underwater vehicle

ROV remotely operated vehicle

HOV human occupied vehicle

ABE autonomous benthic explorer

UAV unmanned aerial vehicle

UGV unmanned ground vehicle

DR dead reckoning

SLAM simultaneous localization and mapping

LBL long baseline (acoustic positioning system)

SBL short baseline (acoustic positioning system)

USBL ultra-short baseline (acoustic positioning system)

SLBL synthetic long baseline (acoustic positioning system)

VLBL virtual long baseline (acoustic positioning system)

UTP underwater transponder positioning

TRN terrain relative navigation

VAN visually aided navigation

GPS Global Positioning System

ADCP acoustic Doppler current profiler

DVL Doppler velocity log

LADCP lowered acoustic Doppler current profiler

INS inertial navigation system

IMU inertial measurement unit

FOG fiber-optic gyroscope

RLG ring-laser gyroscope

AHRS attitude and heading reference system

LADCP Lowered Acoustic Doppler Current Profiler 


\begin{tabular}{|c|c|}
\hline GA & Geometric Algebra \\
\hline LA & Linear Algebra \\
\hline SVD & singular value decomposition \\
\hline MMSE & minimum mean-squared error \\
\hline LS & least squares \\
\hline WLS & weighted least squares \\
\hline RLS & recursive least squares \\
\hline CLS & constrained least squares \\
\hline WSLR & weighted statistical linear regression \\
\hline KF & Kalman filter \\
\hline ESFR & error-state feedback regulator \\
\hline EKF & extended Kalman filter \\
\hline UT & unscented transform \\
\hline SUT & scaled unscented transform \\
\hline UKF & unscented Kalman filter \\
\hline SPKF & sigma point Kalman filter \\
\hline DS-KF & delayed-state Kalman filter \\
\hline DS-SPKF & delayed-state sigma point Kalman filter \\
\hline IF & information filter \\
\hline DSSM & discrete state space model \\
\hline HMM & hidden Markov model \\
\hline JHU & Johns Hopkins University \\
\hline DHO & damped harmonic oscillator \\
\hline
\end{tabular}




\section{Appendix A}

\section{Sigma Point Kalman Filters}

This appendix is included to give the reader a basic introduction to the unscented Kalman filter (UKF), which was used as the underlying framework for USBL-aided dead reckoning in section 3.5 and LBL-aided dead reckoning in section 3.6.

\section{A.1 The Unscented Transform}

Julier \& Uhlmann introduced the scaled unscented transform as a general method for approximating nonlinear transformations of random variable distributions in [93,95]. It is a derivative-free method, in that it requires no analytical Jacobians, only a forward model of the transformation. It performs weighted statistical linear regression (WSLR) on a small set of deterministically selected sample points to approximate the moments of the transformed random variable distribution.

Consider a random variable $\boldsymbol{x} \in \mathbb{R}^{p}$. The scaled unscented transform parameters $\alpha, \beta, \kappa$, define the sigma point scaling factor:

$$
\varsigma=\alpha \sqrt{p+\kappa}
$$

and the sigma point weights:

$$
\gamma=\varsigma^{2}=\alpha^{2}(p+\kappa)
$$




$$
\begin{aligned}
\mathrm{w}_{0}^{m} & =\frac{\gamma-p}{\gamma} \\
\mathrm{w}_{0}^{c} & =\mathrm{w}_{0}^{m}+1-\alpha^{2}+\beta \\
\mathrm{w}_{i}^{m}=\mathrm{w}_{i}^{c} & =\frac{1}{2 \gamma} \quad i=1, \ldots, 2 p+1
\end{aligned}
$$

Refer to $[95,99]$ for guidance on selecting appropriate parameters.

The scaled unscented transform (SUT) begins by deterministically sampling the original random variable. This generates $2 p+1$ sigma points:

$$
\mathcal{X}=\left[\begin{array}{lll}
\overline{\boldsymbol{x}} & \overline{\boldsymbol{x}}+\varsigma \sqrt{\mathbf{P}_{\boldsymbol{x}}} & \overline{\boldsymbol{x}}-\varsigma \sqrt{\mathbf{P}_{\boldsymbol{x}}}
\end{array}\right]
$$

where $\overline{\boldsymbol{x}}$ denotes the expected value of the random variable $\boldsymbol{x}$, and $\sqrt{\mathbf{P}_{\boldsymbol{x}}}$ is the matrix square root of the covariance (e.g. by Cholesky decomposition).

The nonlinear function $g(\boldsymbol{x}, \boldsymbol{u})$ then transforms these sigma points:

$$
\mathcal{Y}=g(\mathcal{X}, \boldsymbol{u})
$$

and the first two characteristic moments of the transformed random variable are approximated by the weighted sample mean and covariance of the transformed sigma points :

$$
\begin{aligned}
\overline{\boldsymbol{y}} & \simeq \sum_{i=0}^{2 p} \mathrm{w}_{i}^{m} \mathcal{Y}_{i} \\
\mathbf{P}_{\tilde{\boldsymbol{y}} \tilde{\boldsymbol{y}}} & \simeq \sum_{i=0}^{2 p} \mathrm{w}_{i}^{c}\left(\mathcal{Y}_{i}-\overline{\boldsymbol{y}}\right)\left(\mathcal{Y}_{i}-\overline{\boldsymbol{y}}\right)^{T}
\end{aligned}
$$

where $\mathcal{Y}_{i}$ denotes the $i$-th column of the transformed sigma point matrix.

\section{A.2 The Unscented Kalman Filter}

The predict step of the unscented Kalman filter (UKF) is simply a recursive application of the SUT to propagate the state vector $\boldsymbol{x}_{k}$ through process and measurement 
models. Equation (3.7a) then gives the Kalman gain, and the a priori predictions are updated using the measurement when it is available.

The UKF handles general process and measurement noise through state augmentation $[93,98,99]$. This ensures that uncertainty in noise is treated in the same manner as uncertainty in state.

In this study, we augment the state first with the delayed measurements, then with the noise variables:

$$
\boldsymbol{x}_{k}^{a}=\left[\begin{array}{c}
\boldsymbol{x}_{k} \\
\boldsymbol{y}_{k+n}^{d} \\
\boldsymbol{v}_{k} \\
\boldsymbol{w}_{k}
\end{array}\right]=\left[\begin{array}{c}
\boldsymbol{x}^{s} \\
\boldsymbol{x}^{v} \\
\boldsymbol{x}^{w}
\end{array}\right]_{k}
$$

The state prediction step is:

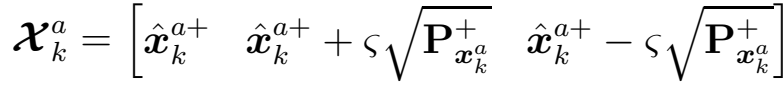

$$
\begin{aligned}
& \mathcal{X}_{k+1}^{s-}=f\left(\mathcal{X}_{k}^{s}, \boldsymbol{u}_{k}, \mathcal{X}_{k}^{v}\right) \\
& \hat{\boldsymbol{x}}_{k+1}^{s-}=\sum_{i=0}^{2 p} \mathrm{w}_{i}^{m} \mathcal{X}_{i, k+1}^{s-} \\
& \mathbf{P}_{\tilde{\boldsymbol{x}}_{k+1}^{s}}^{-}=\sum_{i=0}^{2 p} \mathrm{w}_{i}^{c}\left(\mathcal{X}_{i, k+1}^{s-}-\hat{\boldsymbol{x}}_{k+1}^{s-}\right)\left(\mathcal{X}_{i, k+1}^{s-}-\hat{\boldsymbol{x}}_{k+1}^{s-}\right)^{T}
\end{aligned}
$$

the measurement prediction and calculation of the Kalman gain can be done before the measurement is actually received:

$$
\begin{aligned}
\mathcal{Y}_{k+1}^{-} & =h\left(\boldsymbol{\mathcal { X }}_{k+1}^{s-}, \boldsymbol{u}_{k}, \boldsymbol{\mathcal { X }}_{k+1}^{w}\right) \\
\hat{\boldsymbol{y}}_{k+1}^{-} & =\sum_{i=0}^{2 p} \mathrm{w}_{i}^{m} \mathcal{Y}_{i, k+1}^{-} \\
\mathbf{P}_{\tilde{\boldsymbol{y}}_{k+1}}^{-} & =\sum_{i=0}^{2 p} \mathrm{w}_{i}^{c}\left(\mathcal{Y}_{i, k+1}^{-}-\hat{\boldsymbol{y}}_{k+1}^{-}\right)\left(\mathcal{Y}_{i, k+1}^{-}-\hat{\boldsymbol{y}}_{k+1}^{-}\right)^{T} \\
\mathbf{P}_{\tilde{\boldsymbol{x}}_{k+1}^{s}}^{-} \tilde{\boldsymbol{y}}_{k+1} & =\sum_{i=0}^{2 p} \mathrm{w}_{i}^{c}\left(\mathcal{X}_{i, k+1}^{s-}-\hat{\boldsymbol{x}}_{k+1}^{s-}\right)\left(\mathcal{Y}_{i, k+1}^{-}-\hat{\boldsymbol{y}}_{k+1}^{-}\right)^{T} \\
\mathbf{K}_{k+1} & =\mathbf{P}_{\tilde{\boldsymbol{x}}_{k+1}^{s}}^{-} \tilde{\boldsymbol{y}}_{k+1}\left(\mathbf{P}_{\tilde{\boldsymbol{y}}_{k+1}}^{-}\right)^{-1}
\end{aligned}
$$


Now the filter is ready to update the a priori estimates as soon as it receives the measurement:

$$
\begin{aligned}
& \hat{\boldsymbol{x}}_{k}^{s+}=\hat{\boldsymbol{x}}_{k}^{s-}+\mathbf{K}_{k}\left(\boldsymbol{y}_{k}-\hat{\boldsymbol{y}}_{k}^{-}\right) \\
& \mathbf{P}_{\tilde{\boldsymbol{x}}_{k}^{s}}^{+}=\mathbf{P}_{\tilde{\boldsymbol{x}}_{k}^{s}}^{-}-\mathbf{K}_{k} \mathbf{P}_{\tilde{\boldsymbol{y}}_{k}}^{-} \mathbf{K}_{k}^{T}=\mathbf{P}_{\tilde{\boldsymbol{x}}_{k}^{s}}^{-}-\mathbf{P}_{\tilde{\boldsymbol{x}}_{k}^{s} \tilde{\boldsymbol{y}}_{k}}^{-} \mathbf{K}_{k}^{T}
\end{aligned}
$$

This is the cycle of the UKF: sample, predict, update, repeat.

Note that the noise states and covariances are not modified by this algorithm. The sigma points they generate are important for accurate and robust prediction, but not directly used during the update step. 